\title{
Development of Multifarious Cueing Systems for Cost-effective Flight Simulation Training Devices
}

by

\section{Hooman Rajaee}

\author{
A thesis submitted to \\ the Faculty of Graduate and Postdoctoral Affairs \\ in partial fulfilment of \\ the requirements for the degree of \\ Master of Applied Science \\ in
}

Aerospace Engineering

Ottawa-Carleton Institute for Mechanical and Aerospace Engineering

Department of Mechanical and Aerospace Engineering

Carleton University

Ottawa, Ontario, Canada

January 2016

Copyright (C)

2016 - Hooman Rajaee 


\section{Abstract}

This project was an investigation for the development of a high-quality cost-effective Cessna 172 type-specific flight simulation training device. The Carleton University redeveloped vector simulator (CURVS) research program consisted of multiple microprojects that were distributed to a team of researchers in the Applied Dynamics Laboratory (ADL) at Carleton University.

The main objective of the project was to develop technologies by putting together commercial-off-the-shelf components to build a flight simulation training device that is relatively inexpensive and therefore accessible to smaller flight schools. Visual cueing, vibratory cueing, and sound cueing systems that were developed for the CURVS are the topics of discussion in this thesis.

For the visual system, an asymmetric 220 with a cylindrical wraparound display as well as an image generator that runs X-Plane smoothly and a custom scenery for Waterloo Airport were designed and implemented. A standalone vibratory cueing system that shakes the pilot seat, was designed, implemented, and analyzed. Also, a 5.1 channel sound system was installed on the simulator and was tuned with the intention to replicate the exact Cessna 172 engine noise.

Finally, multiple flight tests were performed to obtain simulator tuning data. A quantitative comparison between the aircraft and the simulator, and as well as a qualitative evaluation of the simulator is also presented. Results confirm that the cueing system satisfy their design requirements. 
I would like to dedicate my work to the people who directly and indirectly contributed in it:

To my parents Hooshang and Mitra, who gave me the chance to live in this amazing world, and their support which has been the best gift in my life.

To my only and lovely sister Mahsa, who I seemingly missed while I stayed in Canada.

To my uncle Farhang and his wonderful wife Gitty, who compassionately helped me through my masters.

And most importantly to Rob, my friendliest teacher, who brought this opportunity for me and helped me to feel closer to my dreams. 


\section{Acknowledgments}

I'd like to acknowledge the staff, faculty, students, and the industry partner who made this amazing research opportunity possible. Robert Langlois, my patient and stunning supervisor; Robert Connors, graceful CEO of Waterloo Wellington Flying Centre who was our industry partner; Brent Cameron, who I carried out the CURVS project with and he provided many of the pictures; Stefano Sandri, a merciful volunteer student who helped me to build the screen; Chris Hobbs, the amazing pilot and generous friend of mine who kindly helped me for flight tests; and Harry Zanin for his useful comments. My special thanks goes to all the participants whose contribution by patiently donating their time into the simulator flight tests, made this research, that was my childhood passion, happen. 


\section{Table of Contents}

Abstract $\quad$ ii

Acknowledgments $\quad$ iv

Table of Contents $\quad$ v

List of Tables $\quad$ ix

List of Figures $\quad$ X

List of Acronyms xvi

1 Introduction 1

1.1 Pilot Cues and FSTD . . . . . . . . . . . . . . . 1

1.1 .1 Cues ............................... 1

1.1.2 Flight Simulation Training Device . . . . . . . . . . . . . 3

1.2 Background and Review of Flight Simulators . . . . . . . . . . . 6

1.2.1 Flight Training History . . . . . . . . . . . . . . 6

1.2 .2 Visual Cueing . . . . . . . . . . . . . . . 11

1.2.3 Motion and Vibration . . . . . . . . . . . . . . . 14

1.2.4 Sound in Flight Simulators . . . . . . . . . . . . . . . 15

1.3 CURVS Project . . . . . . . . . . . . . . . . 16

1.3.1 Objectives of the Project and the Thesis . . . . . . . . 16 
1.4 Outline and Thesis Overview . . . . . . . . . . . . 18

2 Visual Cueing System $\quad 20$

2.1 Main Aspects of a VCS . . . . . . . . . . . . . . . 20

2.1.1 FOV Versus Screen Resolution . . . . . . . . . . . . . . . . . 21

2.1 .2 Parallax and Collimation . . . . . . . . . . . . . . . 23

2.1 .3 Scenery Versus Frame Rate . . . . . . . . . . . . . . 25

2.2 CURVS Visual Cueing System . . . . . . . . . . . . . 26

2.2 .1 Pre-design Procedures $\ldots \ldots \ldots \ldots$

2.2 .2 The Image Generator . . . . . . . . . . . . . . . . . . . . 32

2.2 .3 Projection System _ . . . . . . . . . . . . . . . 37

2.3 Customized Add-on Scenery . . . . . . . . . . . . . . . . . . 51

2.3.1 Aerial Data Collection . . . . . . . . . . . . . . . 53

$2.3 .2 \quad$ World Editor . . . . . . . . . . . . . . . 57

2.3 .3 SketchUp . . . . . . . . . . . . . . . . . . . 58

2.3.4 Aircraft Component Modification . . . . . . . . . . . 58

2.3.5 Resulting Scenery . . . . . . . . . . . . . . . 60

2.4 Tuning and Evaluation . . . . . . . . . . . . . 65

2.4.1 Geometrical Correction and Soft-edge Blending . . . . . . 65

2.4 .2 Screen Resolution . . . . . . . . . . . . . . . . . 68

2.4 .3 Parallax Evaluation . . . . . . . . . . . . . . . . . 69

3 Vibratory and Auditory Cueing $\quad 71$

3.1 Design . . . . . . . . . . . . . . . . . . . 71

3.2 Equipment Assembly . . . . . . . . . . . . . . . . . . 75

3.3 Sound Tuning $\ldots \ldots \ldots \ldots \ldots$ 
4 Experiment and Analysis $\quad 82$

4.1 Cessna 172 Tests . . . . . . . . . . . . . . . . . . . 82

$4.1 .1 \quad$ First Flight . . . . . . . . . . . . . . . . . . . . 83

4.1 .2 Second Flight . . . . . . . . . . . . . . . . . . . . . . 85

4.1 .3 Third Flight . . . . . . . . . . . . . . . . . . . 88

4.1.4 Simulator Quantitative Test . . . . . . . . . . . . . 90

4.2 Results . . . . . . . . . . . . . . . . . . . . . 91

4.2.1 Summary of the Tests . . . . . . . . . . . . . . . . 91

4.2 .2 Sound Pressure Level . . . . . . . . . . . . . . . . . . . . . . . 91

4.2 .3 Light Intensity . . . . . . . . . . . . . . . . . . . . . 93

4.3 Frequency Analysis . . . . . . . . . . . . . . . . . . . . . . 94

4.3.1 Vibration Data and Analysis . . . . . . . . . . . . . 96

4.3.2 Sound Data and Analysis . . . . . . . . . . . . . . . 101

4.4 Qualitative Evaluation . . . . . . . . . . . . . . 105

$\begin{array}{lll}5 & \text { Conclusion } & 113\end{array}$

5.1 Summary and Conclusion . . . . . . . . . . . . . . . 113

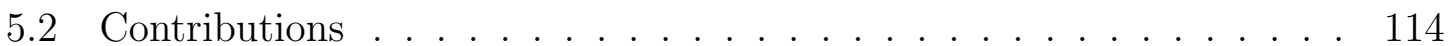

5.3 Future work . . . . . . . . . . . . . . . . . 115

$\begin{array}{ll}\text { References } & 117\end{array}$

$\begin{array}{lll}\text { Appendix A Hardware and Software Specifications } & 121\end{array}$

Appendix B Fabric Factsheet and the Screen Tubes 126

Appendix C Immersive Calibration Pro Properties 134

Appendix D Flight Tests Weather Data 136 
Appendix G Matlab Codes for PSD Analysis 156

G.1 Vibration Analysis Matlab Code . . . . . . . . . . . . . . . . 157

G.2 Sound Analysis Matlab Code . . . . . . . . . . . . . . . 160 


\section{List of Tables}

4.1 Summary of flight tests . . . . . . . . . . . . . . . . . 91

4.2 Sound pressure level in different manoeuvres and engine RPMs for the CURVS and the aircraft . . . . . . . . . . . . . . . . 92

4.3 Brightness level comparison of the simulator and the actual aircraft. . 93

4.4 Questionnaire results - Participants 1 to 4. . . . . . . . . . . 111

4.5 Questionnaire results - Participants 5 to 8. . . . . . . . . . . . . 112

H.1 List of qualitative test participants. . . . . . . . . . . . . . 163 


\section{List of Figures}

1.1 A modern full flight simulator. The picture is credited to Mechtronix. 4

1.2 Alsim flight simulation training device in WWFC with 208 degrees horizontal FOV. Photographed by Brent Cameron. . . . . . . . . 5

1.3 A Link flight trainer at the Western Canada Aviation Museum. . . . 7

1.4 Customized hardware image generator of a Mechtronix FSTD. . . . . 8

1.5 Microsoft Flight Simulator 1. . . . . . . . . . . . . . . . . . . 9

1.6 3D cockpit and simulation of detailed systems of an MD-90 aircraft in Microsoft Flight Simulator X. . . . . . . . . . . . . . . . . . 10

1.7 Customized projector of a Mechtronix FSTD with shutters. . . . . . . 11

2.1 PPI comparison: Full HD image on the left and 4K image on the right. 22

2.2 Arcmin is an angle by which the human eye's resolution is defined. . . 22

2.3 Parallax effect. Different POVs see different objects. . . . . . . . . . . 24

2.4 Parallax effect. When the scene is focused at the centre of the screen, the pilot feels that the aircraft is yawing to the left. . . . . . . . . . . 24

2.5 Real-time frames. Calculations must be done within the time limit. . 26

2.6 Panaromic view of the front window in a Cessna 172 during cruise flying. 29

2.7 Touch-and-go with the Cessna 172 during flight tests in the Gatineau aerodrome. . . . . . . . . . . . . . . . . . 30

2.8 A standard circuit pattern chart. . . . . . . . . . . . . . 32

2.9 Cessna 172 flying in downwind leg at the Rockcliffe aerodrome. . . . . 33 
2.10 OSV in a Cessna 172 flying on the downwind leg ready to turn to base leg at the Rockcliffe aerodrome. . . . . . . . . . . . . . . . 33

2.11 CURVS visual system diagram. . . . . . . . . . . . . . . 35

2.12 A Cessna 172 flying in a sunset scenario in X-Plane 10 . . . . . . . . 36

2.13 Projector's image geometric parameters. . . . . . . . . . . . . 38

2.14 A projector's throw angles. . . . . . . . . . . . . . . 39

2.15 All the screen parameters with $10 \%$ overlap of the images. . . . . . . 40

2.16 Simulator cabin fitting well inside the screen and the room dimensions. 43

2.17 Top view of the projection setup with $10 \%$ overlap of the images. . . 44

2.18 Shadow analysis for the CURVS visual system. . . . . . . . . . . 44

2.19 Shadow analysis in the front view. Note the reflections that must be eliminated. . . . . . . . . . . . . . . . . 45

2.20 FlexiWhite fabric and the bungees for the screen. . . . . . . . . . . 46

2.21 FEA analysis of the frame. Note the extra top bar was employed to support the projector mount. . . . . . . . . . . . . . . . . . 47

$2.223 \mathrm{D}$ model of the connectors (Kee Klamps). . . . . . . . . . . . . . . 48

2.23 Aluminum component hammered and screwed to hold the curved tubes accurately $(3 \mathrm{D}$ model on the left) . . . . . . . . . . . . . . . . 49

2.24 Plywood used for mounting the projectors. . . . . . . . . . . . . . . 49

2.25 Final assembly of the CURVS screen with all the team members. . . . 50

2.26 Cardboard mockup of the CURVS simulator. . . . . . . . . . . . 51

2.27 C172 aircraft window measurement. . . . . . . . . . . . . . 52

2.28 Open Street Map database converted into X-Plane and objects like roads, rivers, and trees generated the exact position on top of the satellite images. . . . . . . . . . . . . . . . . . . 55

2.29 X-Plane image generation flow chart. . . . . . . . . . . . 56

2.30 WED interface. . . . . . . . . . . . . . . . 57 
2.31 The design of the Waterloo International Airport control tower using SketchUp. . . . . . . . . . . . . . . . . . . 59

2.32 The dynamic wing view in the CURVS visual system. . . . . . . . . 60

2.33 Waterloo airport (CYKF) custom-made scenery overview. Note the framerate is 60 FPS in an area with high object density. . . . . . . 61

2.34 Waterloo airport (CYKF) main terminal apron custom scenery. . . . 61

2.35 A view from the WWFC parking lot of the Waterloo Airport (CYKF) custom scenery looking toward the control tower. . . . . . . . . . . . 62

2.36 Waterloo Airport (CYKF) WWFC main office building custom scenery. 62

2.37 A picture of the WWFC parking lot (real) . . . . . . . . . . 63

2.38 The same spot as Figure 2.37 in the simulated environment. . . . . . 63

2.39 Waterloo airport (CYKF) WWFC main office building custom scenery. 64

2.40 Waterloo airport (CYKF) custom Runway 26 with PAPI lights, ODALS approach lighting system, and animated wind sock. . . . . . 64

2.41 Manual calibration of the images using Immersive Calibration Pro. . . 66

2.42 Calibrated screen test pattern. . . . . . . . . . . . . . 68

2.43 Final result of the calibrated screen with blended images. . . . . . . . 68

2.44 The calculated resolution of the CURVS VCS. . . . . . . . . . . . 69

2.45 Parallax angle of the CURVS VCS which is 8.24 degrees. . . . . . . . 70

3.1 Dual channel stereo speaker previously used in the VectorSim. . . . . 72

3.2 Sound and vibration cueing system diagrams in the CURVS. . . . . . 73

3.3 The Buttkicker package. . . . . . . . . . . . . . . . . 74

3.4 The sound system for the CURVS. . . . . . . . . . . . . . . 74

3.5 The pilot seat detached from the cabin. . . . . . . . . . . . 75

3.6 The aluminum plate that was built for the Buttkickers on the left and then attached to the seat on the right. . . . . . . . . . 76 
3.7 The metal plate that was drawn for the Buttkickers attachment (all the units are in inches). . . . . . . . . . . . . . . . . . 77

3.8 The rubber isolators on the left and attached to the seat on the right. 77

3.9 The upgraded seat re-attached to the simulator cabin. . . . . . . . 78

3.10 The 5.1 channel surround sound system. . . . . . . . . . . . . . 78

3.11 Plane-maker settings for the sound files. . . . . . . . . . . . . 81

3.12 The Audacity interface. . . . . . . . . . . . . . . . . . 81

4.1 The Cessna 172 flown for the flight test. . . . . . . . . . . . . . . 83

4.2 A GoPro Hero 2 camera attached to the pilot's head. . . . . . . . . . 83

4.3 Panoramic view of a C172 cockpit. . . . . . . . . . . 84

4.4 Gear view of the C172. . . . . . . . . . . . . . 85

4.5 Gulf Coast Data Concepts X16-2 portable USB accelerometer. . . . . 86

4.6 Sony digital audio recorder that was used to record engine noise. . . . 86

4.7 Digital sound pressure level meter. . . . . . . . . . . . . . . 87

4.8 Spot meter that measures light intensity in EV. . . . . . . . . . . . 88

4.9 Night flight over Ottawa, approaching on Runway 14 at CYOW. . . . 89

4.10 The accelerometer's axis and its orientation in the test. . . . . . . . . 89

4.11 Welch method segments with $50 \%$ overlapping of the sequential segments. 95

4.12 Accelerometer orientation. . . . . . . . . . . . . . . . 96

4.13 Acceleration versus time of the aircraft seat vibration in all directions while the engine was idling at 750 RPM. . . . . . . . . . . . . . . 98

4.14 PSD analysis of the aircraft seat vibration in all three coordinate directions while the engine was idling at 750 RPM. . . . . . . . . . . . 99

4.15 Acceleration versus time of the simulator seat vibration in all directions while the engine was idling at 750 RPM. . . . . . . . . . . . . . . 99

4.16 PSD analysis of the simulator seat vibration in all three coordinate directions while engine was idle running at 750 RPM. . . . . . . . . 100 
4.17 PSD comparison of the simulator and aircraft seat vibrations in the $\mathrm{Y}$ direction at 750 RPM. . . . . . . . . . . . . . . 100

4.18 Simulator sound recording process. . . . . . . . . . . . . . . 101

4.19 Normalized sound data in the time domain for the aircraft at 750 RPM.103

4.20 PSD analysis of the aircraft sound in the low frequency range at 750

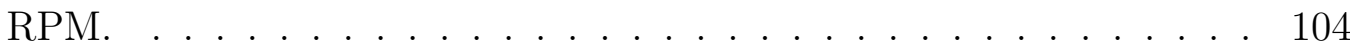

4.21 Normalized sound data in the time domain for the simulator at 750

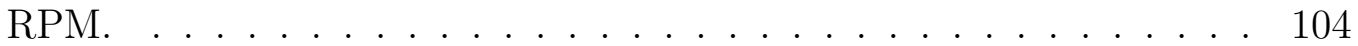

4.22 PSD analysis of the simulator in the low frequency range at 750 RPM. 105

4.23 PSD analysis of the simulator and the aircraft sound at 750 RPM. . . 105

4.24 Full range PSD analysis of the simulator versus the actual aircraft sound.106

4.25 One of the participants flying circuits at CYKF. . . . . . . . . . 107

4.26 The tablet implemented to replicate the six pack instruments. . . . . 108

4.27 The circuit pattern at CYKF flown by one of the participants. . . . . 108

4.28 The results of the rating questions. . . . . . . . . . . . . . . 110

F.1 PSD analysis of the vibration of the aircraft taxiing at 1000 RPM. . . 151

F.2 PSD analysis of the vibration of the simulator taxiing at 1000 RPM. . 151

F.3 PSD analysis of the vibration of the simulator and aircraft in the $\mathrm{Y}$ direction taxiing at 1000 RPM. . . . . . . . . . . . . . . . . . 152

F.4 PSD analysis of the vibration of the aircraft cruising at 2400 RPM. . 152

F.5 PSD analysis of the vibration of the aircraft cruising at 2400 RPM. . 153

F.6 PSD analysis of the vibration of the simulator and the aircraft in the Y direction cruising at 2400 RPM. . . . . . . . . . . . . . . . 153

F.7 Full-range PSD analysis comparing the sound of the simulator and the aircraft taxiing at 1000 RPM. . . . . . . . . . . . . . . 154

F.8 Low-frequency range PSD analysis comparing the sound of the simulator and the aircraft taxiing at 1000 RPM. . . . . . . . . . . . 154 
F.9 Full-range PSD analysis comparing the sound of the simulator and the aircraft cruising at 2400 RPM. . . . . . . . . . . . . . . . . 155

F.10 Low-frequency range PSD analysis comparing the sound of the simulator and the aircraft cruising at 2400 RPM. . . . . . . . . . . . 155 


\section{List of Acronyms}

\begin{tabular}{ll} 
Acronyms & Definition \\
\hline \hline arcmin & Arc-minutes \\
ACC & Acceleration \\
ADL & Applied Dynamics Laboratory \\
AI & Attitude Indicator \\
ASI & Airspeed Indicator \\
ATC & Air Traffic Control \\
ATR & Aviation Training Resources \\
BET & Blade Element Theory \\
C172 & Cessna 172 \\
CFS & Canada Flight Supplement \\
CSV & Comma Separated Values \\
COTS & Commercial Off The Shelf \\
CURVS & Carleton University Redeveloped Vector Simulator \\
& \\
& \\
& \\
&
\end{tabular}




$\begin{array}{ll}\text { CYRO } & \text { Rockcliffe Airport } \\ \text { CYOW } & \text { Ottawa/Macdonald Cartier International Airport } \\ \text { DCS } & \text { Double Curved Screens } \\ \text { DSF } & \text { Distribution Scenery Format } \\ \text { EFIS } & \text { Electronic Flight Instrument System } \\ \text { ETU } & \text { Easy To Use } \\ \text { FAA } & \text { Federal Aviation Administration } \\ \text { FDM } & \text { Flight Dynamic Model } \\ \text { FEA } & \text { Finite Element Analysis } \\ \text { FFS } & \text { Full Flight Simulator } \\ \text { FFT } & \text { Fast Fourier Transform } \\ \text { FMC } & \text { Flight Management Computer } \\ \text { FOV } & \text { Field of View } \\ \text { FPS } & \text { Frames Per Second } \\ \text { FSTD } & \text { Flight Simulation Training Device } \\ \text { GPU } & \text { Graphical Processing Unit } \\ \text { Fis } & \text { Glass Reinforced Plastic } \\ \text { Feographical Information System }\end{array}$




$\begin{array}{ll}\text { HI } & \text { Heading Indicator } \\ \text { ICP } & \text { Immersive Calibration Pro } \\ \text { IDP } & \text { Immersive Display Pro } \\ \text { IFR } & \text { Instrument Flight Rules } \\ \text { IR } & \text { Instrument Rating } \\ \text { IOS } & \text { Instructor Operating Station } \\ \text { ICAO } & \text { International Civil Aviation Organization } \\ \text { LHS } & \text { Left Hand Side } \\ \text { LH } & \text { Left Handed } \\ \text { MSFS } & \text { Microsoft Flight Simulator } \\ \text { MDF } & \text { Medium Density Fibre-board } \\ \text { ND } & \text { Navigation Display } \\ \text { OCE } & \text { Ontario Centre of Excellence } \\ \text { OSM } & \text { Open Street Map } \\ \text { OSV } & \text { Over Shoulder View } \\ \text { OTF } & \text { Over The Field } \\ \text { Othe Window }\end{array}$




\begin{tabular}{|c|c|}
\hline PPI & Pixel Per Inches \\
\hline PSD & Power Spectral Density \\
\hline RPM & Revolutions Per Minute \\
\hline SPL & Sound Pressure Level \\
\hline ST & Short Throw \\
\hline SWOOP & Southwestern Ontario Ortho-photography Project \\
\hline TER & Transfer of Training \\
\hline TC & Transport Canada \\
\hline $\mathrm{TR}$ & Throw Ratio \\
\hline UDP & User Datagram Protocol \\
\hline USGS & United States Geological Service \\
\hline VASI & Visual Approach Slope Indicator \\
\hline VCS & Visual Cueing System \\
\hline VFR & Visual Flight Rules \\
\hline VFOV & Vertical Field of View \\
\hline VSI & Vertical Speed Indicator \\
\hline WAV & Waveform Audio Format \\
\hline WED & World Editor \\
\hline WWFC & Waterloo Wellington Flight Centre \\
\hline
\end{tabular}




\section{Chapter 1}

\section{Introduction}

Flight simulation offers opportunities for providing enhanced flight training more costeffectively. Simulation devices adequately immerse the trainee in a realistic simulated flight environment in which not only they can learn how to fly in a safe manner, but also the practices learnt can be counted towards the required hours for acquiring pilot licenses. This thesis considers the design, analysis, implementation and testing of a professional flight training device (FSTD) using Commercial Off The Shelf (COTS) components, in an attempt to reduce the cost of building these expensive machines.

The introduction is arranged in four sections. First, cueing systems and FSTDs are introduced, then prior research in the literature is discussed. Next, the Carleton University Redeveloped Vector Simulator (CURVS) project is described and finally the content of this thesis is outlined.

\subsection{Pilot Cues and FSTD}

\subsubsection{Cues}

Humans perceive the world through stimulation of their senses that have been evolved

over millions of years to function in their most optimal way. Through the sense of 
hearing, for instance, sound is converted into audio signals as stimuli and those signals are fed into the brain so that the brain can model some of the phenomena that occur in the surrounding environment. Albeit, a combination of multiple inputs (multiple cues) through different senses are required for the brain to make a model from the world outside and respond accordingly. $[1]^{1}$

In flight simulation, these stimuli are referred to as cues. For engineers, since the simulation of every detail within the flight environment is not easy, the study of human senses may help to prioritize the most crucial cues and eliminate the ones that have less impact on situational awareness. Also, as the human perception is sophisticated, for some cues (vibration and sound) it is possible for the simulator to behave slightly different than the actual aircraft while the pilot perceive the same feeling. In fact, considerable savings can be made by eliminating unnecessary cues, however, it is always important for engineers to keep the fidelity (realism) of the simulation at a sufficiently high level for it to be meaningful. [1]

Chemical, thermal, photochemical and mechanical stimuli are the main four cues that humans perceive. Where chemical cues generate taste and smell, thermal cues provide the feel of temperature, for flight simulation, is usually the ambient operating temperature of the cockpit. Photochemical systems help to sense colour and brightness through the eyes. Mechanical cues provide multiple environments to detect sound, gravity, and motion by using the body's vestibular system. Also, position and movements of joints and pressure applied to the body are sensed through kinaesthetic senses and likewise pressure on the skin by the tactile sense. Among these four cues, photochemical and mechanical cues are the most dominant ones that affect flight simulation fidelity. [1]

Generally, a flight simulator is a combination of multiple cues working simultaneously to imitate the flight environment. A flight simulator consists of an instrument

\footnotetext{
${ }^{1}$ Citations outside the punctuation refer to the whole paragraph.
} 
panel, control sticks (or yokes), throttle and mixer levers, flaps handle, trim wheel, circuit breakers, various switches, etc. mimicking the cockpit environment. Visuals are provided by either single or multiple projectors (or LCD panels) working together to convey different fields of view (FOV). Projectors are also responsible for rendering runway, taxiway, terrain, and miscellaneous objects (scenery) on screen, alongside replicating the flight dynamic model (FDM) of the aircraft which is calculated by a computer in response to pilot inputs.

To duplicate the motion and vibration sensed in the cockpit through different manoeuvres, motion platforms can be implemented to stimulate the vestibular sense. All the accelerations, velocities, and positions are calculated in real-time by computers and an associated washout or tilt-coordination algorithm maps real vehicle motions to the smaller ranges of motion that are achievable with motion platforms. The same way, all the sounds and noises are reproduced and synthesized by computers and regenerated through provided speakers. Furthermore, emulating the force-feedback feel of flight controls for the pilot is important as they affect the flight handling and pilot response during a real flight. Force feedback is provided by control loading systems (haptic systems) that are embedded into the control yoke or stick as well as the rudder pedals using rotary motors or electromagnetic actuators. Figure 1.1 is an illustration of a full-flight simulator that is an exact replica of a Boeing 737 cockpit.

\subsubsection{Flight Simulation Training Device}

Flight simulators are categorized into two types: Full Flight Simulators (FFSs) and Flight Simulation Training Devices (FSTDs) each having their own classification system. The most apparent difference between the two is that FFSs have a motion platform, whereas FSTDs do not require motion. Also, the level of fidelity is much higher in FFSs than FSTDs by replicating all the systems in detail resembling the 

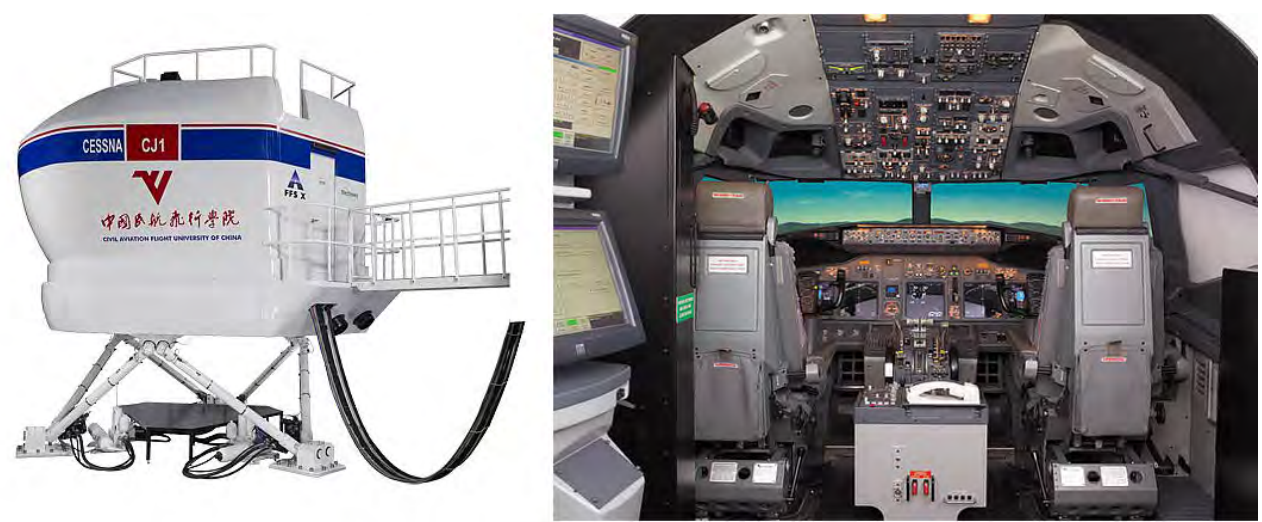

Figure 1.1: A modern full flight simulator. The picture is credited to Mechtronix.

actual aircraft. FFSs, by which airline and helicopter pilots are trained, are classified as level A to D based on implementation of different cueing systems and their operational quality with various applications and costs, with level D representing the highest. FSTDs are classified in levels 2 to 7 . Levels 2 and 3 adopt a generic cockpit; nevertheless, levels 4 through 7 represent the cockpit of a specific type of aircraft. Within the generic or specific category, as the level of FSTD increases, its complexity increases correspondingly. [2] Some companies, such as TRU Simulation [3], sell FSTDs that can be upgraded to FFSs by subsequently adding a motion platform to the simulator.

The other difference between FSTDs and FFSs is the quality of each implemented cueing system. For instance, FFSs are required to have expensive collimated visual displays to visualize the objects with an accurate size and diminishing the parallax effect; whereas, having visual cueing (and also motion cueing) is only considered an asset for FSTDs. Moreover, utilization of an authentic aerodynamic model verified by flight tests is not required for FSTDs. [2]

FSTDs are mostly used in flight schools and universities since they are more affordable than FFSs. FFSs may cost multi-million dollars whereas FSTDs cost somewhere in the range of a few hundred thousand dollars depending on the fidelity and the usage. The cost of a high-fidelity FSTD is up to three times the price of 


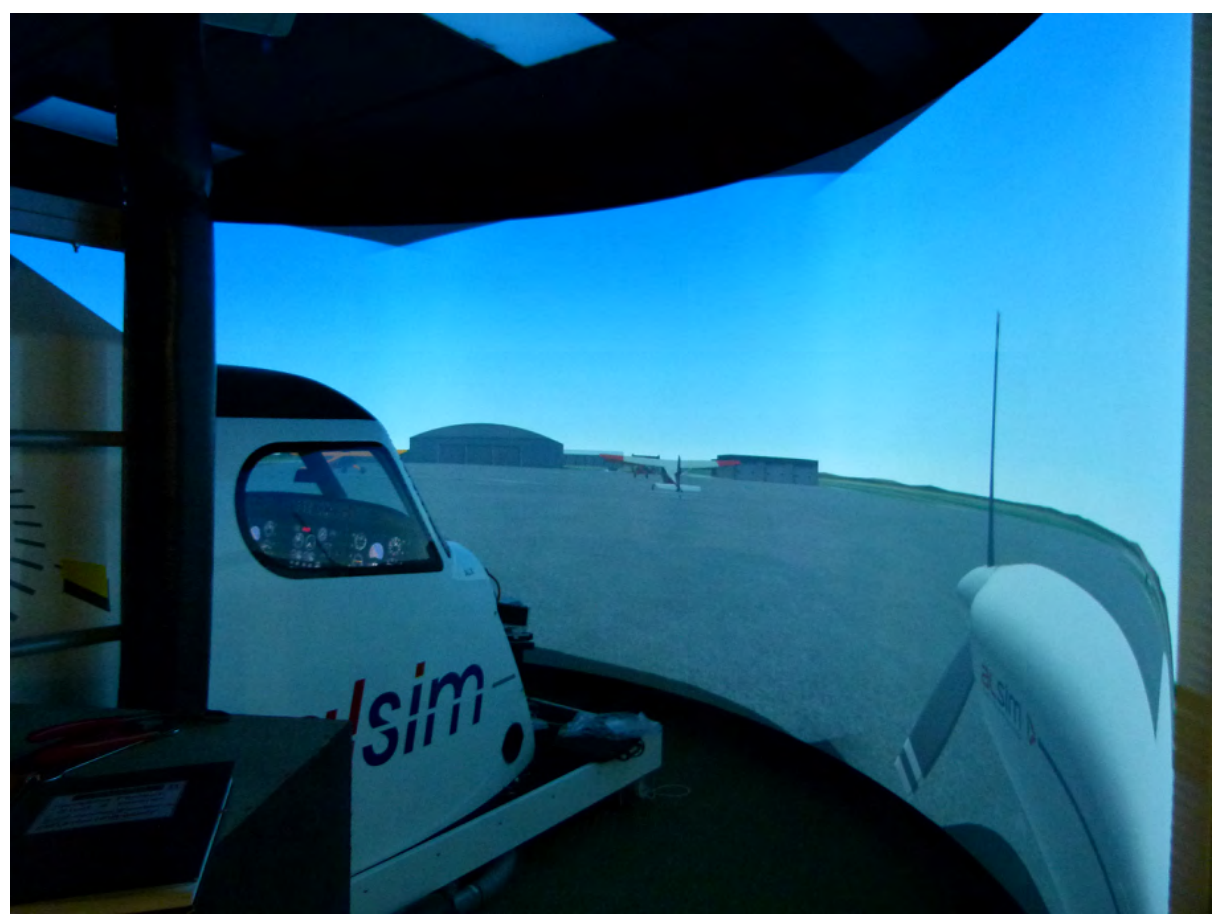

Figure 1.2: Alsim flight simulation training device in WWFC with 208 degrees horizontal FOV. Photographed by Brent Cameron.

a new plane (depending on the manufacturer and model) and therefore flight clubs often tend to go without them [4]. Figure 1.1 shows a level 2 high-fidelity flight simulation training device manufactured by Alsim in France, recently installed at Waterloo Wellington Flight Centre at a cost of half a million dollars [5].

An FSTD could be used in a flight school for a variety of applications including: cockpit familiarization, ab-inito training, practicing for private pilot license (PPL), practicing for commercial pilot license (CPL), practicing for instrument rating (IR), etc. Students can get familiar with the basics of flight and experience different scenarios such as take off, traffic circuits or traffic patterns, cruise flying, landing, or emergency situations like engine failure, instrument loss, or control malfunctions. Also, one important skill that is acquired by practicing in an FSTD is quick decision making, particularly in critical conditions. [6]

The focus of this thesis is mainly on analysis, design, and implementation of visual 
cueing, auditory cueing, and vibratory cueing (as a stand alone cueing system) that was implemented in a high fidelity $\mathrm{FSTD}^{2}$ with a lower cost by taking advantage of COTS products to the extend possible. In the next section, flight training history and background related to each cueing system will be discussed.

\subsection{Background and Review of Flight Simulators}

\subsubsection{Flight Training History}

Since the invention of the first human controllable airplane by the Wright brothers aviation pioneers, the importance of pilot training was realized. First, it was limited solely to advise pilots on how to fly planes on the ground. Then some training devices started to appear, enabling pilots to practice the effects of controls on the ground [1].

Despite the rapid advancements in the early years of aeronautics, the handling qualities of early aircraft were poor and many pilots were having a hard time to learn how to fly. It is claimed that more lives were lost in training than in combat during the First World War this led to recognition of the importance of appropriate pilot training before heading into the air. [7]

The Link Trainer or the 'Blue Box' is known to be the first flight training device officially used to train pilots. The Link Trainer was built by Edward Link in the 1920s in New York and was used as a key pilot training aid by almost every combatant nation during the Second World War. Link is also known as the founder of modern day flight simulation. The Blue Box enabled a significant part of instrument flight training to be conducted in a ground-based trainer. Figure 1.3 shows a Blue Box. [8]

Later on, in part enabled by advancements in control engineering, and the advent of analogue computing, differential equations underlying flight models could be solved by electronic circuits. At this time, despite the significant improvements to the fidelity

\footnotetext{
${ }^{2}$ A high-fidelity flight simulator is not necessarily a high-level FSTD.
} 


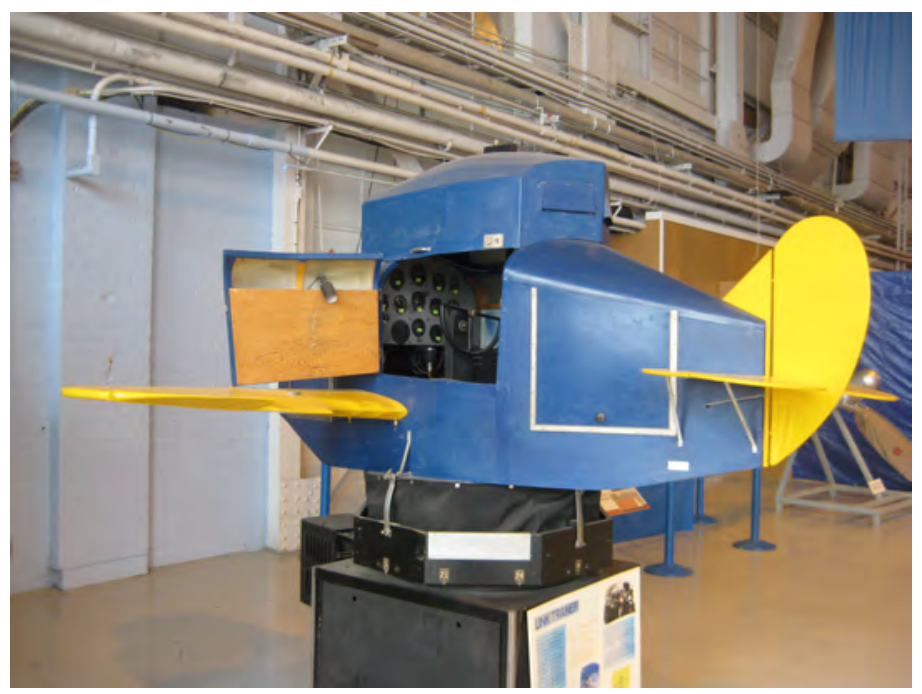

Figure 1.3: A Link flight trainer at the Western Canada Aviation Museum.

of flight models, progress in motion and visual cueing was much slower. The major operation of simulators was to practice instrument flying conditions due to the lack of a visual system.

When microelectronic chips emerged in 1985, it revolutionized the computer industry and, it became possible to solve the equations of motion at $60 \mathrm{~Hz}$ using only a single processor. At that time, attention shifted to the development of visual systems [9]. Terrain, airfield, and urban areas could be simulated by using a digital geographical database. Also, by the 1990s, it became possible to carefully blend the images generated by three or more projectors to achieve a continuous 'wrap-around' display [1]..$^{3}$

The flight simulator industry has always demanded more computation power than what currently existed in the market. In fact, sophisticated algorithms are solved in real-time to run the flight model and the scenery using advanced computer hardware, as illustrated in Figure 1.4. Also, aircraft instruments are replicated and behave like the actual aircraft. Therefore, made-to-order hardware and software engineering have always been an inevitable tradition in the flight simulation business.

\footnotetext{
${ }^{3}$ For a complete history of flight simulation, refer to the Chapter 1 of the Reference [1].
} 


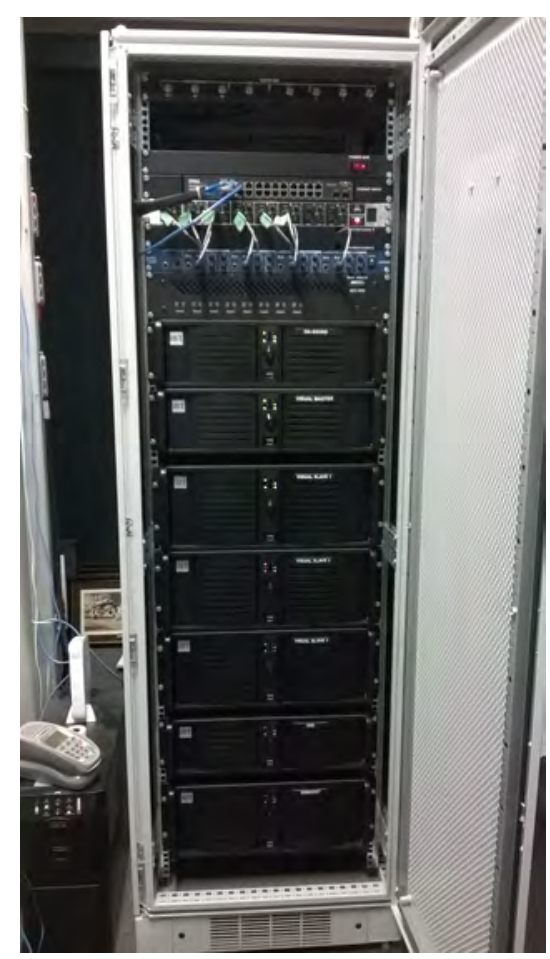

Figure 1.4: Customized hardware image generator of a Mechtronix FSTD.

Although flight simulator companies tend to employ their own simulation software, after a few preliminary flight simulator computer programs were released for Apple II and TRS-80 computers in the 1970s, Microsoft started to develop new flight simulator software named Microsoft Flight Simulator (MSFS) that could be flown by users using their home-based PCs, as shown in Figure 1.5. MSFS was the first sophisticated piece of software that could replicate different flight manoeuvres in a 3D graphics environment. The project was so successful that Microsoft progressively released a newer version every few years; with the latest version being Microsoft Flight Simulator X or FSX for short. Microsoft Flight Simulator opened a new gateway for aviation lovers bringing them the opportunity to learn, practice, and fly their own favourite simulated plane at their local airport using a single PC, mouse, keyboard, and an affordable desktop joystick. By stepping into the internet era, online communities for home flight simulators, including websites and forums, started to emerge and 


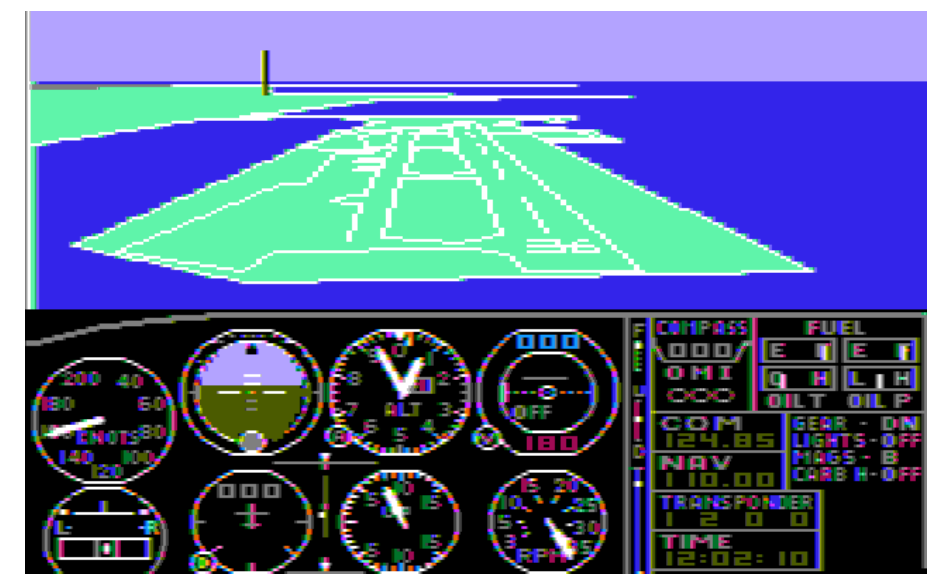

Figure 1.5: Microsoft Flight Simulator 1.

many developers from all around the world started to contribute to expanding MSFS capabilities by designing their own add-on aircraft, scenery, sound, plugins, etc. Also, some professional developers started to sell their hardware and software online, adding more and more realism to home-user flight simulators. The recent versions of MSFS, thanks to the rise of the add-on market, are capable of simulating most of the complex aircraft systems including the electronic flight instrument system (EFIS), primary flight display (PFD), navigation display (ND), flight management computer (FMC), etc. in a highly realistic environment so that even real airline pilots practice at home using desktop simulators (refer to Figure 1.6). [10,11]

One challenge in building a professional flight simulator is to provide a visual system that can generate high-quality images on a seamless and immersive display. This can be done by using custom-made projectors and curved wrap-around screens. The problem arises as projectors have a limited throw angle and also they are not designed to throw the image on a curved screen without causing geometrical distorsions. Using customized software technologies, it is possible to mitigate the geometrical distortion of the image. Also by implementing multiple projectors in a row it has been demonstrated that one can increase the field of view; however, blending the images of different visual channels to get a smooth and homogenous portrait is the next 


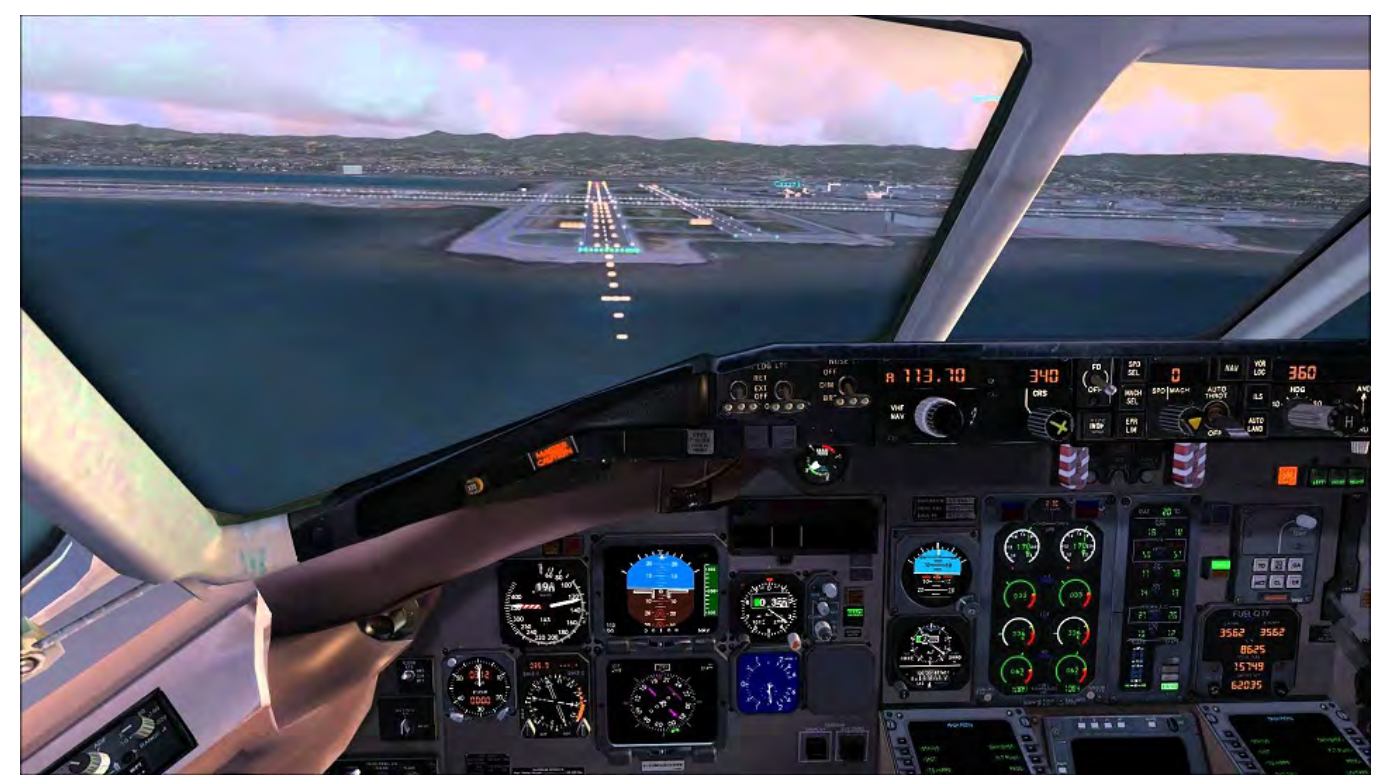

Figure 1.6: 3D cockpit and simulation of detailed systems of an MD-90 aircraft in Microsoft Flight Simulator X.

dilemma. Once the projectors are lined up there will be regions in which the images are blended. Since there is two beams of light on the blending areas, the brightness of that region becomes doubled. This has been alleviated by making use of custommade shutters in front of the projectors lenses to make artificial shadows at the edges of the images, as shown in Figure 1.7.

Nowadays, with the advancement of computers, particularly in gaming, both software and hardware wise, building an FSTD by using COTS products is completely feasible, while also significantly reducing the total cost. Two M.A.Sc students in the Applied Dynamics Laboratory at Carleton University recently built a cost-effective FSTD named 'KatanaSim' mostly from COTS components combined with an actual DA-20 Katana aircraft fuselage. The goal was to assess the feasibility of this approach and it was found to be viable. $[12,13]$

In the following section, each cueing system is discussed individually. 


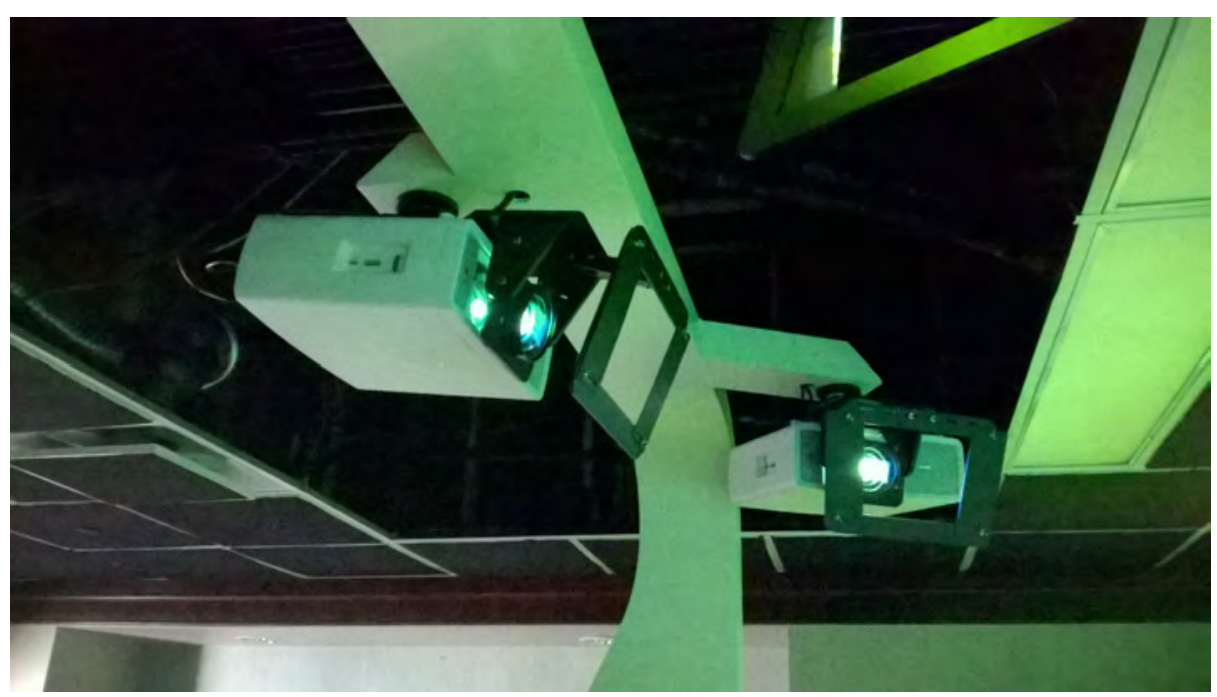

Figure 1.7: Customized projector of a Mechtronix FSTD with shutters.

\subsubsection{Visual Cueing}

Despite of the fact that Transport Canada's Aeroplane and Rotorcraft Simulator Manual [2] states that having a visual cueing system in an FSTD is NOT mandatory, implementing one brings many advantages [2]. In fact, most modern commerciallyavailable FSTDs are sold with visual cueing systems due to high customer demands and the benefits they offer. $[14,15]$

Formerly, FSTDs were used to make the introductory student pilots conversant with the cockpit perimeter and basic aircraft avionic system functionality [16]. In a state-of-the-art FSTD; however, pilots now have the opportunity to learn basic ab-initio training by practicing basic manoeuvres under Visual Flight Rules (VFR) conditions prior to being in a real aircraft and also have the opportunity to rehearse instrument navigation under Instrument Flight Rules (IFR) conditions where limited or no visual environment is available such as at night or on a foggy day [17].

The minimum visual FOV required in an FSTD (if implemented at all), according to the Federal Aviation Administration (FAA)regulatory manual for FSTDs, is 18 
degrees vertically and 20 degrees horizontally [18]. Notwithstanding, by taking advantage of COTS projectors and blending software available today, building a curved display with 180 degrees horizontal FOV is very common among non-professional flight simulator hobbyists $[19,20]$. Therefore, achieving higher horizontal FOVs is not inconceivable (without losing the quality incurring a substantial much increase in cost). One advantage of a greater FOV is that it makes it possible for the pilot to practice over-shoulder viewing to spot the runway before turning on to the base leg during a circuit (traffic pattern) [21]. This is one of the main achievements in this thesis that will be discussed in detail in Chapter 2 .

One important factor in a high quality visual cueing system is the frame rendering speed. Studies have shown that starting at about 50 frames per second, users subconsciously forget that they are looking at a simulator and begin thinking they are actually flying $[1,22]$. In one study in an air traffic control (ATC) simulator, thirteen active air traffic controllers viewed high-dynamic-fidelity simulations of landing aircraft and decided whether the aircraft would stop before the end of the runway, as if to be able to make a runway turnoff. The required frame rate appeared to range from 30-60 FPS, but definitive recommendations require further testing at a higher rate in the range of 45-60 FPS [23]. Generally, low frame-rate is associated with visual delays and simulator visual delay has been tied to an increase in pilot workload [24,25], deterioration of pilot performance [26], and a cause of simulator sickness [27].

Apart from framerate, practical experience and behavioural research have shown that for pilots to perform well in a simulator in which the visual scene is complex (the existence of contoured terrain with multiple objects on it) it is important to provide: (a) objects of known size; and (b) sufficient detail on the surface of the ground (texturing or existence of small objects). The objects of known size tell the pilot how high the aircraft is and the textured surface conveys the shape of the terrain $[8,28]$.

Researchers at the University of Liverpool compared the effect of a high quality 
visual environment in a helicopter simulator with a degraded visual environment to examine the pilot behaviour when exposed to various scenery. The research concluded that pilots experienced higher workload in a low-quality visual environment in terms of compensatory control inputs to complete the same mission task as compared to operations in a good visual environment [29].

Furthermore, in another comprehensive study, specifically on FSTDs, researchers at the Embry-Riddle Aeronautical University tested ab-initio student pilots; teaching them in a combination of simulated and real airplane environments. They used the transfer of training (TER) technique to measure the effect of simulator training on pilot performance in ab-initio flight training sessions. Transfer of training can be calculated by the following equation:

$$
T E R=\frac{C-E}{E_{\mathrm{ftd}}}
$$

where $\mathrm{C}$ indicates the number of practice iterations an ab-initio student pilot performs to properly complete a manoeuvre in the actual aircraft, E corresponds to the number of practice iterations to accomplish the manoeuvre in aircraft after practicing in the FSTD, and $E_{\mathrm{ftd}}$ is the number of trials performed by the student in a simulated environment prior to the point at which E was measured. When learned skills have a positive effect on the development of a new skill it is known as positive transfer, while the hindrance of the acquisition of new skills is known as negative transfer. The results demonstrated that the practices in which the visual fidelity of the simulator is higher (with premiere 3D objects and artwork density in the virtual world), greater levels of transfer are achievable. [30]

Clinching the studies described above, in order to have a high-fidelity visual cueing system in an FSTD it is important to:

- Keep the frame rates of the rendering of the visual scene above $50 \mathrm{~Hz}$ in order 
to experience a smooth feeling of optical flow ${ }^{4}$ without jitteriness;

- Design the scenery as realistic as possible by using high-quality textures as well as spawning many $3 \mathrm{D}$ objects on the ground; and

- Increase the resolution of the screen and provide sufficient FOV to exceed the limitations of human vision.

\subsubsection{Motion and Vibration}

According to Transport Canada and the International Civil Aviation Organization (ICAO) manuals for flight simulators, having a motion platform is an asset in an FSTD, where FFSs are obligated to simulate motion $[2,31]$.

Motion has always been an interesting topic in flight simulation that has a major impact on the cost of simulators and associated training hours. Nevertheless, the need for motion cues in piloted flight simulation is still a widely debated issue [29].

In one study at MPL Simulator Solutions in Hong Kong, researchers investigated on a civil aircraft simulator (Boeing 747-400) with two groups of pilots. Without telling the pilots, the motion platform was off for one group. The results showed that there is no qualitative difference between groups and both related similar experiences of the training and evaluation; in particular, the group trained without motion said that their experience was similar to previous training in a FFS (with motion) and some of them did not mention motion at all. [32]

This has been correlated to a vast body of research having shown that accurate acceleration cues are not achievable currently, and that those available have not been proven to improve transfer of training between the simulator and airplane. Dynamic seats have been found to achieve the same transfer rate as motion platforms by

\footnotetext{
${ }^{4}$ Optical flow is the pattern of apparent motion of objects, surfaces, and edges in a visual scene caused by the relative motion between an observer and the scene.
} 
inducing limited acceleration and vibration to pilots where vibration inducers have been implemented under the pilot's seat. [33]

Vibration can increase the fidelity of a simulator through pulsations and stimulation of the vestibular system in various stages of flight such as engine start-up, taxing, take-off, touchdown, turbulence, flap deflection, etc.; however, it has not previously been recognized as a stand-alone cueing system.

\subsubsection{Sound in Flight Simulators}

The cockpit is a very noisy environment. Pilots hear a wide range of sounds from various sources. There are basically two types of sound that pilots must hear in order to have a realistic perception of sound:

1. Cockpit noise: such as airspeed or engine revolutions per minute (RPM), wind, sub-systems, ground rumble, etc.; and

2. Instrument alerts: such as warnings, navigation idents, radio chattering, etc.

In flight simulators, usually there are two modules generating each type of sound independently, one for instruments and alerts and one for the flight environment sounds such as engine noise and wind. The sounds are recorded by digital sound recorders for different manoeuvres and airspeed within the particular cockpit to be

analyzed; they are modified, and they are implemented in the simulator's sound generator modules. [1] 


\subsection{CURVS Project}

The Carleton University Redeveloped Vector Simulator (CURVS) is a collaborative project between Carleton University's Applied Dynamics Laboratory (ADL) and Aviation Training Resources (ATR) in Waterloo, Ontario. The goal is to revive and upgrade one of the old flight training devices that previously was used in flight training curriculum at the Waterloo Wellington Flight Centre (WWFC) named the 'VectorSim'. The VectorSim had hold a level 3 FSTD scale certification from Transport Canada.

Manufactured by Vector Training Systems in 2002, the VectorSim held a typespecific simulator for a Cessna 172 (C172) model with a poor and limited visual system that belonged to the previous generations of FSTDs.

Due to catastrophic software failure, the VectorSim was decommissioned and stored for a couple of years in Waterloo and then transported to Carleton University's ADL for assessment, research, and potential refurbishment. Based on recent experience and technologies developed within the $\mathrm{ADL}$ for building cost-effective flight simulators using COTS components that are highly reliable, the decision was made to upgrade the simulator to an advanced FSTD that is comparable to commerciallyavailable ones while attempting to exceed their performance at significantly lower cost.

\subsubsection{Objectives of the Project and the Thesis}

The main goal of the project was to implement techniques that were proven by previous 'KatanaSim' research in the ADL to design and build a robust cost-effective flight training device. The research-specific objectives of the project are subdivided bellow and the ones that are not entitled to this thesis are emphasized:

- Select a modern simulator software to be interfaced to all the instruments as 
the main image generator and flight model calculator software;

- Analyze and choose PC-based image generator hardware to achieve sufficient framerate (60 fps) as well as high-quality photo-realistic scenery and flight model with minimal cost;

- Design and build a wrap-around display with large FOV to provide a rear overshoulder view for the pilot;

- Implementation cost-effective COTS Short Throw (ST) projectors for a curved screen to be able to fit in a relatively small room;

- Carrying out calibration of the three projectors for smooth and perfect blending of the images using blending software technologies instead of expensive mechanical projection systems;

- Reverse engineer all the components including potentiometers, force-feedback motors, instruments, etc. of the VectorSim in an effort to find deficient parts to be fixed or alternatively replaced (not in this thesis);

- Develop software for controlling the flight instruments and engine gauges that is compatible with the new computer architecture (not in this thesis);

- Modify the cabin such as cutting expanded windows for an immersive FOV based on the new visual system;

- Attach seat vibrators to the crew seats to replicate vibrations experienced in a real Cessna 172 in different scenarios such as engine start-up, idle, taxiing, touchdown, flap deflections, etc. in an effort to increase the simulation fidelity;

- Perform flight tests to collect data as a source of reference data for comparison and tuning; 
- Tune the engine noise of the C172 based on real aircraft recorded sound files;

- Carry out qualitative and quantitative experiments on the simulator to determine its fidelity.

The funding for this collaborative project was provided by Connect Canada, the Ontario Centre of Excellence (OCE), and Aviation Training Resources (ATR) in Waterloo, Ontario, Canada. Three graduate students have contributed to the project; and each accomplished a portion of the tasks. The author developed the visual cueing, audio cueing, and vibratory cueing systems for the CURVS project, as well as supporting some of the other tasks.

\subsection{Outline and Thesis Overview}

This thesis is based on the redevelopment of a flight training device rebranded as CURVS that was formerly used at the WWFC as a C172 level 3 FSTD, certified by Transport Canada. While the mechanical components were well maintained, the main software of the FSTD was malfunctioning, hence causing the FSTD to be dysfunctional. The research is built upon reverse engineering of all the components of the FSTD including the instrument panel devices, control units, and force feedback system; as well as upgrading the visual system, audio system, computers, software, etc., and also adding a vibratory cueing system to the unit.

In Chapter 2, the design and construction of a curved projection system with 220 degrees horizontal FOV (HFOV) using inexpensive COTS components is explained. As the projectors are designed for single flat screens, their images needed to be blended and transformed to achieve an immersive image, and calibration was also performed. Moreover, the scenery of the Waterloo airport and its surrounding area was another major design task for the visual cueing that will be described in Chapter 2 . 
Chapter 3 describes the development of vibratory and sound cueing systems for the CURVS project. By utilizing the low-frequency audio signals feeding through the transducers, seat shakers generate vibrations which in turn enhance the fidelity of the simulation. Also, the implementation of the sound system as well as tuning the engine noise using recorded sound files from the aircraft is explained.

Chapter 4 describes the flight tests that were performed for quantitative data collection (acceleration data, field of view analysis, sound recording, sound pressure level, and light intensity) as well as the subsequent PSD analysis of vibration and sound data. Moreover, a qualitative evaluation test that was carried out during the CURVS project is described and discussed in Chapter 4.

Lastly, Chapter 5 presents the conclusion of the research, contributions and potential future research opportunities. 


\section{Chapter 2}

\section{Visual Cueing System}

In this chapter, a visual cueing system (VCS) for FSTDs is defined and its requirements for certification are outlined. The specifications and procedures for the CURVS VCS, in terms of design, analysis, build, and tuning, are addressed.

\subsection{Main Aspects of a VCS}

A high-quality VCS in a flight simulator should satisfy multiple requirements to be eligible for certification from Transport Canada or any other regulatory agency. Also, to provide a realistic feel of flying it is necessary to provide sufficient FOV, complex 3D scenery that runs smoothly without noticeable latency or jitteriness, and objects having accurate size and shape that appear in the virtual world with adequate resolution for human eyes. Taking all these factors into account is important as they dictate the total cost of the VCS. In this section, all these factors are considered. 


\subsubsection{FOV Versus Screen Resolution}

\section{FOV}

FOV is generally shown by an angle in degrees which defines the maximum extent that human eyes can observe in an specific environment. It could be defined both horizontally (HFOV) or vertically (VFOV). In flight simulation, the best solution to provide sufficient FOV both horizontally and vertically is to implement a curved screen. Some simulators utilize a cylindrical screen which is laterally curved but vertically flat. Cylindrical screens are the most common type of screens that are utilized in high-fidelity FSTDs since they are easier and cheaper to build than spherical screens and provide greater immersiveness than single or compound flat screens. Other simulators, however, such as FFSs implement double curved screens (DCS) that result in a more realistic perception of depth. Also, other types of simulators, such as fighter jet or helicopter simulators, often utilize dome-shaped screens as they need more VFOV than their counterparts.

\section{Resolution}

Usually resolution is measured as the number of pixels per inch (PPI) projection screen. A 300 PPI device (it could be any type of screen), for instance, projects 300 pixels in every direction along its screen. More pixels is usually associated with a higher-resolution image. For example, Figure 2.1 compares the pixel density of an image on two different screens with various PPIs. As the number of pixels increases more computation power is required to render each frame which in turn adds to the cost of the simulator. Moreover, the acuity of the image changes by the distance of the observer from the screen. The further away the observer is from the screen, the smaller the screen, and correspondingly the smaller each individual pixel is, and 
thus a better resolution is achieved. Therefore, there is a need for better defining the resolution that is also correlated to the distance of the observer from the screen.
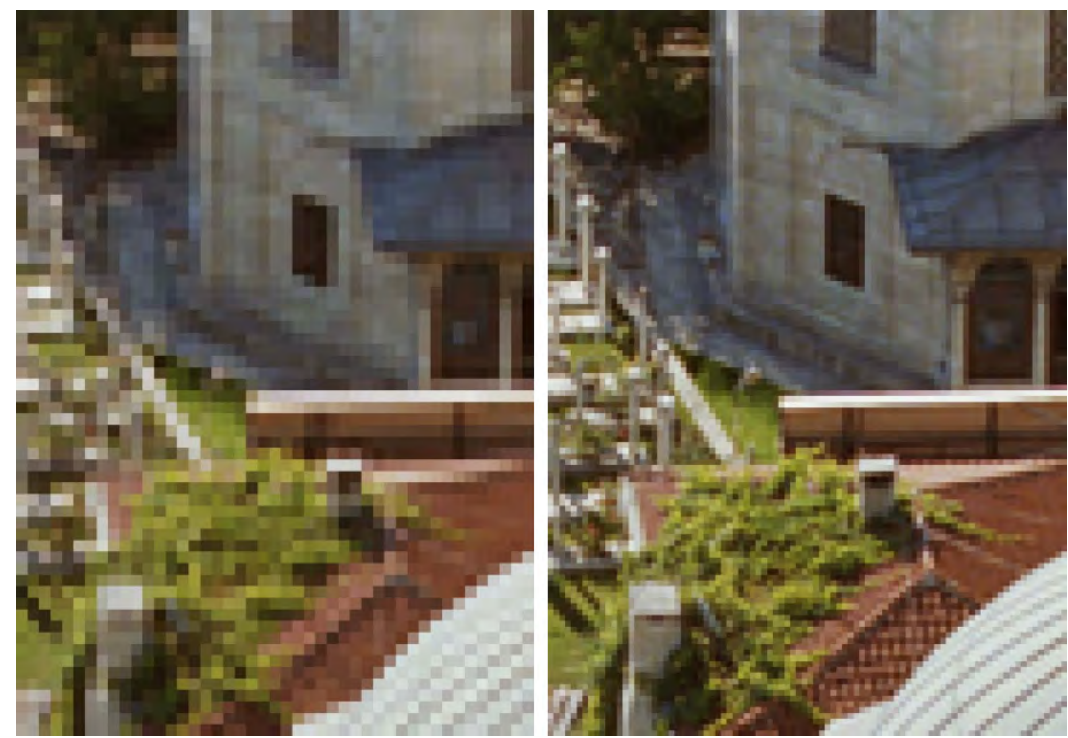

Figure 2.1: PPI comparison: Full HD image on the left and $4 \mathrm{~K}$ image on the right.

The resolution of the human eye is measured as the acuity that one can perceive. It is measured in arc minutes (arcmin) where every arcmin represents 1/60 of a degree. Since the resolution is measured as an angle, it can be translated into a separation in, say, inches at a certain distance. A 1-foot $(0.3 \mathrm{~m})$ ruler at a distance of about 57 feet $(17.4 \mathrm{~m})$ would appear to be 1 degree across (about twice the size of the full Moon). Therefore, if human eyes had a resolution of 1 degree, then the ruler would just appear to the eyes as a dot, as illustrated in Figure 2.2. Also, higher resolution means smaller arcmin.

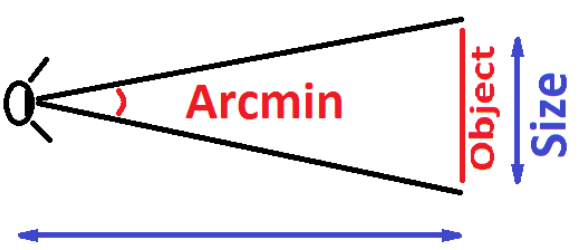

Distant

Figure 2.2: Arcmin is an angle by which the human eye's resolution is defined. 
In flight simulation, as the pilot-eye-point (PEP) is nearly constant, it is possible to define the absolute resolution for the screen. In order to obtain a high-resolution image, the absolute resolution should be around 1 arcmin for every individual pixel as this is the average resolution of human eyes [1].

One way to increase the screen resolution is to place the projectors closer to the screen. The closer the projectors are to the screen, the smaller the image; and also the narrower each individual pixel will be. As such, smaller arcmin could be attained. Although a better resolution could be obtained this way, as the size of each image becomes smaller the total FOV diminishes both laterally and vertically. Thus, there exists a tradeoff between the desired FOV, distance from the screen, and the computation power required to achieve a reasonable resolution.

\subsubsection{Parallax and Collimation}

The parallax effect, according to the Oxford Dictionary, is a displacement or difference in the apparent position of an object viewed along two different lines of sight, and is measured by the angle or semi-angle of inclination between those two lines [34,35]. Parallax is a natural phenomenon caused by various distances of different objects in front of an observer, such that the distant object appears to move slower than the closer one having the same speed. Figure 2.3 illustrates this phenomenon. It has an application in astronomy by which it becomes possible to measure the distances of the stars and galaxies from the earth. In flight simulation, however, it is indeed a negative effect. As the screen is in close proximity (within 2-3 meters away) of the PEP, looking at the same object from different locations on the flight deck (pilot and co-pilot seats) shifts the location of the object. In other words, the pilot and co-pilot see the same object (e.g., the runway) in different locations. This is not realistic; as in the real aircraft, the out-the-windows (OTW) scene can be up to hundreds of meters away from the PEP. Moreover, if the focus of the scene is at the centre of the screen 
the pilot feels that the aircraft is yawing to the left. This is illustrated in Figure 2.45.

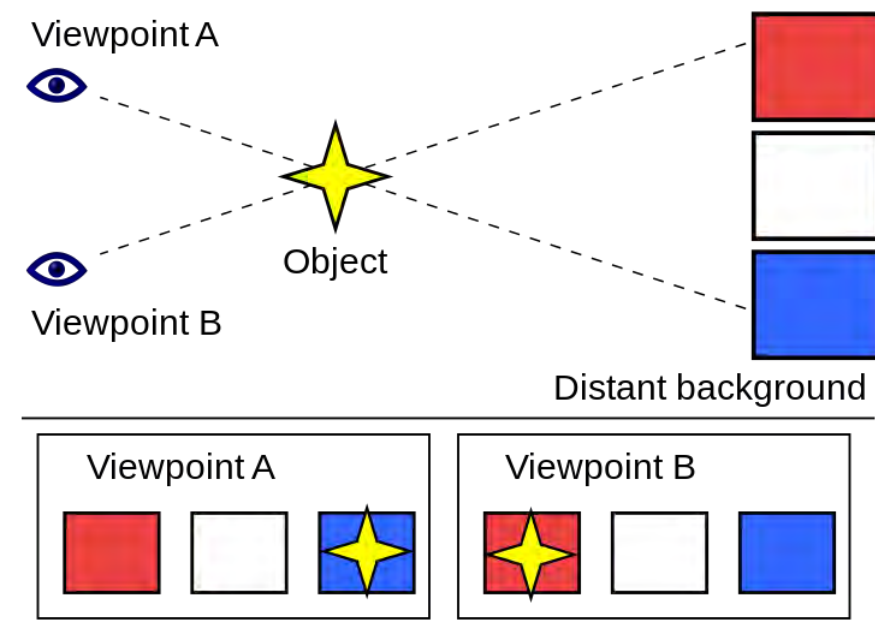

Figure 2.3: Parallax effect. Different POVs see different objects.

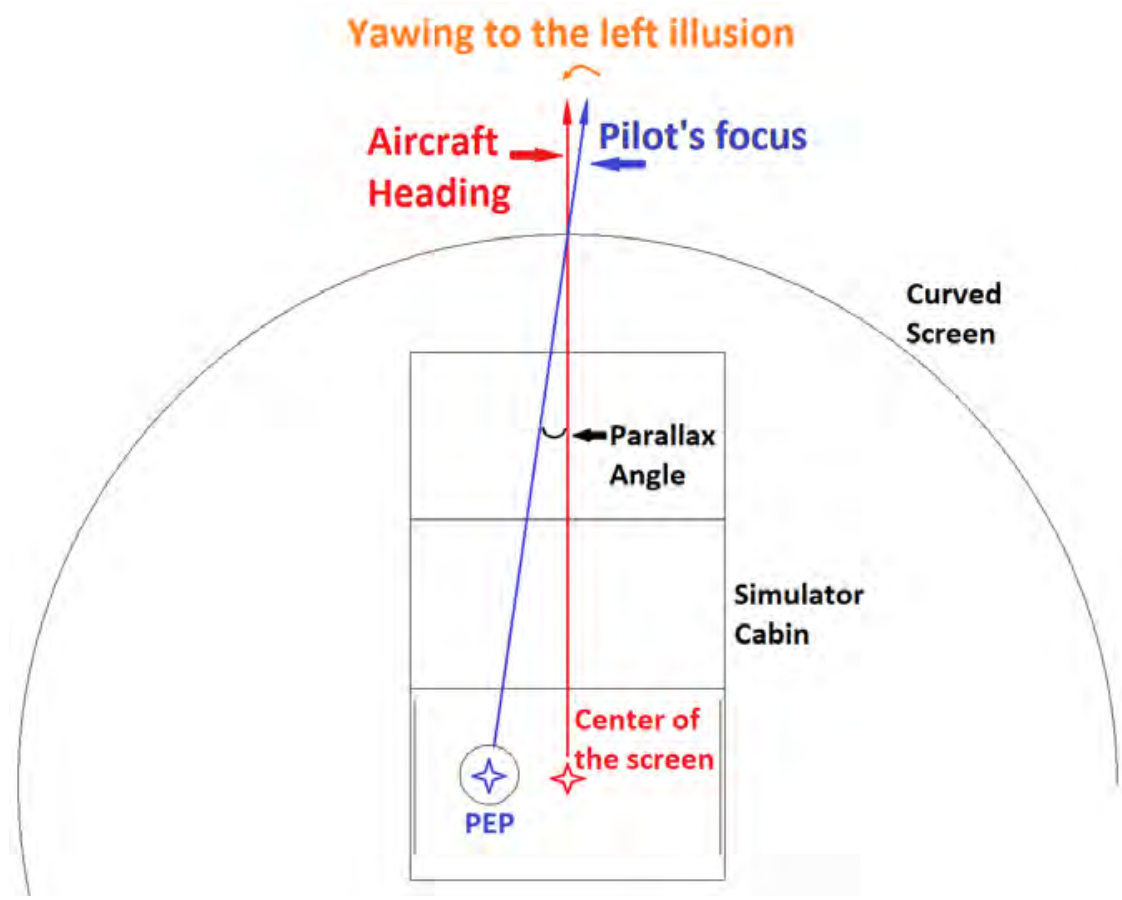

Figure 2.4: Parallax effect. When the scene is focused at the centre of the screen, the pilot feels that the aircraft is yawing to the left.

Modern flight simulators have alleviated this problem by taking advantage of collimation mirrors, pushing the reflected image further back from the PEP to diminish 
the parallax angle. Instead of looking directly at the screen, the pilot looks into a curved mirror that reflects the image thrown from the screen above the mirror. This causes the so called cross-cockpit view of the OTW scene in both pilot seats. One drawback of the collimated displays is that the mirrors are expensive to build. They are usually made of mylar fabric coated over a vacuum box that is connected to a vacuum pump. As the air inside the box is evacuated the pressure difference between the outside and the inside of the box results in a smooth and wrinkleless curved mirror surface. This is most useful for airline pilot training for which both the pilot and the first officer are responsible for controlling the aircraft [1]. FSTDs are mainly used to teach the student pilots to fly the aircraft from the pilot seat side; thus, there is no need for a cross-cockpit view. As such, by only focusing the centre of the screen to the captain seat it is possible to mitigate the parallax effect.

It is also important to note that based on the Manual of Criteria for the Qualification of Flight Simulation Training Devices [31], for an FSTD, the only criterion for certification is that the parallax effect should be less than 10 degrees and also the simulator must have the option to switch the focus of the scene back and forth between pilot and co-pilot PEPs.

\subsubsection{Scenery Versus Frame Rate}

As discussed in Chapter 1, the amount of scenery content impacts the simulation fidelity. However, as the density of the scenery content increases, meaning more 3D objects and higher resolution textures and terrain data, more computation power is needed to render the content in real-time.

In real life, for humans, everything seems to be continuous and instantaneous. Humans perceive every motion in everyday life smoothly. A computer, however, calculates the program following a step by step processing of instructions. Each of these instructions takes a finite number of machine cycles and each of these cycles is 
clocked at the speed of the processor. In order to render a smooth and continuous stream of images, like televisions that are broadcast at 25 frames per second (meaning that every second 25 different images are shown), in flight simulation three criteria have to be satisfied [1]:

1. At least 50 cycles per second should be calculated to send 50 FPS to the visual system.

2. Every frame should be calculated within the FPS time limit (20 milliseconds in a 50 FPS setup), as demonstrated in Figure 2.5.

3. The number of FPS should match with the projector's refresh rate to avoid disparity between calculated frames and the projector's image generation frequency.

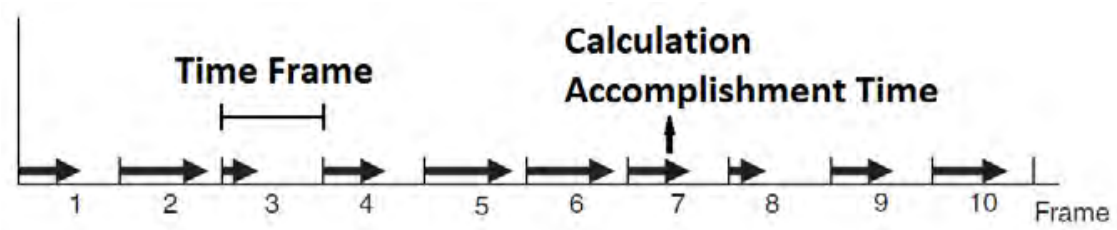

Figure 2.5: Real-time frames. Calculations must be done within the time limit.

The first two criteria are a matter of hardware and software configuration (image generator). The third is a matter of COTS-available projectors; where today's projectors are set to $60 \mathrm{~Hz}$. Therefore, to experience a smooth real-time simulation, the image generator must currently be able to calculate at 60 FPS to match the refresh rate of the projectors.

\subsection{CURVS Visual Cueing System}

During the development of the CURVS VCS all the above discussions have been taken into consideration. To explain the design, analysis, and build of the VCS, step by 
step, all the procedures are divided into three sections: Pre-design Procedures, Image Generator, and Projection System.

Before getting into detail in each of these steps, important features of the CURVS VCS that are inspired by investigations early in the project are explained.

\subsubsection{Pre-design Procedures}

During the preliminary stages of the research and prior to designing the projection system, a pilot instructor at Rockcliffe Flying Club in Ottawa was interviewed. The goal was to exploit useful information of the most prominent manoeuvres that are performed at the ab-initio stage of pilot training. The information learned in the interview was also reviewed and practiced during the flight tests that are investigated in detail in Chapter 5.

It became clear after the interview that during ab-initio flight training, pilots learn five main manoeuvres: 1) taxing, 2) takeoff, 3) cruise flying, 4) traffic circuits, and 5) landing. It is obvious that the more immersive the visual system is, the more realistic the simulated flight experience feels. Nonetheless, greater FOV requires more projectors and a larger screen and that adds to the total cost of the VCS.

After the interview and performing the flight tests, cruise flying, landing, and circuit manoeuvres were found to be more dependent on visuals than the others; hence, their procedures were investigated to achieve the required FOV for the simulator. During takeoff, the pilot looks over the runway. For this, not much FOV, both vertically and horizontally, are required to accomplish this task. Therefore, only flying level, landing, and circuit manoeuvres are used to determine the required FOV for the VCS. The results are summarized as follow: 


\section{- Flying Level}

The first skill that pilots learn during ab-initio training is the ability to fly the aircraft level which is necessary for cruise flying. The pilot has to be able to keep the airspeed, altitude, and the attitude of the aircraft at a desired level for cruise flying. The pilot is also required to concentrate on the yoke, throttle, rudder pedals, and the trim wheel to keep the plane level while keeping track of the main six pack instruments (attitude indicator (AI), altimeter, airspeed indicator (ASI), vertical speed indicator (VSI), turn coordinator (TC), and heading indicator $(\mathrm{HI}))$. In the case of ab-initio training, pilots also have to know how to fly the aircraft visually which is a practice to avoid the dependency of the pilot on instruments.

In order to keep the aircraft level in a Cessna 172 it is important to see $1 / 3$ ground and $2 / 3$ sky through the front window and try to keep this pattern to fly level. Figure 2.6 shows how it should look in a Cessna 172 during cruise flying. This rule is implemented in the CURVS VCS carefully to make sure that pilots can practice the cruise flying manoeuvre using the simulator. It also helped to have a rationale to calculate the required vertical field of view (VFOV) for the simulator.

\section{- Landing}

Landing an aircraft safely is also an important learning objective for ab-inito pilots. While trying to land, pilots constantly look at the runway to centre the aircraft along the runway as well as following the glide slope with the appropriate airspeed. To do so, the geometry, shape, and the size of the runway that appear in front of the pilot, as well as the existence of 3D objects having accurate size and shape that help to estimate the altitude are critical. Moreover, a precision approach path indicator (PAPI) or on some runways a visual approach slope 


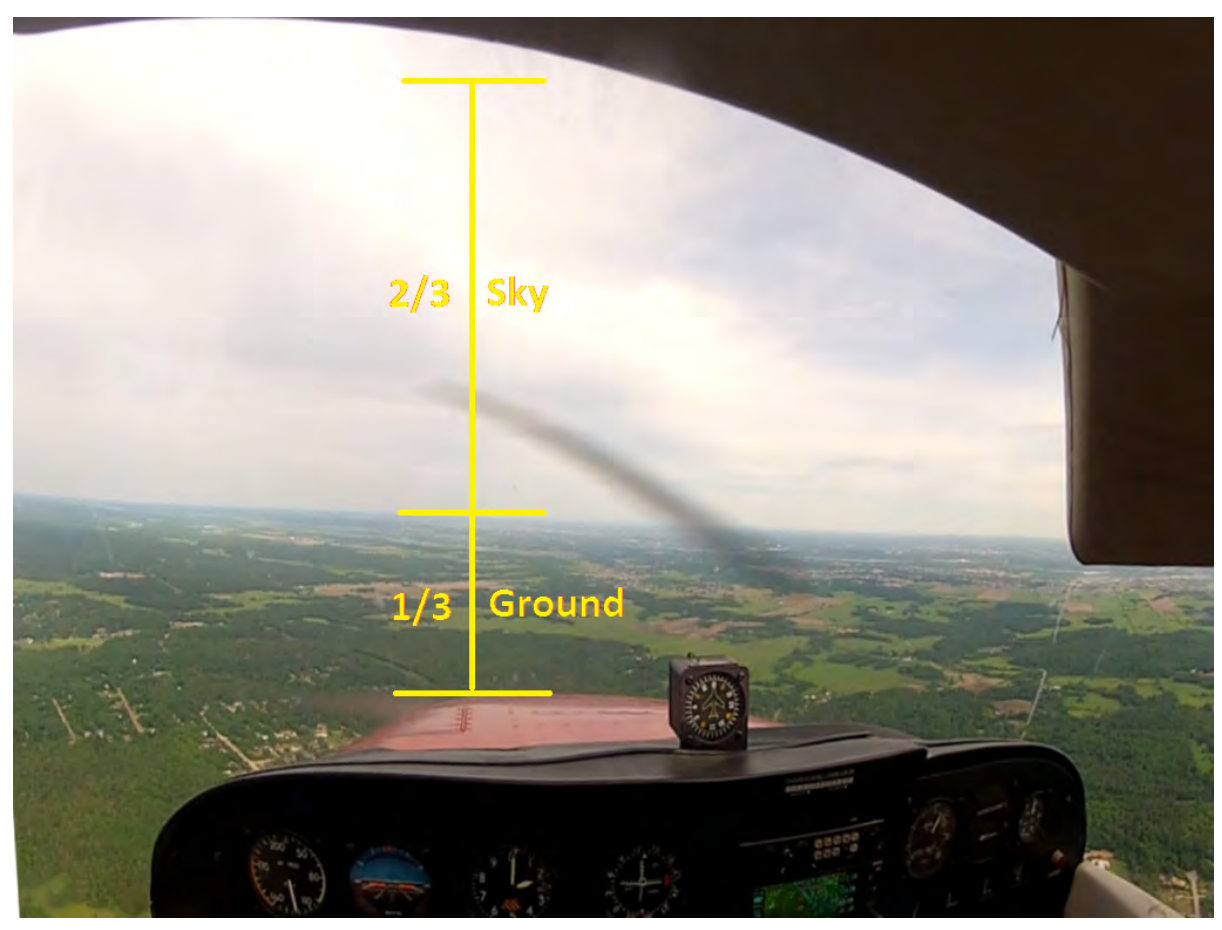

Figure 2.6: Panaromic view of the front window in a Cessna 172 during cruise flying.

indicator (VASI), as well as approach lights that help the pilot to stay within the predefined glide slope pattern are crucial. The other vital factor, while landing, is the existence of a windsock next to the runway so that pilot can perceive the wind's direction and speed. Figure 2.7 illustrates the final stage of landing where the pilot is looking at the runway to correct the aircraft attitude and altitude to stay centred and on the glide slope of the runway. 


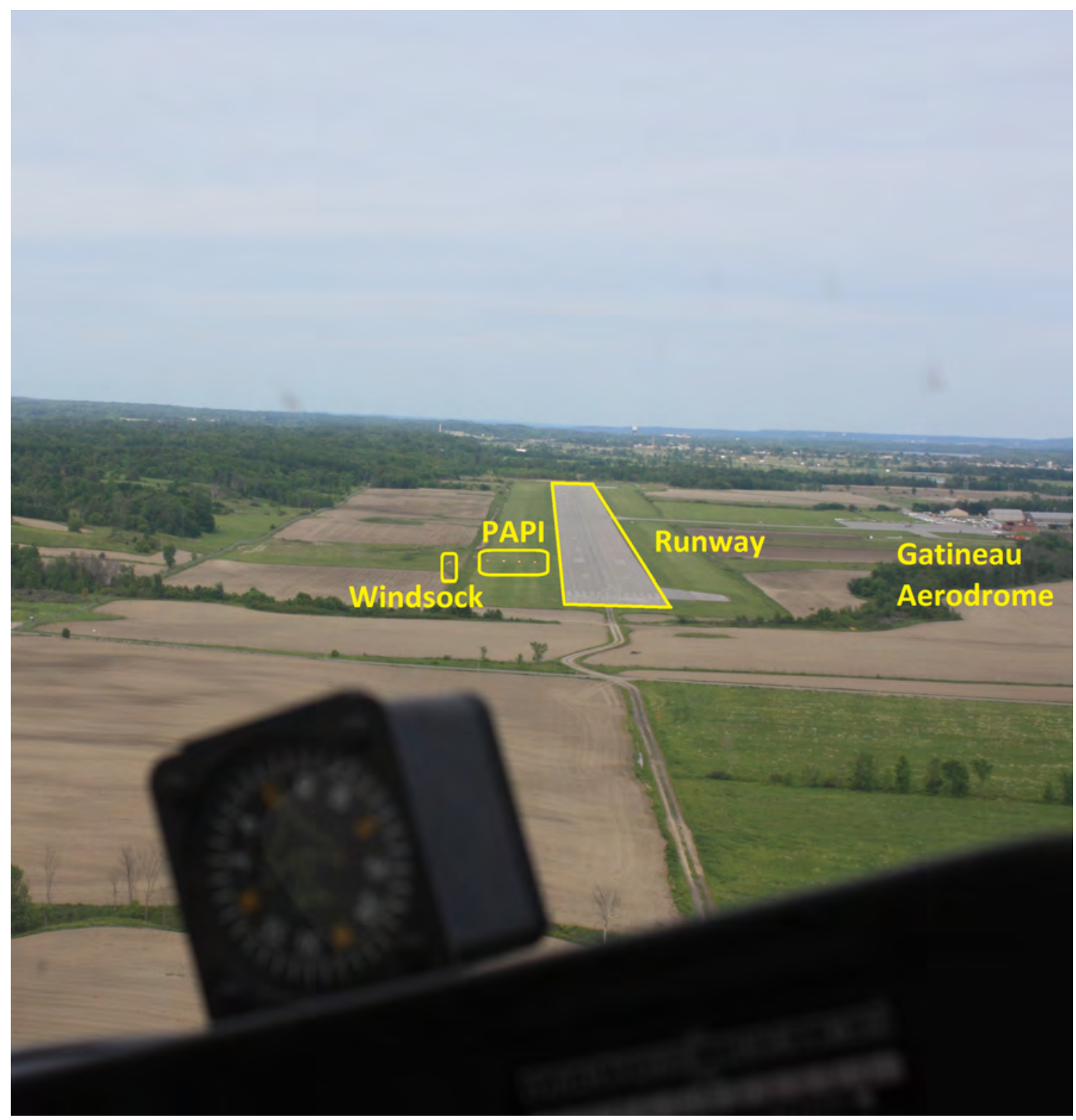

Figure 2.7: Touch-and-go with the Cessna 172 during flight tests in the Gatineau aerodrome. 
- Circuit patterns:

Correct execution of circuit patterns is one of the preliminary steps for obtaining a PPL for pilots. Circuits are the standard manoeuvre every pilot has to follow when deciding to land at an airport. The standard altitude for circuit patterns is usually 1000 feet above the airport's elevation and also most of the circuit patterns are left-handed (LH). It is simply because the pilot's seat is located on the left side of the cockpit thereby providing more FOV to the left than to the right side of the aircraft. The pattern consists of five major legs named: a) takeoff (or departure), b) crosswind, c) downwind, d) base, and e) final. During the takeoff, the pilot follows the standard takeoff procedures until reaching a certain altitude and airspeed (depending on aircraft type) at which point it is time to make a 90 degree left hand turn to the crosswind leg and then to downwind. In the downwind leg, the pilot tries to fly parallel to the runway while keeping the altitude about $1000 \mathrm{ft}$ above the airport. At an appropriate time, the aircraft must turn left to the base leg and start descending. Once on the base leg, the pilot has to keep his eyes on the position of the aircraft relative to the runway to decide when to make the last turn onto the final leg. Figure 2.8 illustrates a standard circuit pattern and Figure 2.9 shows the pilot view in a Cessna 172 on the downwind leg looking to the left parallel to the runway.

From the pilot's perspective, there are no certain rules to follow to fly the circuit accurately. However, there are some suggested rules for pilots by which after practicing, flying circuits becomes easier. Apart from constantly watching the instruments such as the AI, altimeter, VSI, and HI, the pilot should use visual references to accomplish a successful circuit. In order to keep the aircraft parallel to the runway during the downwind leg, for instance, the pilot watches to the left side frequently. The tricky part for the pilot is to estimate when is 


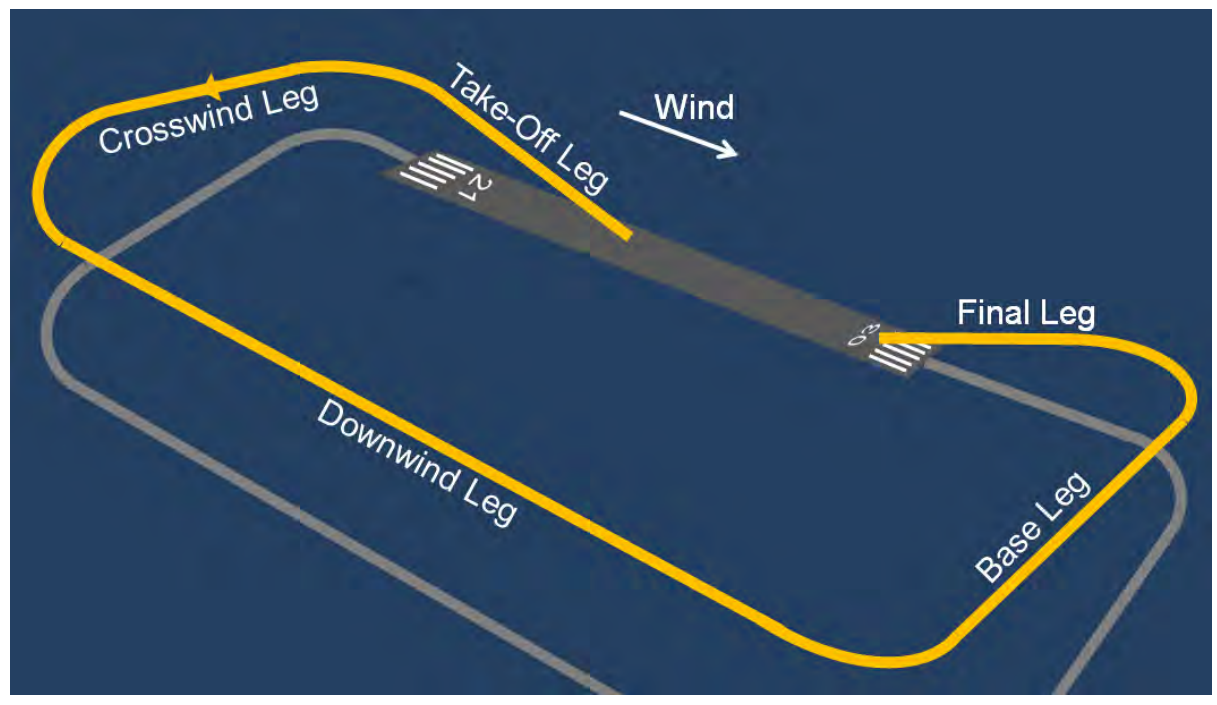

Figure 2.8: A standard circuit pattern chart.

the appropriate time to turn to the base leg from downwind. The convention in a Cessna 172 is that the pilot shall look over his or her shoulder to the left about 45 degrees to see the end of the runway. Once the end of the runway can be seen from that angle it is time to do the 90 degree left turn to the base leg. This practice is called over shoulder view (OSV) and Figure 2.10 reveals this trick from the actual Cessna 172 experiment doing a circuit.

This practice helped to decide that the simulator needs more than 180 horizontal FOV (HFOV) in an asymmetric way providing extra visuals on the left-hand side (LHS) of the simulator. By having more FOV on the left side, the pilot is able to accomplish a complete circuit using only visual references in the simulator like in the actual aircraft. This feature has been implemented in the CURVS VCS that will be discussed later.

\subsubsection{The Image Generator}

In flight simulation, the combination of customized software and hardware as a unit is called the Image Generator. There are multiple companies in the industry, such 


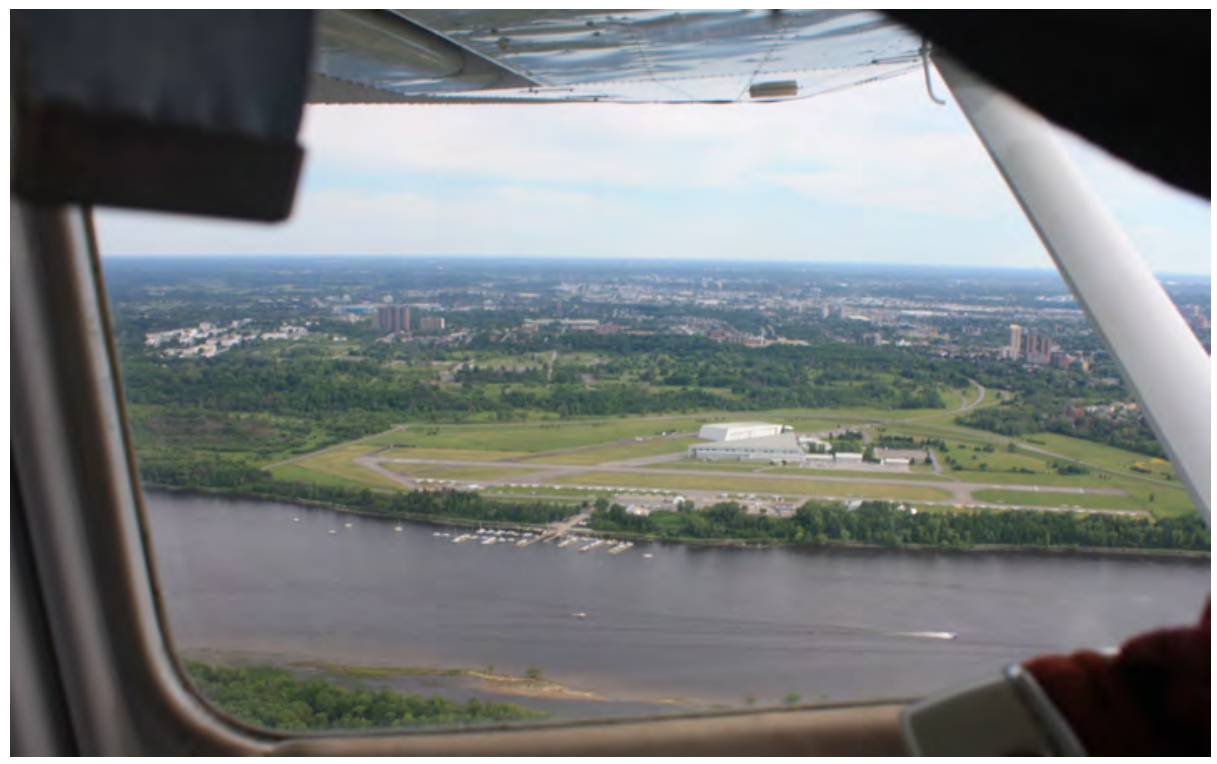

Figure 2.9: Cessna 172 flying in downwind leg at the Rockcliffe aerodrome.

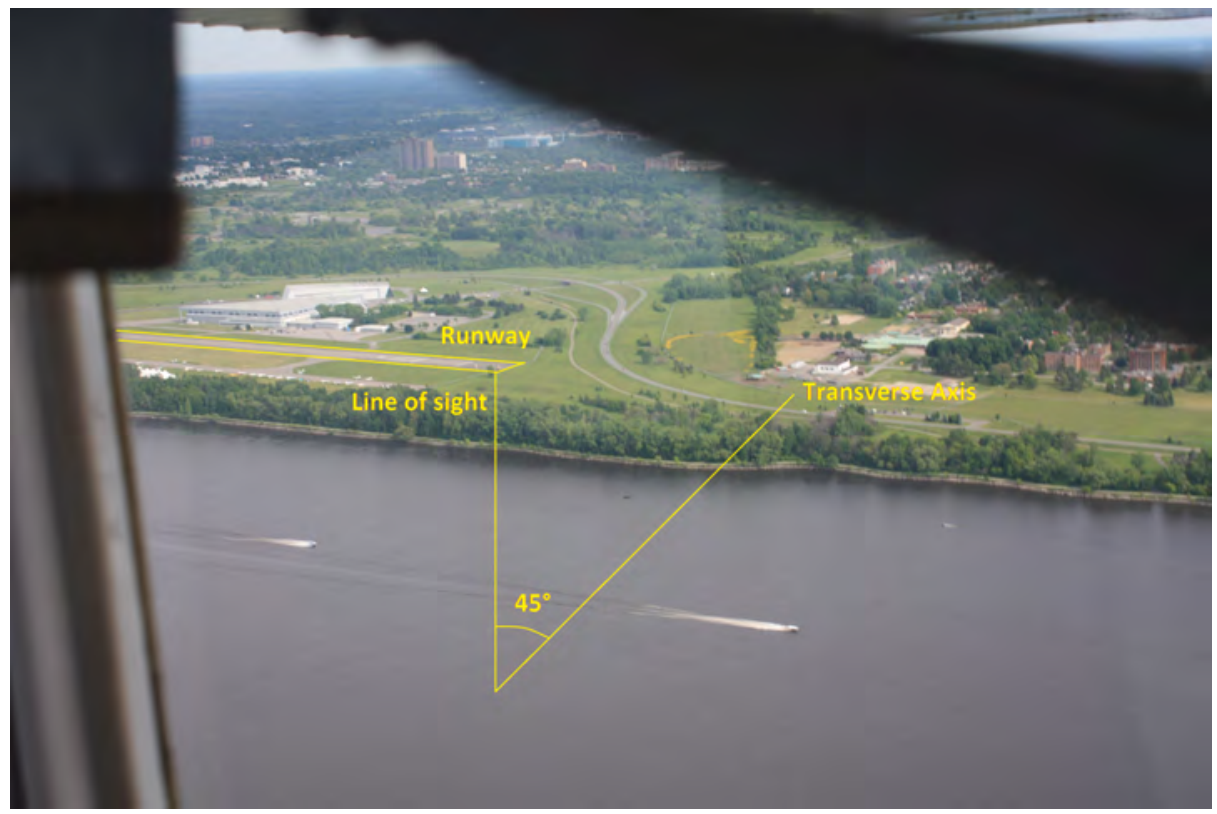

Figure 2.10: OSV in a Cessna 172 flying on the downwind leg ready to turn to base leg at the Rockcliffe aerodrome.

as Rockwell Collins that produces image generators with a range of capabilities and prices [36]. An image generator is made of two components: a) Hardware, b) Software that are discussed below. 


\section{Hardware}

The image generator of the CURVS project uses four computers, all of which are running the main software that is responsible for different tasks. Basically, there is a main computer and three slaved channels for image generation (visual channels).

The main computer that is directly connected to the instructor operating station (IOS) shows many options to the instructor such as a moving map, flight tracker, time, and weather and also has access to buttons to implement preplanned failures during the flight. Moreover, the instructor station computer calculates the flight model as an output of the control inputs.

The other three computers are connected to projectors to provide the visual system. The hardware specification of these computers is important since the goal is to reach 60 FPS to avoid excessive latency. However, the more computation power, the more cost will be associated with the image generator. Therefore, it is critical to find the most cost-effective hardware available in the market that is compatible with the software.

Visual channels need more graphical power than the instructor station computer, hence having a state-of-the-art graphical processing unit (GPU) is not necessary for the IOS to avoid latency. Nevertheless, in the CURVS project as the selected software has the capability to generate the same scenery as visual channels through the IOS computer, the same GPU was selected for the IOS as well. This is beneficial for the pilot instructor to see how the flight manoeuvres are performed by the student without the need to look at the curved screen.

The visual system diagram is shown in Figure 2.11. The list of specifications for the computers as well as a complete hardware diagram of the CURVS simulator, including all the connections to the instruments, can be found in Appendix A. 


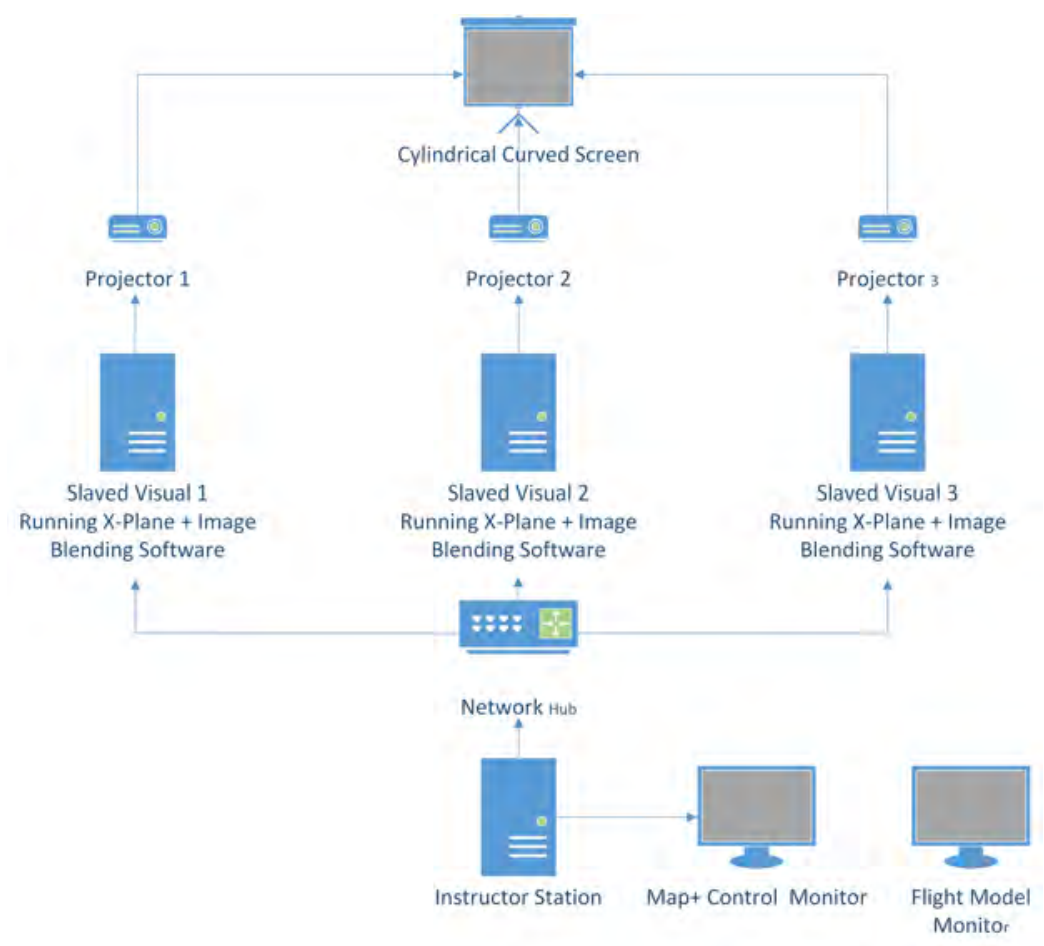

Figure 2.11: CURVS visual system diagram.

\section{Software}

Nowadays, there are two COTS simulation software package available in the market that many companies and flight simulator hobbyists use. For many years MSFS was the leading software in PC-based simulators; however, the project was shut down by Microsoft in 2009 [37]. Lockheed Martin bought the intellectual property of FS from Microsoft that lead to the announcement of a new product called Prepare3D [38]. On the other hand, Laminar Research that had already released their new flight simulator software called X-Plane before Prepare3D a few years back, was becoming popular [39]. Figure 2.12 shows X-Plane's C172 flying.

Like Microsoft Flight Simulator, Prepare3D is targeting the gaming market where X-Plane's focus is more on the engineering aspects of a flight simulator. After investigating the existing software in the market as the primary simulation software for the CURVS project, between Prepare3D and X-Plane, X-Plane was deemed preferable 
for the following reasons.

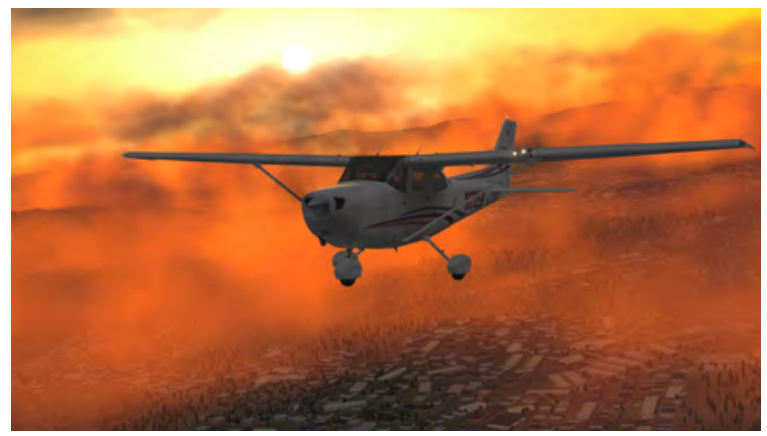

Figure 2.12: A Cessna 172 flying in a sunset scenario in X-Plane 10.

- It generates a more accurate flight model (by using Blade Element Theory) in comparison to look-up tables used by Prepare3D.

- It is easy to establish communications through the User Datagram Protocol (UDP) network for data transfer between computers and instruments.

- Good support is available from the producer as well as third party online communities.

- It is well documented and easy to understand procedures to design customized add-ons such as aircraft, scenery, and plugins.

- There exists, easy-to-use (ETU) software that comes with the simulator for modifying aircraft and scenery.

- It is compatible with off-the-shelf CAD software such as Blender, SketchUP, 3D Max, etc.

- It provides the option of recording simulated flight data for subsequent data analysis. 


\subsubsection{Projection System}

\section{The Design}

One major cost of simulators is the building in which it is assembled. Therefore, before starting to design the CURVS screen, it was important to know how much space is available for the simulator in the facility in which it will ultimately be installed. There were two available rooms in the WWFC main office building. One has a short ceiling; and CAD design revealed that a VCS in this room would cause shadows of the cabin to appear on the screen. The alternative room that was larger, was not serviced by the air conditioning system and this would add to the cost of the project. Finally, as the WWFC was renovating one of the floors in their building, they decided to make a sufficiently large room for the CURVS. It has $20 \times 20 \mathrm{ft}(6.1 \mathrm{~m})$ of floor area and a ceiling more than $12 \mathrm{ft}(3.7 \mathrm{~m})$ high which was found to be adequate for installing the CURVS.

Furthermore, the size of a curved screen is also limited by the projector's image size. Larger images result from greater distance between the projector and screen. This problem was solved by choosing ST projectors that in comparison to regular projectors project a bigger image within the same distance from the screen. This feature is measured by the so-called throw-ratio (TR). Throw ratio can be calculated as:

$$
T R=\frac{W}{D}
$$

where $\mathrm{W}$ is the width of the image and $\mathrm{D}$ is the distance of the projector from the screen, see Figure 2.13. By this definition, the smaller the throw ratio the bigger the image assuming a constant distance. Also it is useful to define throw angles for the projected image (both laterally and vertically) as follow:

$$
\alpha=\arctan \left(\frac{W}{2 D}\right) \quad\left[^{\circ}\right]
$$




$$
\psi=\arctan \left(\frac{H}{D}\right) \quad\left[{ }^{\circ}\right]
$$

where $\alpha$ and $\psi$ are horizontal and vertical throw angles, respectively, as demonstrated in Figure 2.14.

The projectors that have been chosen for the CURVS visual system are HT1085ST BenQ Full-HD $(1920 \times 1080$ pixels $)$ model, where ST stands for short-throw [40]. This model and brand was found to be used by many flight simulator hobbyists since it is an affordable COTS products with Full-HD resolution. The specifications of the projector can be found in Appendix A.

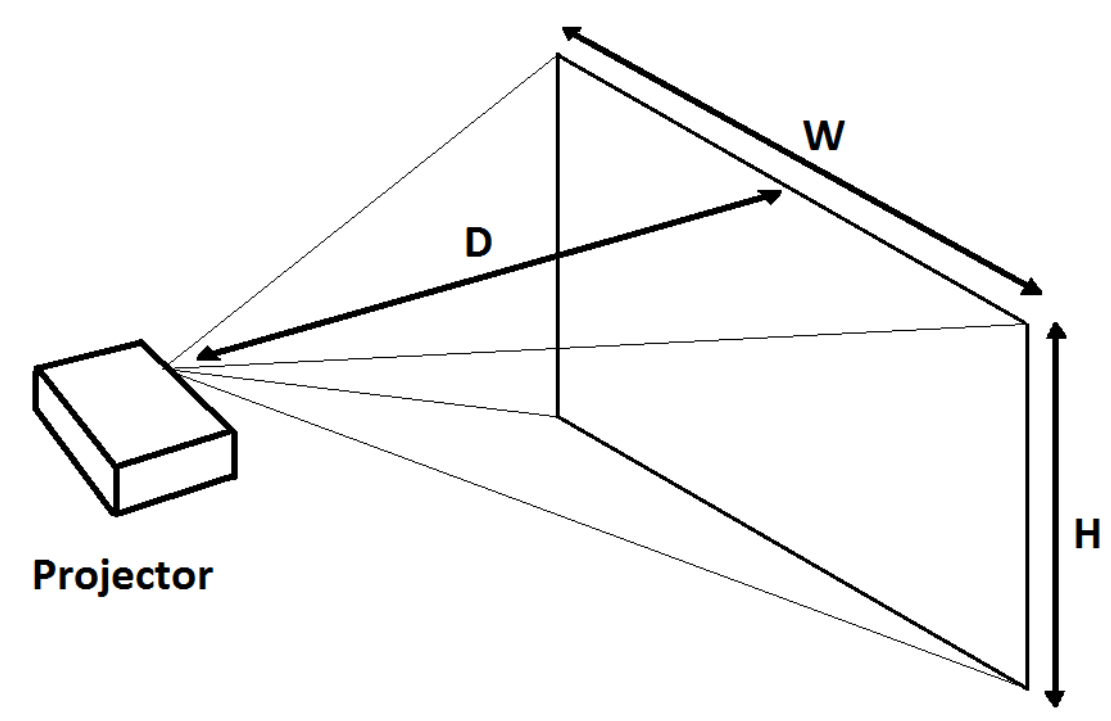

Figure 2.13: Projector's image geometric parameters.

As the projector has physical zooming capability, both $\alpha$ and $\psi$ could be changed accordingly. Therefore, the limits of $\alpha$ and $\psi$ are measured by measuring $\mathrm{D}, \mathrm{W}$, and $\mathrm{H}$ while the image was projected onto a flat wall, and using Equations 2.2 and 2.3. 


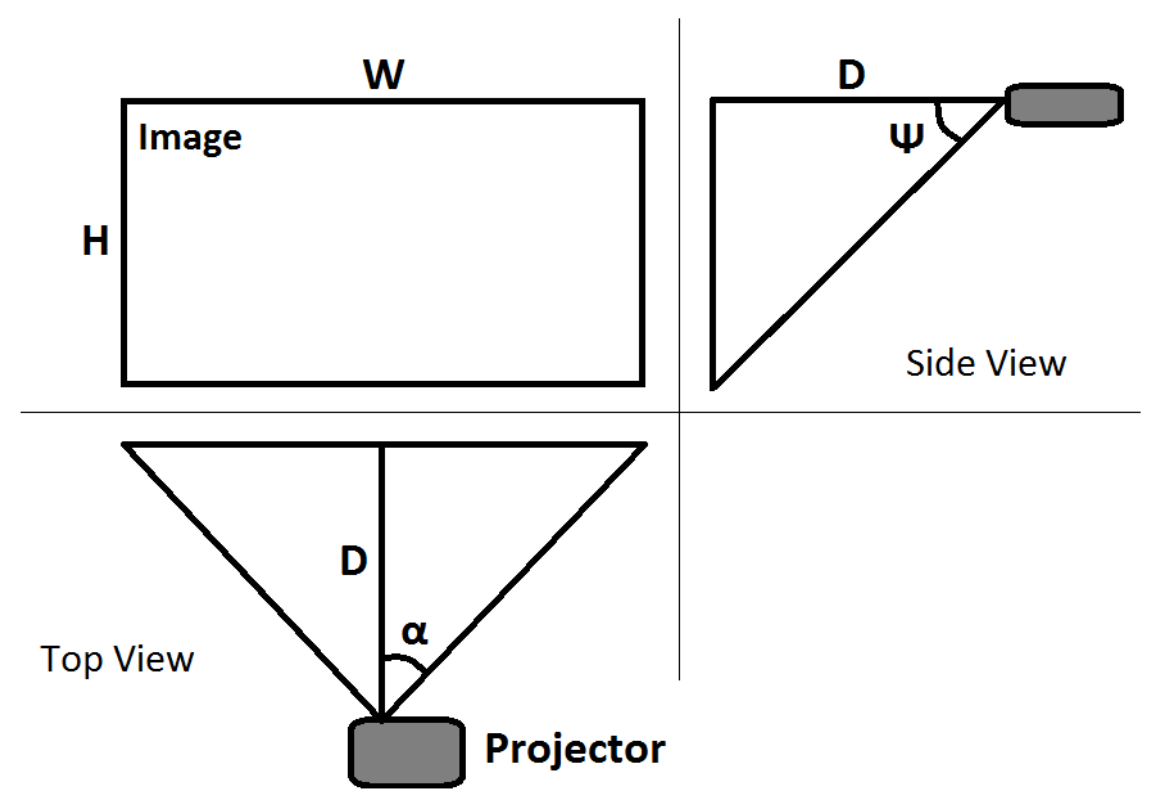

Figure 2.14: A projector's throw angles.

The result was found to be:

$$
\left\{\begin{array}{l}
31.0<\alpha<35.1 \\
34.5<\psi<40.7
\end{array}\left[^{\circ}\right]\right.
$$

In order to find a correlation between the HFOV (desired) and the needed screen radius to achieve it, geometric analysis was performed. First it is important to note that three projectors were decided upon. Second, according to the manual of the blending software, that will be discussed in the next section, the images of the projectors have to be overlapped about 10\%. In Figure 2.15, all the calculation parameters are illustrated, where $\mathrm{R}$ is the radius of the screen, $\mathrm{Y}$ is the distance of the projector from the centre of the screen, $\gamma$ and $\beta$ are the two angles as shown in the picture, and $\mathrm{X}$ is the width of the projected image onto the curved screen. $\mathrm{C}$ is also the total length of all the blended images onto the curved screen. X which is indeed an arc can 


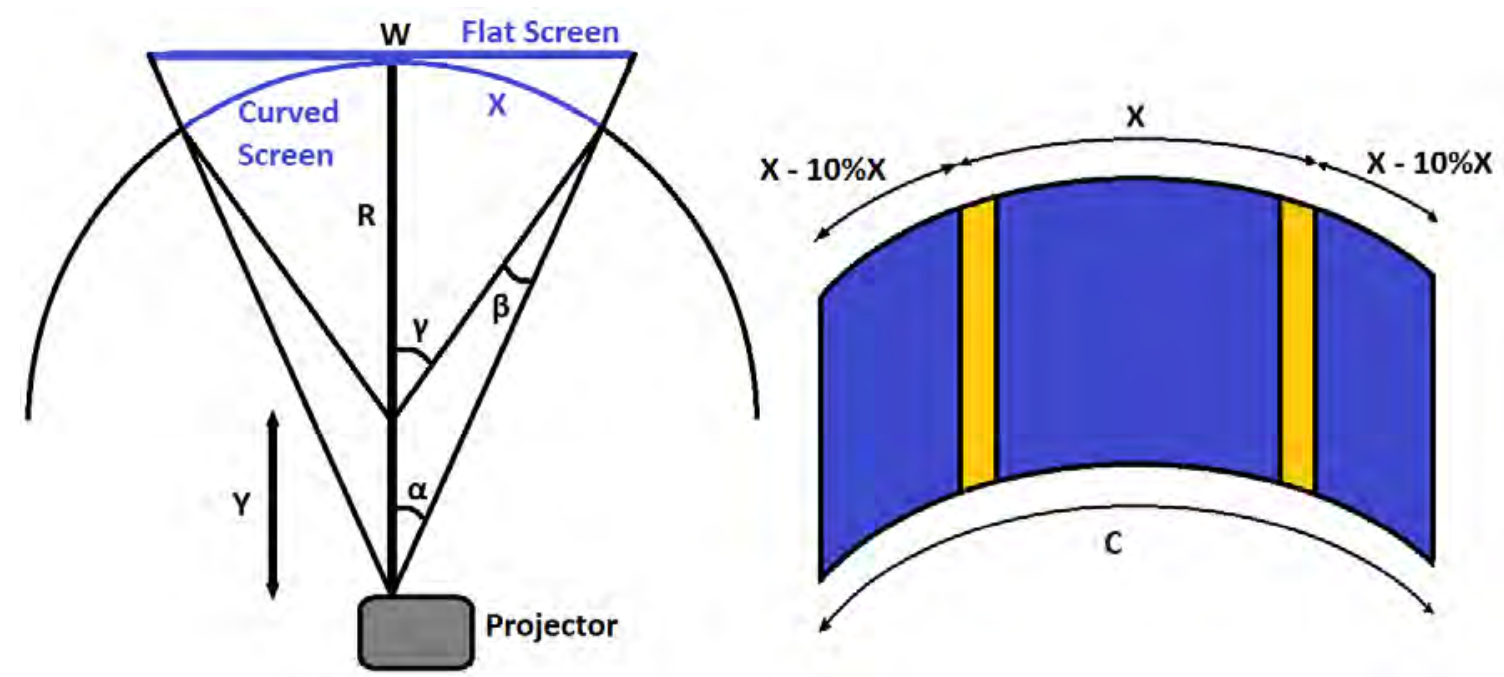

Figure 2.15: All the screen parameters with $10 \%$ overlap of the images.

be calculated as:

$$
X=\frac{2 \gamma}{360} 2 \pi R \quad[\mathrm{~m}]
$$

Also, $\mathrm{X}$ is correlated to $\mathrm{C}$ as shown on the right side of Figure 2.15:

$$
3 X-2(10 \% X)=C \rightarrow X=\frac{C}{2.8} \quad[\mathrm{~m}]
$$

$\mathrm{C}$ is also correlated to the total HFOV of the screen by:

$$
C=\frac{H F O V}{360} 2 \pi R \quad[\mathrm{~m}]
$$

By substituting Equation 2.5 into 2.6 and solving for $\mathrm{C}$ and then substituting the resulting $\mathrm{C}$ into Equation 2.7 and solving for $\gamma$ gives:

$$
\gamma=\frac{H F O V}{5.6} \quad\left[^{\circ}\right]
$$

According to the law of sines and triangle geometry it is obvious that: 


$$
\left\{\begin{aligned}
\frac{\sin (\beta)}{Y} & =\frac{\sin (\alpha)}{R} \\
\gamma & =\beta+\alpha
\end{aligned}\right.
$$

By substituting $\gamma$ into Equation 2.9 and solving for $\mathrm{Y}$, we obtain:

$$
Y=R \frac{\sin \left(\frac{H F O V}{5.6}-\alpha\right)}{\sin (\alpha)} \quad\left[{ }^{\circ}\right]
$$

Finally the distance of the projector from the screen, which is given by $D=R+Y$ becomes:

$$
D=R\left(1+\frac{\sin \frac{H F O V}{5.6}-\alpha}{\sin (\alpha)}\right) \quad[\mathrm{m}]
$$

Equation 2.11 means that given the radius of the screen and the desired HFOV and throw angle (zoom level), projectors have to be located at distance D from the screen.

Before designing the screen using CAD software it was also important to take into account the resolution of the image. Therefore, the projector's resolution was tested on a flat screen in a trial and error manner. The projector was moved back and forth on its average zoom level to find out the best distance from the screen for giving the best resolution possible while the image is adequately large. It was found that if the projector were placed about $2.6 \mathrm{~m}$ away from the screen and the pilot sits about the same distance from the screen, the resolution appears to be sufficiently high with no individual pixels visible. So, $\mathrm{D}=2.6 \mathrm{~m}$ was chosen.

Moreover, by searching through online forums and consulting the software blending company it was found that up to $220^{\circ}$ of $\mathrm{HFOV}$ by using $3 \times$ Full-HD BenQ projectors is easily possible. Thus, $\mathrm{HFOV}=220^{\circ}$ was chosen, meaning there is an extra $40^{\circ}$ of FOV available to be added to the left side of the pilot in an asymmetric way. 
Solving Equation 2.11 for $\mathrm{R}$ gives the needed radius for the screen as:

$$
R=\frac{D \times \sin (\alpha)}{\sin \left(\frac{H F O V}{5.6}-\alpha\right)+\sin (\alpha)} \quad[\mathrm{m}]
$$

which was calculated to be $R=2.1 \mathrm{~m}$. Also $\mathrm{Y}=0.5 \mathrm{~m}$ and $\mathrm{D}=2.6 \mathrm{~m}$. The next stage was to implement the calculations into a CAD program to obtain a plot of the screen and determine whether the simulator cabin fits inside the circle having a radius of $2.1 \mathrm{~m}$, as shown in Figures 2.16 and 2.17. Since the distance between the projectors and the screen is constant and found to be $\mathrm{D}=2.6 \mathrm{~m}, \mathrm{Y}=0.5 \mathrm{~m}$ meaning that all the projectors should be placed in a circle with a radius of $0.5 \mathrm{~m}$ from the centre of the screen $^{1}$. Knowing the value of $\mathrm{Y}$ was critical to finding the exact locations of the projectors for the assembly stage.

Moreover, by drawing the thrown images of the projectors (by having the throwing angles) it became possible to evaluate the possibility of the cockpit shell blocking the images (i.e., causing shadows to appear on the screen). Using the CAD software, the height of the projectors has been chosen in a way such that there will be no shadow of the cockpit on the sides. As the projector is mounted higher, it must be tilted down more. On the front side, as the lower part of the image is hidden behind the hood of the cockpit and not visible from inside the cockpit, the existence of a small fraction of shadow is negligible. However, the reflection induced from the hood of the cockpit on the screen is disturbing which can be masked out by using the blending software, as shown in Figures 2.18 and 2.19.

\section{The Screen}

The process of designing and building the screen started with the choice of material. Initially, in order to keep the cost of the screen low, glass-reinforced plastic (GRP)

\footnotetext{
${ }^{1}$ It does not mean that the projectors should be placed $0.5 \mathrm{~m}$ from the screen (refer to Figure 2.17 )
} 
$6.1 \mathrm{~m}$

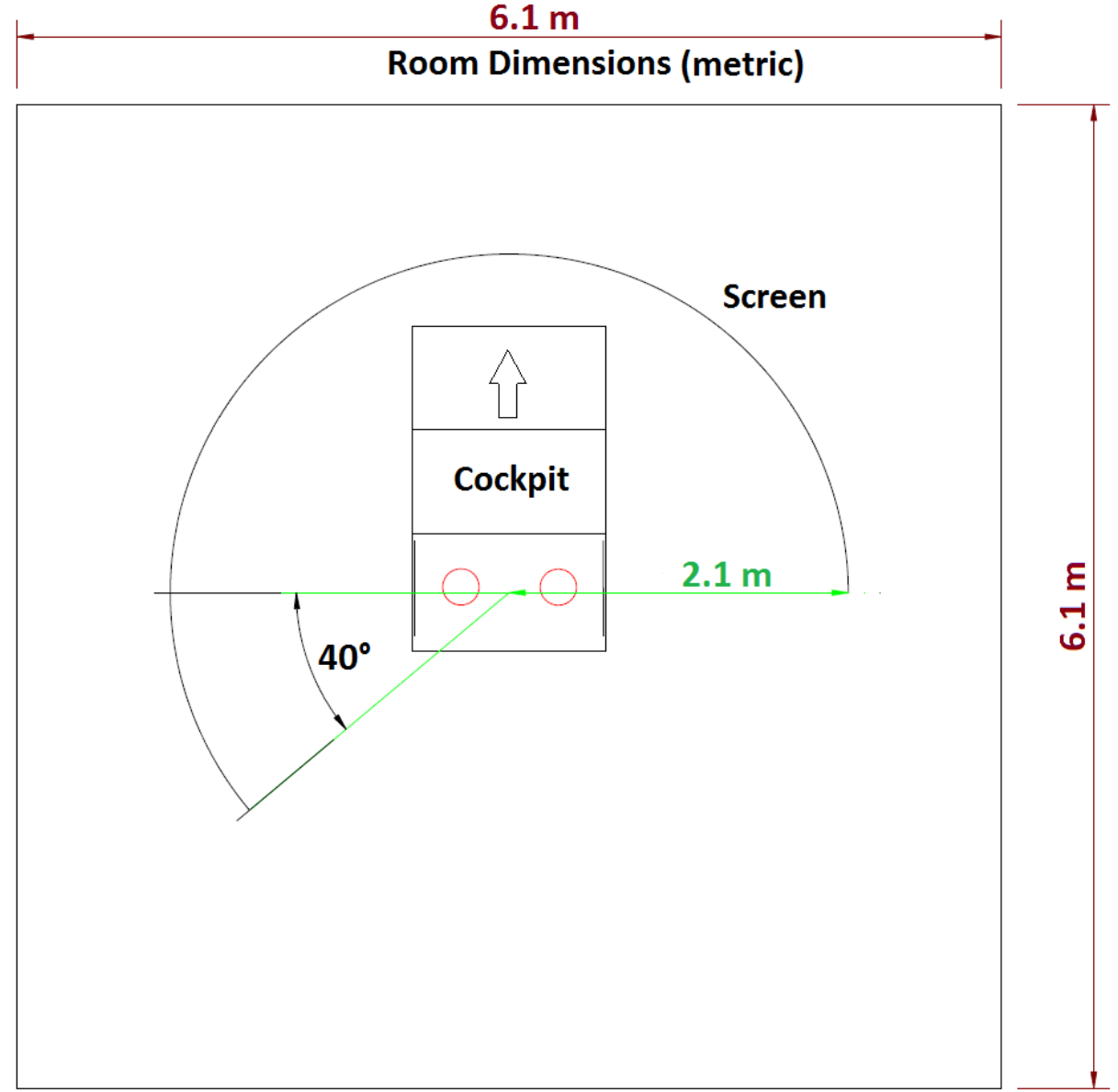

Figure 2.16: Simulator cabin fitting well inside the screen and the room dimensions. 


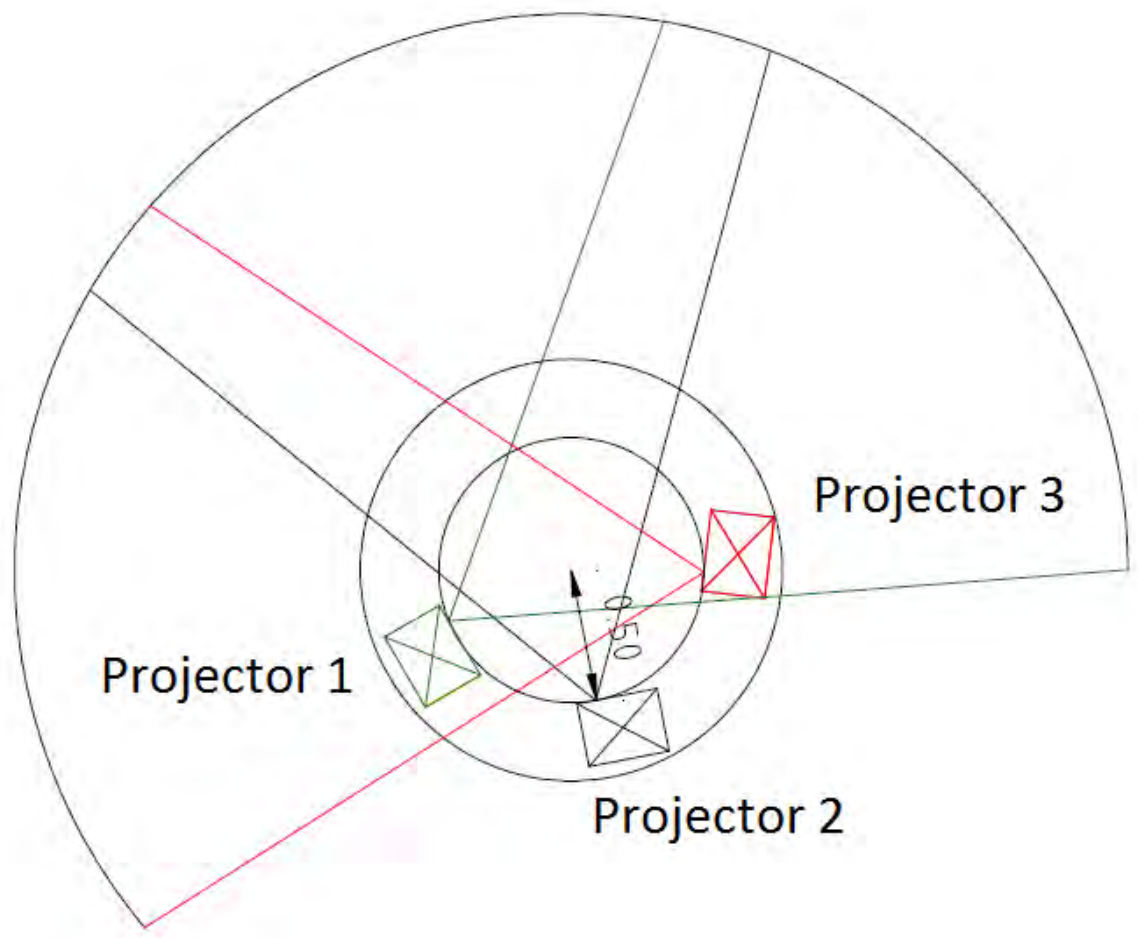

Figure 2.17: Top view of the projection setup with $10 \%$ overlap of the images.

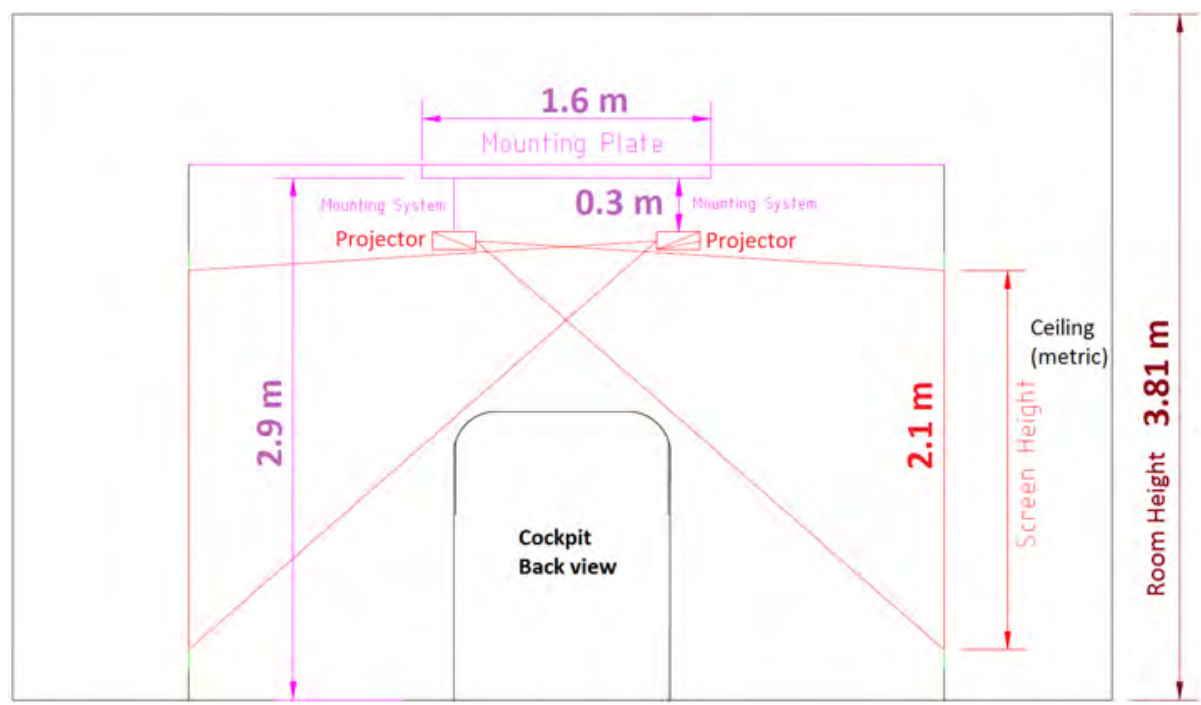

Figure 2.18: Shadow analysis for the CURVS visual system. 


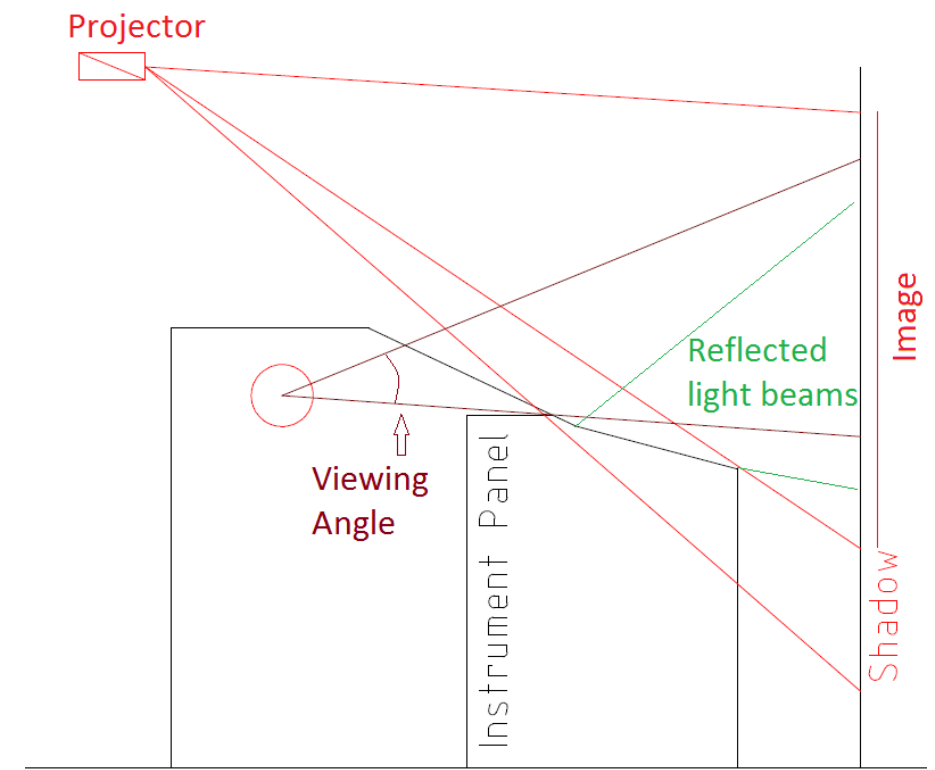

Figure 2.19: Shadow analysis in the front view. Note the reflections that must be eliminated.

material was found to be a good option. GRP is sold in rolls so that it could be ordered the size required and then bent to the desired curvature. Also, it could be painted white to produce a smooth white screen. The other similar option was medium-density fibre-board (MDF) that is thin enough so that it could be bent. MDF sheets are usually sold in fixed sizes and thus multiple pieces would have had to be attached to obtain a large screen.

The other alternative was to build a metal frame and attach a fabric over it. At first, this idea was speculated to be costly since the projector fabric with the required size and tube bending seemed to be expensive. However, a company named Carl's Place was found that sold multiple types of projector fabric for various applications [41]. After communicating with the company, they offered a reasonable price for the size and type of the fabric that was needed. The fabric they offered has the commercial name of FlexiWhite Projection Screen and is made of PVC materials. It is white in colour and has a gain number of 1.1 meaning that a brighter image in 
a dark environment where ambient light is controlled is achievable. As such, it was decided to purchase the fabric and build a frame to hold it and also design a mounting system for the projectors on the frame. It was also a better choice since the simulator was intended to be transported to Waterloo upon completion of the project.

Tubes can be separated and reassembled at the WWFC after the test aspects of the project were completed at Carleton University. Also, to hold the fabric tight it was important to induce enough tension on the fabric so there would be no wrinkles over its surface. The Carl's Place company offered specifically-designed bungees that would induce the intended tension on the fabric that has a finished edge with grommets, as shown in Figure 2.20. Detailed specifications of the fabric can be found in Appendix B.
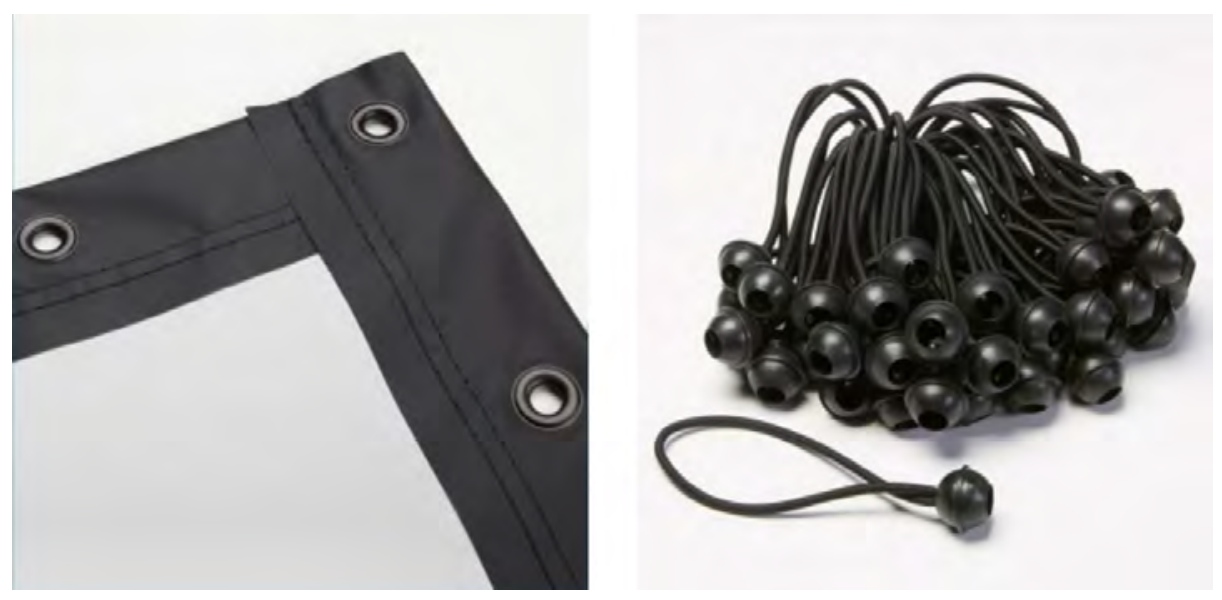

Figure 2.20: FlexiWhite fabric and the bungees for the screen.

The frame was designed and assembled with the help of another M.A.Sc student working in the ADL. Before performing a finite element analysis (FEA), as shown in Figure 2.21, to make sure that the structure will surpass a safety factor 2 against static load, it was important to select an appropriate material for the frame. Between steel and aluminum, steel was found to be a better choice because not only is it stronger for structures under tension, but also steel is significantly less expensive. All the tubes were ordered from a machine shop in Ottawa, named Ritchie's Welding Inc, 
where they had tube bending equipment by which some of the tubes were bent to the desired radius $(2.1 \mathrm{~m})$.

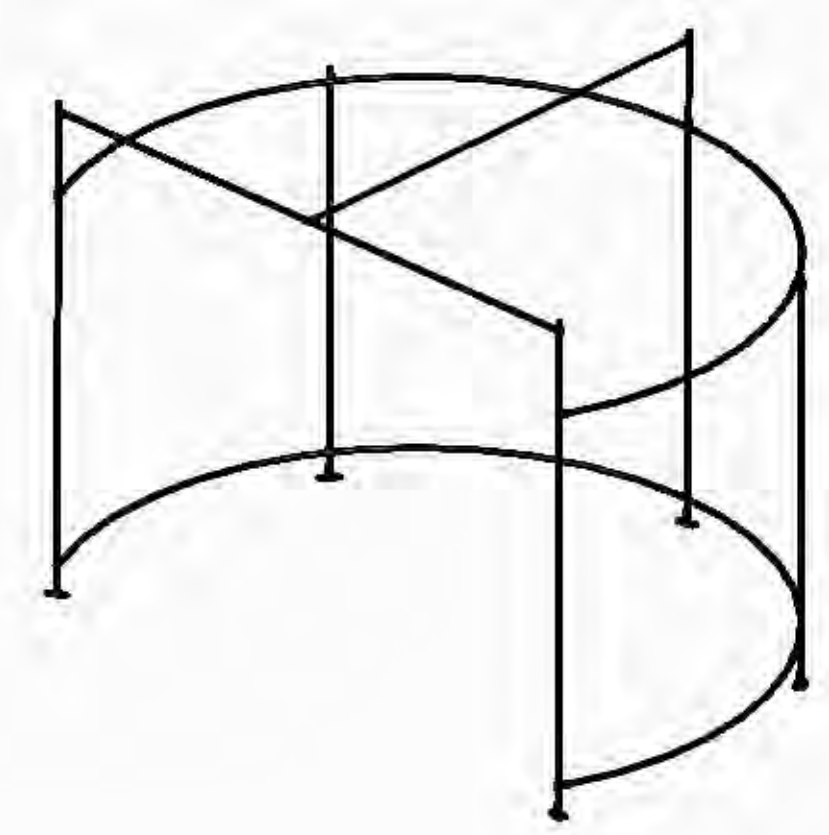

Figure 2.21: FEA analysis of the frame. Note the extra top bar was employed to support the projector mount.

In addition, the frame was built in pieces to ease the transportation from the ADL to WWFC by disassembling and reassembling it after transportation. There were a total of 7 straight tubes and 4 pieces of bent tubes that had to be attached together.

Also, the tubes arrived unpainted so they were painted in the ADL before assembling using black matte spray paint.

Tubes were attached using aluminum joints from a manufacturer named Kee Klamp [42], shown in Figure 2.22. Kee Klamps come in different shapes for various types of connections. Also, to connect the bent tubes, two custom aluminum components (machined at Carleton university) were added into the tubes where they meet, and then were drilled and bolted. This made the connections of the curved tubes quite accurate as illustrated in Figure 2.23. 


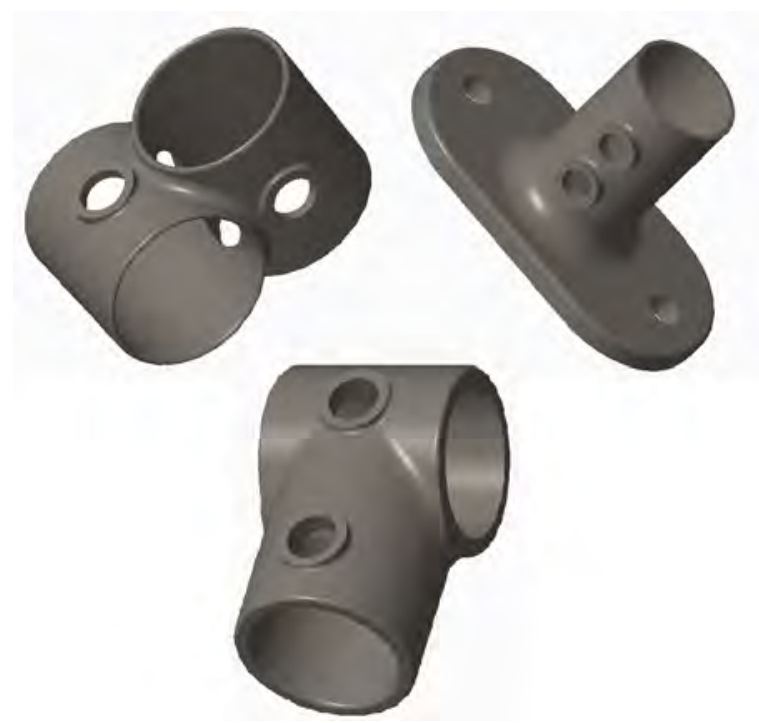

Figure 2.22: 3D model of the connectors (Kee Klamps).

For mounting the projector's, a plywood interface plate, shown in Figure 2.24, was chosen for two reasons: 1) light weight, 2) cost effectiveness. The possibility of mounting the projectors on a separate frame was also investigated. However, it turned out that designing a single structure takes less space and also is more cost-effective. The final assembly of the screen is shown in Figure 2.25. 

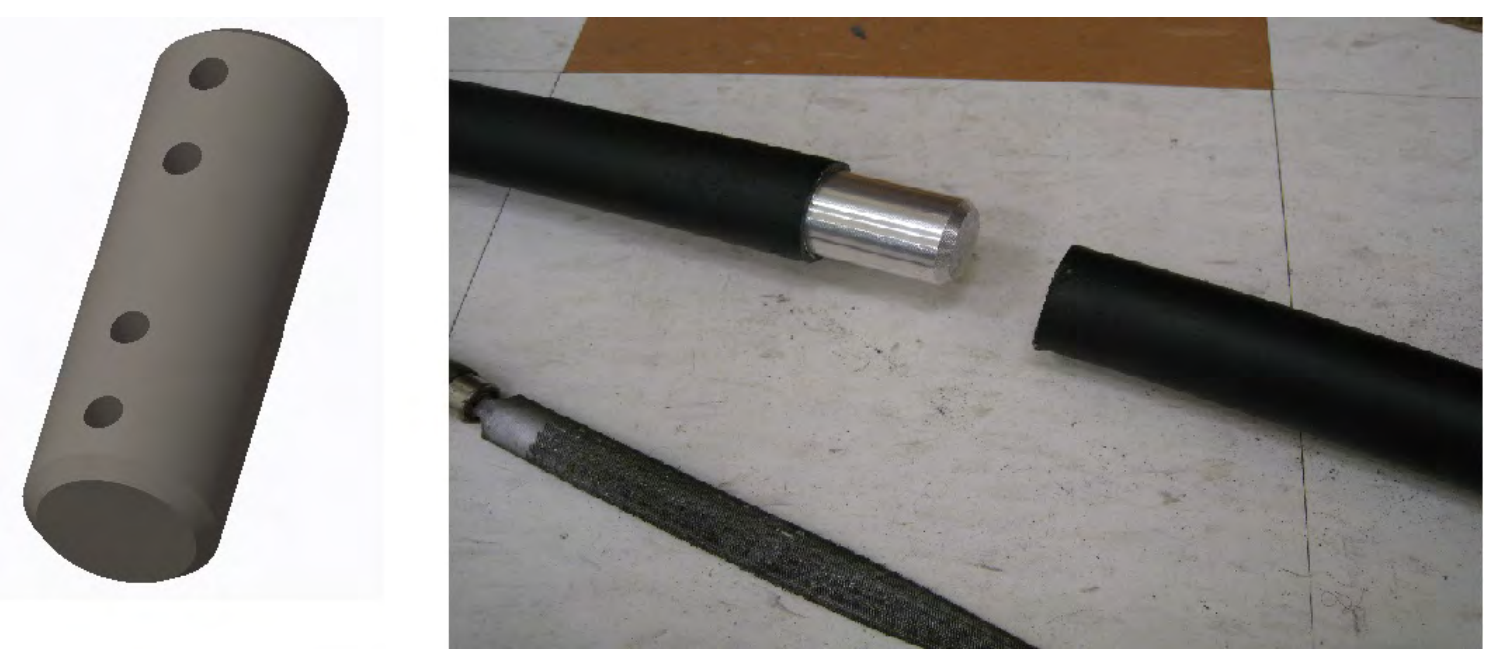

Figure 2.23: Aluminum component hammered and screwed to hold the curved tubes accurately (3D model on the left).

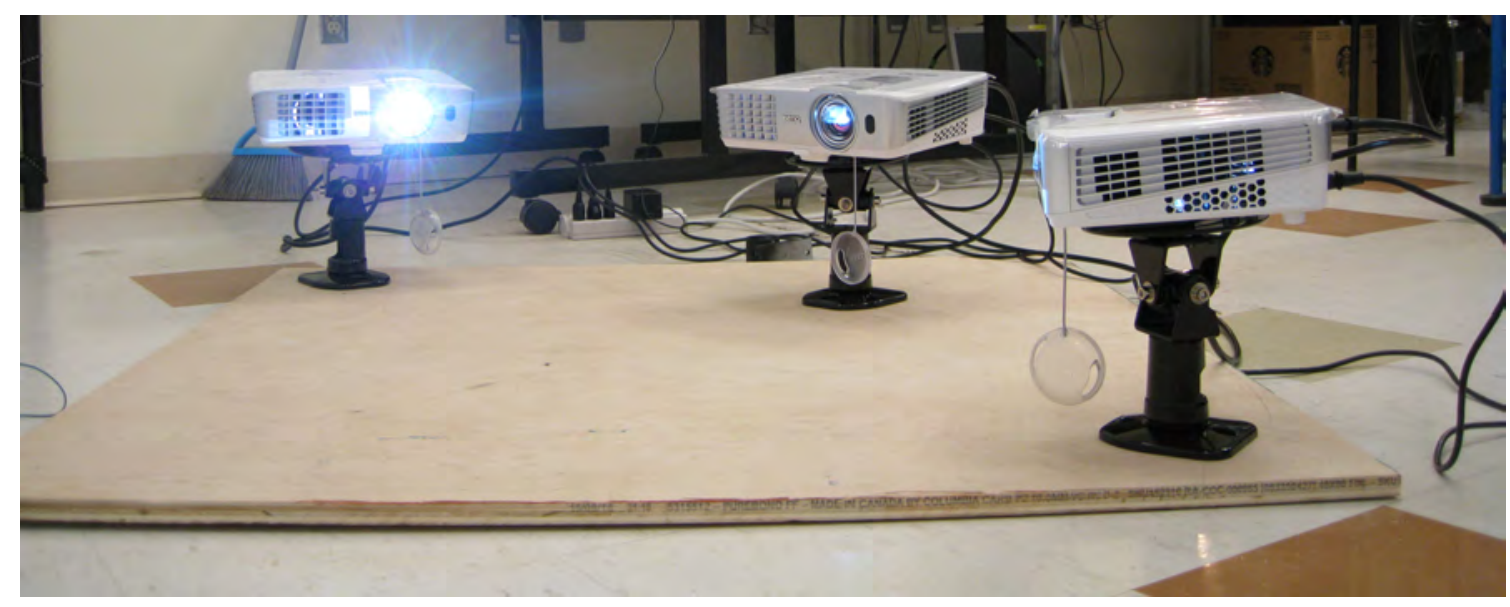

Figure 2.24: Plywood used for mounting the projectors.

The next challenge was the viewing angle inside the cockpit. As the original VectorSim had a very narrow FOV, only in front, its cockpit cover had a small window allowing only the front view. Therefore, it seemed necessary to cut the sides of the shell out like a real C172. For this purpose all the dimensions inside the actual C172 cockpit were measured, as indicated in Figure 2.27. The idea was to cut the windows so that the pilot feels the same as when viewing the interior of a C172. However, as OTW scene is shown on a curved screen that has limited FOV, there are some viewing 


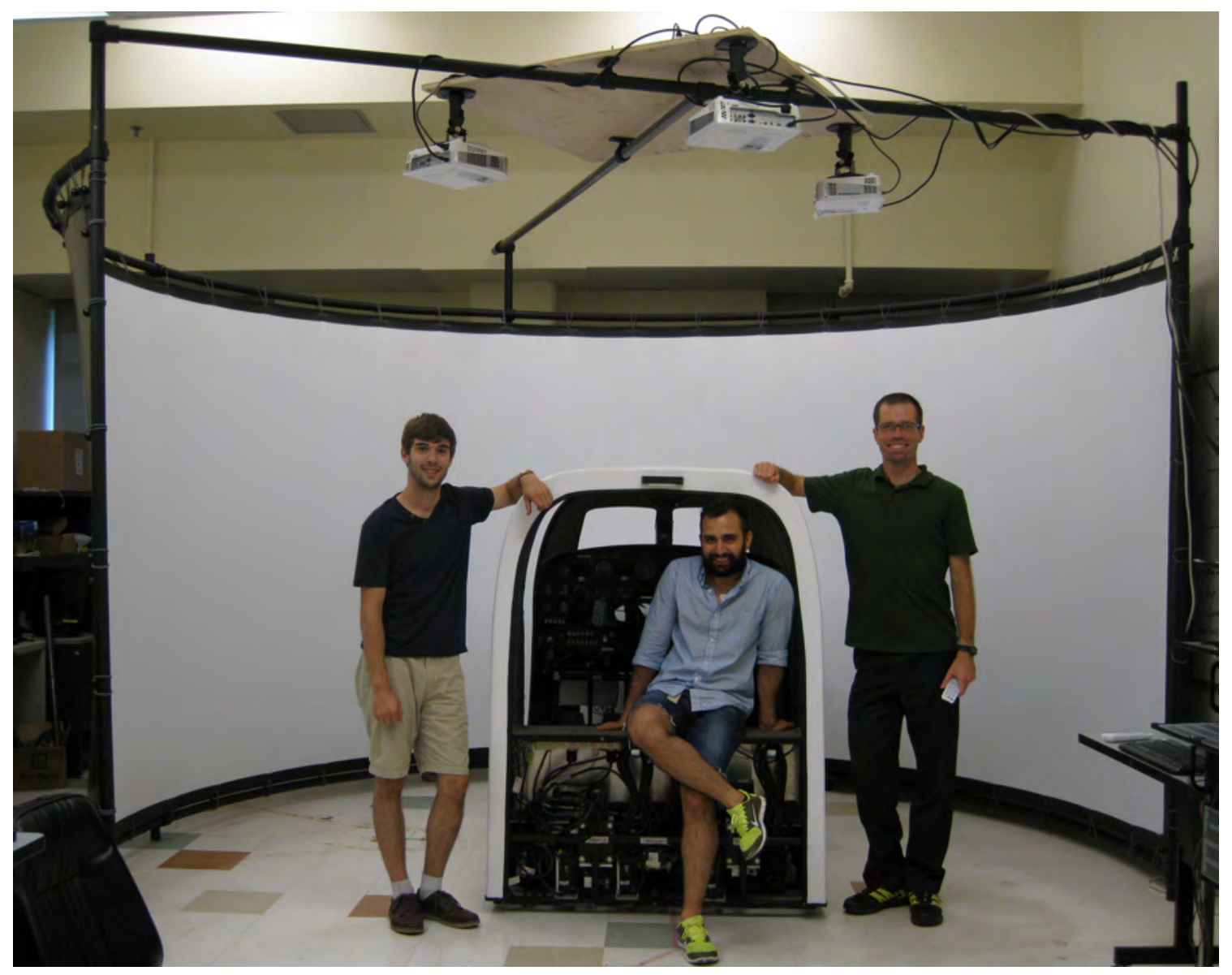

Figure 2.25: Final assembly of the CURVS screen with all the team members. 
angles through which the pilot might see the edges of the screen or the room's floor. To mitigate this problem, it was decided to build a replica of the cockpit shell using cardboard, shown in Figure 2.26 and cut the windows in a way that the pilot cannot see the floor, ceiling, or the edges of the screen. Nevertheless, the shell itself was not cut until the final preferred geometry was established.

Having the screen designed and built, it was time to incorporate it into the CURVS VCS and tuning it to the desired configuration that is discussed in the next section.

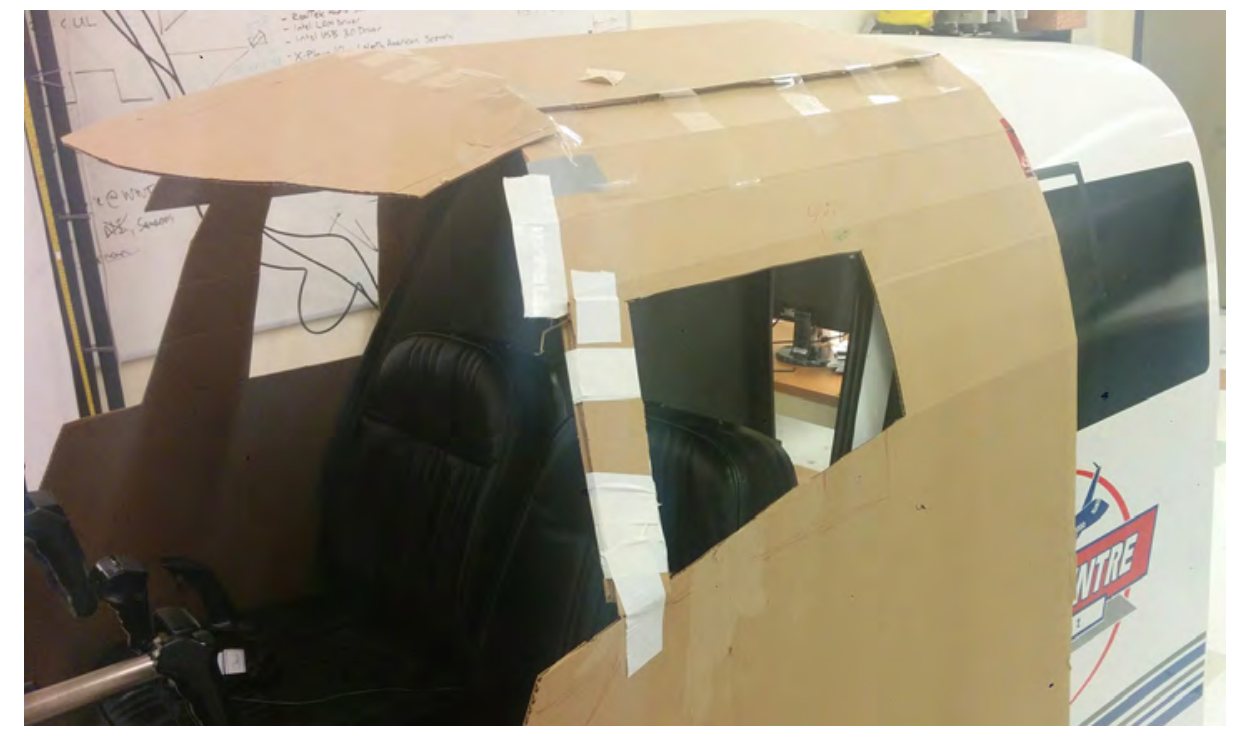

Figure 2.26: Cardboard mockup of the CURVS simulator.

\subsection{Customized Add-on Scenery}

One of the major and time consuming procedures for the CURVS VCS was the design of a customized scenery for the Wateloo aerodrome and its surrounding area and populate it with $3 \mathrm{D}$ objects. The idea was to replicate the same $3 \mathrm{D}$ environment as in the real-life, while not loosing many framerates. As the object density increases, more data is being rendered and as such there is a chance of reducing the framerates. 


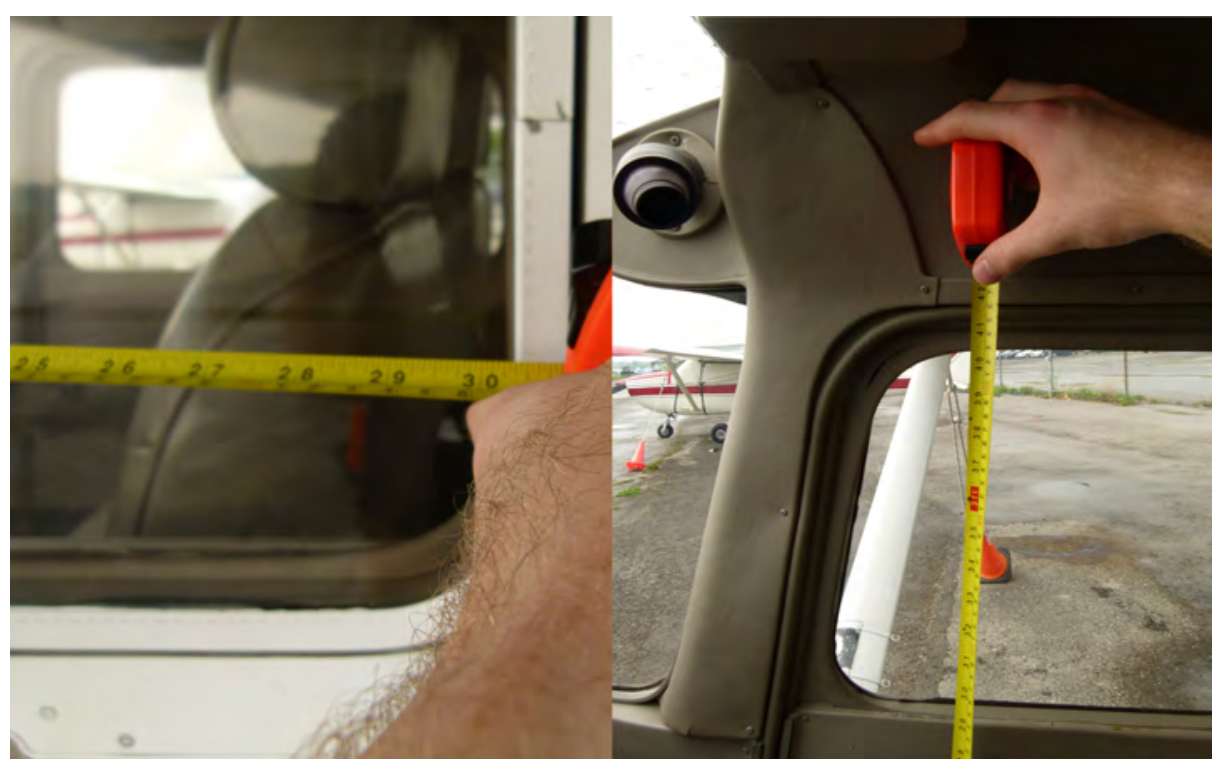

Figure 2.27: C172 aircraft window measurement.

$\mathrm{X}$-plane is delivered with a nonspecific scenery package that includes the world terrain data with generic textures, buildings, roads, rivers, lakes, seas, cities, airports, clouds, lights, sky, etc. Basically, its scenery content is made of tiles that define a cut of a specific latitude and longitude of the earth. On top of each tile there are terrain data, roads, objects, etc. that are recalled from a predefined object library. Additionally, external packages of scenery can be draped on top. The details about how X-Plane's scenery works is beyond the scope of this thesis. However, it is important to note that $\mathrm{X}$-Plane is released with external software and tools that enable the development of customized scenery packages.

Since CURVS is designed to be used at WWFC as a certified simulator, the scenery content for the Waterloo airport and the routes that are used in their flight training curriculum is essential. Therefore, one part of the project was devoted to design customized scenery for the Waterloo area. During the first stages of the project, WWFC indicated that flight plans from Waterloo to multiple destinations are used by students for practice. Therefore, it is useful to implement the scenery of all the destinations in their curriculum. However, designing all the airports needed for their 
routes is a stand-alone and time-consuming project. Hence, the focus was on finding the fastest and most efficient method that leads to realistic-looking scenery. Also, the scenery of the Waterloo airport has been designed to not only prove the design method, but also to provide high-detailed scenery content for practicing some basic manoeuvres such as circuits.

All the procedures to design the custom scenery surrounding the WWFC are summarized below.

\subsubsection{Aerial Data Collection}

The scenery was designed using photos of the area, satellite images, as well as a video that had been previously provided for commercial advertisements in the airport. Also, all the navigational aids (NAVAIDs), were available from the Canada Flight Supplement (CFS), a book that is the main resource for Canadian pilots [43]. These data include the runway headings, lengths, widths, longitudes, latitudes, marking and lighting systems as well as surface materials, taxiways, aprons, buildings, the location of hangars, wind socks, etc.

Moreover, multiple images of the area which had been taken by Brent Cameron, a CURVS project M.A.Sc collaborator, during a trip to the Waterloo Airport, as well as online resources were implemented to create a custom object library. One important online source was an X-Plane forum where experienced designers answer the questions of beginners to ease the design process [44].

To obtain the satellite photos or orthophotos, many resources have been tested. The most widely-used resource that X-Plane scenery designers use and also is recommended by X-Plane officially is the United States Geological Service (USGS) [45]. Nevertheless, the USGS database does not contain ortho-photos of Canada and it can only be used in the US. Every country has their own published geographical 
database that is accessible through the geographical information system (GIS) department in universities or federal departments. Canada and specifically Ontario also has an imagery database available for public use region by region. All areas of the southwest Ontario, that is the region of interest, is covered by a project called Southwestern Ontario Orthophotography Project (SWOOP) that gets updated every few years [46].

The latest SWOOP database that was available during the design process of the scenery was collected in 2010. Although the Waterloo airport has been further developed after 2010 and the need to use updated orthophotos was inevitable, for educational and test purposes SWOOP 2010 was selected to design the Waterloo scenery. SWOOP 2010 has $50 \mathrm{~cm}$ resolution such that every square pixel represents $50 \mathrm{~cm} \mathrm{x}$ $50 \mathrm{~cm}$ of land.

As discussed previously, X-Plane contains the world terrain data in itself. Therefore, the decision was made to not manipulate the terrain data. On top of the terrain and satellite images, X-Plane is capable of generating multifarious objects such as road networks, seas, houses, skyscrapers, etc. X-Plane utilizes its own specific algorithms to recall different objects from its object libraries and drape them on top which is not precise like real life. There are algorithms, however, that can be utilized to convert online geographical database formats like Open Street Map (OSM) into X-Plane geo-location format [47]. In other words, geo-location data of various objects, such as roads, lakes, forests, houses, etc., on the ground can be exploited and converted to XPlane format. The converted data are not the scenery content for X-Plane, but they are used to recall generic or custom-designed 3D objects from X-Plane's file library and locate them at the exact location as in real life. This method has the benefit that most of the road networks and infrastructure of the cities become relatively like the reality. This is beneficial especially for night flights using the simulator, as the road lighting network (which is a good visual reference for night flying) will be illuminated 
like the reality.

After some research on X-Plane's forum it turned out that someone else had already implemented this algorithm for Canada and had released the database as a package, called HD Mesh Scenery, that is free to use for the public [48]. Thus, it was decided to download and test the package.

The accuracy of the package had been proven to be viable by flying around the city and checking if the roads and objects matched with the satellite images. It was found to work accurately as shown in Figure 2.28.

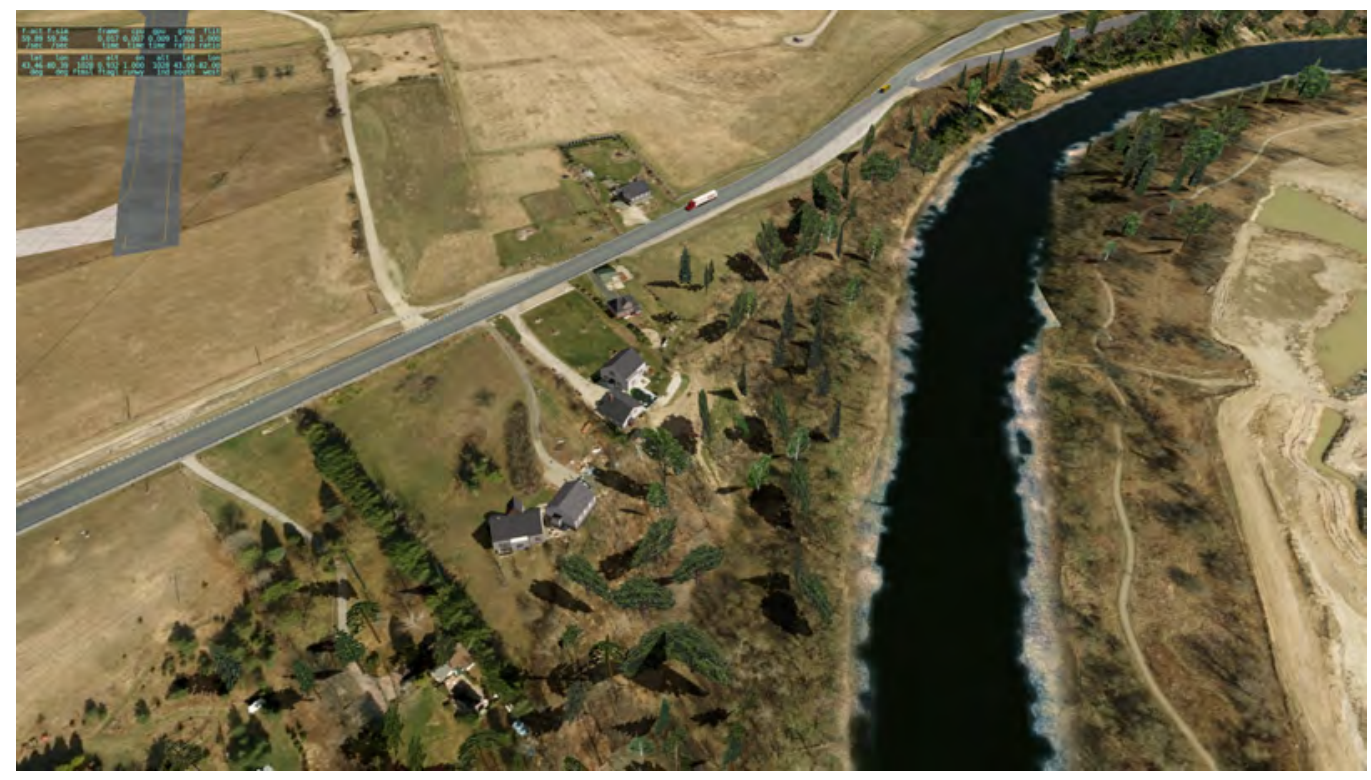

Figure 2.28: Open Street Map database converted into X-Plane and objects like roads, rivers, and trees generated the exact position on top of the satellite images.

The process of scenery generation in X-Plane starts with preparing tiles, then tiles are textured using generic textures of forests, land, cities, etc. or satellite images if any are available for that area. On top of that, generic 3D objects can be drawn. Also, custom-designed objects can be draped on top of all the layers.

In the end, X-Plane utilizes the scenery output in conjunction with its flight model, that is based on Blade Element Theory (BET), to generate a flow of images at $60 \mathrm{~Hz}$. 
The flow chart in Figure 2.29 illustrates this process thoroughly.

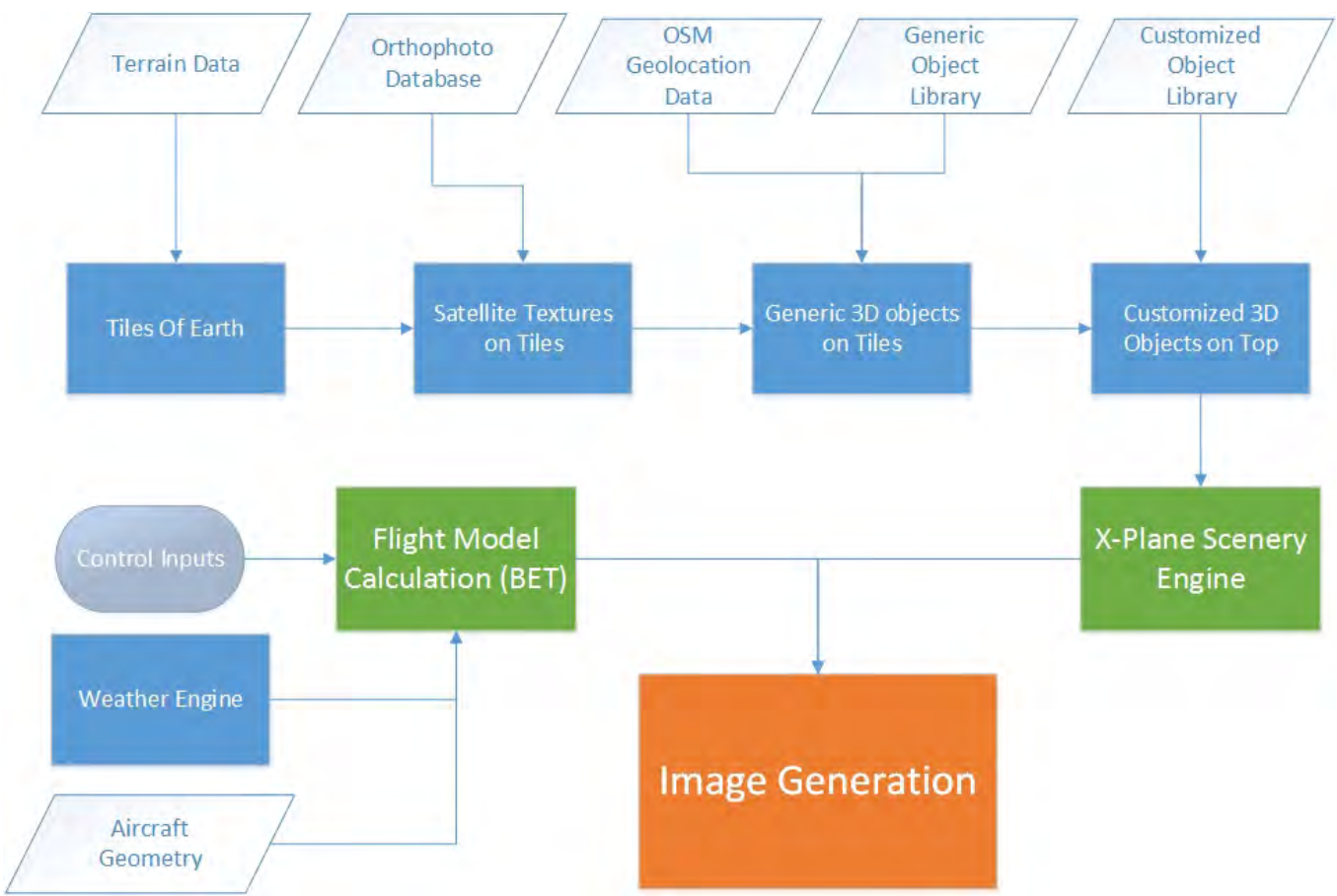

Figure 2.29: X-Plane image generation flow chart. 


\subsubsection{World Editor}

X-Plane comes with a scenery design and editing ETU tool called world editor (WED). Basically, WED is used to recall the 3D objects and textures from libraries and locate them on the ground and save them as custom scenery packages. Also, it is possible to upload the created scenery package for public use or to sell it. The first step to use WED is to draw the satellite imagery on the ground and that requires having suitable satellite images in GeoTIFF format. GeoTIFF is a public domain metadata standard which allows georeferencing information (i.e., the coordinate system) to be embedded within a TIFF file. WED can read GeoTIFF files and automatically maps the satellite imagery to the exact location as it is on earth. See Figure 2.30.

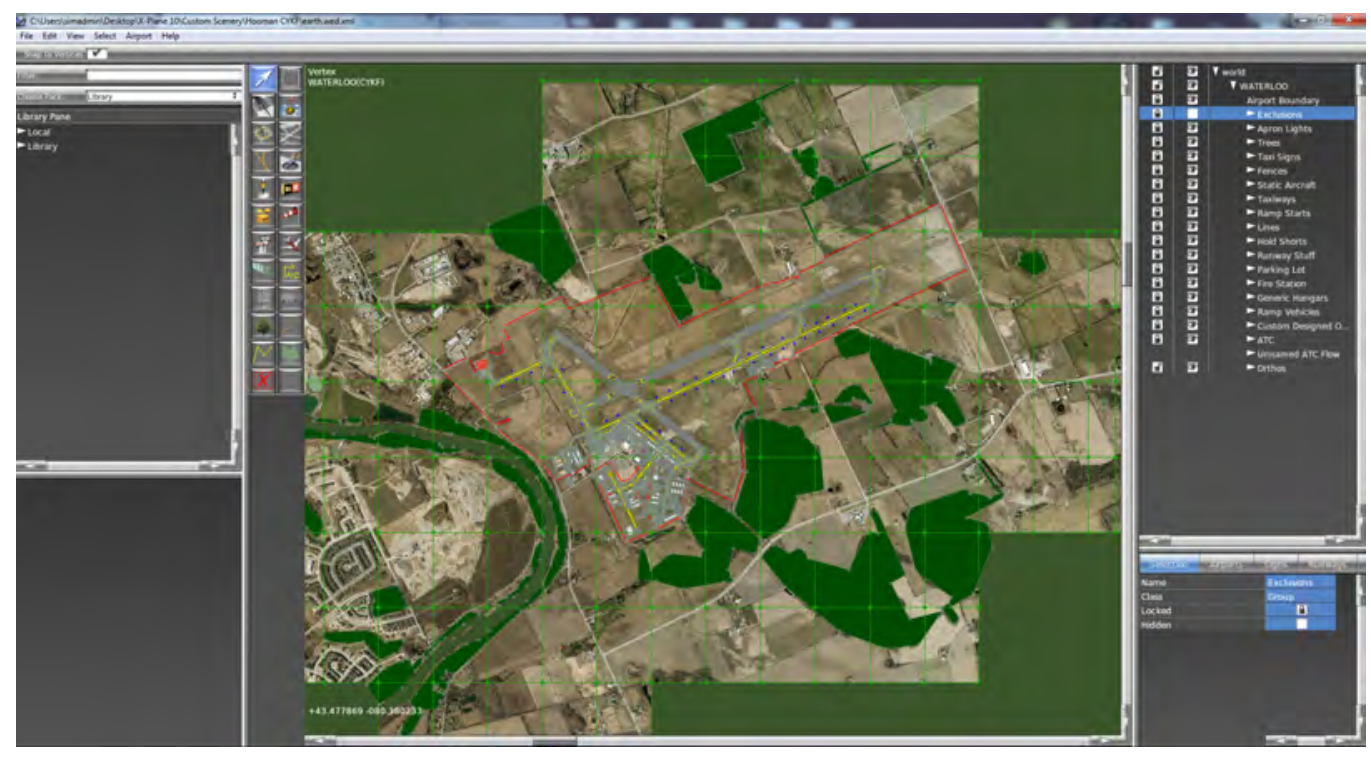

Figure 2.30: WED interface.

Next the runways, taxiways, taxi-lines etc. can be drawn on top where physical characteristics of each of those have to be determined in WED. Using the same method, 3D objects can be positioned in the right spots.

There exist multiple software packages by which custom 3D objects such as hangars, terminals, parking lots, etc. can be designed. 


\subsubsection{SketchUp}

In flight simulation design, very detailed buildings or facades are not recommended for two reasons. First, as aircraft fly all the scenery content will be viewed from large distances and thus their details will rarely be observed. Second, the more detailed the object file, the more computation power it needs to be rendered which might cause latencies in real-time simulation.

Moreover, as designing an airport with all its facilities and buildings is a time consuming process, there was a need for 3D modelling software that is ETU and fast. SketchUp was utilized as the 3D modelling computer program. SketchUP is a free, fast, and ETU program that has a wide variety of applications by which both high-detail and low-detail models can be created [49]. Also, SketchUp comes with a plugin that easily converts the $3 \mathrm{D}$ file format to the one that X-Plane makes use of ${ }^{2}$. Therefore, all the custom 3D objects were designed using SketchUP. See Figure 2.31 as an example.

\subsubsection{Aircraft Component Modification}

Before an aircraft takes off, the pilot always checks the ailerons as a part of the before-takeoff checklist to make sure that controls work properly. Also, as the C172 is a high-wing aircraft some of the VFOV on each side is blocked by the wings. Also, in the front view, the propeller is visible in a C172 which helps the pilot determine if the engine is running without the need to look at the engine gauges. Therefore, it is very practical to have a complete dynamic wing view (moving ailerons) on the sides as well as a dynamic rotating propeller in the front view.

\footnotetext{
${ }^{2}$ SketchUP can generate .OBJ files, and also X-Plane uses .OBJ files; however, they are different file formats with the same extension and therefore the SketchUP.OBJ version was needed to be converted to the X-Plane version.
} 


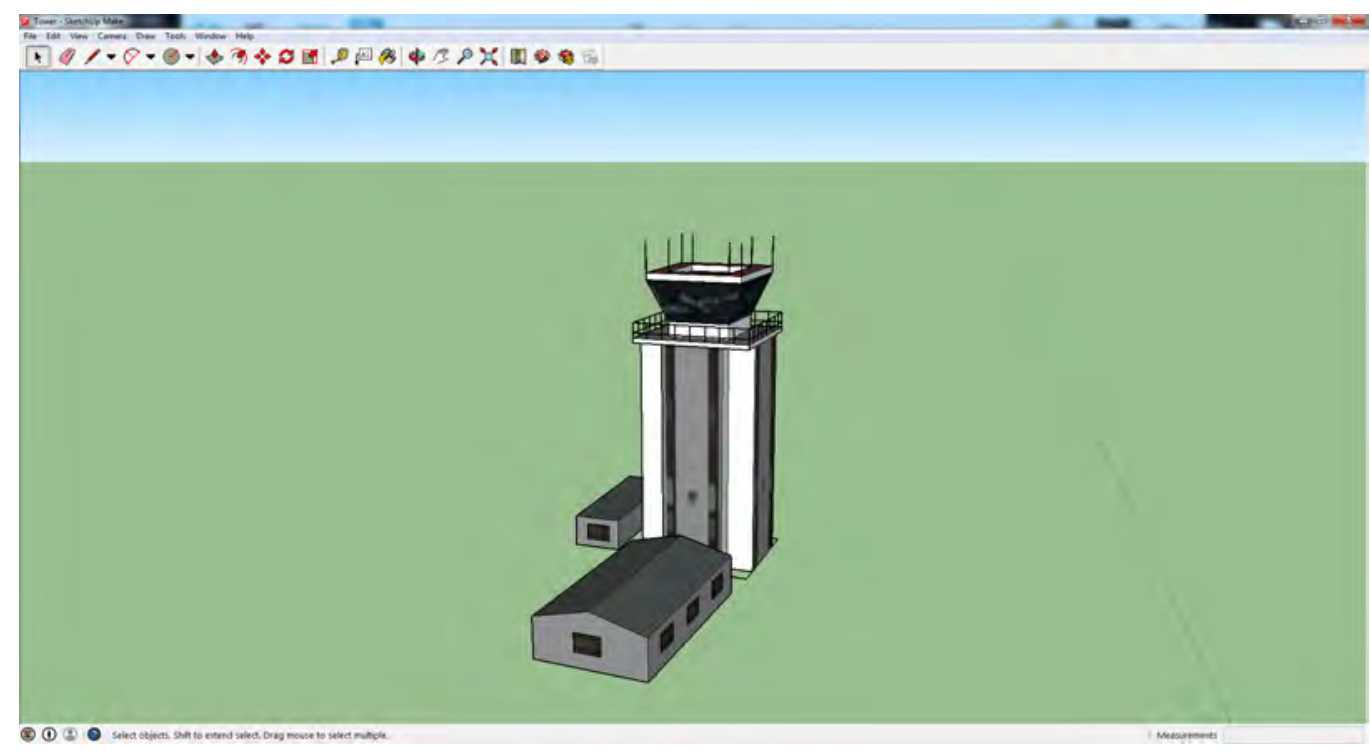

Figure 2.31: The design of the Waterloo International Airport control tower using SketchUp.

To do so for the CURVS, the C172 3D model that originally came with X-Plane had to be modified. The aircraft model in X-Plane consists of two components: visible interior and exterior models, and the geometric characteristics for flight model calculations. It means that the 3D model of the aircraft that is visible in the simulation environment is different from the one that is implemented for flight dynamics. Therefore by manipulating only the visible model of the aircraft it is possible to change the model as desired without affecting the dynamic behaviour of the aircraft. This was done using PlaneMaker, a tool for aircraft mathematical model design in X-Plane [39], and as well as SketchUp for editing.

SketchUp was used to remove the components that are not desired to be seen on the screen such as the 3D cockpit and interior components of the aircraft (as there is a physical cockpit in the simulator) and PlaneMaker helped to define the PEP in the aircraft. The result is shown in Figure 2.32. 


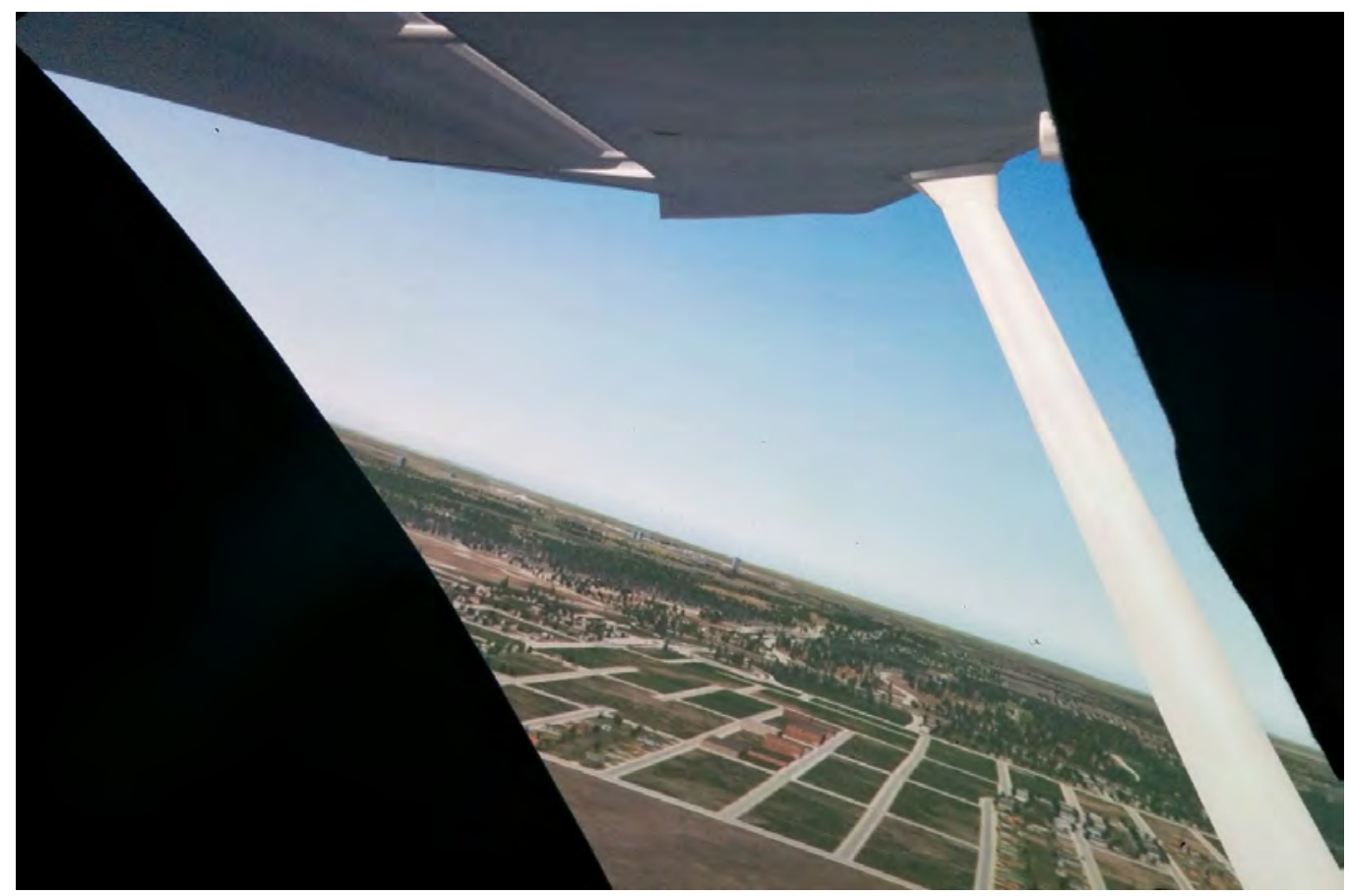

Figure 2.32: The dynamic wing view in the CURVS visual system.

\subsubsection{Resulting Scenery}

In this section, some screenshots of the scenery with details in their captions are shown in Figures 2.33 through 2.40. Also note the frame rate expressed in FPS that is almost 60 in every view; however, in the airport area there is usually a drop in FPS due to $3 \mathrm{D}$ object density. 


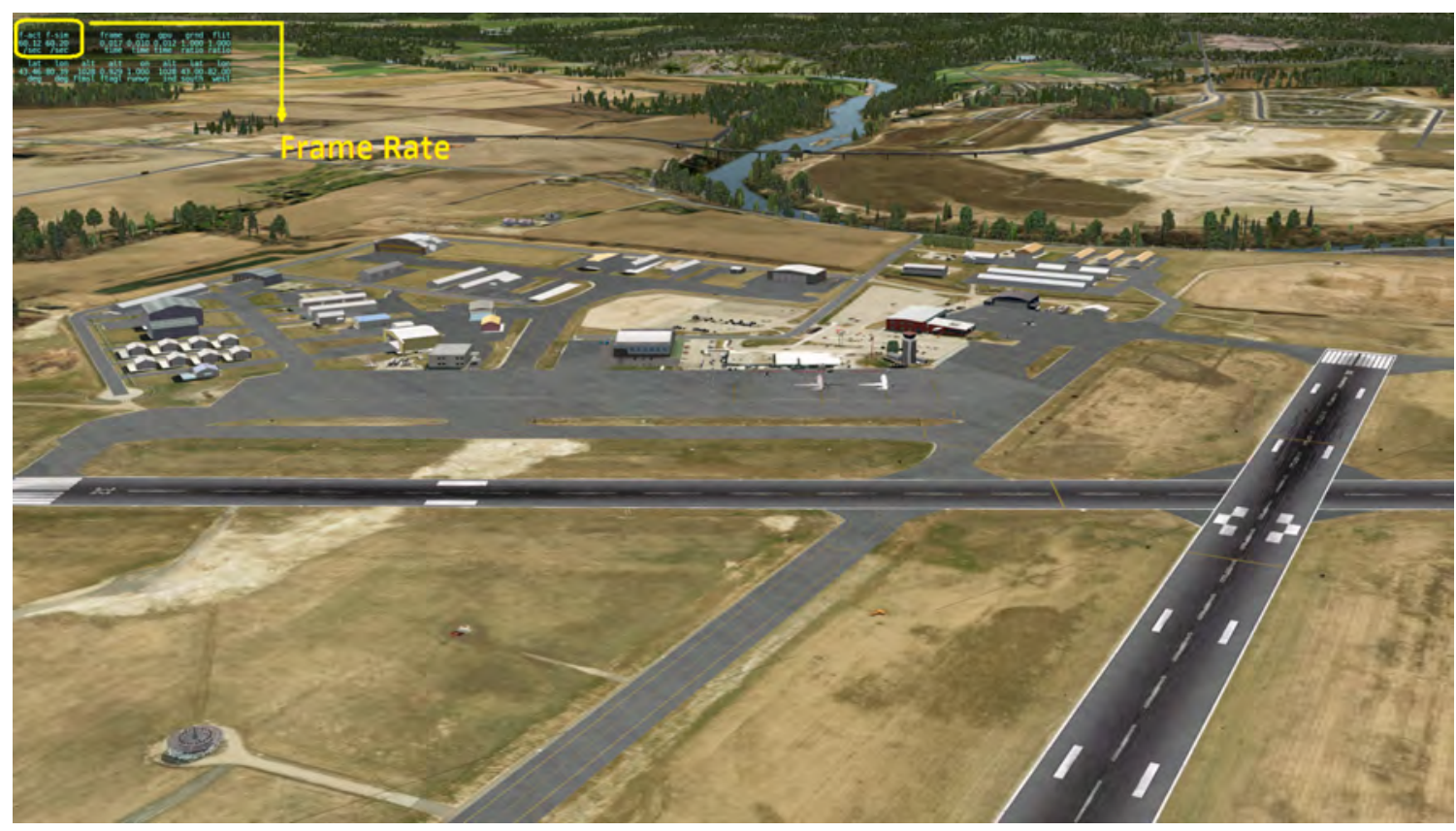

Figure 2.33: Waterloo airport (CYKF) custom-made scenery overview. Note the framerate is 60 FPS in an area with high object density.

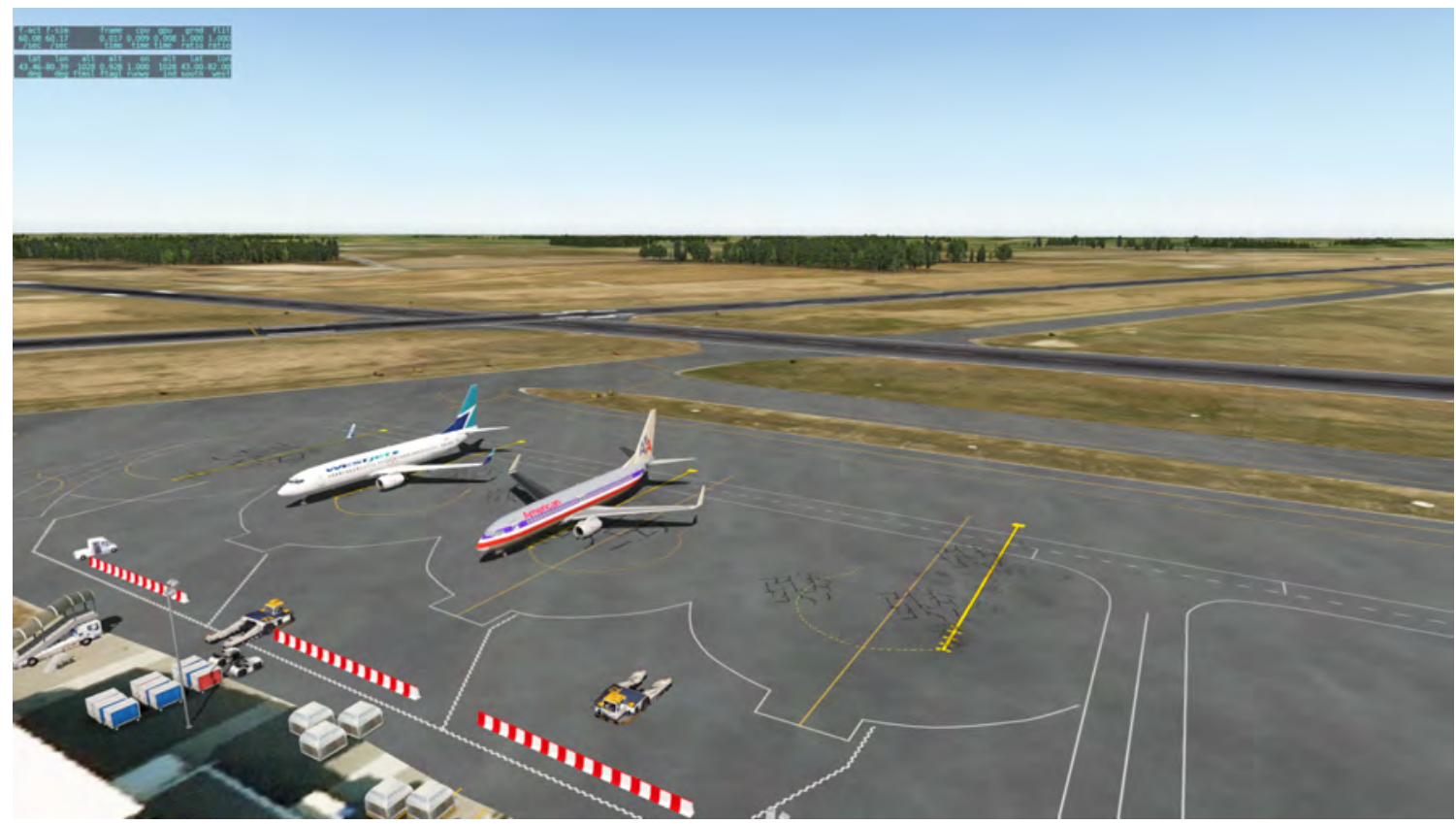

Figure 2.34: Waterloo airport (CYKF) main terminal apron custom scenery. 


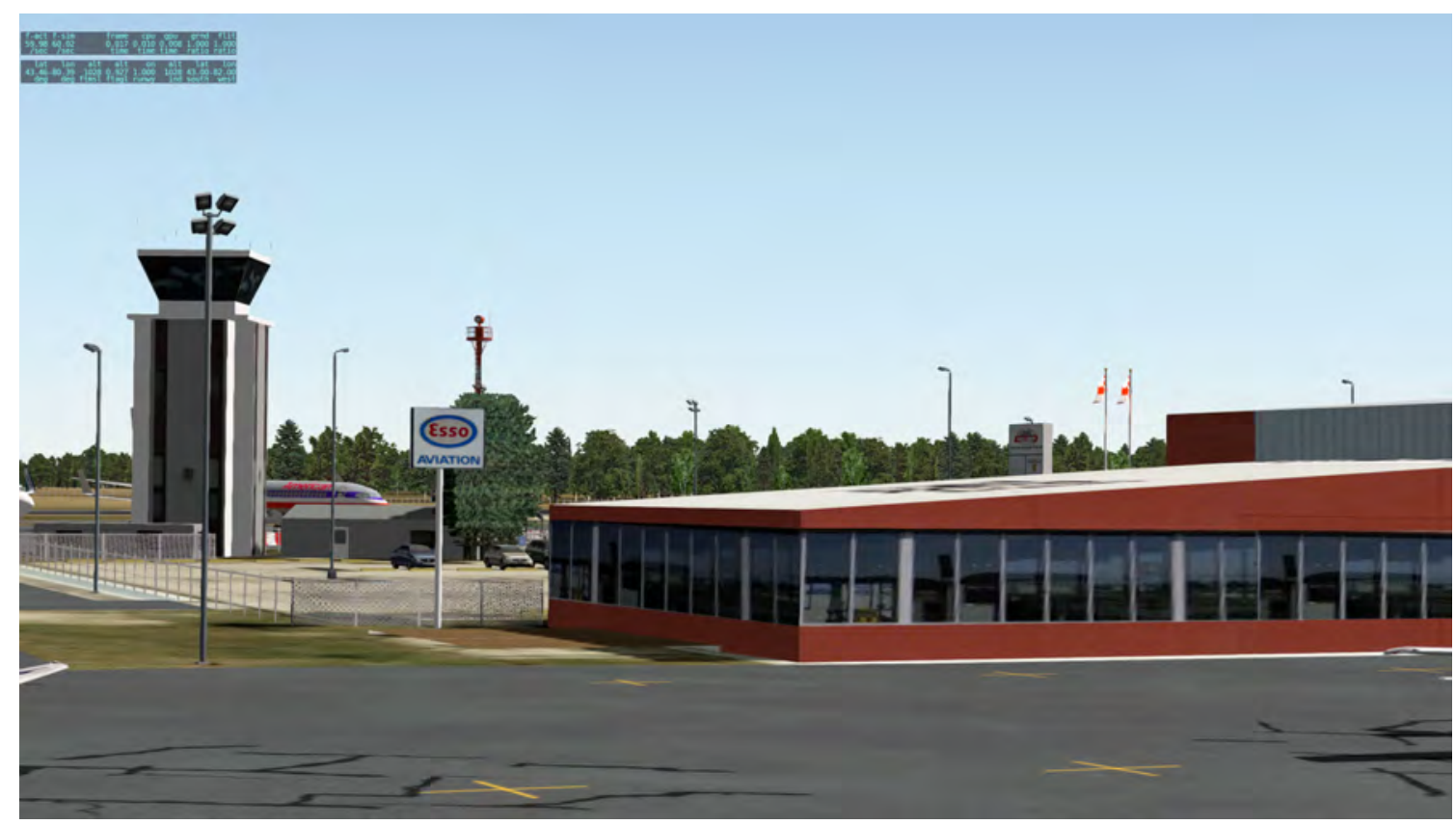

Figure 2.35: A view from the WWFC parking lot of the Waterloo Airport (CYKF) custom scenery looking toward the control tower.

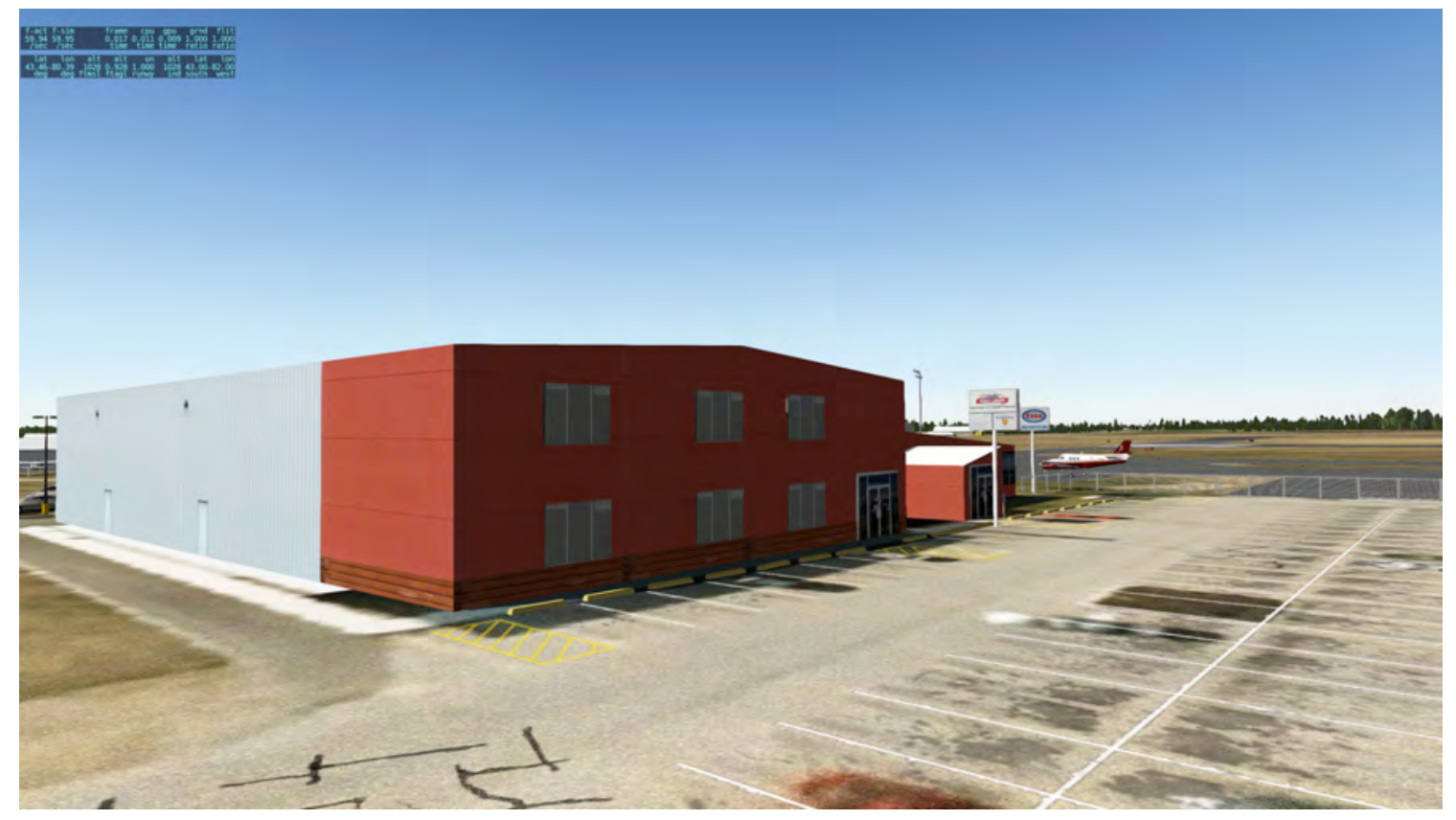

Figure 2.36: Waterloo Airport (CYKF) WWFC main office building custom scenery. 


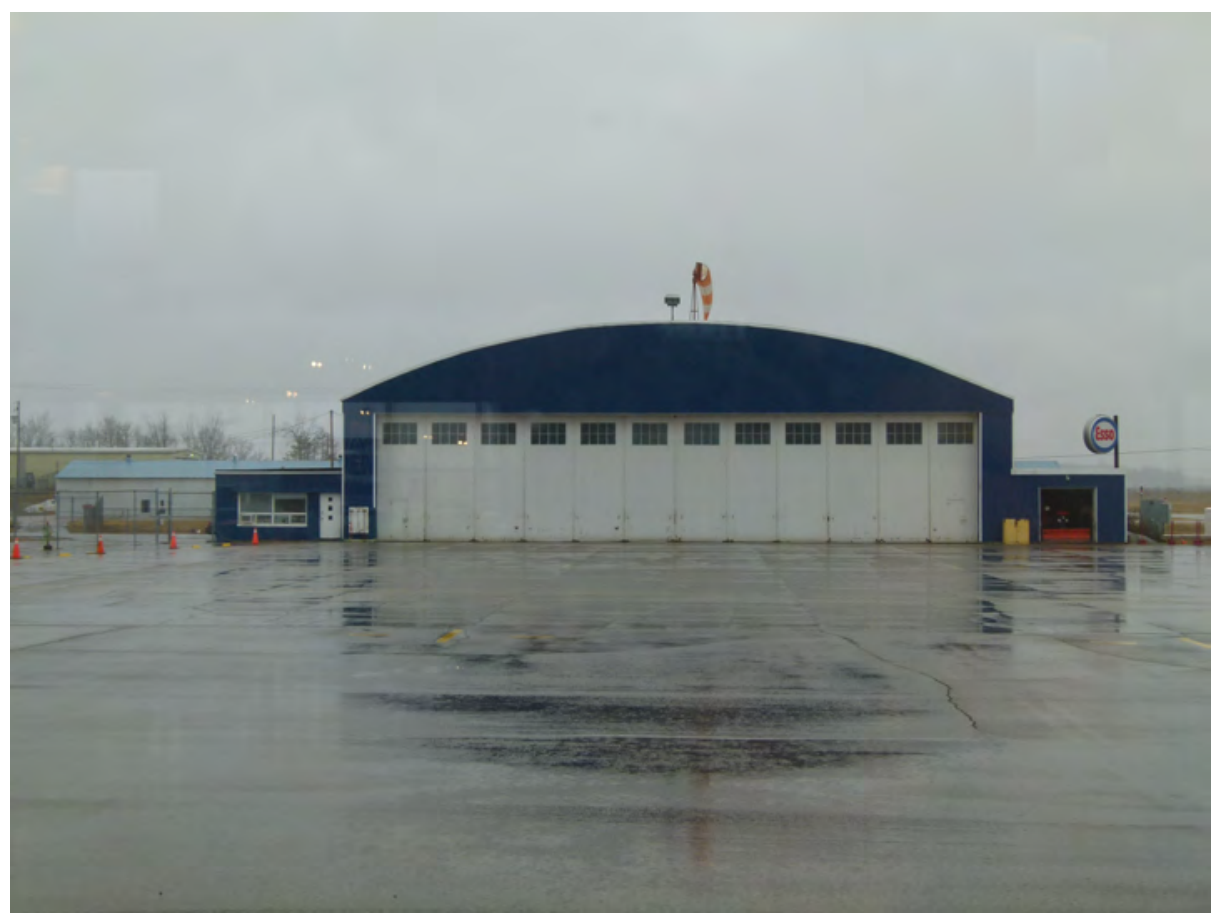

Figure 2.37: A picture of the WWFC parking lot (real).

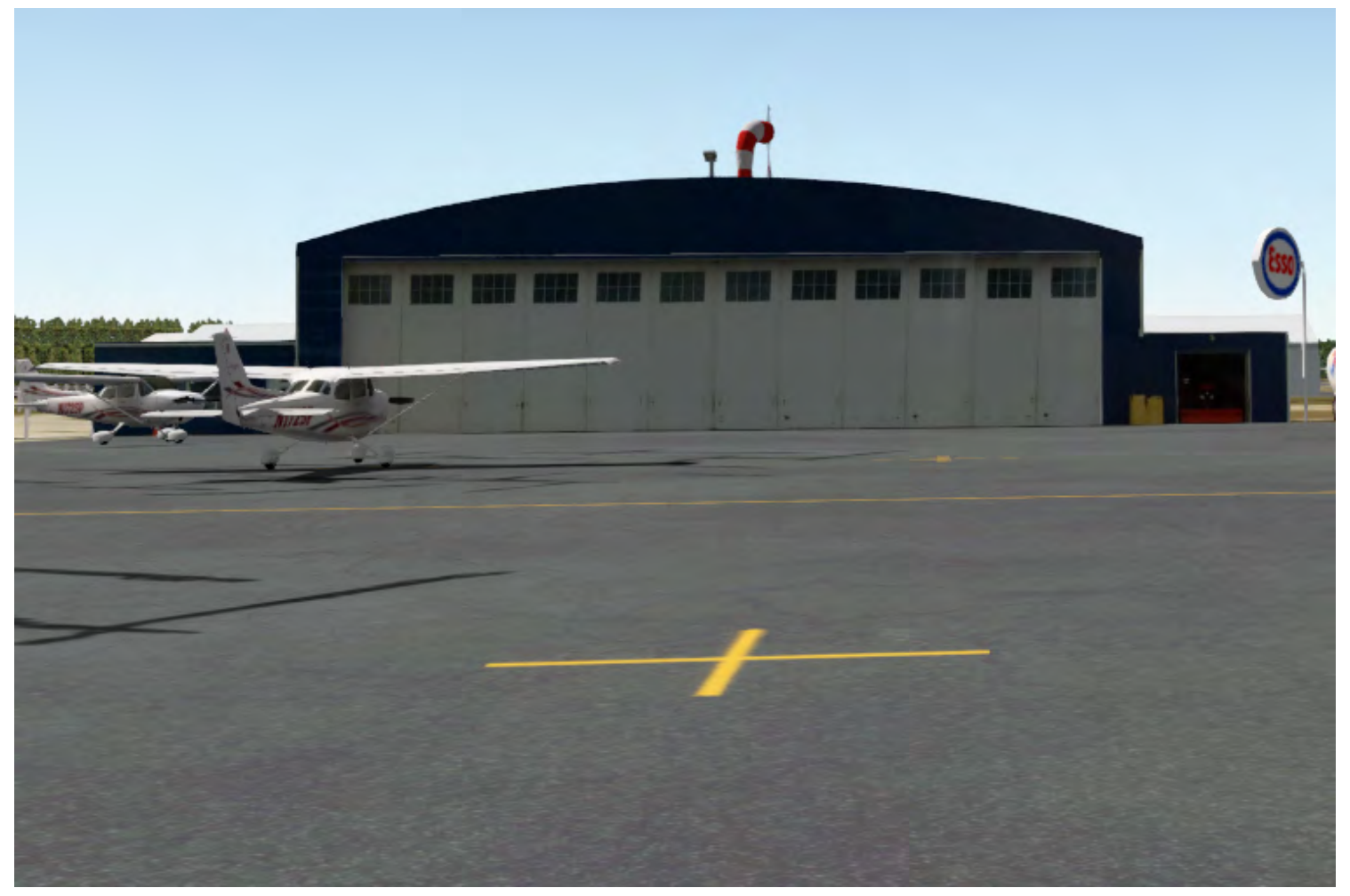

Figure 2.38: The same spot as Figure 2.37 in the simulated environment. 


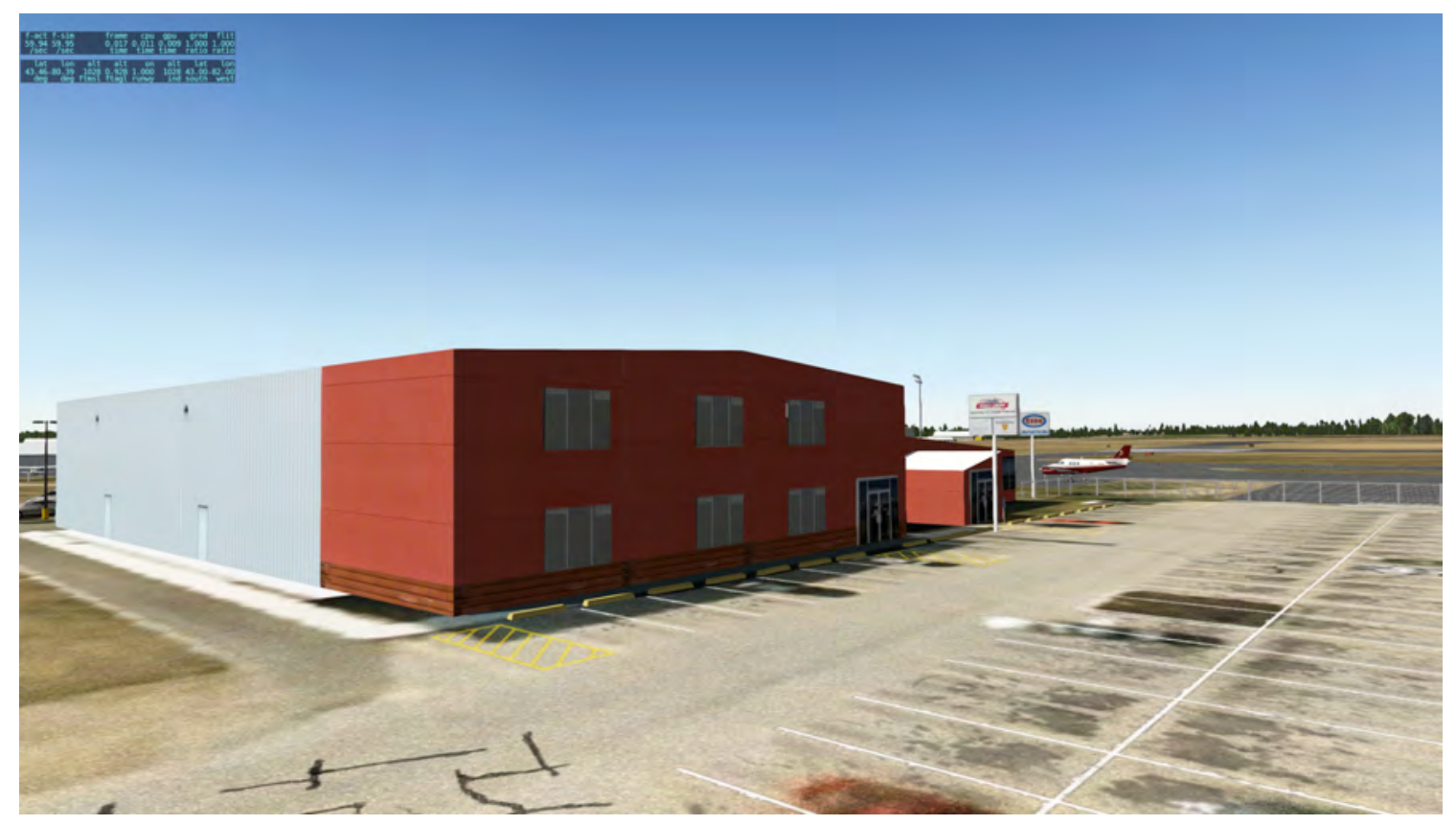

Figure 2.39: Waterloo airport (CYKF) WWFC main office building custom scenery.

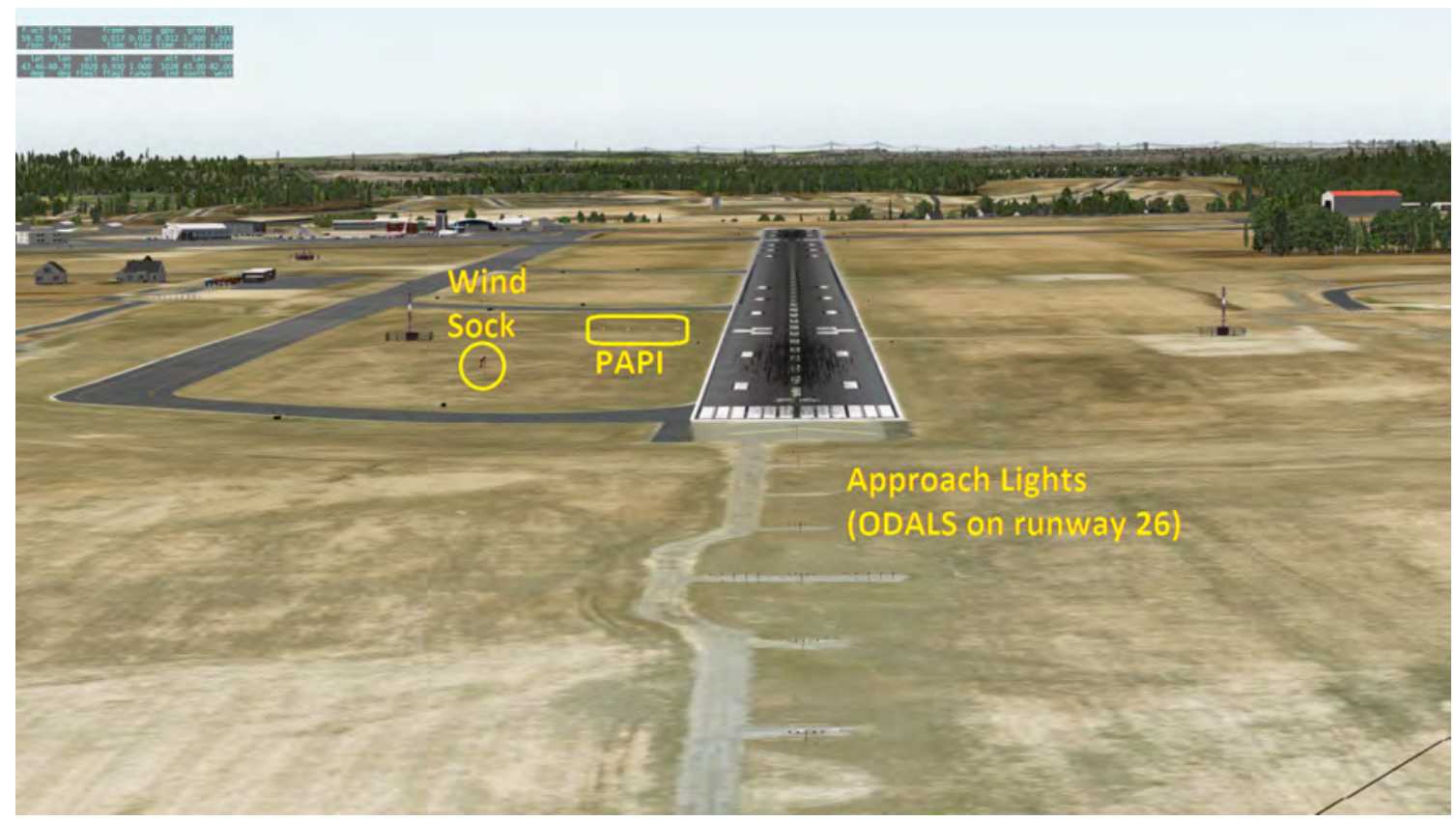

Figure 2.40: Waterloo airport (CYKF) custom Runway 26 with PAPI lights, ODALS approach lighting system, and animated wind sock. 


\subsection{Tuning and Evaluation}

\subsubsection{Geometrical Correction and Soft-edge Blending}

The blending of the projector's images was performed using COTS software named Immersive Display Pro (IDP) [50]. IDP can be implemented for various types of projection screens. It has the capability to correct the geometrical distortion of the images on cylindrical, dome, or double-curved screens and combines and blends the images together smoothly. One other advantage of IDP is that it has the capability of fading out the edges of the blending region so that the brightness of the projectors does not accumulate. This eliminates the need for using shutters in front of the projector lenses that create artificial shadows. The profile of the faded parts can be adjusted manually. Moreover, this software can combine the images of parallel networked PCs that are specifically designed to render X-Plane or MSFS.

For calibration, IDP comes with another software program, Immersive Calbiration Pro (ICP), that does the calculations and saves the result as an specific file format to be read by IDP on each computer. There are two types of calibration method embedded within the ICP: 1) Live-camera calibration; and 2) Manual calibration.

In both methods, it is important first to enter the shape and size of the screen accurately in the software. Second, the ICP projects an image that consists of rows and columns on the screen through each projector. Columns and rows are numbered so that each projector has a distinct set of numbers of rows and columns. In camera calibration mode, a live-camera (such as a webcam) that can view the screen collects the position of the columns and rows. Thus, the software can calculate how far offset the images are projected using sophisticated image processing algorithms.

In the manual mode, however, the rows and columns are projected on the screen for the operator to manually correct the distortion and blend the images by repositioning the columns and rows using the mouse and keyboard, as shown in Figure 2.41. 


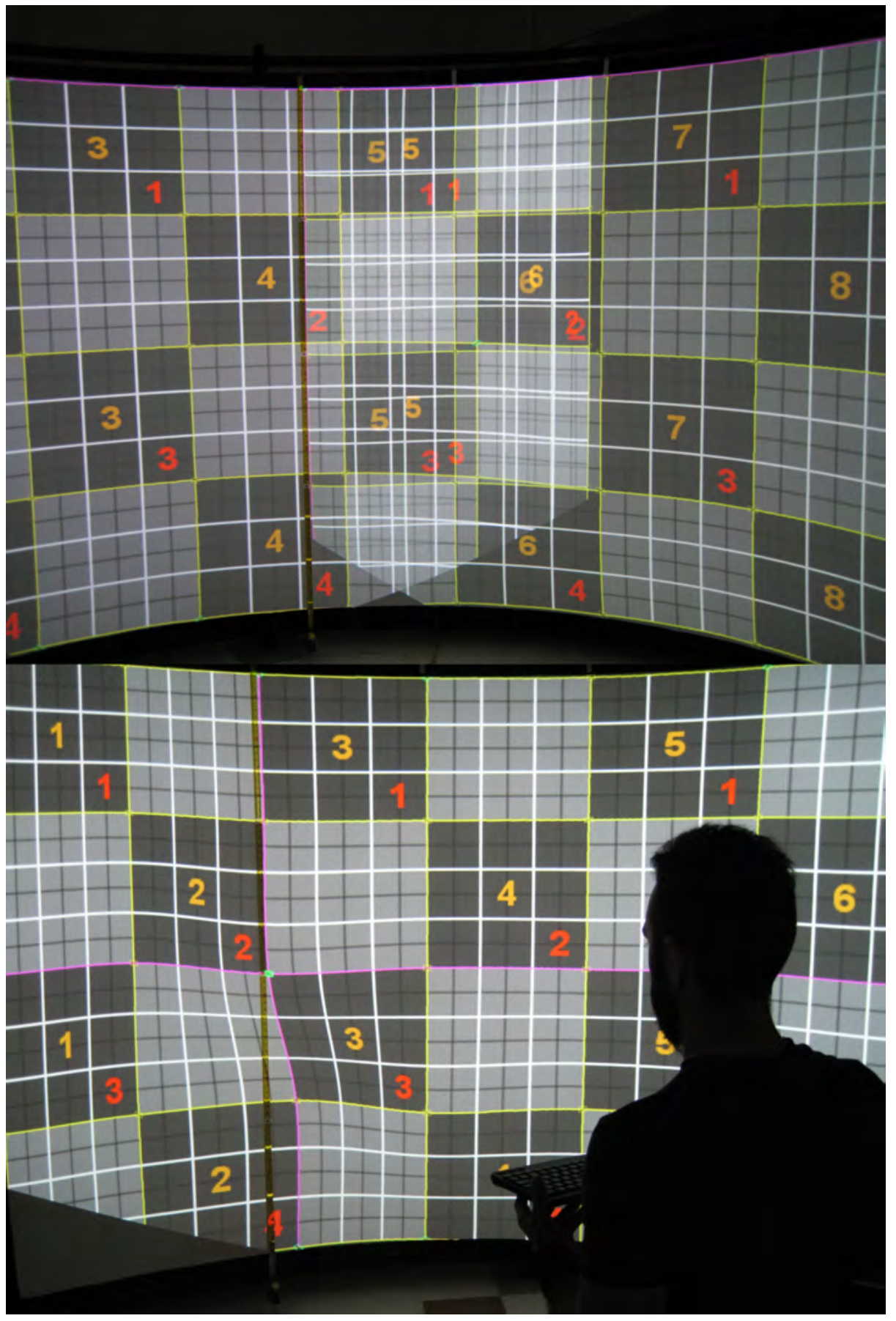

Figure 2.41: Manual calibration of the images using Immersive Calibration Pro. 
In order to calibrate the screen both methods were implemented to determine the most effective approach. Although the camera calibration was found to blend the images more accurately, the extensive curvature of the CURVS screen prevented the webcam from capturing the entire screen, thus only half of it could be calibrated. To address this, a fish-eye lens webcam was implemented. It turned out, however, the result was not satisfactory as fish-eye lenses are not linearly warped. As such, the very centre of the screen was accurately corrected but the edges of the screen became stretched. Therefore, it was decided to use the manual mode of the software.

In the manual mode, it is required to choose a number of rows and columns (of your choice), and the software calculates the width and height of each column and row, respectively, for the operator that is based on the size of the screen that has to be given to the ICP in advance. Then the operator has to mark the screen using those specific distances. Next, the software projects the columns and rows on the screen such that they can be repositioned using the mouse and keyboard to match the marked labels. The specific number of rows and columns and their distances for the CURVS screen can be found in Appendix C. Figure 2.42 demonstrates the final test pattern of the software and Figure 2.43 shows X-Plane running with the IDP software. After the calibration is performed and tested, the results can be saved in separate files for each computer. The files, in fact, include the geometrical correction and fading profile of each computer's projector. Each computer reads the file using the IDP software independently allowing it to stream visually-corrected imagery.

It is also important to note that the ICP can prescribe for X-Plane the PEP. Therefore, it also calculates the content for and writes a text file in which the HFOV, VFOV, and required offsets of the screen, should be defined for each computer separately. 


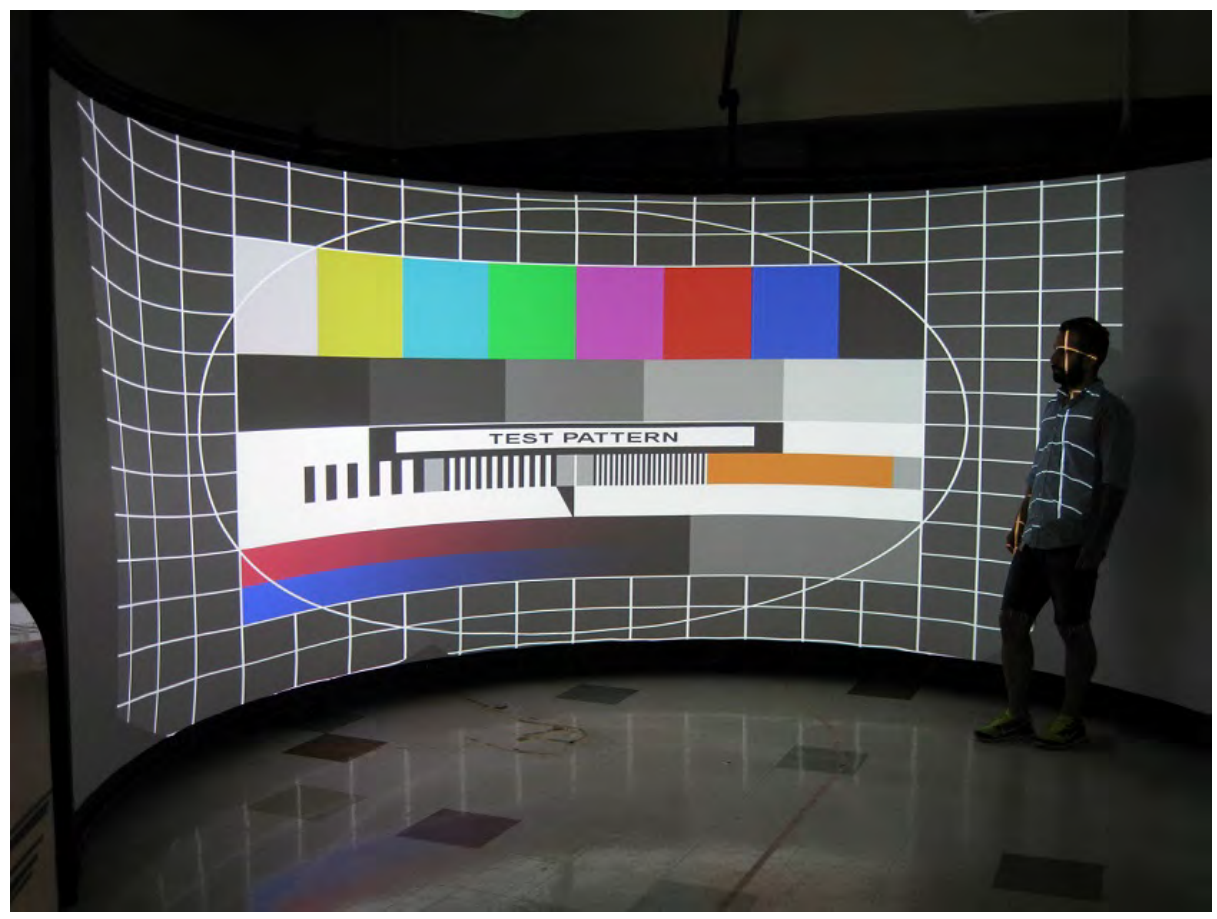

Figure 2.42: Calibrated screen test pattern.

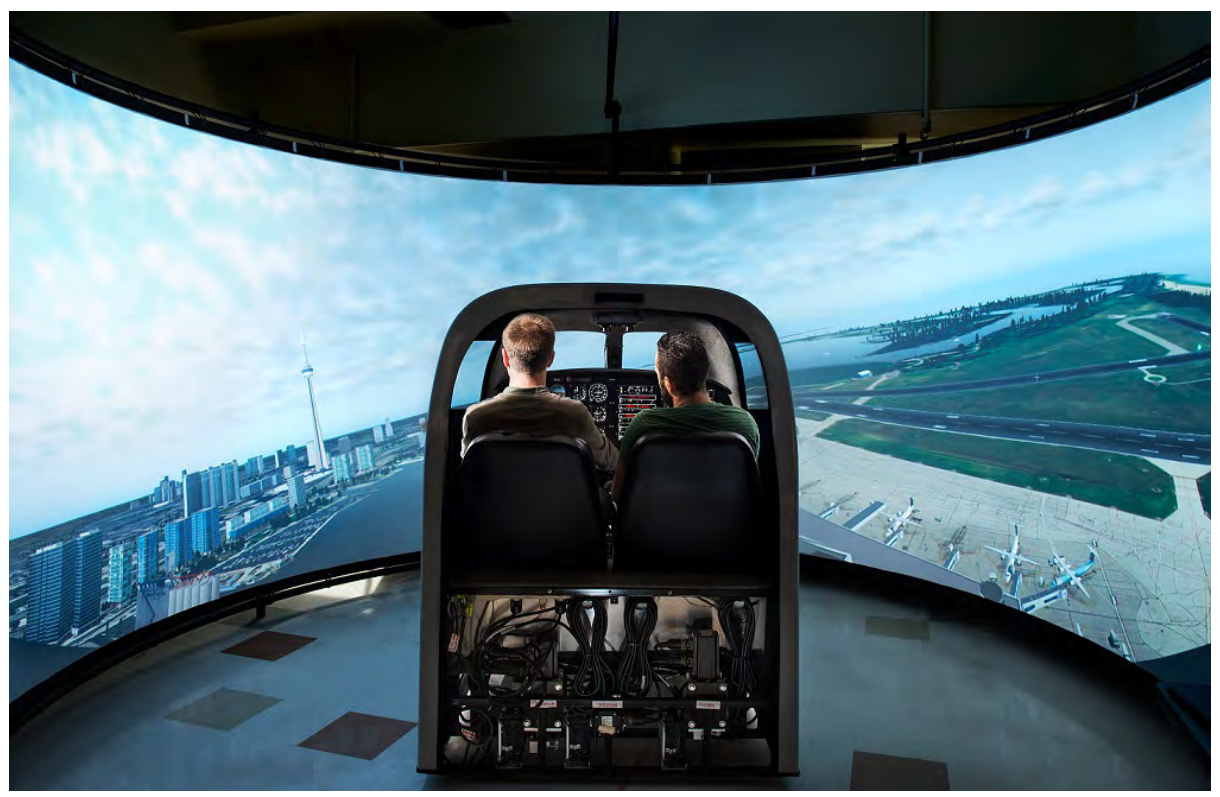

Figure 2.43: Final result of the calibrated screen with blended images.

\subsubsection{Screen Resolution}

The screen resolution was measured using a ruler, measuring the size of each individual pixel. In a perfect screen resolution scenario, every pixel should be around 1 arcmin 
(average human eye's acuity) so that the image does not look pixelated. The absolute screen resolution can be easily calculated by having the distance of the PEP from the screen and the size of each pixel. Using this method the resolution of the CURVS VCS has been calculated as 2.8 arcmins which exceeds the International Civil Aviation Organization (ICAO) manual for recommended simulator's resolution limit (less than 4 arc-minutes) [31].

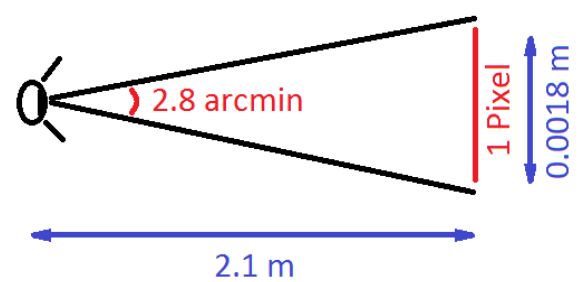

Figure 2.44: The calculated resolution of the CURVS VCS.

\subsubsection{Parallax Evaluation}

The parallax angle in the CURVS VCS is measured using CAD software and found to be 8.24 degrees which satisfies the ICAO criterion (less than 10 degrees with the capability of switching the eye point between the pilot and the co-pilot seats) [31]. 


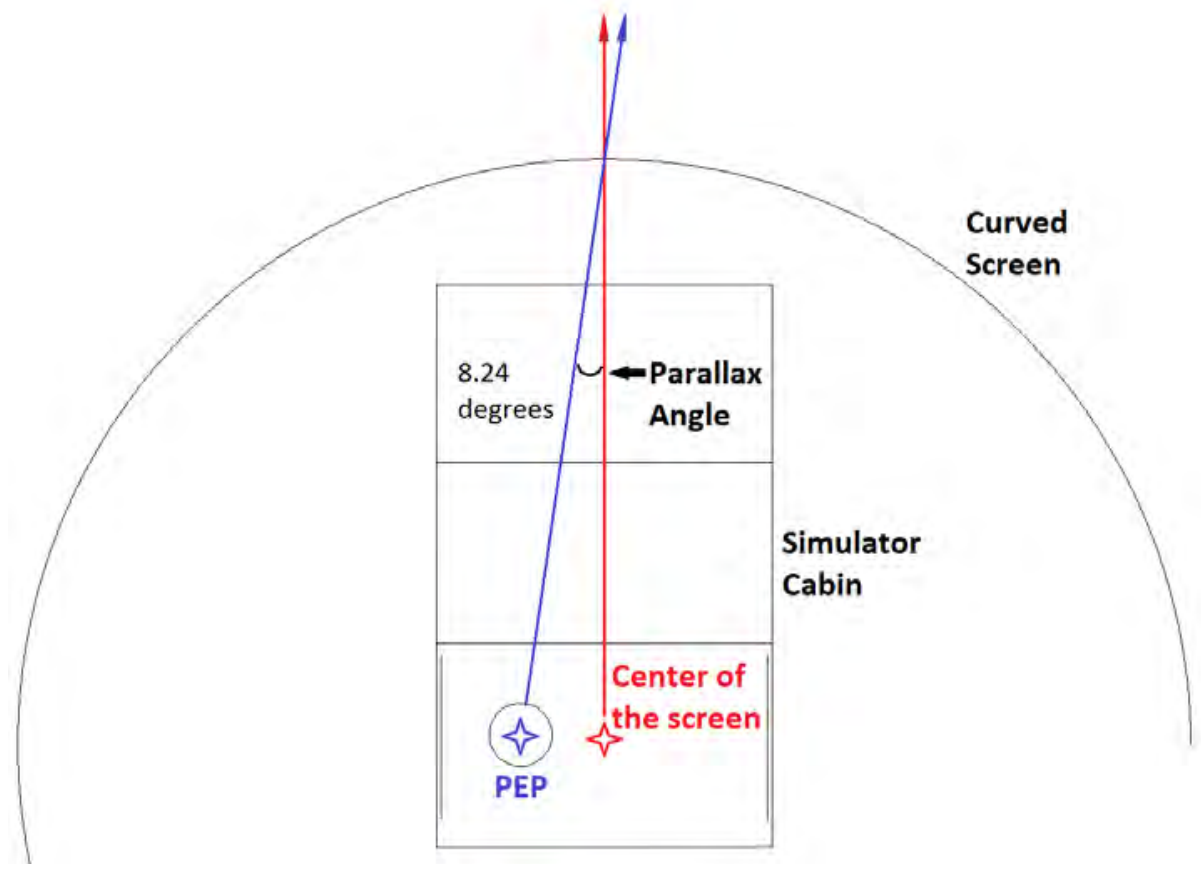

Figure 2.45: Parallax angle of the CURVS VCS which is 8.24 degrees. 


\section{Chapter 3}

\section{Vibratory and Auditory Cueing}

In this chapter the design and development of both auditory and vibratory cueing systems for the CURVS are discussed. The input source for both sound and vibration are the same and that is the engine noise. Though, they are considered as separate cueing systems as they are perceived as different cues by humans.

\subsection{Design}

The design of the sound and vibration cueing systems commenced with investigations about sound and vibration in flight tests and then proceeded with the design of systems for implementing the required cues using primarily COTS components. For the sound system, in a C172 while the engine is running it is almost impossible to talk without using headphones. However, in the FSTDs experienced during the investigations (Alsim at WWFC; Mechtronix at OAS; and also the VectorSim prior to upgrading, see Figure 3.1) only a low power stereo sound system was found to be implemented. That is because in most of the FSTD hours that students fly, an instructor sits close to the student and talks to him/her throughout the flight. Therefore, the flight instructors tend to reduce the sound volume to be able to give instruction to the students without using intercom-equipped headphones. The drawback of using 
low-power small speakers in the FSTD is that the student cannot experience the immersive loud engine noise that is experienced in the actual aircraft, especially when he/she is practicing solo. Therefore, based on the personal experiences described, it was decided to implement a sound system that features the option of having a loud sound experience.

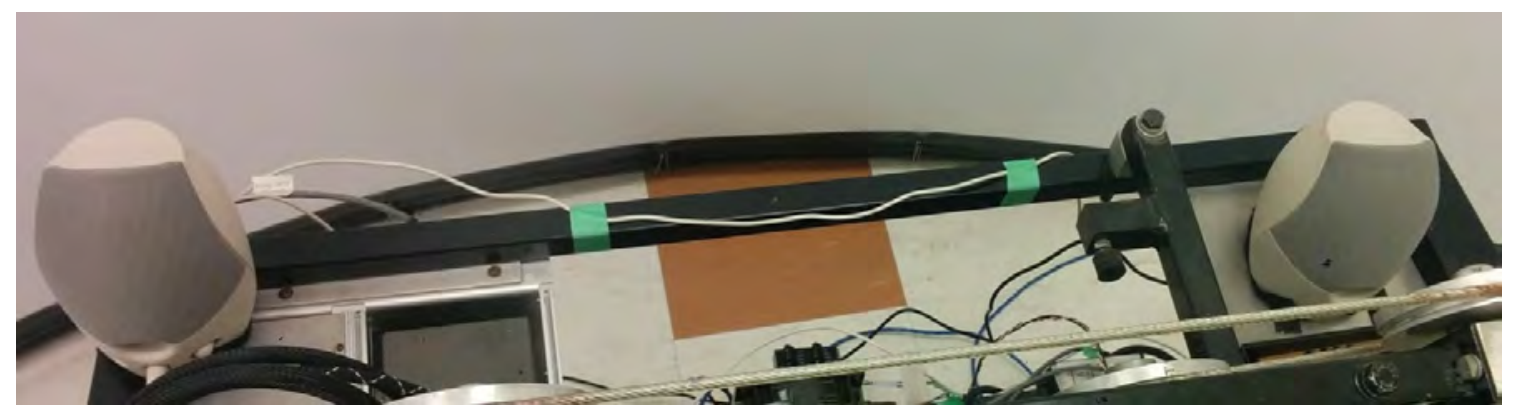

Figure 3.1: Dual channel stereo speaker previously used in the VectorSim.

The vibration during the flight tests was also found to contribute to the perception of the flight, particularly during takeoff and touchdown. Vibrations pave the way for the recognition of the transitions between various flight stages. Also, the rotation of the propeller is translated into vibrations to the cabin and thus the pilot can estimate the engine RPM through the stimulation of his/her tactile system.

The cost-effective vibration transducers on the market are tactile transducers or base shakers. A shaker transmits low-frequency vibrations (from sound sources) into various surfaces so that they can be felt by humans. Therefore, having a realistic sound source to generate both high-band frequencies (for the sound system) and lowband frequencies (for the shaker) is important. As such, for the CURVS project, multiple sound files were recorded in the actual aircraft that are discussed in detail in Chapter 4.

The simple diagram in Figure 3.2 illustrates the sound and vibration system implemented in the CURVS. 


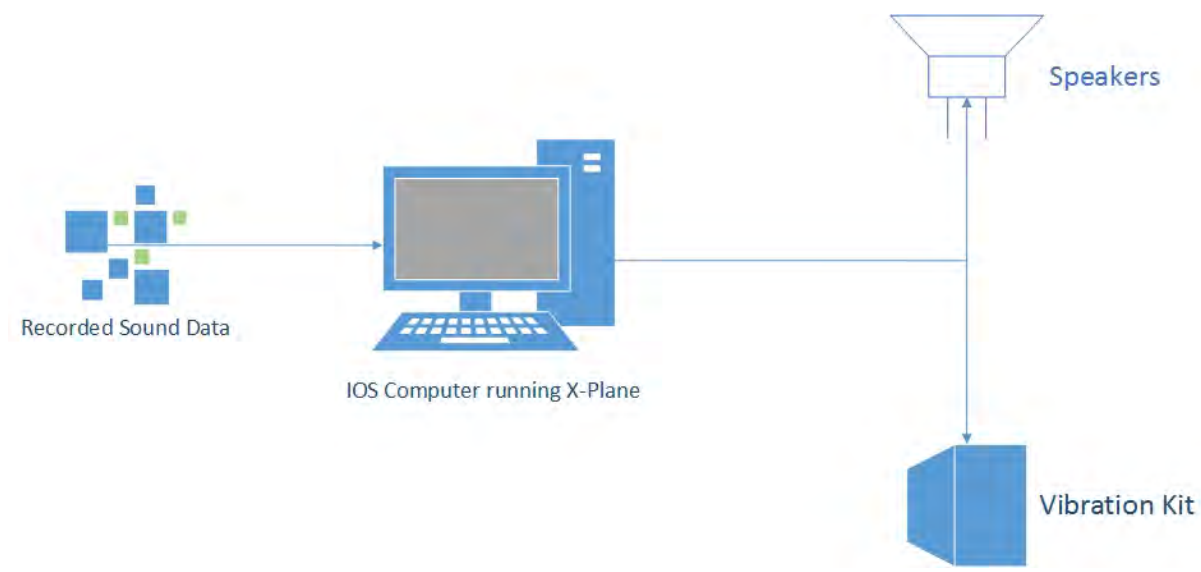

Figure 3.2: Sound and vibration cueing system diagrams in the CURVS.

The vibration transducer that was selected for the CURVS is the Buttkicker package that is shown in Figure 3.3. The idea behind the ButtKicker is to use an audio signal to drive a "low-frequency transducer" comprising a piston suspended in a magnetic coil. As with a normal speaker, an electrical audio signal fluctuates the magnetic field in the coil to drive a piston up and down. But in this case, the piston does not move a diaphragm to create sound waves; it just moves a weight to create vibration based on the base signal that goes to a subwoofer. The Buttkicker also comes with an analogue amplifier that has low- and high-pass cutoff filters that help to optimize the input signals to the desired frequency range.

The speaker system that was chosen for the CURVS is a 5.1 channel sound system with 5 speakers and 1 subwoofer having 75 W RMS output power, from Logitech, shown in Figure 3.4. The system has the ability to adjust the volume (sound intensity) and base intensity. Albeit, X-Plane is not currently inherently capable of producing six channel sound output from multiple sources. The 5.1 channel sound system, however, is advantageous as it generates an immersive multi-directional sound environment. 


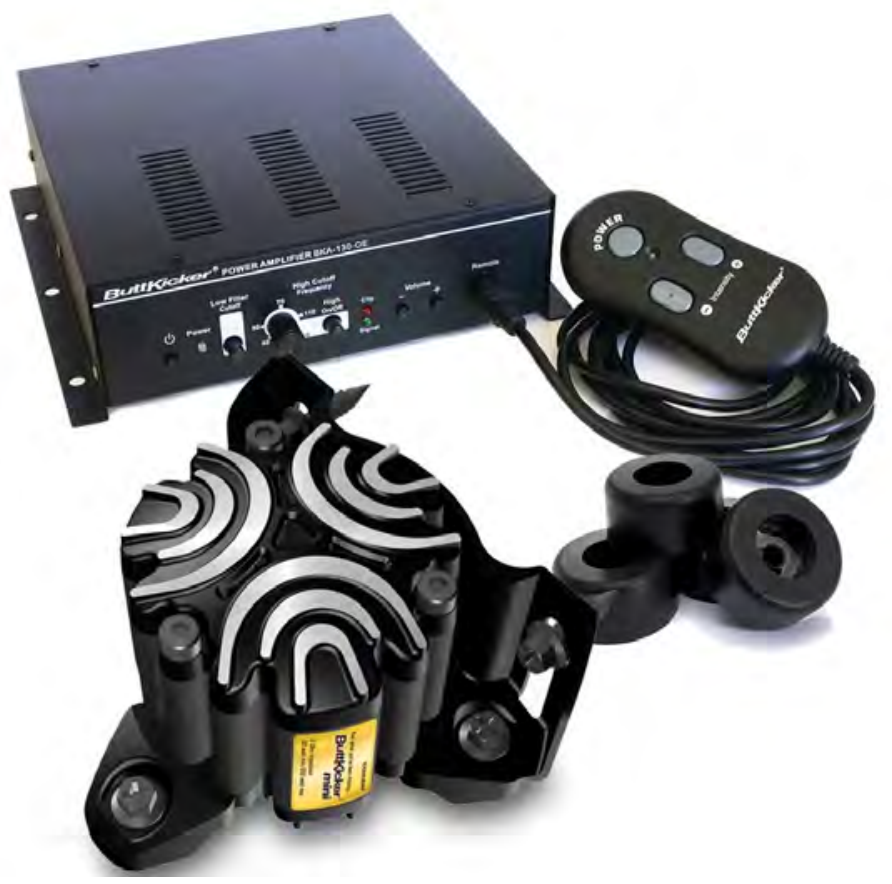

Figure 3.3: The Buttkicker package.

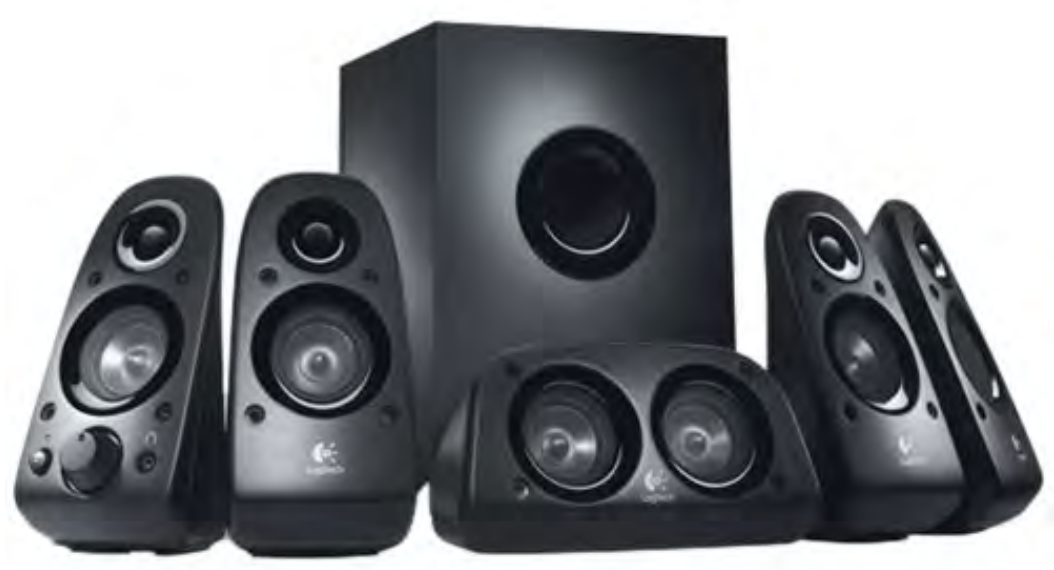

Figure 3.4: The sound system for the CURVS. 


\subsection{Equipment Assembly}

Buttkickers are designed in various types for different applications. The type that was utilized in the CURVS is specifically for simulators. The simulator version of the Buttkicker has an amplifier that can be used to eliminate noise and tune the vibrations to the desired low-frequency components.

The vibrator unit of the simulator version of the Buttkicker comes with screws and and holes making it easy to attach to the seat. It was decided to put two units into effect, one for each of the pilot and co-pilot seats. After evaluating the plausible locations to attach the seat shakers, it was decided to install the units under the seats. The location also provided access to pass the wires from behind the seats where the amplifiers were to be located.

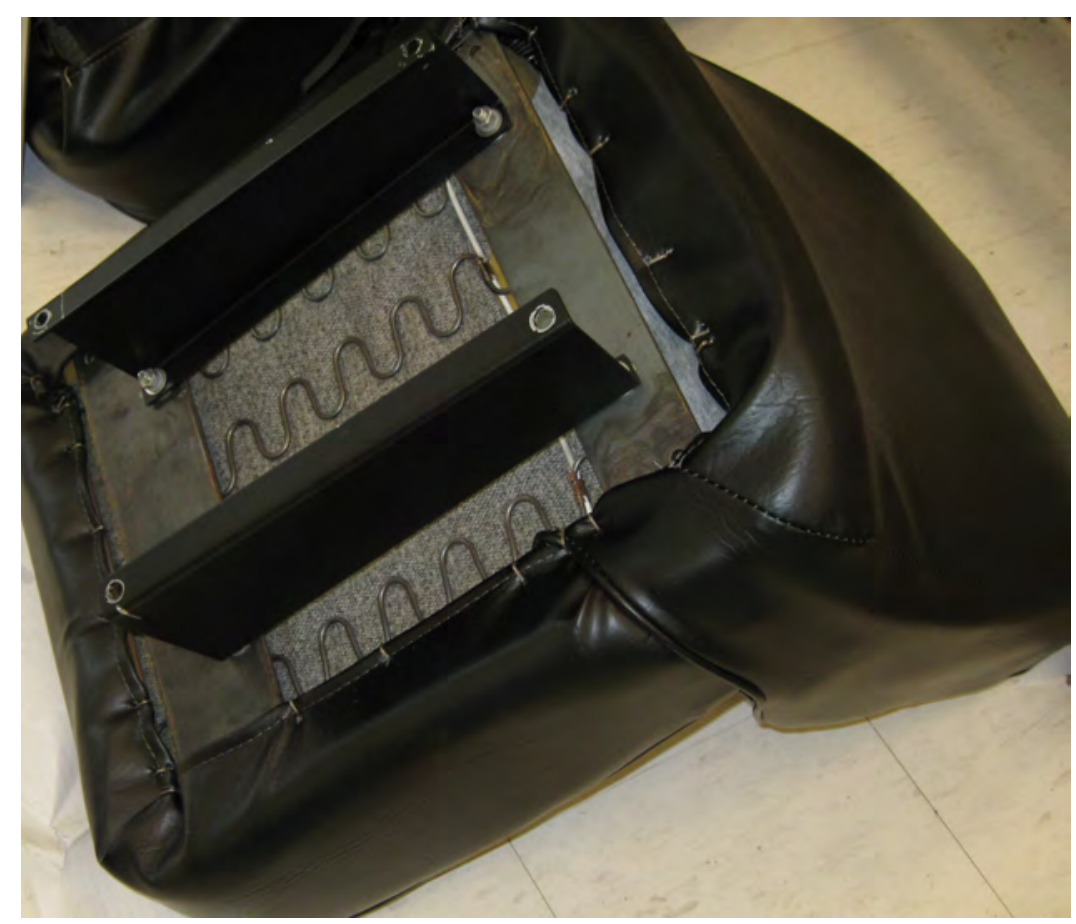

Figure 3.5: The pilot seat detached from the cabin.

The seats from the original VectorSim are connected to metal components that can slide and make the seat adjustable backward and forward. However, there was 
not any specific place to attach the units; therefore, a customized metal interface plate for the units was required. Figure 3.7 illustrates the geometry of the aluminum plate that was fabricated in the Mechanical and Aerospace Engineering machineshop. The plate and its find installation are shown in Figure 3.6. Moreover, in order to isolate the vibrations from the other parts of the cabin, and the instrument panel specifically, rubber isolators were also installed between the seat assembly and the simulator frame. The isolator utilized is shown in Figure 3.8 and the final assembly in Figure 3.9.

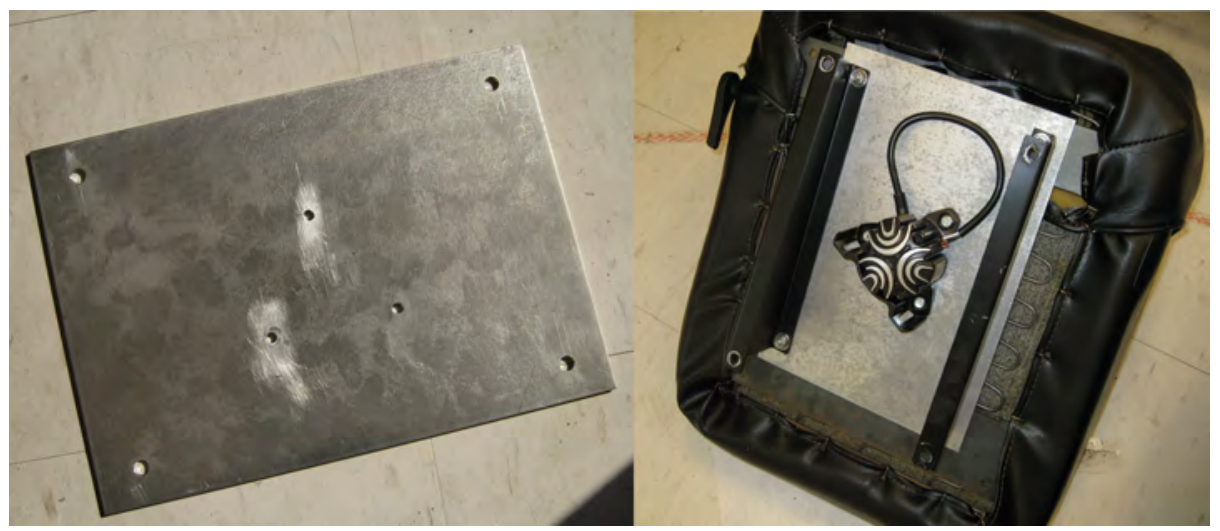

Figure 3.6: The aluminum plate that was built for the Buttkickers on the left and then attached to the seat on the right.

The speakers were located and tested before finding a final location for each of the individual channels by locating them around the cabin shell. As X-Plane cannot generate multiple-channel sound, the order of each individual speaker did not matter. Therefore, an attempt was made to surround them symmetrically to produce an enveloping sound environment. Figure 3.10 shows the preliminary tests for the sound system assembly. The final speaker placement has been deferred until the exact placement of all components in the overall simulator project has been finalized. 


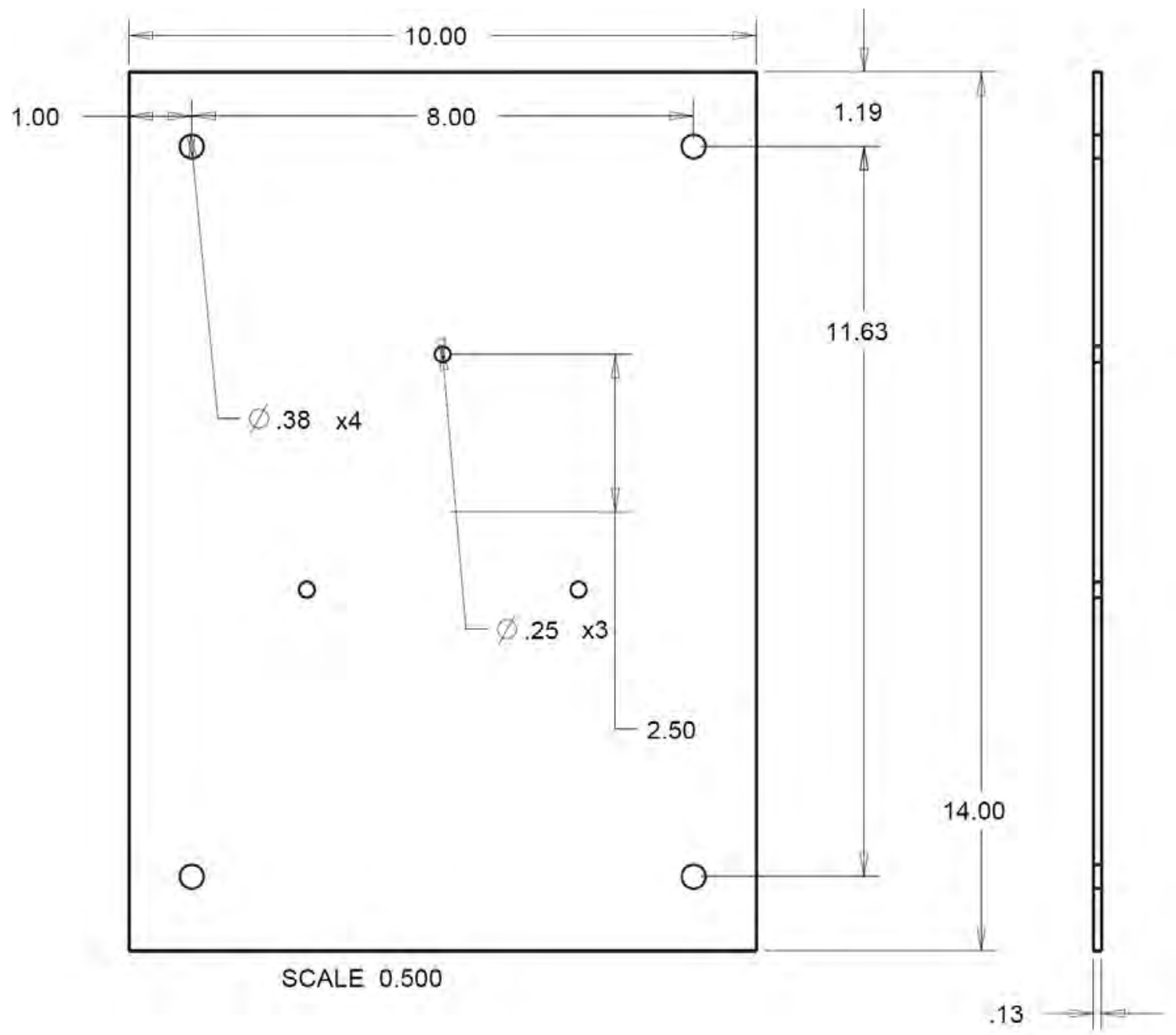

Figure 3.7: The metal plate that was drawn for the Buttkickers attachment (all the units are in inches).

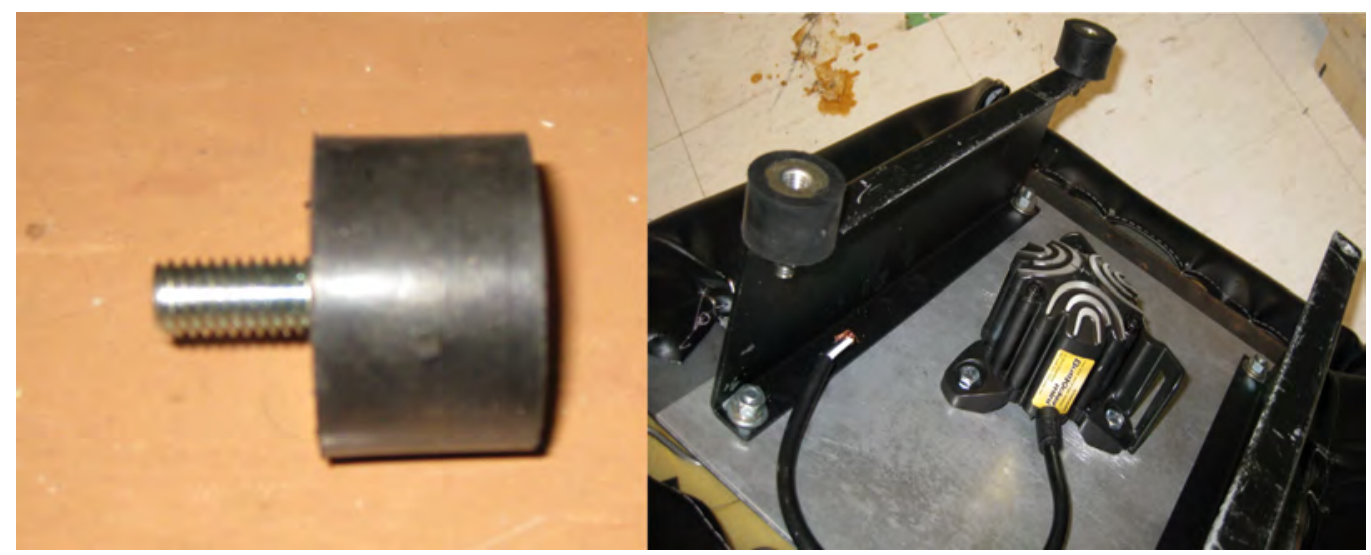

Figure 3.8: The rubber isolators on the left and attached to the seat on the right. 


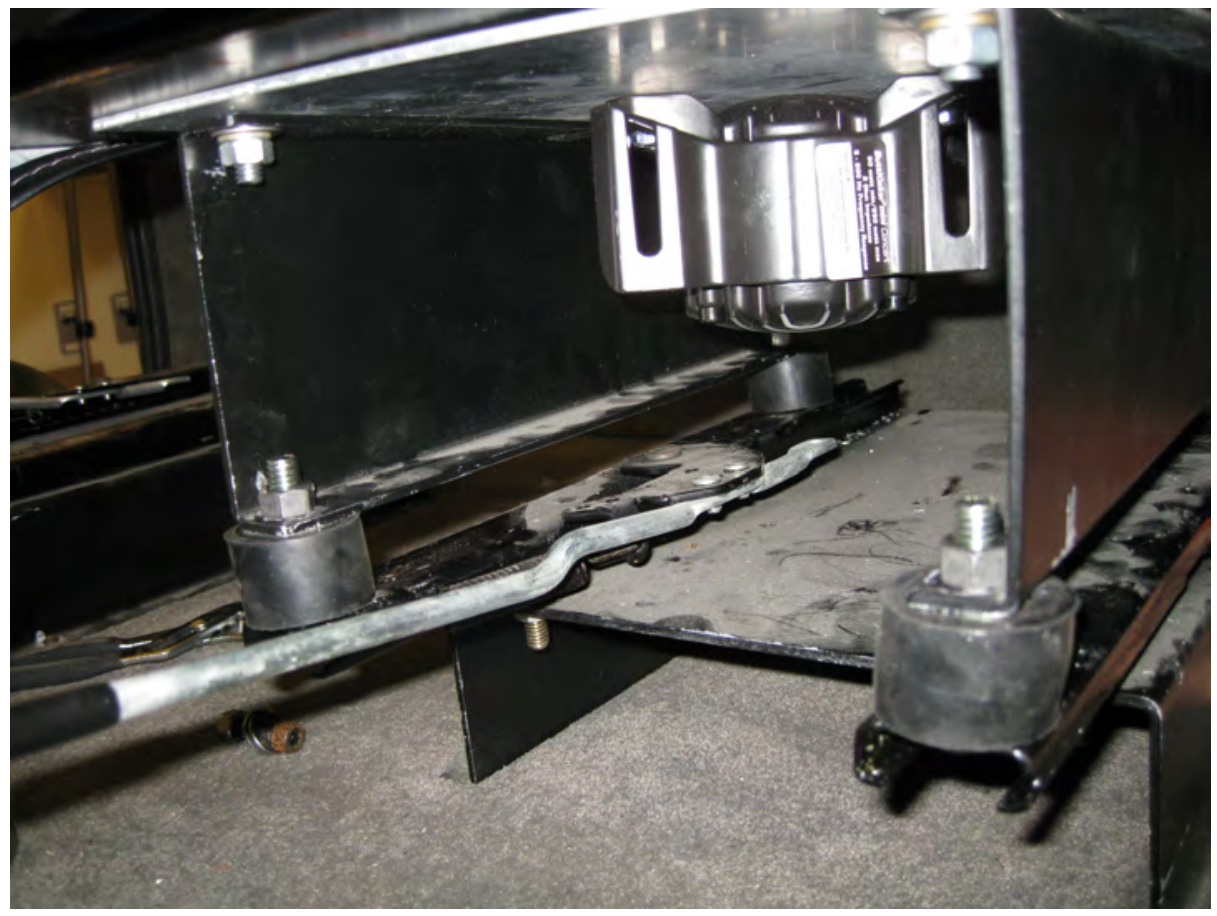

Figure 3.9: The upgraded seat re-attached to the simulator cabin.

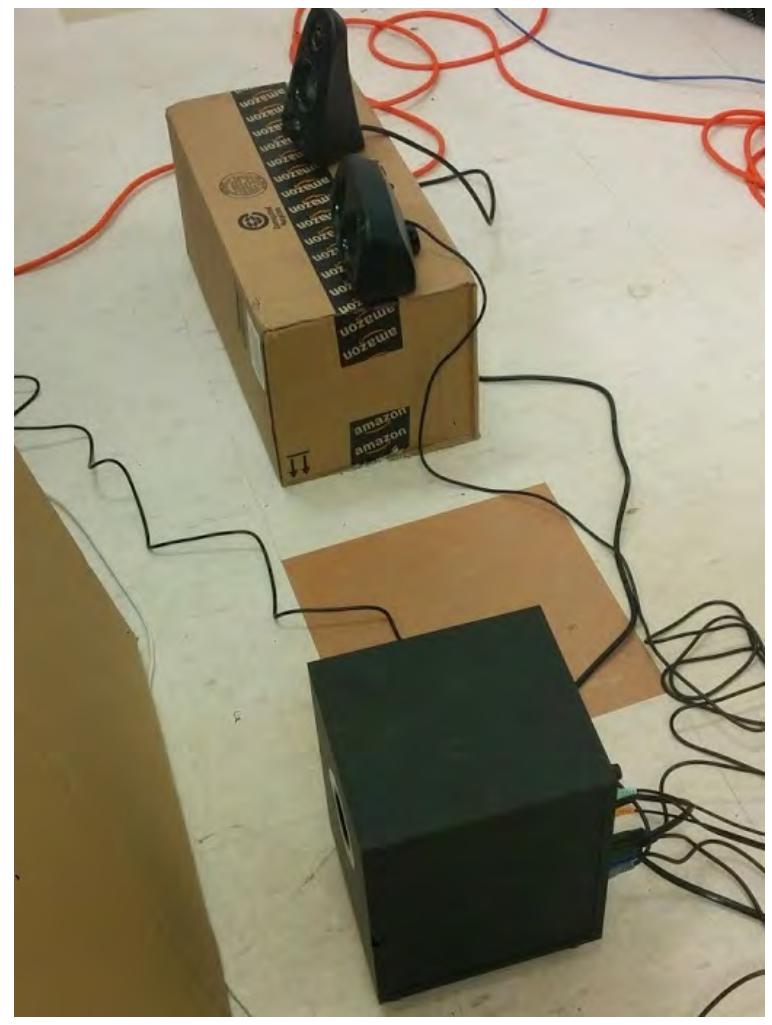

Figure 3.10: The 5.1 channel surround sound system. 


\subsection{Sound Tuning}

X-Plane provides the option to modify the sound of the aircraft to that desired by recording sound files and indicating the engine RPM at which the sound was recorded. The sound files have to be in waveform audio format (WAV) with a minimum data point sample rate of $22.050 \mathrm{~Hz}$. X-Plane applies its own algorithms to change the pitch (frequency) and magnitude of the sound in response to constantly-varying engine RPMs and airspeeds in real-time. Unfortunately, no published document was found that fully describes how exactly X-Plane manipulates the sound. Nevertheless, using spectral analysis, in the next section, it was possible to compare its output to the actual aircraft.

There were multiple sound files recorded in the flight tests (as explained subsequently in Chapter 4) with a variety of engine RPMs. Normally, when the engine is at idle in a $\mathrm{C} 172$ the engine runs at $750 \mathrm{RPM}$. Also, as the propeller rotation rate increases as a result of increasing engine RPM, the wind speed induced over the fuselage increases the wind shear on the aircraft body which correspondingly generates more wind noise. Therefore, the lower the engine RPM during the recordings, the less noisy are the sound files that are acquired. X-Plane generates the wind noise from another file source and as such desirably the engine sound recording must include only the engine noise components without the the components induced by the wind.

Finally, as the engine sound levels at higher RPMs were higher than for lower RPMs, a sample file at 1700 RPM was chosen to be implemented into X-Plane by using Plane Maker, an aircraft editor software that comes with X-Plane, as shown in Figure 3.11. The sound was recorded while the aircraft was on the ground with the parking brakes on and the engine was throttled up to 1700 RPM. This number is also

close to the middle of the $\mathrm{C} 172$ effective engine RPM range and thus thought to be the most appropriate sound recording sample. 
Apart from the engine noise, the electrical flap deflectors generate a sound which was found to be important for C172 pilots. The flap motor sound is an indication that flaps are being deflected as intended when the pilot sets the flap handle. However, the aircraft on which the flight tests were performed did not have an electric flap deflector. Therefore, no sound recordings were available for the flaps and the original generic X-Plane sound files were implemented.

When it came to the gyros, the original generic avionic sound in X-Plane was so unrealistic that it definitely needed to be replaced. Therefore, the gyro (avionics) sound was also recorded when the aircraft was off and only the battery and master switches were on.

Before implementation, the recorded sound files needed to be modified using sound-modifying software in three stages. First, the sample files had to be cut out from long recorded sound files. Second, in order to experience a seamless simulated sound, X-Plane requires a perfectly-looped sample file (10 seconds for instance) meaning the start and the end parts of the files have to be connected at zero data points. And thirdly, as both flaps and avionics sounds were found to be not adequately powerful in the simulator, they needed to be amplified. All three modifications were made using the Audacity software package 3.12. [51] 


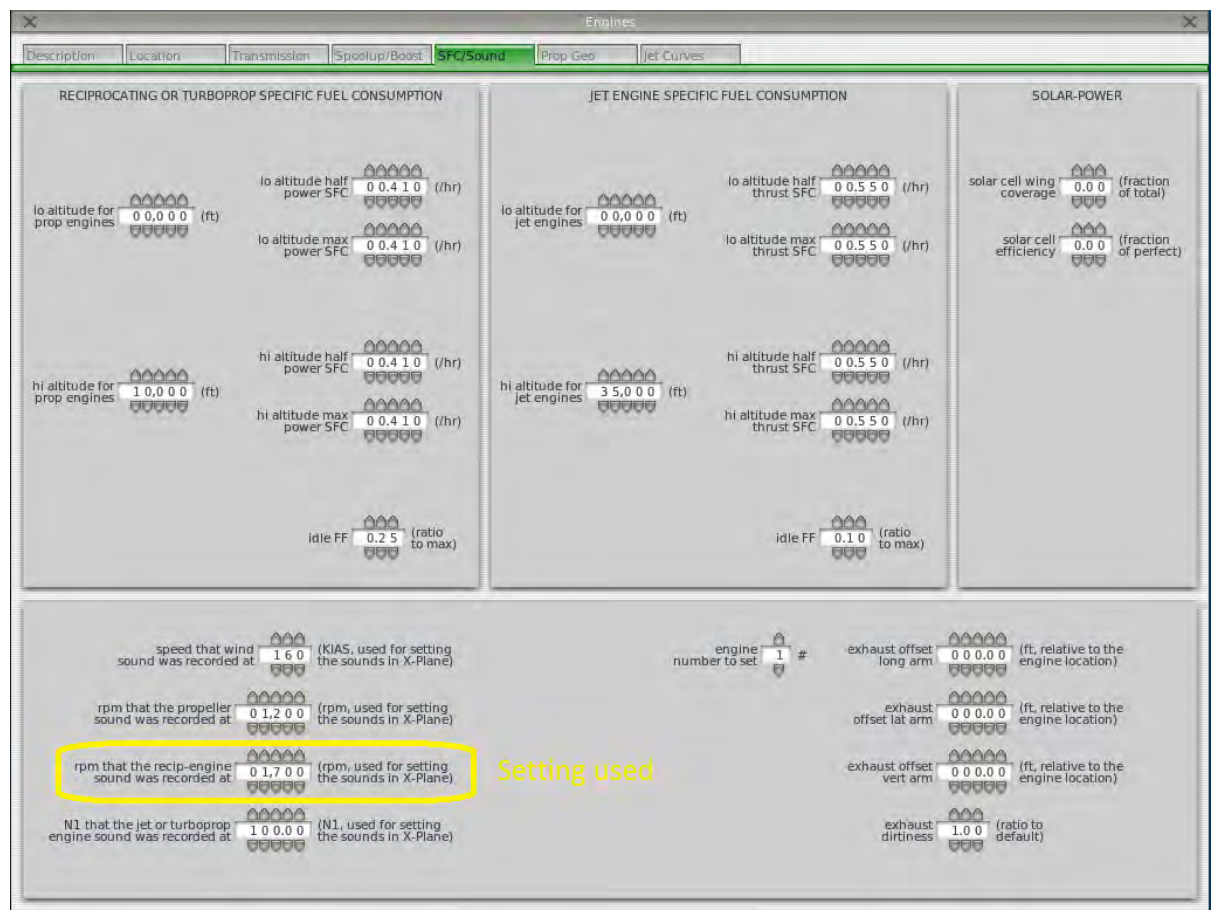

Figure 3.11: Plane-maker settings for the sound files.

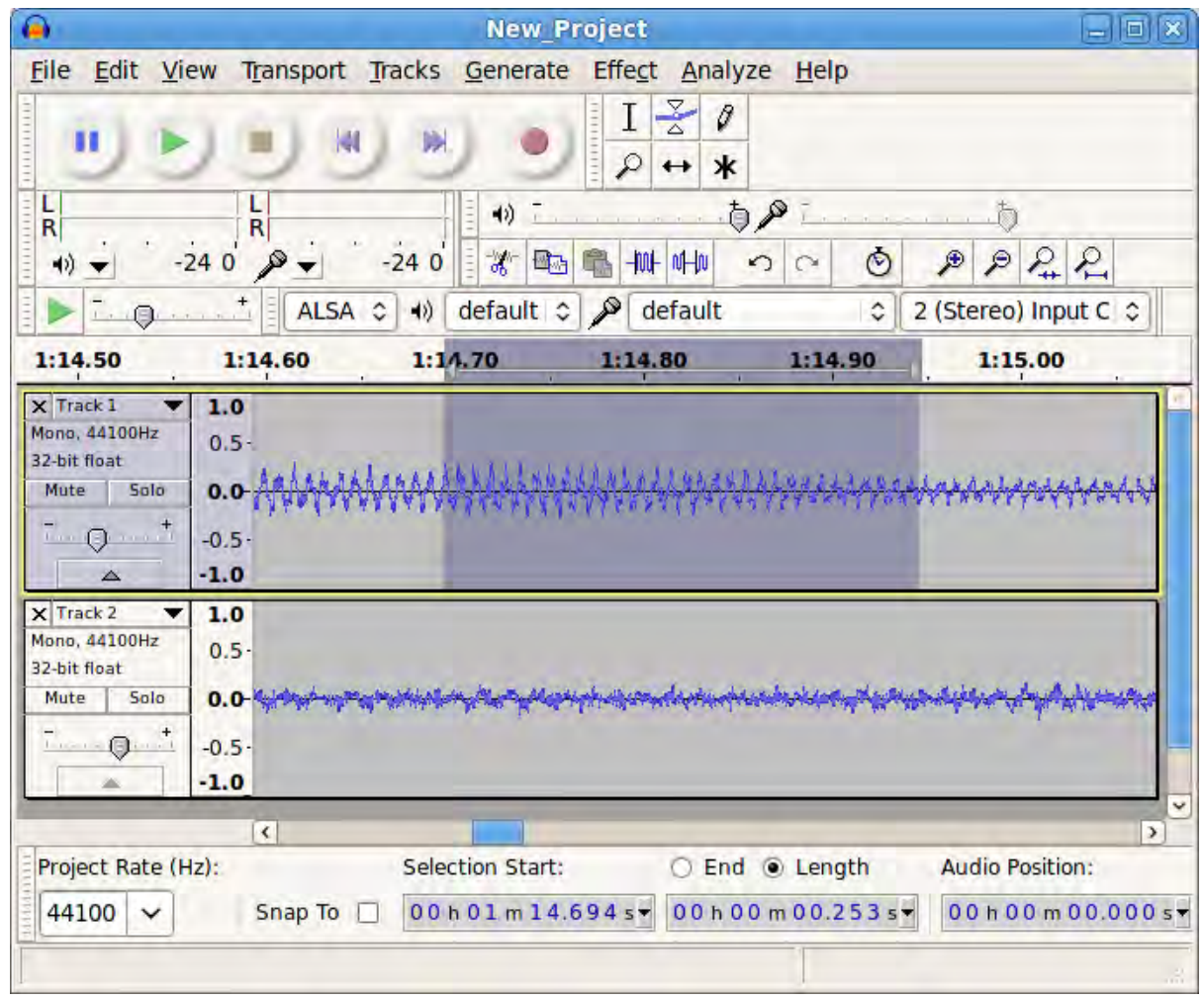

Figure 3.12: The Audacity interface. 


\section{Chapter 4}

\section{Experiment and Analysis}

In this chapter, two different types of tests conducted for the CURVS project are presented. Firstly, flight tests were performed to collect quantitative data for the CURVS simulator design and tuning and secondly, after the simulator was built, multiple simulator flight tests were performed to evaluate the output of the CURVS simulator qualitatively and quantitatively.

\subsection{Cessna 172 Tests}

Three separate flights were performed in a C172 for various reasons. The first two flights were carried out in a private C172 aircraft flown by Chris Hobbs, an instructor pilot at the Rockcliffe Flying Club based at the Rockckcliffe Airport (CYRO) in Ottawa, Ontario, Canada. The third one was in another C172 during a flight for a pilot who was filling hours during his night flight training sessions in Ottawa/Macdonald Cartier International Airport (CYOW) in Ottawa, Ontario, Canada. These flights were not officially required to obtain flight test permits from Transport Canada as there was no equipment on board that could endanger the aircraft safety. However, during each flight, different data, sufficient for this research, were collected and recorded to be applied through the design and tuning processes of the simulator as 
described below ${ }^{1}$.

\subsubsection{First Flight}

The first flight was flown on June 7, 2015, a warm humid day, at CYRO during which pilot view and acceleration data were collected. In order to distinguish the spots the pilot looks at most during different manoeuvres, a GoPro camera was attached to the pilot's head and the flight was recorded as video, see Figures 4.1 and 4.2.

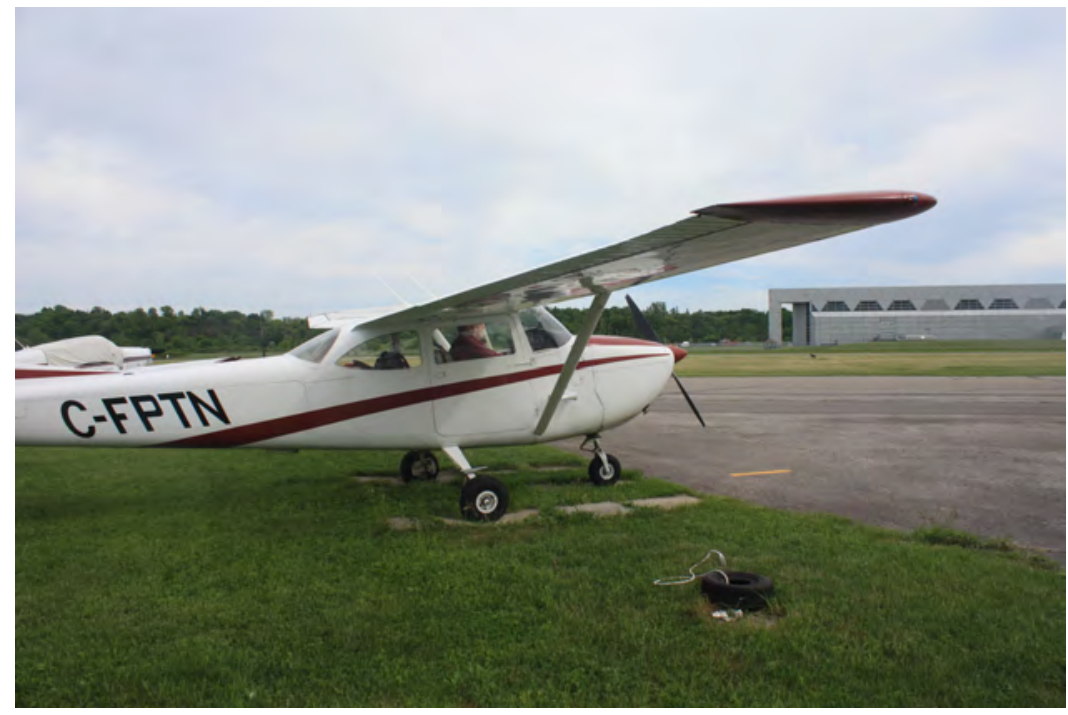

Figure 4.1: The Cessna 172 flown for the flight test.

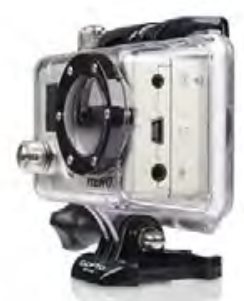

Figure 4.2: A GoPro Hero 2 camera attached to the pilot's head.

\footnotetext{
${ }^{1}$ The METARs for weather data of the days of the flight tests are provided in Appendix D. Also, the specifications for the instruments used are provided Appendix E
} 
Although by using a GoPro camera it was not possible to track eye movement and thus the specific eye focal point, it gave a preparatory understanding of the horizontal and vertical FOV that is needed for the student pilot to practice the various stages of flight. The other rationale behind this test was to examine the necessity of an overshoulder rear view during a circuit and to appraise the horizontal FOV needed for the FSTD. Having attached the audio input of the camera to the aircraft's communication radio, instead of engine noise, all the communications during the flight were recorded in the video. This paved the way to recognize the manoeuvres more easily for further analysis.

Moreover, multiple pictures were taken using a handheld camera as well as a panoramic view of the cockpit to get a comprehensive view of a C172 cockpit throughout the flight, as illustrated in Figure 4.3.

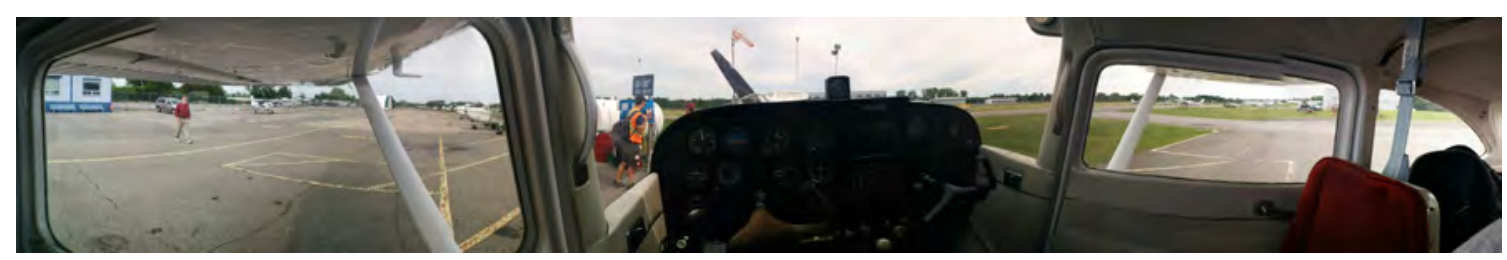

Figure 4.3: Panoramic view of a C172 cockpit.

The flight started by taking off from Runway 09, flying at 2500 feet over the Gatineau River, and performing ab-initio practice such as flying level, approaching the Gatineau Airport, and carrying out touch-downs followed by two circuits. During the flying level practice, the students are told to check if they see $1 / 3$ ground and $2 / 3$ sky vertically in the forward direction. This condition had to be implemented subsequently and satisfied in the CURVS VCS. After the last touch and go at the Gatineau airport we headed back over North-east Ottawa and passed over the Rockcliffe airport. When pilots get over the airport they usually report their position and acknowledge that they are passing over the airport; and this is called an over-the-field (OTF) report. In order to do that in a $\mathrm{C} 172$, the rule is to look out the side window 
and spot the aircraft's gear. Whenever the airport appears to be blocked by or close to the gear it means that the aircraft is right above the airport, as shown in Figure 4.4. This needs the simulator to have a significantly wide VFOV that would require projectors to be arranged in columns, and thus was not implemented in CURVS VCS.

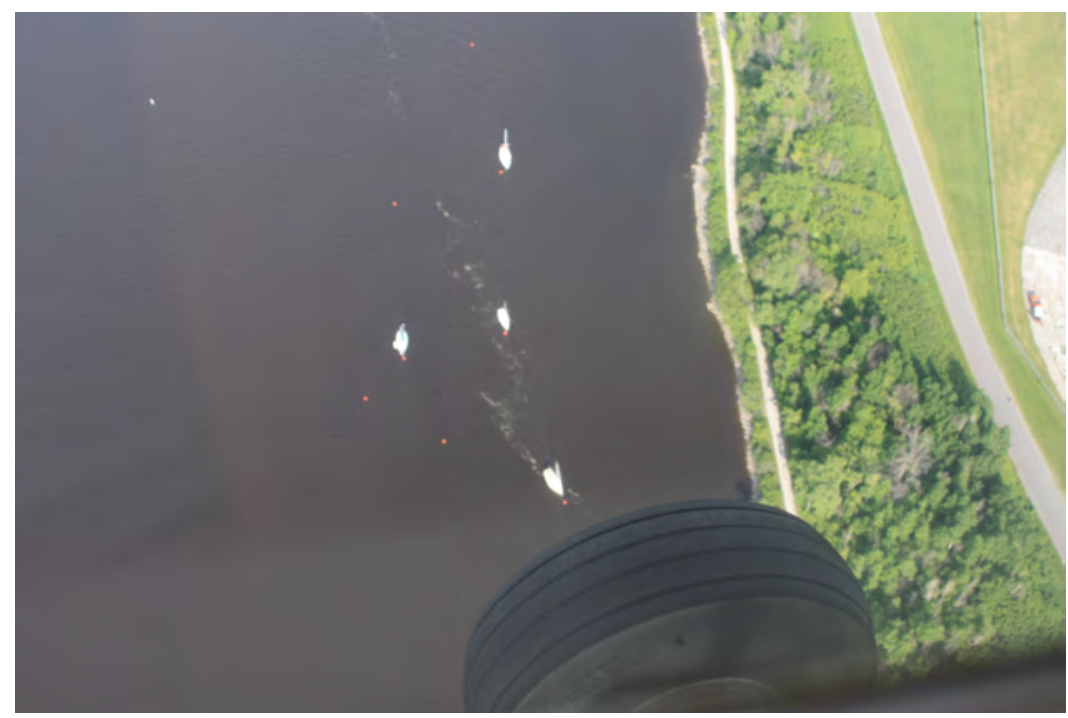

Figure 4.4: Gear view of the C172.

\subsubsection{Second Flight}

The second flight test was performed on August 8, 2015 with the same aircraft this time to record acceleration data, engine noise, engine sound pressure level (SPL), and ambient light intensity. This flight test was conducted with the help of Brent Cameron, another team member on the CURVS project.

The acceleration (ACC) of the pilot seat was recorded using a portable accelerometer as shown in Figure 4.5. The data is stored in 'Comma Separated Values' (CSV) file format that was exploited for 'frequency analysis' using Matlab software. Although ACC data of the whole flight was collected, as the sampling frequency of the device was set to only $50 \mathrm{~Hz}$, the sampling frequency was found to be too low. 
Therefore, ACC data analysis was deferred to a subsequent flight test when a higher sampling rate could be used.

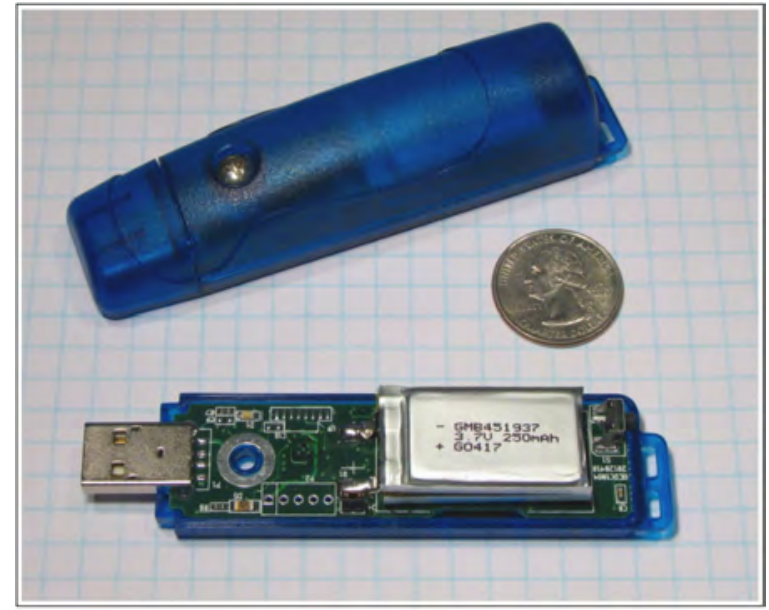

Figure 4.5: Gulf Coast Data Concepts X16-2 portable USB accelerometer.

The engine noise was recorded using a stereo sound recorder, shown in Figure 4.6, at a sample rate of $44.1 \mathrm{kHz}$. Multiple files in Waveform Audio File Format (WAV) were recorded for each stage of the flight with various engine RPMs. In order to mark what was being recorded, before starting to record, a brief explanation was spoken into the microphones in each recording file.

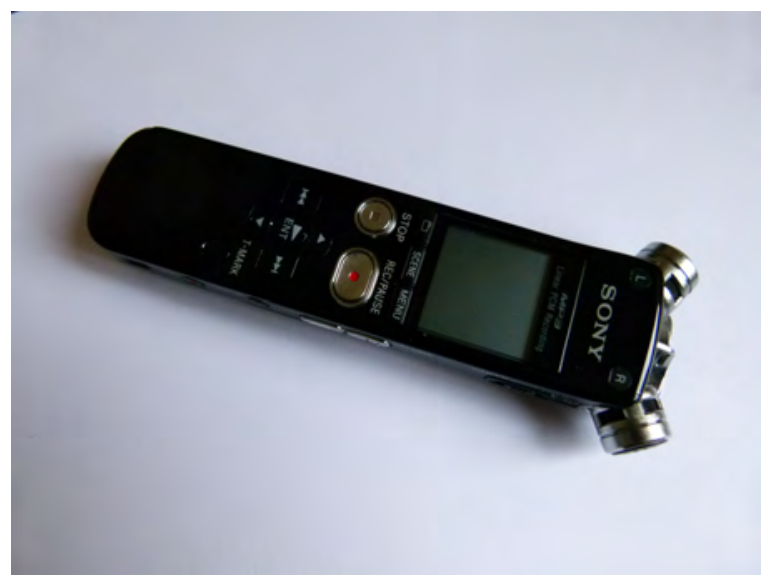

Figure 4.6: Sony digital audio recorder that was used to record engine noise.

The maximum SPL was also manually recorded any time that the sound was 


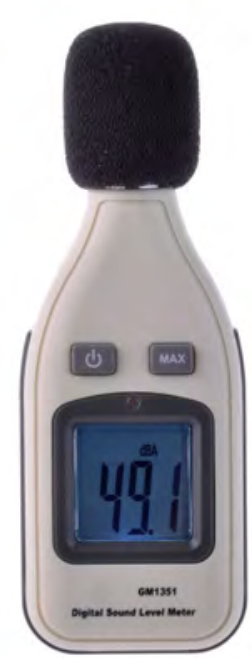

Figure 4.7: Digital sound pressure level meter.

being recorded using an SPL meter, as demonstrated in Figure 4.7. The SPL meter measures and indicates the SPL of the ambient noise at $2 \mathrm{~Hz}$ and has the capability to save the maximum value that has been captured. Using this feature, the maximum SPL of each flight manoeuvre was written down. The only drawback of this sound level meter was that its frequency response was between 31.5 - $8500 \mathrm{~Hz}$ meaning that it cannot show the very low frequency range.

This flight was a short trip with multiple manoeuvres including taxing, takeoff, flying level, approach, and landing to the airport, all of which were flown in the proximity of CYRO. The flight was also recorded on video by the GoPro camera this time attached to the top of the aircraft cockpit. Having the whole flight recorded on camera, it was easier to analyze the flight afterwards.

In addition, light intensity of the environment was measured during taxi, takeoff, cruise, and on the final leg using the spot meter shown in Figure 4.8. The unit of measure in which the spot meter indicates the brightness is in exposure value $(\mathrm{EV})$. 


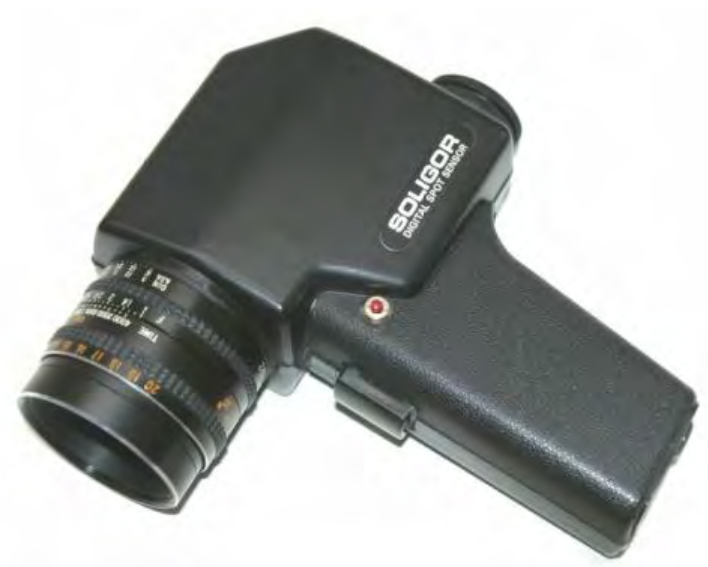

Figure 4.8: Spot meter that measures light intensity in EV.

\subsubsection{Third Flight}

The last flight was offered by a student pilot who was attempting to fill flying hours for his NR license. The flight was at night and was a good opportunity to evaluate how night flights are performed in a C172. The flight started from CYOW and proceeded to the Gatinuea Airport around which multiple circuits were practiced; and was ended by flying back over Ottawa and landing on Runway 14 at CYOW as shown in Figure 4.9. During this flight, seat acceleration data was also recorded by attaching the portable accelerometer to the co-pilot seat. It was assumed that there is no difference between pilot and co-pilot seats in terms of vibration as they have the same but mirrored placement relative to the engine.

The vibration data was recorded at $400 \mathrm{~Hz}$ meaning that the spectrum analysis could be performed up to $200 \mathrm{~Hz}$ (Nyquist frequency of the data). Figure 4.10 illustrates the orientation of the accelerometer during all the flight tests. Moreover, the flight was captured on video by the GoPro camera attached to the rear window of the aircraft; however, the resulting footage was not useful as it was too dark to evaluate the video. 


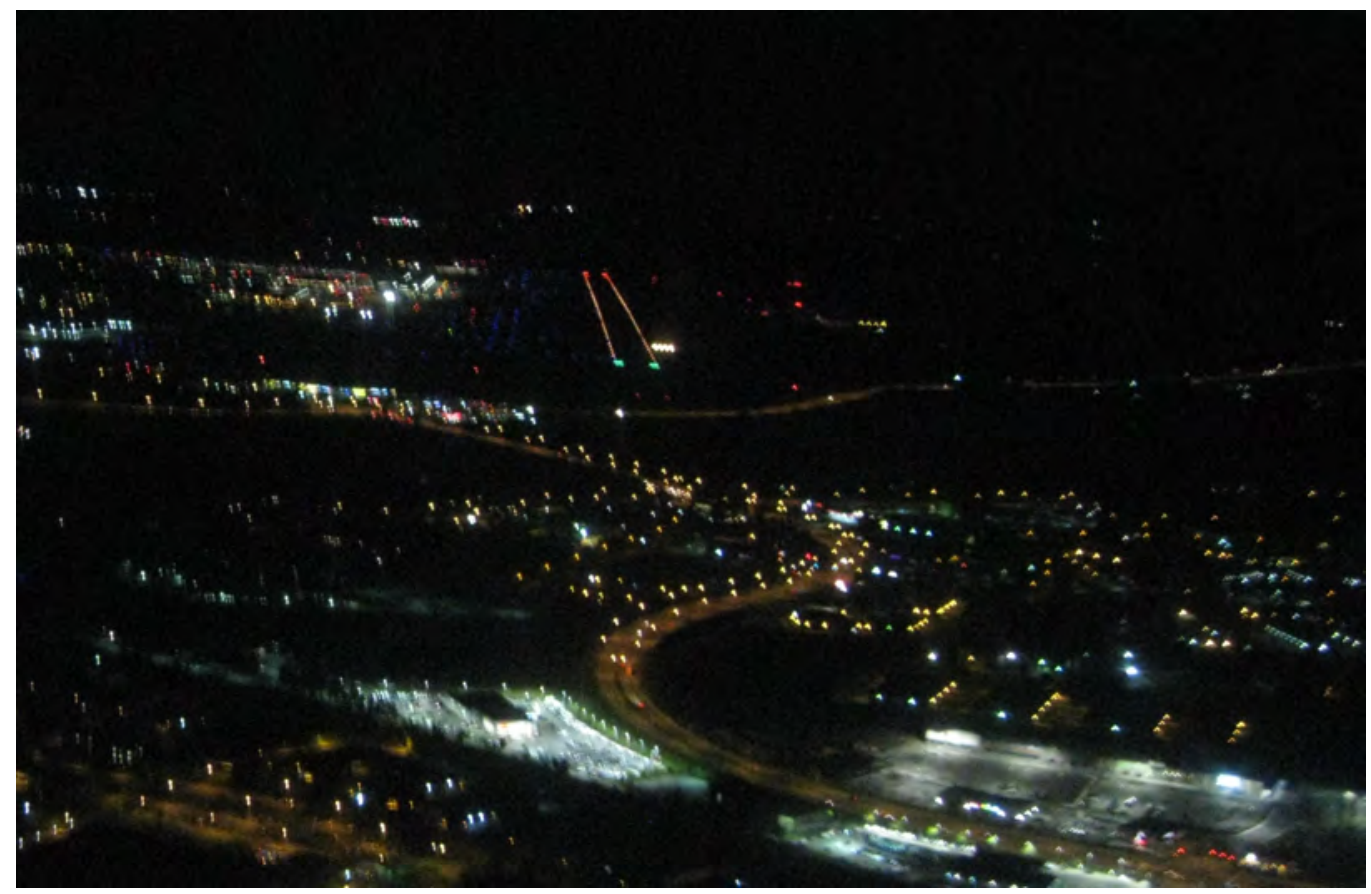

Figure 4.9: Night flight over Ottawa, approaching on Runway 14 at CYOW.
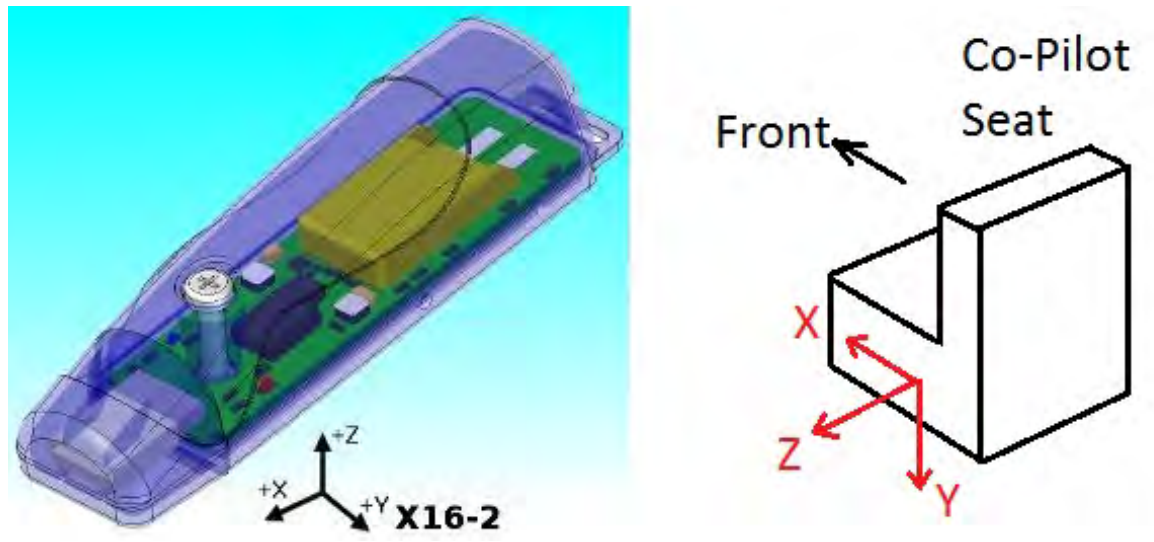

Figure 4.10: The accelerometer's axis and its orientation in the test. 


\subsubsection{Simulator Quantitative Test}

The simulator testing consisted of two parts: a qualitative test and a quantitative test. The quantitative test was similar to the actual aircraft test discussed in the previous sections. The qualitative test was to evaluate the simulator cueing systems and get feedback from actual pilots and personal opinions about their simulation experience using the CURVS. The qualitative test is described in last section of this chapter.

In the quantitative test, acceleration data, SPL, engine noise, and also screen brightness were examined using the same tools utilized in the actual aircraft. The accelerometer was attached to the pilot seat to record vibrations induced by the seat shaker. Engine noise provided by the sound system was recorded using the same voice recorder and the maximum possible SPL was measured by the SPL meter. Also, the brightness of the screen was measured using the spot meter. The resulting data were analyzed and are discussed in the following section. 


\section{$4.2 \quad$ Results}

\subsubsection{Summary of the Tests}

All the flight tests are briefly summarized in Table 4.1.

Table 4.1: Summary of flight tests

\begin{tabular}{|c|c|c|}
\hline & Objectives & Result \\
\hline Flight Test 1 & -Field of view evaluation & $\begin{array}{l}\text {-At least } 220^{\circ} \mathrm{HFOV} \text { and sufficient } \\
\text { VFOV to see } 1 / 3 \text { ground and } 2 / 3 \text { sky } \\
\text {-OSV for circuits particularly on the } \\
\text { pilot side } \\
\text {-OTF report }\end{array}$ \\
\hline Flight Test 2 & $\begin{array}{l}\text {-Sound recording } \\
\text {-Seat Vibration Data } \\
\text {-Sound pressure level } \\
\text {-Light intensity }\end{array}$ & $\begin{array}{l}\text {-The sound files were recorded on a } \\
\text { voice recorder } \\
\text {-Acceleration data was recorded at } \\
50 \mathrm{~Hz} \text { that was not a sufficient } \\
\text { sampling rate } \\
\text {-SPL was measured by the SPL meter } \\
\text {-Light intensity was captured by a } \\
\text { spot meter }\end{array}$ \\
\hline Flight Test 3 & $\begin{array}{l}\text {-Seat vibration data } \\
\text {-Night flying experience }\end{array}$ & $\begin{array}{l}\text {-Seat vibration was recorded at } \\
400 \mathrm{~Hz}\end{array}$ \\
\hline
\end{tabular}

\subsubsection{Sound Pressure Level}

The SPL varied depending on the manoeuvre and the engine RPM. The recorded values are demonstrated in Table 4.2. The SPL for the simulator in various manoeuvres in comparison to the actual aircraft was also measured. The test was carried out while the speakers surrounded the cabin, approximately where they were planned to 
be positioned in the final version of the flight simulator. The speakers volume was set to $95 \%$ to avoid buzzing noises when the volume is set to maximum. The results are shown in the Table 4.2.

Table 4.2: Sound pressure level in different manoeuvres and engine RPMs for the CURVS and the aircraft

\begin{tabular}{|c|c|c|c|}
\hline Manoeuvre & Engine (RPM) & Aircraft SPL (dBs) & Simulator SPL (dBs) \\
\hline StartUp & N/A & 88.3 & N/A \\
\hline Idle & 750 & 77.8 & 75.1 \\
\hline Engine Runup & 1700 & 92.2 & 78.2 \\
\hline Taxi & 1000 & 81.0 & 72.8 \\
\hline Takeoff & 2100 & 95.2 & 81.9 \\
\hline Cruise & $2300-2400$ & 98.4 & 85.1 \\
\hline Descent & $2000-2100$ & 93.8 & 75.8 \\
\hline Landing & 1100 & 83.4 & 77.9 \\
\hline Gyro Only & N/A & 61.0 & 52.5 \\
\hline
\end{tabular}

When comparing the aircraft and the simulator, the engine idle noises are the closest achieved numbers. Although the aircraft is only $2.7 \mathrm{~dB}$ louder at $750 \mathrm{RPM}$, it means the aircraft is about twice as loud as the simulator. When it comes to cruise, the difference is much greater at about $12.3 \mathrm{dBs}$. This means the aircraft is almost ten times louder than the simulator while cruising. Even the gyro sound that is only a replay of the recorded sound (without any manipulations by X-Plane) is not sufficiently loud.

Although ideally the goal is to replicate the exact sound intensity of the aircraft using the simulator, it might not be beneficial. Firstly, high-quality powerful sound systems are expensive; and secondly powerful sound systems require acoustic isolation of the simulator room which in turn adds to the simulator cost. The final judgment of the simulator sound intensity is discussed in the qualitative test section. 


\subsubsection{Light Intensity}

The day of the flight test was calm but a bit cloudy. The METAR information of the day of the flight is provided in Appendix D. In the aircraft test the spot meter was targeted to the front window once towards the sky and once towards the ground, such as the runway while landing. The results are illustrated in Table 4.3 where all the values are reported in exposure value $(E V)$. The same manoeuvres were performed using the simulator and the resulting light intensity values are compared to the aircraft.

Table 4.3: Brightness level comparison of the simulator and the actual aircraft.

\begin{tabular}{|c|c|c|c|c|}
\cline { 2 - 5 } \multicolumn{1}{c|}{} & \multicolumn{2}{c|}{ Real C172 (EV) } & \multicolumn{2}{c|}{ Simulator (EV) } \\
\cline { 2 - 5 } \multicolumn{1}{c|}{} & Sky & Ground & Sky & Ground \\
\hline Parking & 16 & 14.9 & 6.7 & 5.6 \\
\hline Takeoff & 15.9 & 15.2 & 6.7 & 4.5 \\
\hline Cruise & 16 & 13.8 & 6.8 & 5.0 \\
\hline Final & 15.5 & 14.5 & 6.7 & 4.9 \\
\hline
\end{tabular}

Exposure value is a unit used in light meters that has application mostly in photography. The $E V$ is a number between -4 and 16 that has a corresponding value for luminance L with the SI unit $c d / m^{2}$ (Candela per square metre). An EV value of 3 corresponds to $1 \mathrm{~cd} / \mathrm{m}^{2}$ and every additional $\mathrm{EV}$ is corresponding to a doubling of light intensity, as expressed by Equation 4.1.

$$
L=2^{E V-3}
$$

As shown in Table 4.3, the $E V$ in the outdoor is significantly larger than simulator screen illuminated by the projectors. The TC simulator manual [2] does not mention the requirements for the screen brightness; however, the ICAO simulator manual [31] requires achieving a number of not less than $14 \mathrm{~cd} / \mathrm{m}^{2}$ using a specific test pattern. 
Unfortunately, it wasn't clear how this test could be performed during the current state of the project and therefore it was postponed until closer to when the simulator is planned to be certified.

\subsection{Frequency Analysis}

Human perception in response to various cues is complicated. Pilots may experience a flight in a simulator that is quantitatively different from the aircraft; nevertheless, they assume the flight characteristics are close to the aircraft. In reality, it means that simulators can behave slightly differently than the actual aircraft but the pilots can benefit from the same learning experience as in the actual aircraft. The number of hours that could be transferred from simulated flight to equivalent aircraft flight hours can be calculated using the Equation 1.1, as discussed in Chapter 1. Also in this research, a small-scale quantitative evaluation was performed on the simulator which is described in detail in Chapter 4.

However, it is important to always know how far from reality the simulator behaves. According to the ICAO Manual for FSTDs [31], for vibration and sound, power spectral density (PSD) analysis needs to be performed to demonstrate the cueing system limits of a simulator. PSD analysis is a way to demonstrate the frequency components of a dynamic system (vibration and sound in our case) and the power associated with each frequency. This was done by using Matlab's pwelch function (that applies the Welch method for spectral analysis) to interpret the recorded data from quantitative tests.

The Welch method, that is an improvement on Bartlett's method, divides the data into different segments while allowing $50 \%$ overlapping of the segments as oppose to Bartlett's method which consists of no overlapping. It means that if the data points are divided into $\mathrm{L}$ segments having length $\mathrm{M}$, the number of overlapping points $\mathrm{D}$ is 
$\mathrm{M} / 2$, as demonstrated in Equation. See Figure 4.11. 4.2.

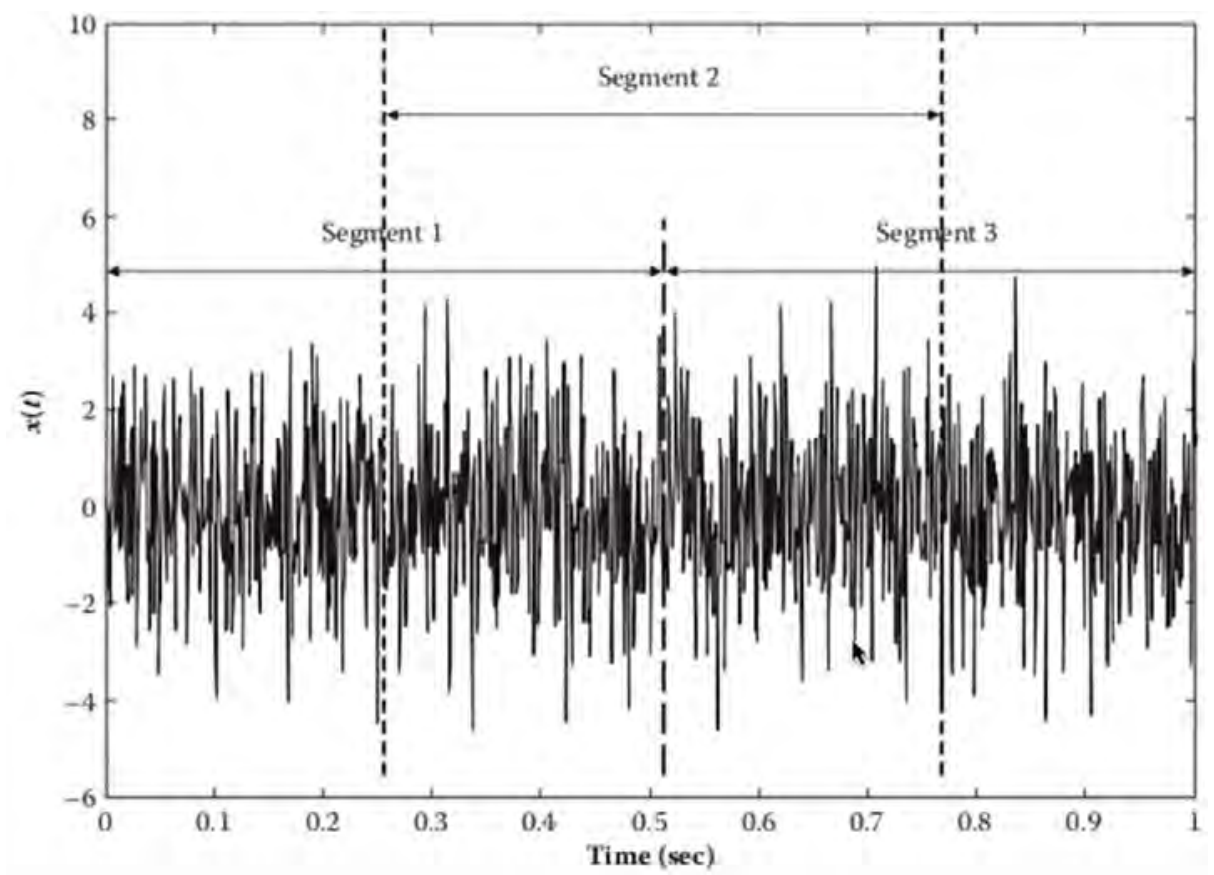

Figure 4.11: Welch method segments with 50\% overlapping of the sequential segments.

$$
D=\frac{M}{2}
$$

The Welch's method inherently reduces the noise of the fluctuations in comparison to other methods (such as Bartlett's method) at the expense of reducing frequency resolution. As we know the oscillations of the aircraft's seats are mostly related to the engine RPM there should be a few discrete peaks in the overall frequency range that are significantly larger than the other frequencies. This is since the only source for oscillations that was recorded on the aircraft was the engine running in the 750-2400 RPM range. Also, while the aircraft was flying the flight manoeuvres should only show as very low frequency peaks and can be eliminated. Therefore, a high resolution in the frequency was not required.

Finally, in order to plot the spectrum using the Welch method, one should take 
the Fast Fourier Transform (FFT) of the data set after the segment divisions. Then by taking the square of the magnitude of the FFT and converting it to the Decibel (dB) scale, the PSD of the data set can be achieved. Fortunately, Matlab has a simple function that calculates the PSD using the Welch method that can be found in Appendix G.

\subsubsection{Vibration Data and Analysis}

The PSD analysis is performed for multiple stages of the flight including engine idle, taxi, cruise, takeoff (on the runway and off the runway), and landing (while in final and also after touchdown). The engine idle is demonstrated and plotted here and other manoeuvres are demonstrated in Appendix F. It is important to note that the accelerometer used for the test had the same orientation for both the simulator and the aircraft as shown in Figure 4.12. However, due to differences in the seat mounts, slight variations in orientation of the sensor may have existed which will be discussed subsequently.

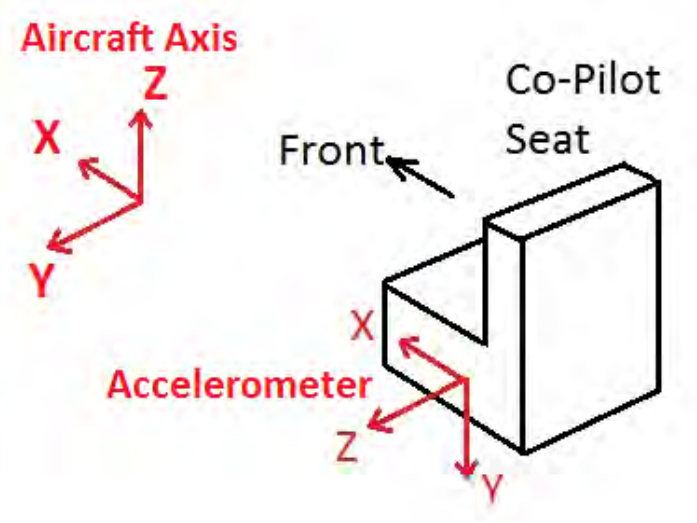

Figure 4.12: Accelerometer orientation.

The data collection was intended to support the analysis within the 0-200 $\mathrm{Hz}$ range corresponding to the frequency response of the Buttkicker. The data collector (the 
accelerometer) was set to record data at $400 \mathrm{~Hz}$ (400 data points per second) such that the maximum achievable signal processing frequency range according to Nyquist theory would be 0-200 Hz, the same as the Buttkicker. However, the accelerometer collected more than 500 data points due to unknown reasons (probably inaccuracy in the device). This was not an issue but made the data noisier. However, thanks to the pwelch function capabilities in Matlab, the noise can easily be reduced by changing the data resolution in peak frequencies. Figures 4.13 to 4.17 demonstrate the analysis when the engine was running at 750 RPM $(12.5 \mathrm{~Hz})$ for both the actual aircraft and the simulator. As illustrated in the spectrum (shown in Figure 4.14), the aircraft seat vibration contains multiple peaks, in all directions, throughout the frequency domain that match in amplitude fairly well except for higher frequencies where the $\mathrm{Y}$ direction (vertical in the aircraft) is larger. This can be interpreted that the vibrations of the seat in all three axes have the same power in the low frequency range.

On the other hand, the spectrum (shown in Figure 4.16) depicts that most of the peaks induced by the Buttkicker are at frequencies below $60 \mathrm{~Hz}$. This is understandable as the Buttkicher's input source is the sound files and not the original vibration signals. However, as is obvious, in different directions the magnitude of the fluctuations varied and ironically the magnitude in the $\mathrm{Y}$ direction is not higher than the other two. Instead, the magnitude in the $\mathrm{Z}$ direction exceeds the other two across the whole frequency range. This was not expected originally as the orientation of the Buttkicker was in the Y direction (Z direction in the aircraft's local coordinate system).

The spectrum illustrated in Figure 4.17 compares the aircraft and simulator vibration in the $\mathrm{Y}$ direction. Note that the magnitude comparison for the two is misleading because firstly, the intensity of the vibrations for the Buttkicker can vary depending on the user's preference; and secondly, the orientations of the accelerometer by which 
the data were recorded were not exactly the same as mentioned previously. Therefore, the Figure 4.17 is only applicable for frequency comparison. As is clear, the strongest frequency component in the simulator is at about $25 \mathrm{~Hz}$ which is relatively close to the $20 \mathrm{~Hz}$ measured in the aircraft. The second strongest frequency in the aircraft is at about $115 \mathrm{~Hz}$ that is not generated by the Buttkicker. The third strongest peak in the aircraft, which is in the range of $38 \mathrm{~Hz}$, is replicated using the Buttkicker with a frequency of $35 \mathrm{~Hz}$.

In conclusion, the Buttkicker system does not exactly replicate the vibrations in the aircraft; however, it is relatively close. As such, further investigation using qualitative tests helped by asking subjects questions about their perception of the vibrations in the simulator and also its impact on the simulator fidelity.

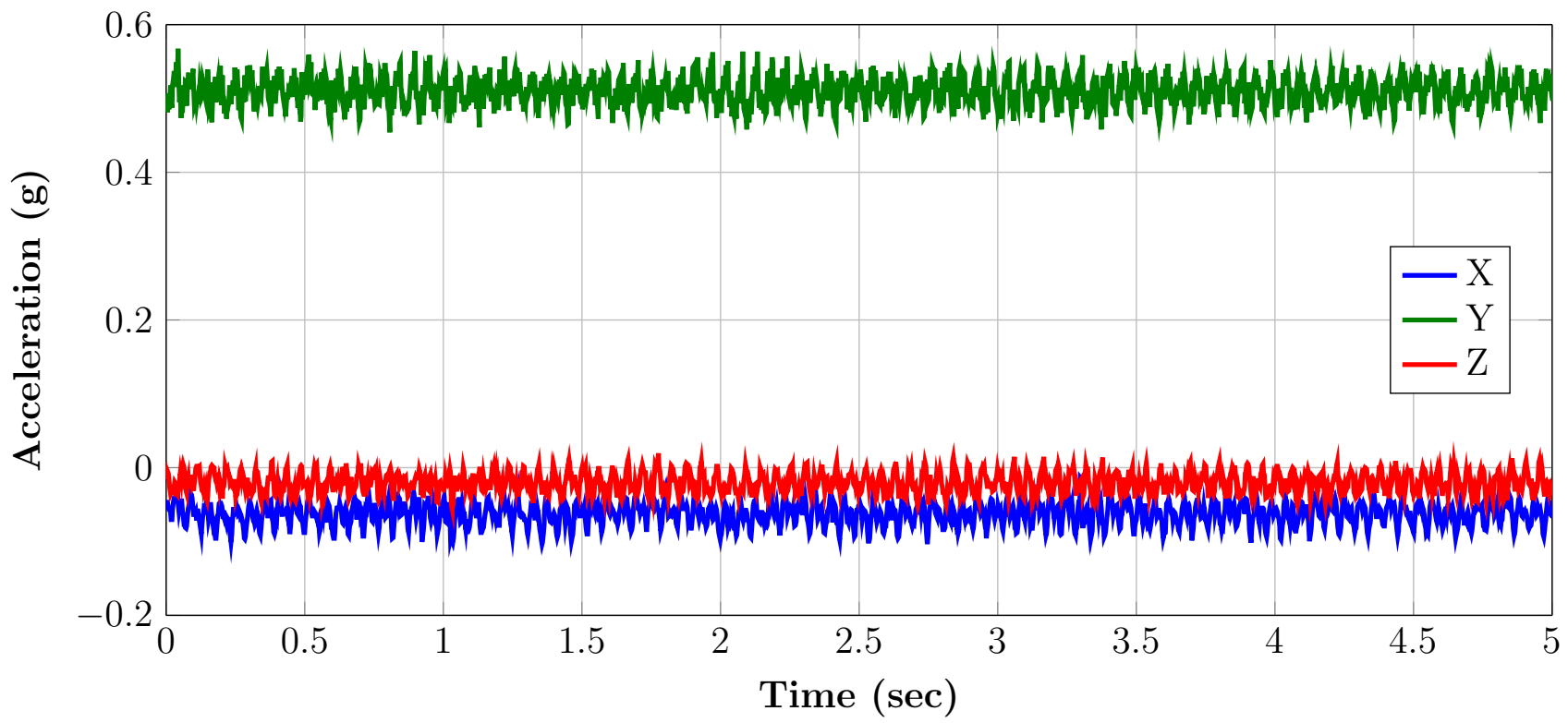

Figure 4.13: Acceleration versus time of the aircraft seat vibration in all directions while the engine was idling at 750 RPM. 


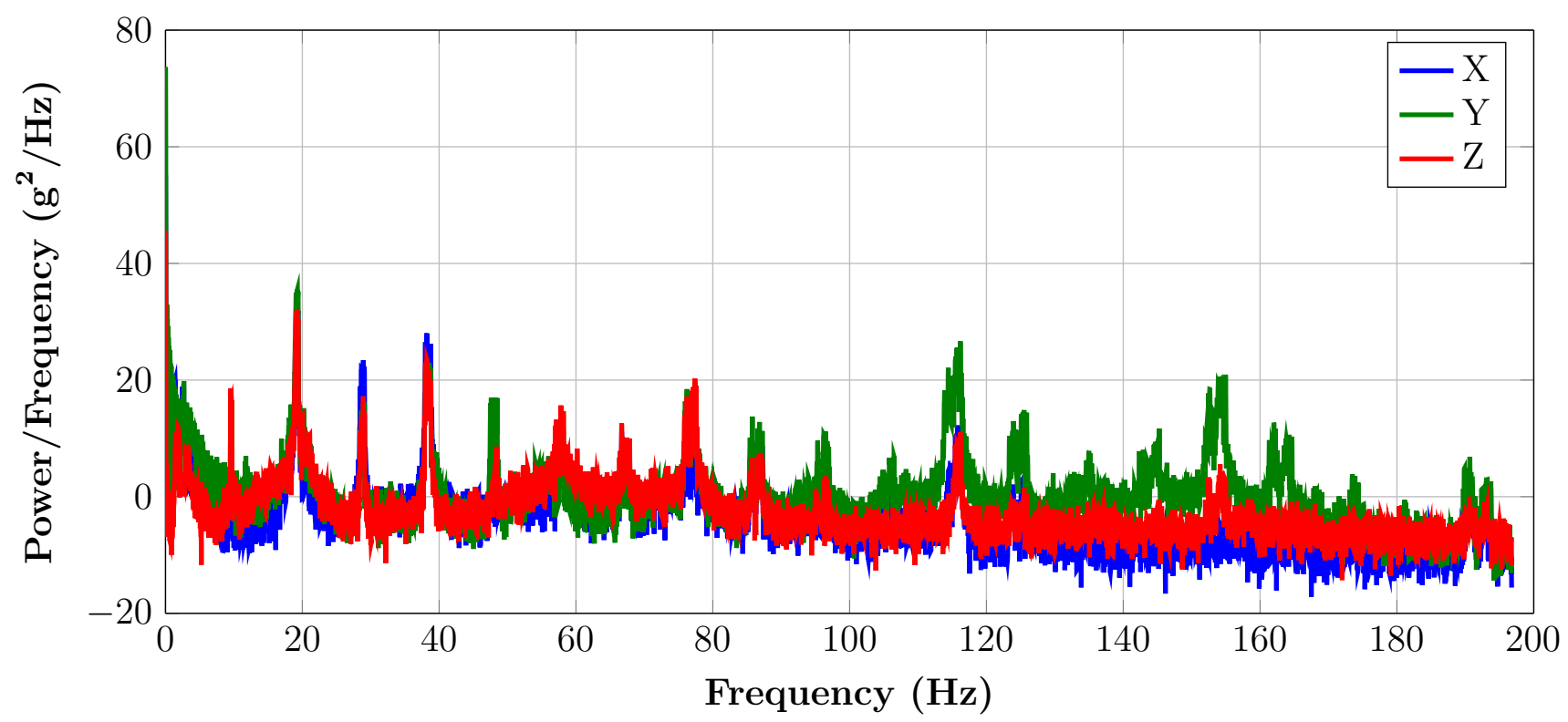

Figure 4.14: PSD analysis of the aircraft seat vibration in all three coordinate directions while the engine was idling at 750 RPM.

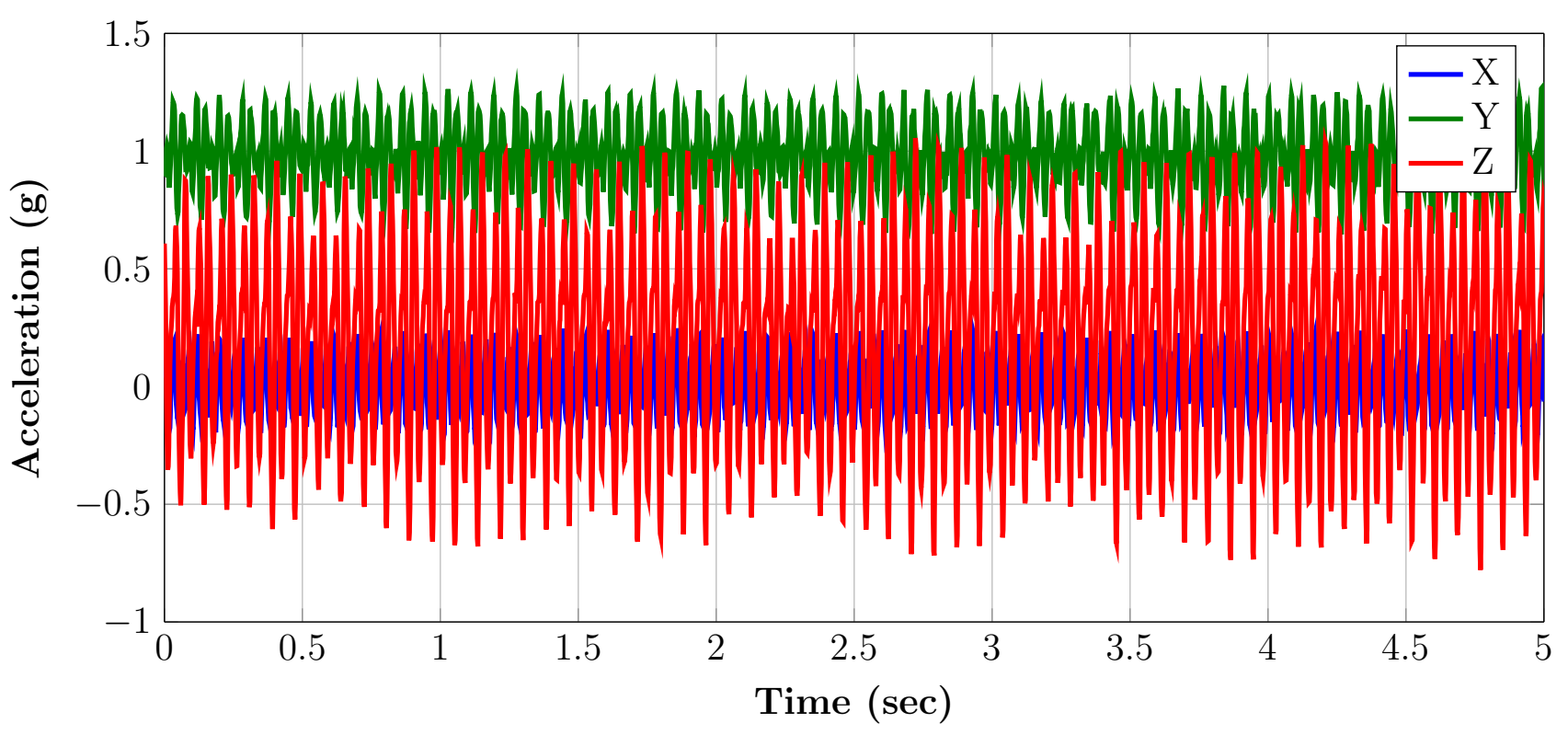

Figure 4.15: Acceleration versus time of the simulator seat vibration in all directions while the engine was idling at 750 RPM. 


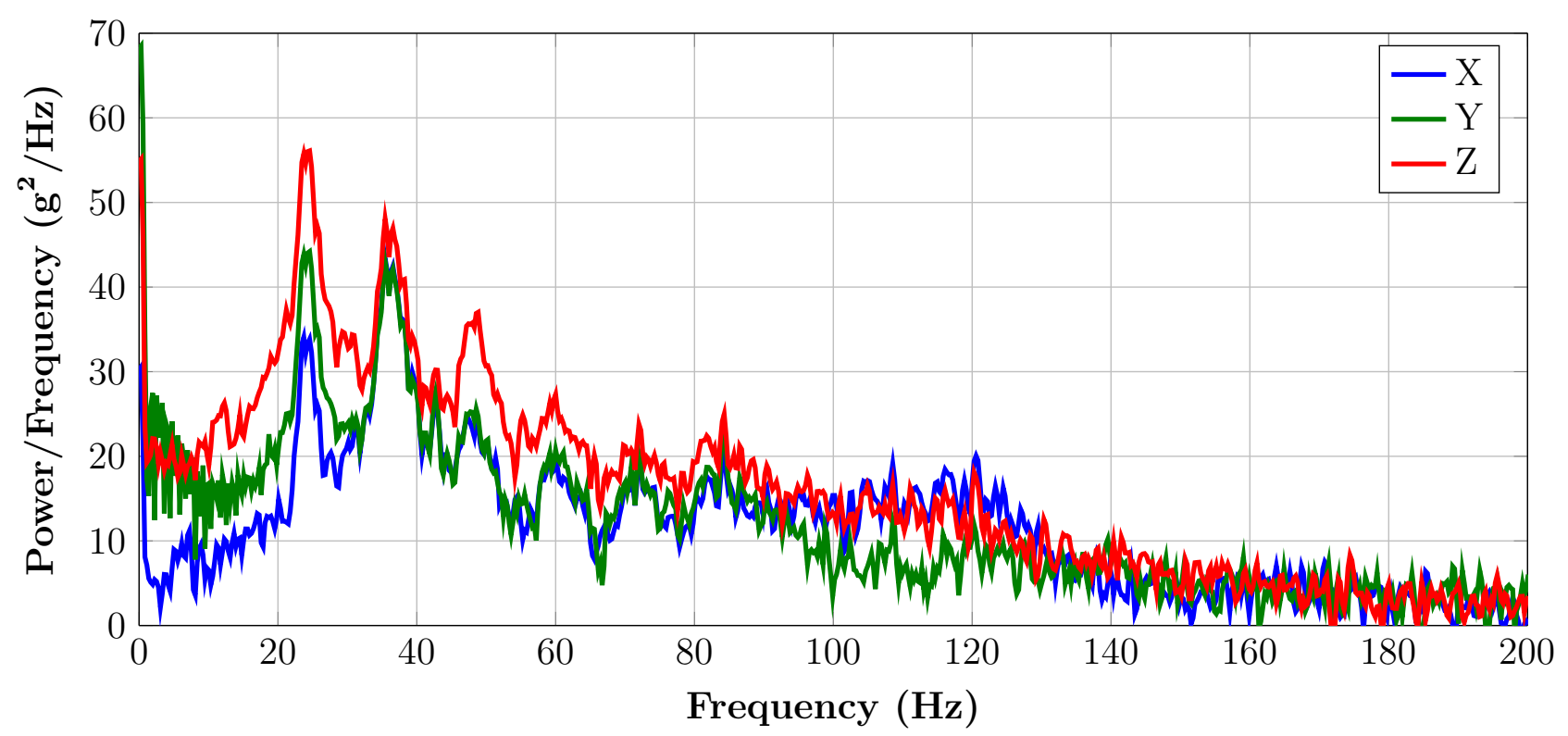

Figure 4.16: PSD analysis of the simulator seat vibration in all three coordinate directions while engine was idle running at 750 RPM.

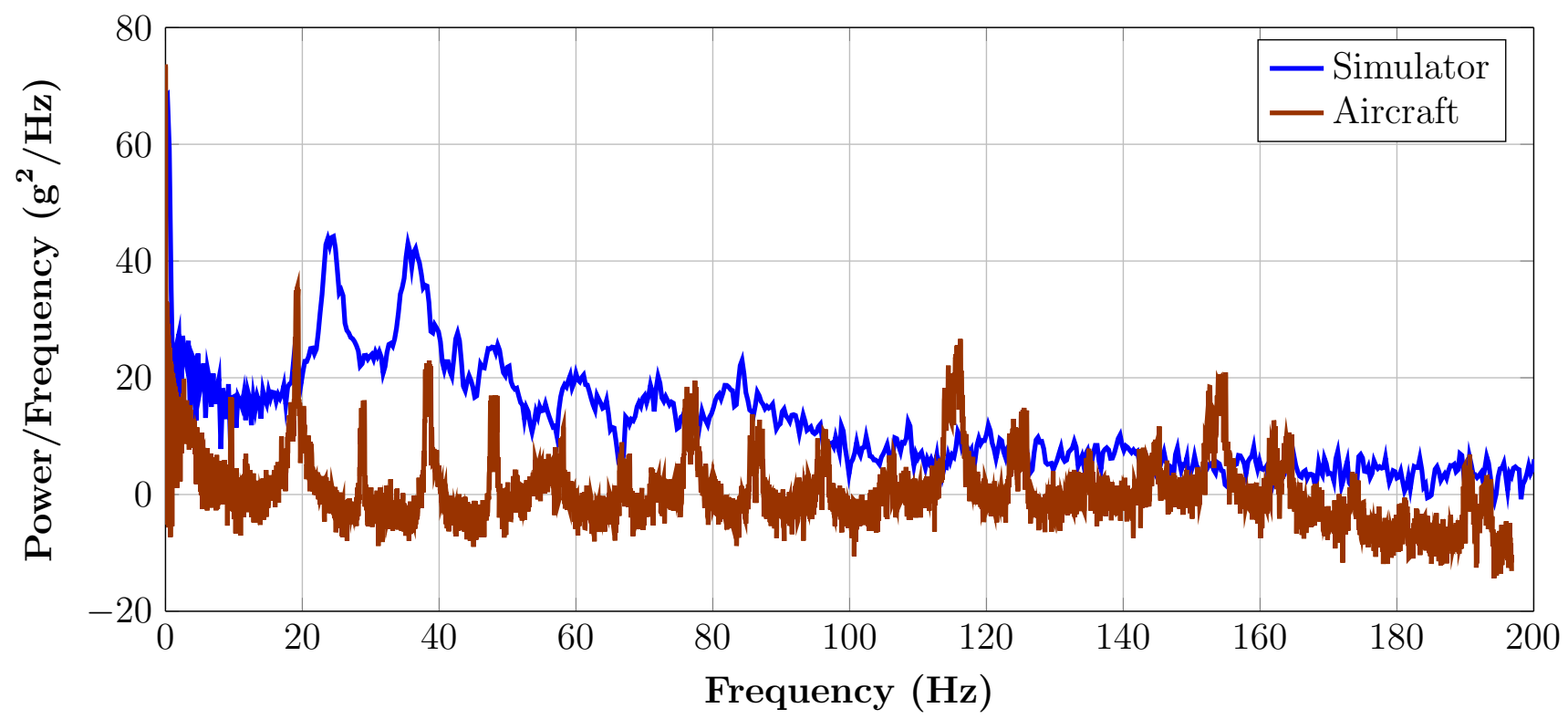

Figure 4.17: PSD comparison of the simulator and aircraft seat vibrations in the Y direction at $750 \mathrm{RPM}$. 


\subsubsection{Sound Data and Analysis}

The sound files are in WAV format and they were collected using a voice recorder throughout the flight tests, as is described in Chapter 4, and as well as in the simulator as shown in Figure 4.18. WAV file format is a normalized data set of the voltage signals captured by the recorder device. The amplitude of the voltages are normalized to the range between -1 and 1 . It is not easy to convert the normalized data into sound pressure as the WAV files do not contain the absolute magnitudes of sound pressure.

Alternatively, one can calculate the exact magnitudes by recording the SPL constantly throughout the flight. This was not done as the SPL meter used was not a high-frequency SPL recorder and it could only display the SPL at a desired moment (such as the maximum SPL). In the end, without having the exact magnitudes of the sound pressures, it was still possible to perform the PSD analysis and compare the simulator sound system with the actual aircraft, as the PSD demonstrates only the oscillations.

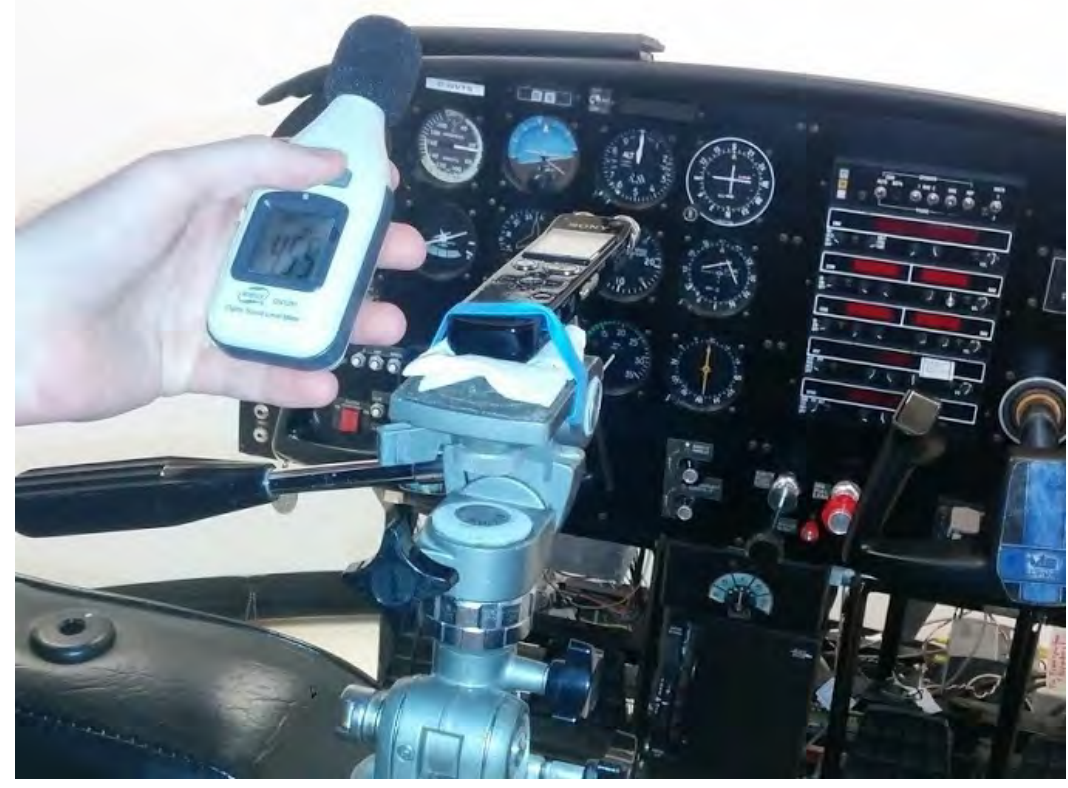

Figure 4.18: Simulator sound recording process. 
The same analysis, that was performed for vibration, was carried out on the sound files using the Welch method to analyze and compare the actual aircraft sound frequency components with the simulator ones. Like vibration, there were three different manoeuvres (taxi, cruise, engine idle) that were performed both in aircraft and the simulator where the corresponding engine noise were recorded using the sound recorder.

It is important to remember that engine sound in the simulator is only one sound file (at $1700 \mathrm{RPM}$ ) that is being changed constantly as response to varying engine RPM. This change is performed by X-Plane in real-time before sending the audio signals to the speakers.

Basically, the PSD comparison of the sound is to show that how accurate X-Plane can manipulate the single RPM sound file relative to the real aircraft. There is no specific reference to measure how close the simulator response to reality should be so that the pilot perceives the similar sound experience.

Figure 4.19 shows three seconds of the sound data points in the time domain. As is shown in Figure 4.24, the prevailing frequency components, both for the aircraft and the simulator, are below $1000 \mathrm{~Hz}$ and the most powerful ones fall below $200 \mathrm{~Hz}$. Also, as discussed in the previous section, the sound file that was implemented in X-Plane was recorded at 1700 RPM. Therefore, X-Plane has manipulated the sound file to pitch down the sound to a 750 RPM engine sound and this example is a good one for comparison. Also, PSD plots for other manoeuvres (cruise and taxiing) are illustrated in Appendix F.

As can be seen in Figure 4.20, the peak frequencies in the actual aircraft at 750 RPM happen about every $30 \mathrm{~Hz}$. In the simulator, however, the frequencies fluctuate more as is evident in Figure 4.22 and the comparison shown in Figure 4.23. Although the PSD analysis for the simulator in other manoeuvres, such as cruising (demonstrated in Appendix F), are closer to the aircraft, the frequency difference 
between the simulator and the aircraft in general suggests that the simulator may not be capable of generating the sound very precisely. However, the change in the pitch of the sound as a response to the engine RPM might be heard realistically while flying in the simulator. As such, like the vibrations, a qualitative experiment can provide a better way to test the modified sound files.

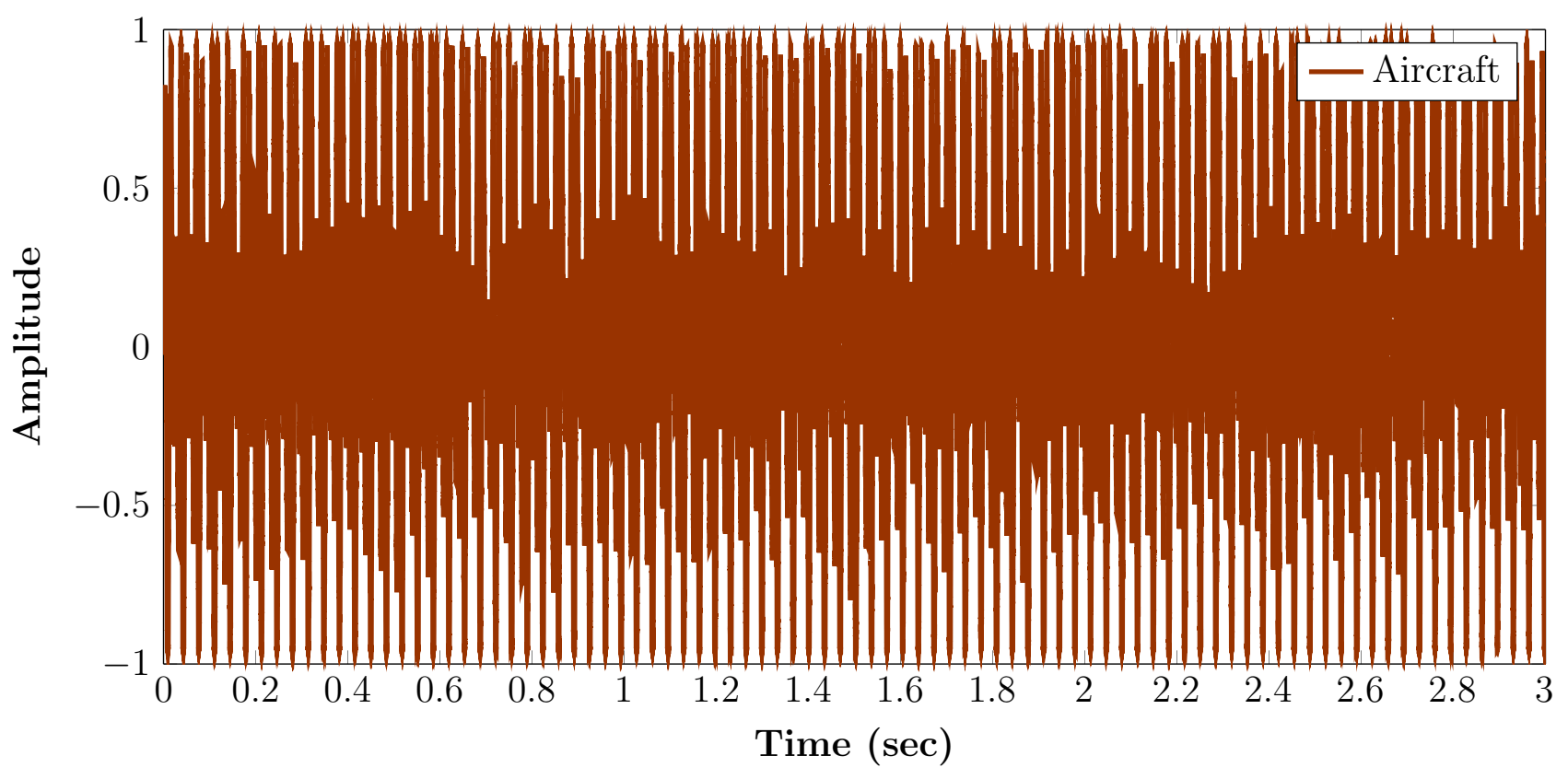

Figure 4.19: Normalized sound data in the time domain for the aircraft at 750 RPM. 


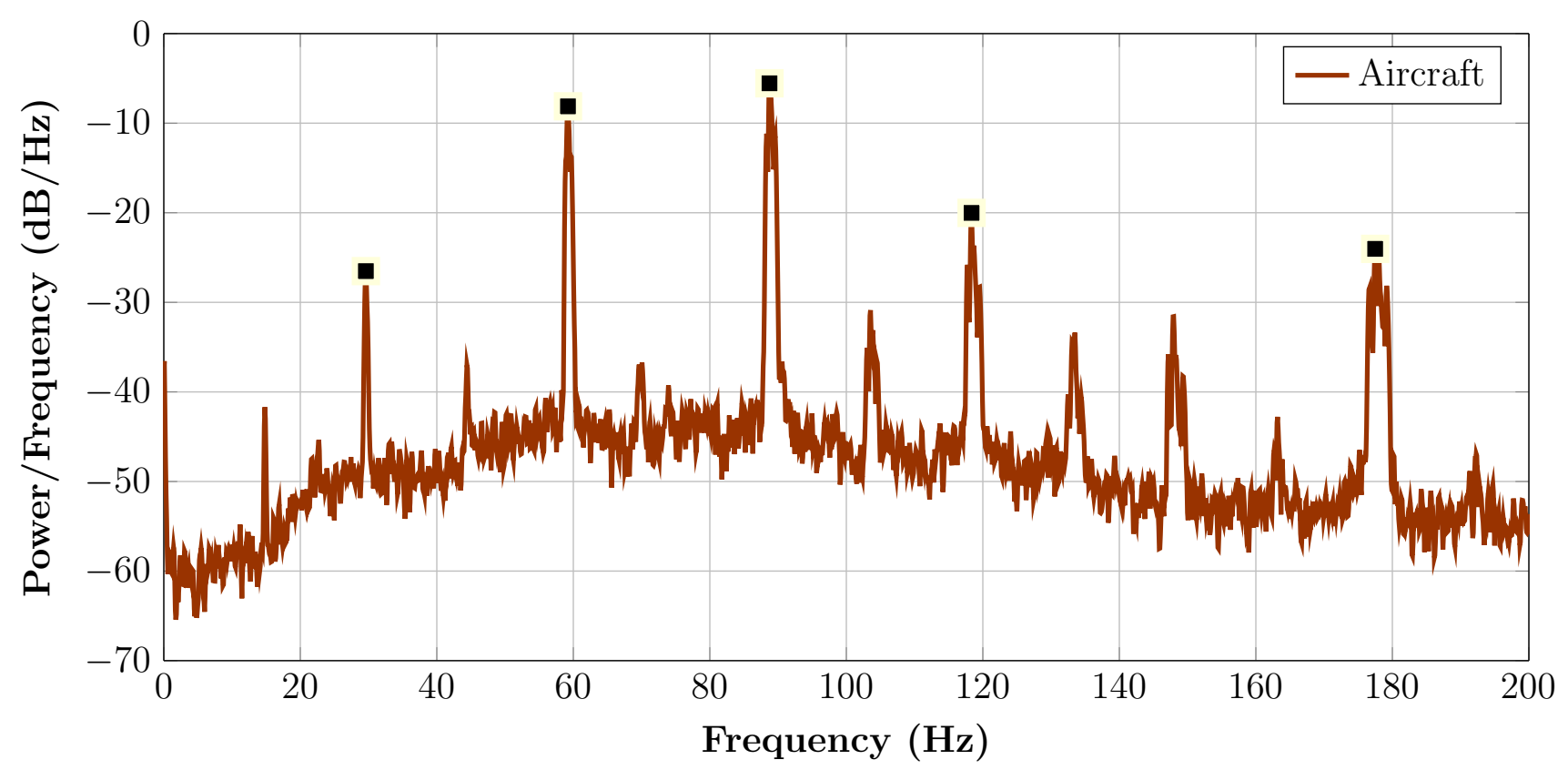

Figure 4.20: PSD analysis of the aircraft sound in the low frequency range at 750 RPM.

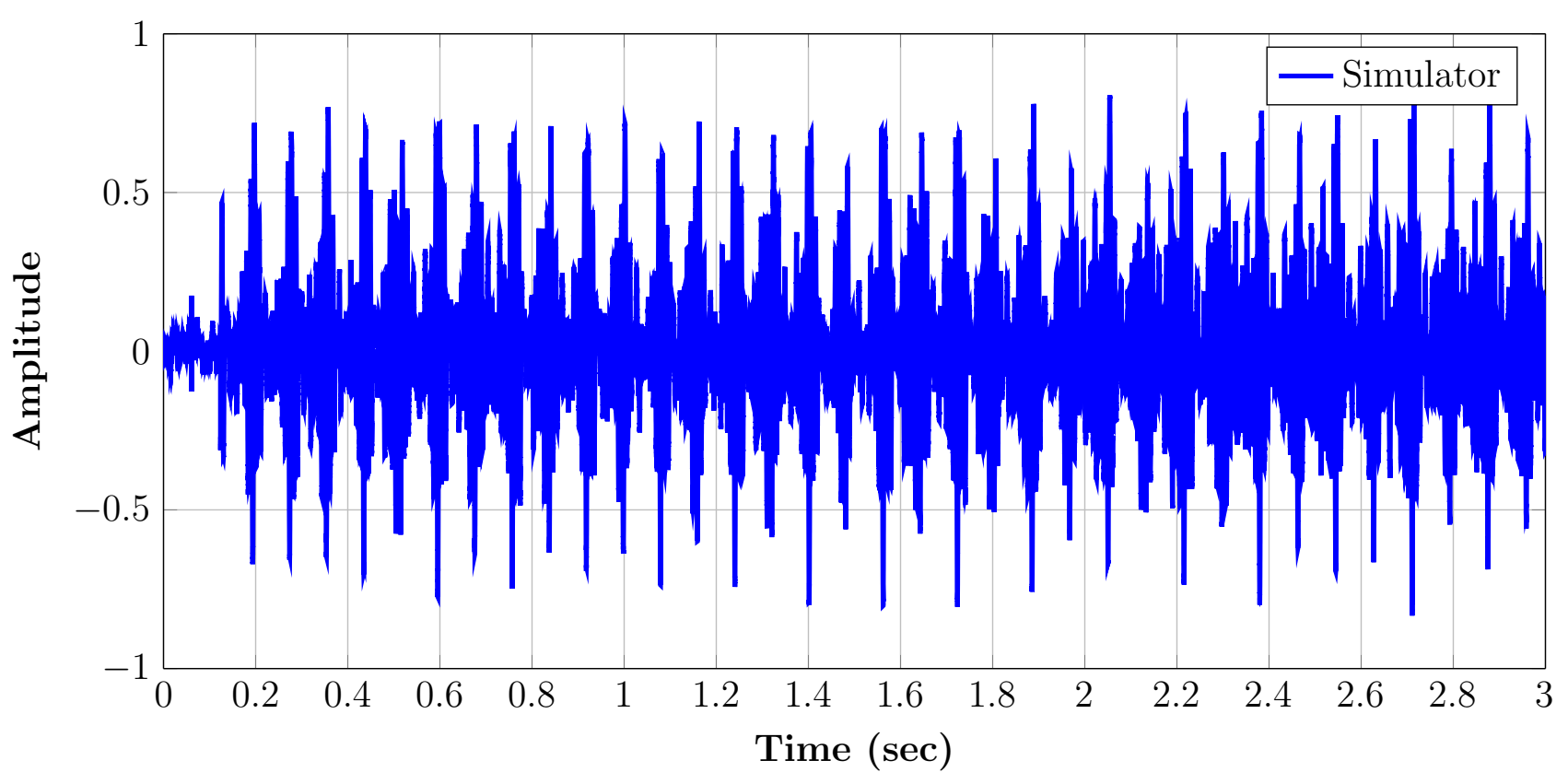

Figure 4.21: Normalized sound data in the time domain for the simulator at 750 RPM. 


\subsection{Qualitative Evaluation}

It was decided to perform a qualitative test of the simulator to assess its adequacy for the intended purpose by asking real pilots to describe their experiences in the

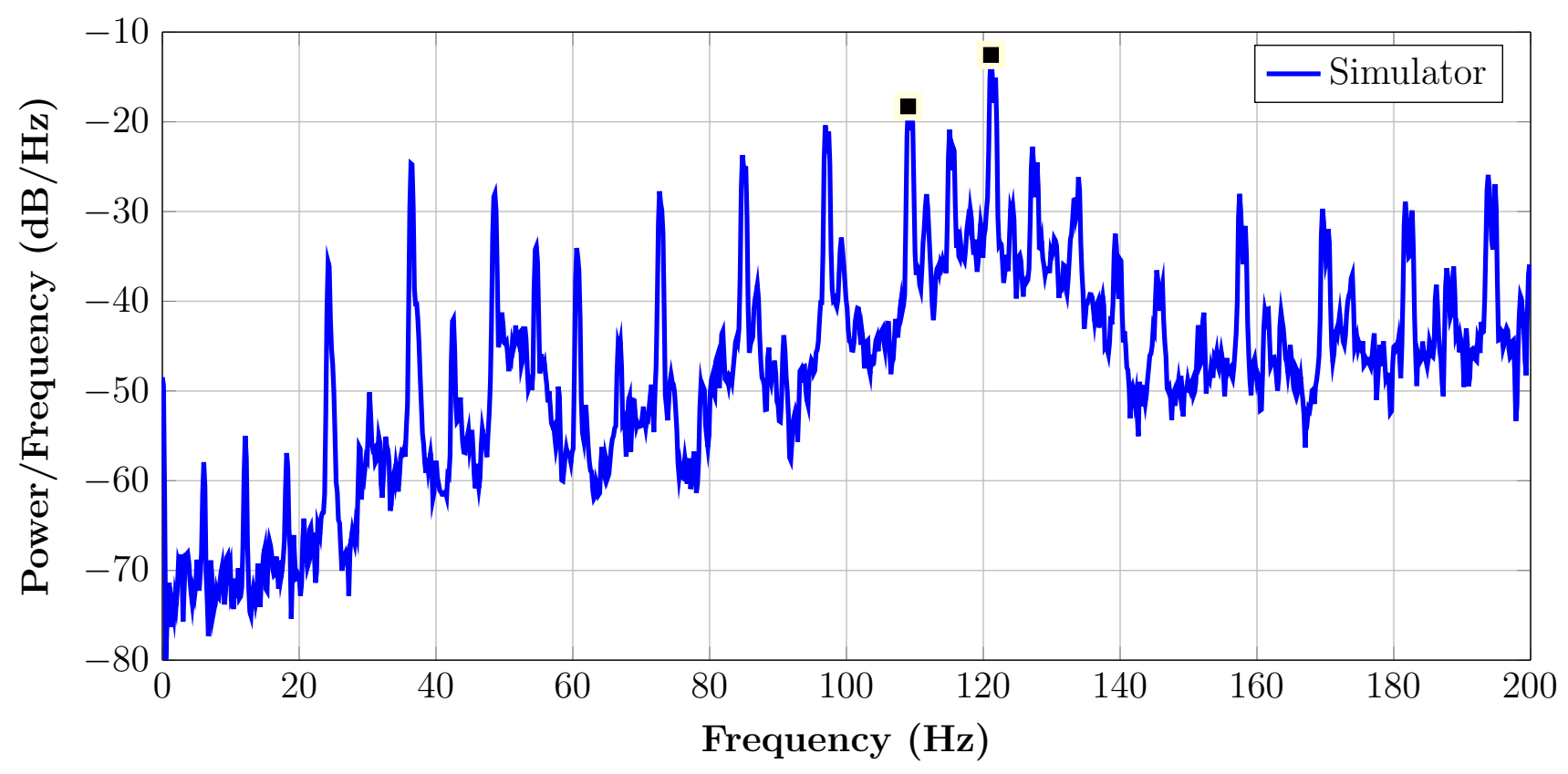

Figure 4.22: PSD analysis of the simulator in the low frequency range at 750 RPM.

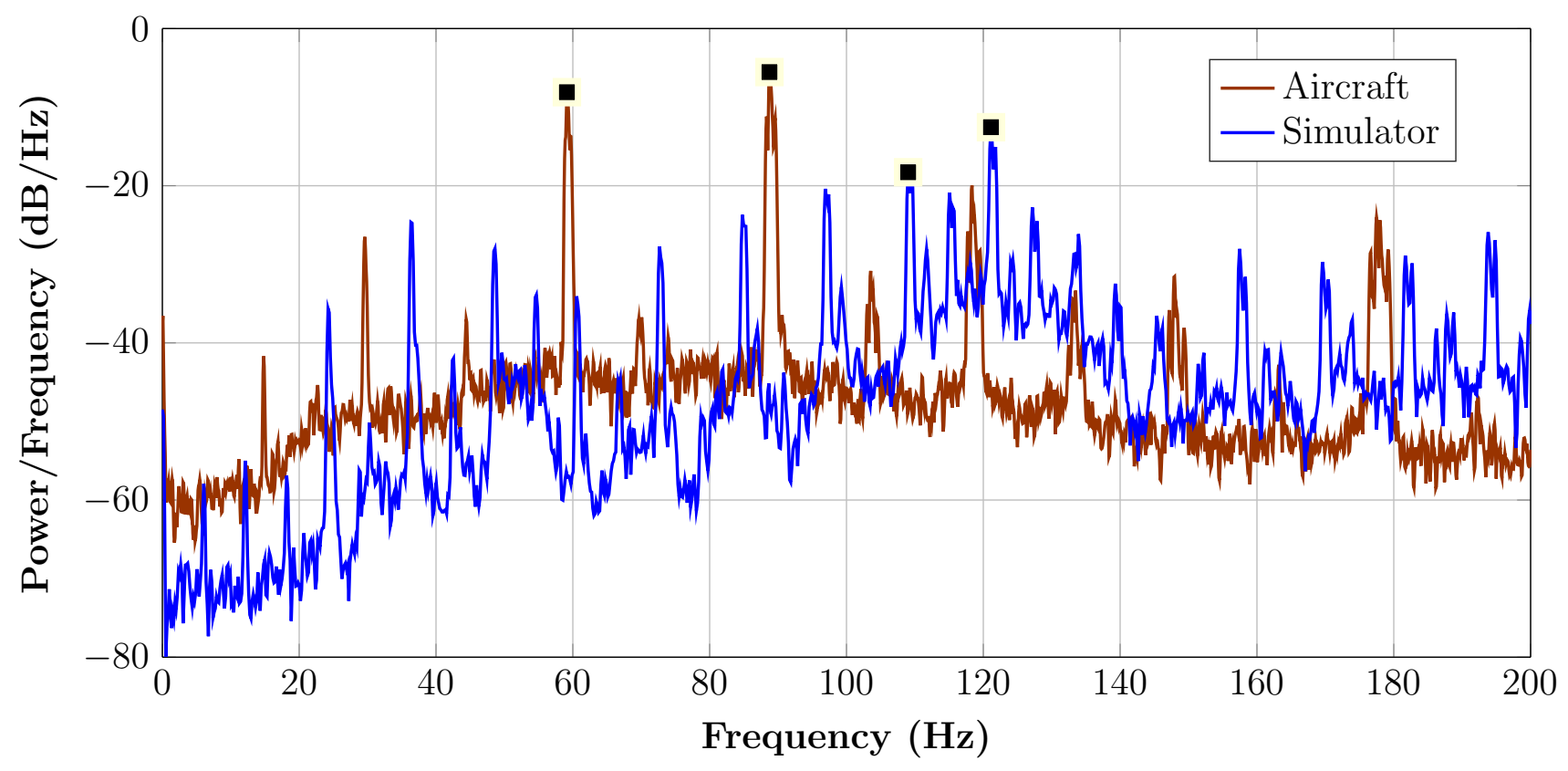

Figure 4.23: PSD analysis of the simulator and the aircraft sound at 750 RPM. 


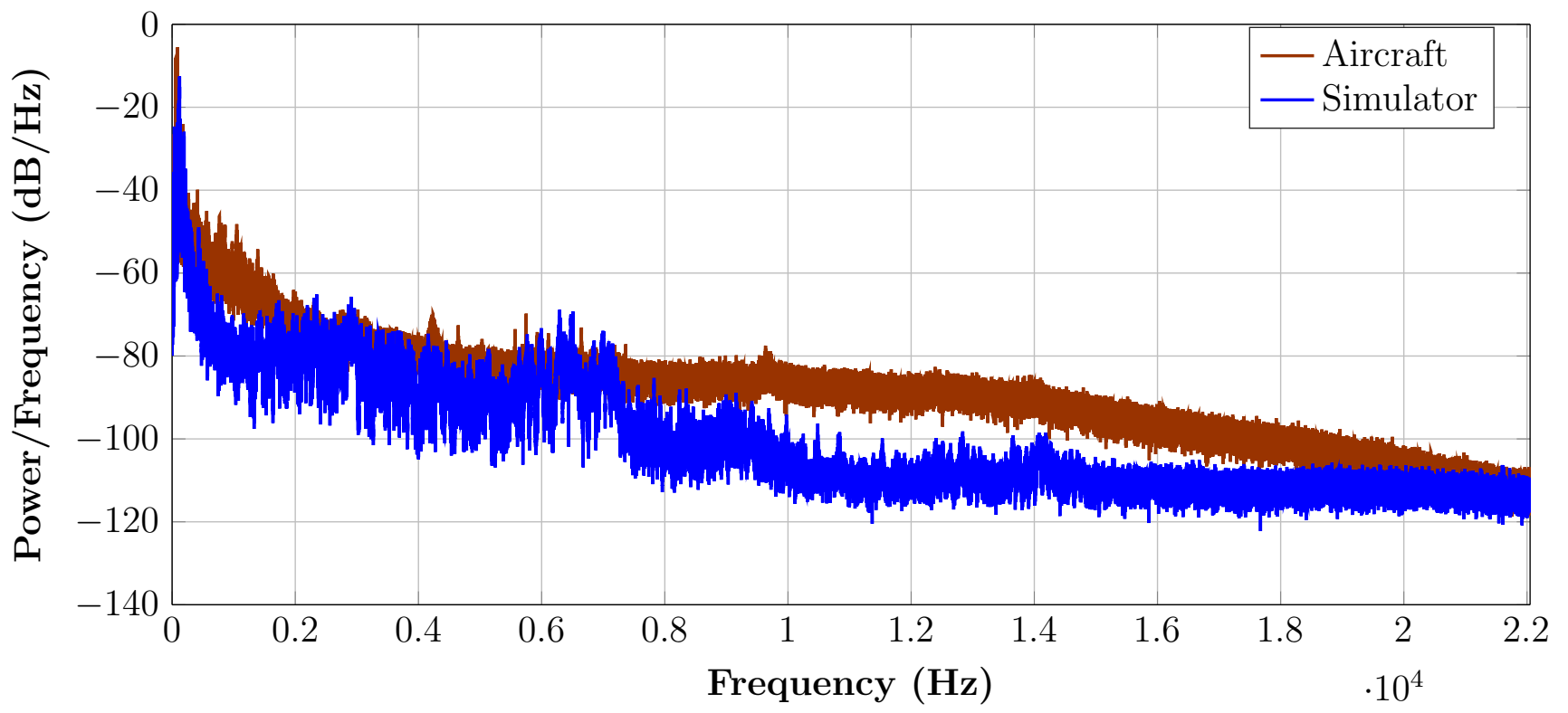

Figure 4.24: Full range PSD analysis of the simulator versus the actual aircraft sound.

simulator. At the time, the simulator was in a state where the visual system, sound system, and seat shakers were working properly, and thus it was found to be partly testable. Since the basic controls (pitch, yaw, roll, throttle, and trim wheel including control forces) were functional, thanks to the work of the other team members on the project, the CURVS could be flyable. Though, the instruments were not functioning at this stage of the project and they were not yet hooked up to the simulator. As such, it was decided to perform two separate tests. One would be a VFR version to test the functionality of the visual cueing, auditory cueing, and vibratory cueing systems by performing circuits to verify that the pilots get the pertinent feeling of flying. And secondly, an IFR test to examine all the instruments when ready. The main goals of the tests were to find the necessary fine-tuning procedures and incorporate required changes in advance of the final assembly of the simulator at the WWFC.

The VFR test was carried out by inviting eight pilots who have a PPL or were filling hours to get their licence. Since all the invited pilots had flown at CYOW 


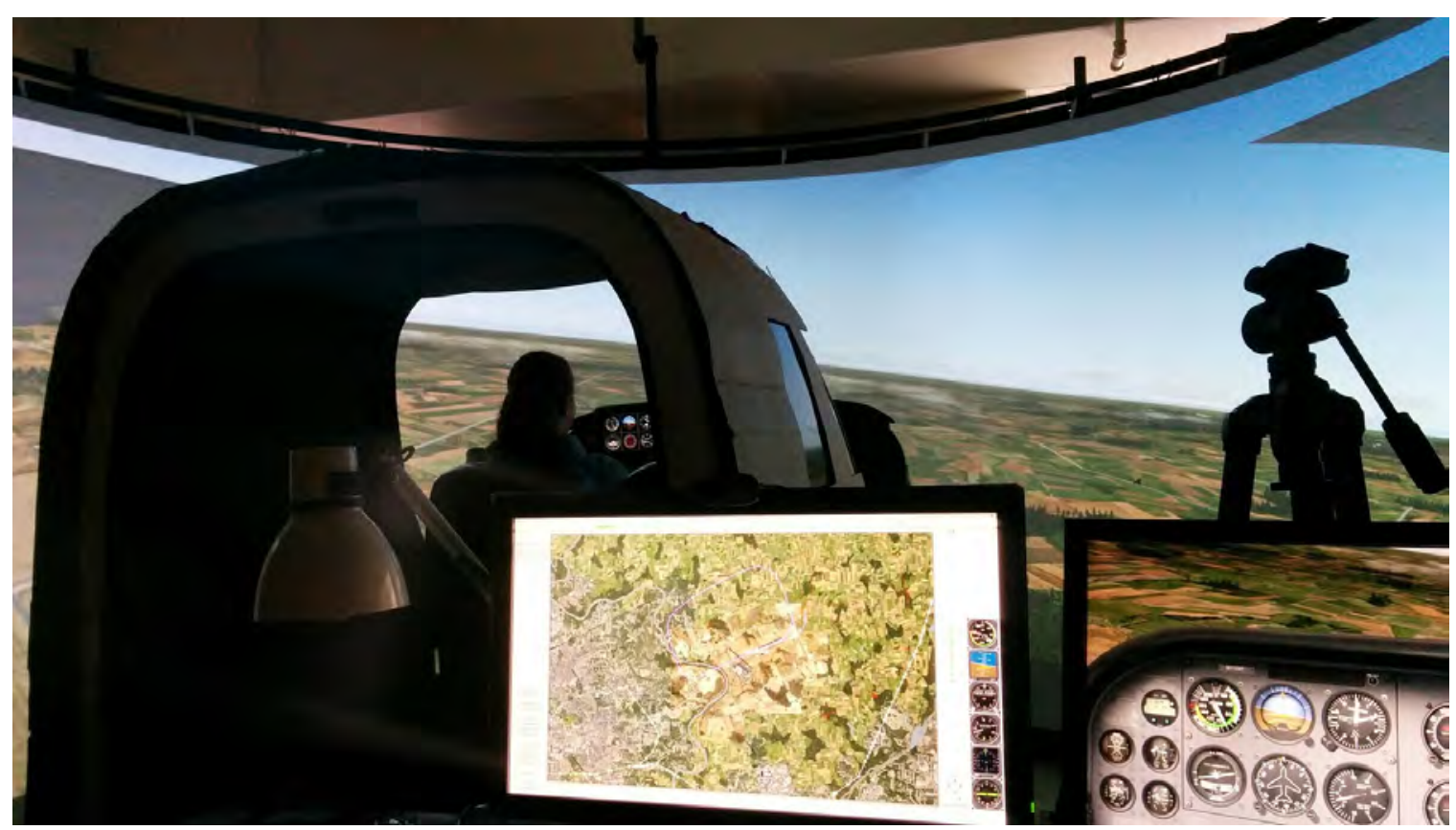

Figure 4.25: One of the participants flying circuits at CYKF.

many times before, they were asked to perform two circuits in CYOW (to get used to the simulator and not get distracted by the new airport environment at CYKF) and then two other circuits at CYKF as shown in Figure 4.25.

The scenery of the CYRO was downloaded from X-Plane's forum and was further modified by adding satellite images. Moreover, as the six pack instruments were not working at the time, an Android application was downloaded and used on an Android tablet. The app simulates the basic six pack instruments and communicate with the simulator as the tablet is connected to the same network as the CURVS computers through a UDP data transfer connection. The tablet was attached to the instrument panel so that the pilots had sufficient instruments to fly circuits. Figure 4.26 shows the six pack instruments placed on the simulator dashboard within view of the pilot.

After the test, participants were asked to complete a questionnaire in which 27 


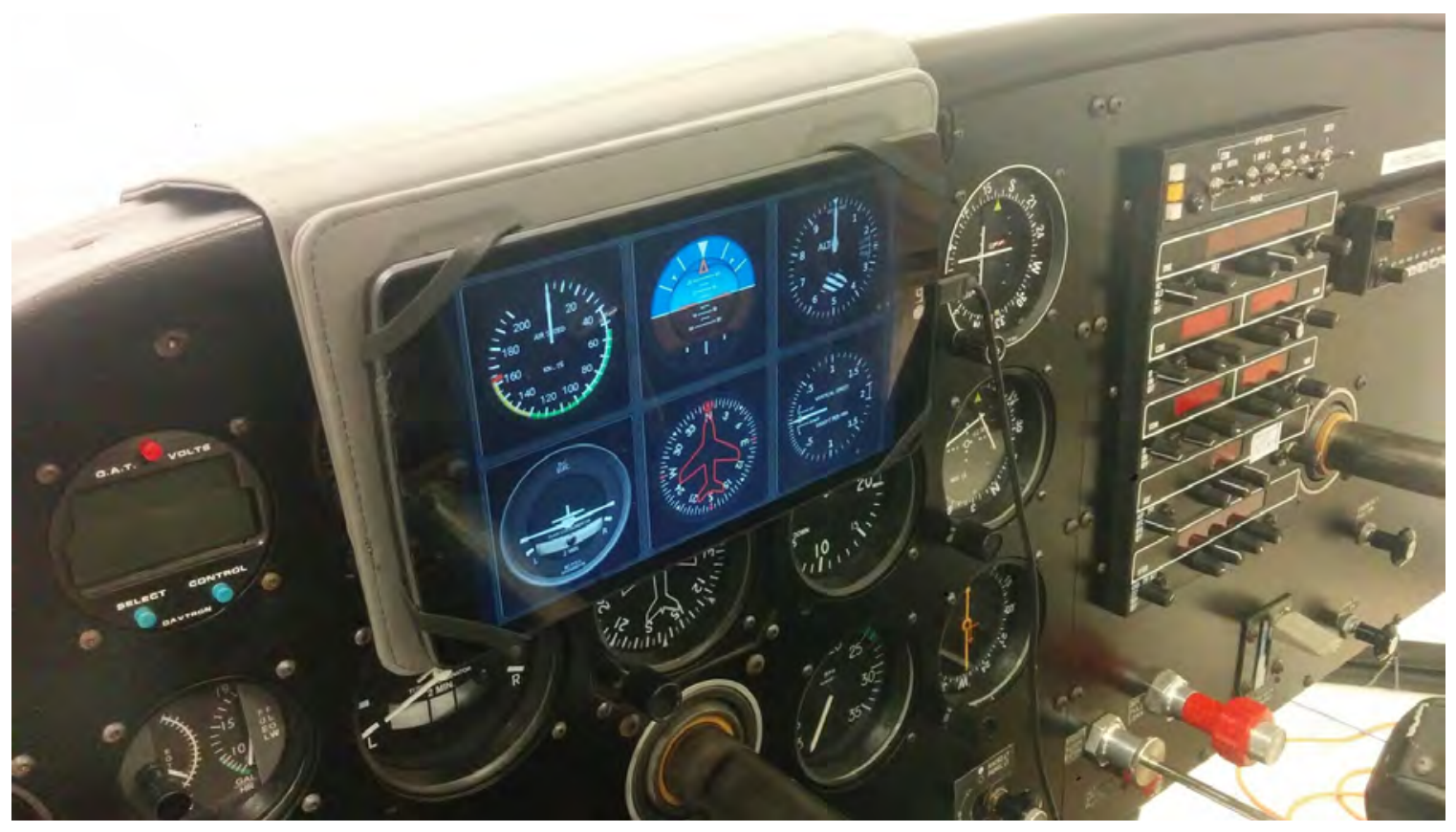

Figure 4.26: The tablet implemented to replicate the six pack instruments.

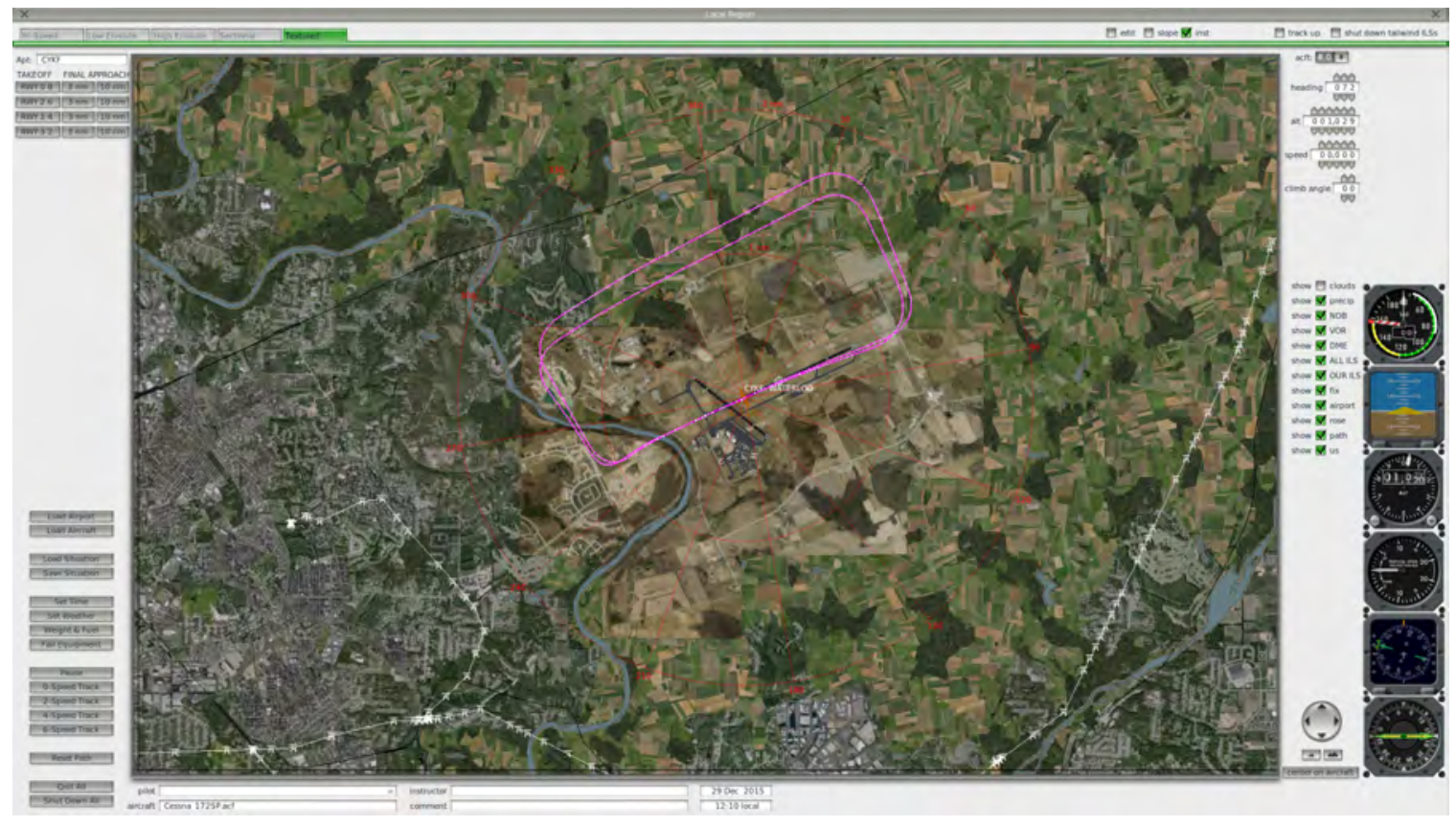

Figure 4.27: The circuit pattern at CYKF flown by one of the participants. 
questions were posed about the influence of visual cueing, auditory cueing, and vibratory cueing in their simulator flying experience in the CURVS. The questionnaire was adopted from a similar simulator test conducted by NASA [52]. It consisted of multiple Yes/No and ranking questions for each cueing system and participants were asked to comment on each question. The questions target FOV, framerates, screen resolution, the fidelity of the engine noise, and the effects of the seat vibration on the simulation experience. The questions are numbered from 1 to 27 making it simpler to summarize results in a table. A copy of the questionnaire can be found in Appendix $\mathrm{H}$ and the results of the tests are depicted in Tables 4.4 and 4.5.

Almost all the participants enjoyed the CURVS experience and found it a very promising project overall. The participants varied in age, gender, flying hours, and pilot licence degrees. They ranked the overall visual system functionality good or excellent (according to the ranking system) in the aspects of FOV, screen resolution, and framerate. Particularly, almost all the pilots commented on the extra HFOV for OSV and found it very realistic and beneficial, making the circuit practices easier for ab-inito students. With regards to the FOV, one of the participants commented that "from the pilot side you see basically everything you need to".

The results depict that the pilots had no trouble with the screen resolution, and they all claimed they could recognize all the scenery contents clearly. It can be interpreted that the distances of the screen from the PEP and the projectors are effectively sufficient.

Although during the tests, especially while flying over Rockcliffe scenery, there was some jitteriness and lags in the simulation (almost no lag with the customdeveloped Waterloo scenery though), all the pilots asserted that the simulation was fairly smooth (mostly ranked EXCELLENT except for two GOOD choices). This has to be proven using an specific test guides described in the simulator manual [2] and [31] for certification. 
The participants found the audio cueing fidelity high and close to reality. They mentioned that the actual C172's engine noise is much louder than what they experienced in the CURVS; however, they didn't expect more volume than what they were hearing throughout the experiments and mentioned that it is sufficient for a simulator. Two of the pilots commented that the electric flaps sound needs to be tuned and amplified. Therefore, after the tests the flap sound was tuned and amplified using the Audacity software. Despite pilots describing a fairly realistic relationship between the throttle inputs and the engine sound at different RPMs, they could not clearly recognize whether or not the change in the pitch of the sound is realistic. One of the pilots mentioned that it needs further studies with the help of professional C172 pilots for tuning.

Participants believed that the seat vibrations played a positive role in the fidelity of the simulation. Qualitatively, $62.5 \%$ of them contend that they felt the vibrations were effective during the takeoff and touchdown transitions (air-to-ground and ground-to-air). The pilots found the vibrations more prominent while on the ground (during takeoff, on runway, taxing, etc.) and they claimed the feeling is quite realistic so there is no need for further tuning. A summary of the important rating questions is illustrated in the Figure 4.28.

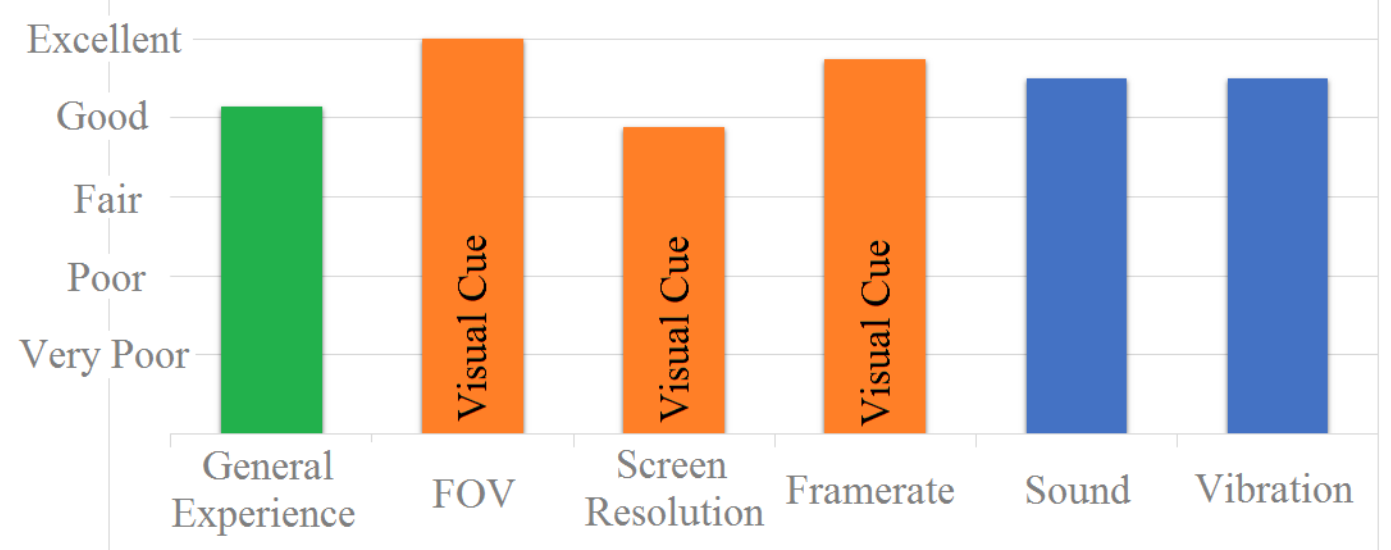

Figure 4.28: The results of the rating questions. 
Table 4.4: Questionnaire results - Participants 1 to 4.

\begin{tabular}{|c|c|c|c|c|}
\hline Question & Participant 1 & Participant 2 & Participant 3 & Participant 4 \\
\hline 1 & Good & Excellent & Excellent & Good \\
\hline 2 & Good & Excellent & $\mathrm{N} / \mathrm{A}$ & Good \\
\hline 3 & Excellent & Excellent & Excellent & Excellent \\
\hline 4 & Yes & Yes & Yes & Yes \\
\hline 5 & Yes & Yes & Yes & Yes \\
\hline 6 & Yes & Yes & Yes & Yes \\
\hline 7 & Yes & Yes & Yes & Yes \\
\hline 8 & Fair & Good & Good & Good \\
\hline 9 & No & No & No & No \\
\hline 10 & Yes & Yes & Yes & Yes \\
\hline 11 & Yes & Yes & Yes & Yes \\
\hline 12 & Yes & Yes & Yes & Yes \\
\hline 13 & Good & Good & Excellent & Excellent \\
\hline 14 & Yes & Yes & Yes & Yes \\
\hline 15 & Yes & Yes & No & Yes \\
\hline 16 & No & Yes & No & No \\
\hline 17 & No & Yes & No & No \\
\hline 18 & Good & Excellent & Good & Excellent \\
\hline 19 & Yes & Yes & $\mathrm{N} / \mathrm{A}$ & Yes \\
\hline 20 & Yes & Yes & Yes & Yes \\
\hline 21 & Yes & No & $\mathrm{N} / \mathrm{A}$ & Yes \\
\hline 22 & No & No & Yes & No \\
\hline 23 & Good & Excellent & Good & Excellent \\
\hline 24 & 3 & 4 & 3 & 4 \\
\hline 25 & Takeoff & Takeoff & Start up & On Ground \\
\hline 26 & Yes & Yes & No & No \\
\hline 27 & Yes & Yes & Yes & Yes \\
\hline
\end{tabular}


Table 4.5: Questionnaire results - Participants 5 to 8.

\begin{tabular}{|c|c|c|c|c|}
\hline Question & Participant 5 & Participant 6 & Participant 7 & Participant 8 \\
\hline 1 & Poor & Good & Fair & Fair \\
\hline 2 & Excellent & Excellent & Poor & Good \\
\hline 3 & Excellent & Excellent & Excellent & Excellent \\
\hline 4 & Yes & No & Yes & Yes \\
\hline 5 & Yes & Yes & Yes & Yes \\
\hline 6 & Yes & Yes & Yes & Yes \\
\hline 7 & Yes & No & $\mathrm{N} / \mathrm{A}$ & $\mathrm{N} / \mathrm{A}$ \\
\hline 8 & Excellent & Good & Fair & Good \\
\hline 9 & No & Yes & Yes & No \\
\hline 10 & Yes & Yes & Yes & Yes \\
\hline 11 & Yes & Yes & Yes & Yes \\
\hline 12 & Yes & Yes & Yes & $\mathrm{N} / \mathrm{A}$ \\
\hline 13 & Excellent & Excellent & Excellent & Excellent \\
\hline 14 & Yes & Yes & No & $\mathrm{N} / \mathrm{A}$ \\
\hline 15 & Yes & Yes & Yes & No \\
\hline 16 & Yes & Yes & Yes & Yes \\
\hline 17 & No & No & No & No \\
\hline 18 & Excellent & Excellent & Good & Good \\
\hline 19 & Yes & Yes & Yes & Yes \\
\hline 20 & Yes & Yes & Yes & Yes \\
\hline 21 & Yes & Yes & Yes & $\mathrm{N} / \mathrm{A}$ \\
\hline 22 & Yes & No & No & No \\
\hline 23 & Excellent & Good & Excellent & Good \\
\hline 24 & 3 & 3 & 3 & 3 \\
\hline 25 & Taxing & Ground Roll & Takeoff & Runway \\
\hline 26 & No & Yes & Yes & Yes \\
\hline 27 & Yes & Yes & Yes & $\mathrm{N} / \mathrm{A}$ \\
\hline
\end{tabular}




\section{Chapter 5}

\section{Conclusion}

\subsection{Summary and Conclusion}

In this thesis visual, audio, and vibratory cueing systems for a cost-effective FSTD were designed, developed, and evaluated. It was found out that by using commercialoff-the-shelf components, building an inexpensive immersive wraparound visual system, sound system, and seat vibration system for high-fidelity flight simulators, especially for ab-initio training, is feasible. As recent studies, discussed in Chapter 1, show that motion platforms do not play a significant role in the transfer of training for ab-inito training, it was proven in this research that a high-quality visual system in conjunction with vibration can be a beneficial compromise.

Also, it was demonstrated that constructing a fast, high-quality, PC-based image generator (hardware and software) with complex scenery content that runs smoothly in real-time not only is achievable but also can surpass acceptable standards. A lot of freeware and affordable software is currently available with which one can design and build a high-quality 3D virtual environment. With regards to that, there is also a large connected network of hobbyists that are constantly contributing to the

advancement of visual content. As such, the cost of software development for flight simulators is significantly lower than it as been in the past. 
The CURVS project commenced with three preliminary flight tests to evaluate abinitio flight training requirements and also to collect relevant data. After the FSTD was built, post-development qualitative and quantitative assessments were performed. In the quantitative tests, acceleration and sound data, as well as brightness data were collected to perform analysis. For vibration and sound power spectral analysis was performed to evaluate differences between the simulator and the aircraft. Despite the fact that PSD analysis suggests that the simulator does not exactly replicate the aircraft responses, the findings of the qualitative tests proved that the implemented vibration and sound cueing play a substantial beneficial role in the simulation realism. Therefore, adding high-quality COTS components for sound and vibration especially as they do not cost much, is notably advantageous.

Finally, as the project still was not finished while this thesis was written, the total cost of the CURVS FSTD was unclear. However, the price of the implemented cueing systems (visual, vibration, and sound), including the image generator, was estimated about $14,000 \$$ in 2015. Assuming that all the other components of the CURVS can be built cost-effectively, The total of price of the FSTD is relatively lower than its competitors in the market (within the range of a few hundred thousand dollars). Moreover, it is important to note that CURVS was a research project meaning the corresponding budget will become lower when assembled in a production line.

\subsection{Contributions}

The CURVS project is an indication that high-quality cueing systems for type specific FSTDs, that take advantage of PC-based COTS components to reduce the corresponding costs is feasible. In this thesis, the design, development, and analysis of 
high-quality visual, audio, and vibratory (as a standalone) cueing systems are demonstrated. Also, for evaluation purposes, flight tests were performed in an actual C172 aircraft in which multiple sound and vibration data were collected for quantitative comparison.

The primary contribution of this thesis was the implementation of multifarious cueing systems to advance the previous methods proved in the ADL for building cost-effective FSTDs.

The feasibility of constructing an economical wraparound display with large enough FOV (220 degrees) for over-shoulder viewing during landing was assessed and proved to be possible and desirable. This was accomplished by taking advantage of COTS projectors and blending software, and as well as a custom-built screen frame and COTS screen fabric. Also, high quality audio and seat shaking systems were designed and developed. Throughout the research, it was found that a high-quality visual system combined with a vibratory cueing system reduces the need for motion platform which in turn significantly reduces the FSTD cost.

Finally, qualitative and quantitative assessment of the simulator were carried out and the results showed that there is a high potential in developing industrialized high-quality cueing systems for FSTDs that can be built based on COTS components.

\subsection{Future work}

According to the assessment of outcomes of the CURVS project, the following considerations are offered for the next phase of the project.

1. An improved visual system by taking into account:

- The feasibility of achieving a greater FOV without loosing resolution within 
the same projection architecture;

- The possibility of implementing a cost-effective collimated display (vacuum mirror system) to reduce the parallax effect;

- The practicality of employing one mega PC (with a high-end COTS hardware architecture) instead of four networked PCs with the same visual system. The potential advantage is that it can lower the cost of all the software licenses by three to four times as well as reduce the electricity consumption of the simulator;

- Possible use of higher-quality orthophotos for the whole area of interest of the flight school;

- The use of other simulator software in the market such as Prepare3D in comparison to X-Plane for professional simulator usage.

2. A better and higher quality sound system by:

- Recording more sound files from inside and outside of the aircraft;

- Implementation of better wind sound;

- The possibility of finding an optimization algorithm to shift the recorded sound pitch more realistically.

3. An optimization algorithm to produce sound files (only for vibration) that converge to the desired frequencies for the Buttkicker.

4. A comprehensive qualitative study and evaluation of the simulator while assembled and in use in the flight school.

The research must continue to advance the FSTDs and reduce the costs associated with flight simulation technologies so that even small flight schools can employ highquality simulator training services. 


\section{References}

[1] D. Alletron. Principles of Flight Simulation. American Institute of Aeronautics and Astronautics, Wiley, Reston, VA, USA, 2009.

[2] Aeroplane and Rotorcraft Simulator Manual: TP 9685E. Transport Canada, January 1998.

[3] TRU Simulation. http://www.trusimulation.com/. Accessed: 2015-06-16.

[4] T. Brady N. D. Macchiarella and P. K. Arban. High Fidelity Flight Training Devices in the Training of Ab Initio Flight Students. 24th Digital Avionics Systems Conference, 2005-01.

[5] Waterloo Wellington Flight Centre. Personal communication with CEO, Bob Connors in June 2015.

[6] Ottawa Aviation Services Simulator. Personal communication with CEO, Cedric Paillard in June 2015.

[7] D. Winter. The First of the Few: Fighter Pilots of the First World War. University of Georgia Press, Georgia, USA, 1982.

[8] J. M. Rolfe and K. J. Staple. Flight Simulation. Cambridge University Press, Cambridge, England, 1986.

[9] R. E. Barrette. Flight Simulator Visual Systems: an Overview. SAE Aerospace Technology Conference, Long Beach, CA, USA, 1986.

[10] AVSIM. https://www.avsim.com. Accessed: 2015-10-29.

[11] Precision Manuals Development Group. https://www.precisionmanuals .com. Accessed: 2015-10-29. 
[12] Suzanne Swaine. Development of a Cost-effective High-fidelity Type-specific Flight Simulator with Emphasis on Flight Modelling. Carleton University, Carleton University, Ottawa, ON, Canada, April 3, 2014.

[13] Jonathan Plumpton. Development of a Full-fight Simulator for Ab-initio Flight Training with Emphasis on Hardware and Motion Integration. Carleton University, Carleton University, Ottawa, ON, Canada, April 3, 2014.

[14] ALSIM FTD. http://www.alsim.com/flight-training-solutions.php. Accessed: 2015-08-16.

[15] Mechtronix FTD. http://www.mechtronix.com/fft-series/. Accessed: 2015$08-16$.

[16] Wikipedia - Link Trainer. https://en.wikipedia.org/w/index.php?title= Link_Trainer\&oldid=674236399. Accessed: 2015-08-16.

[17] Pilot Examiner Manual: TP 14277E. Transport Canada, January 2013.

[18] Federal Aviation Administration. Far/Aim 2013: Federal Aviation Regulations/Aeronautical Information Manual. US department of transportation, March 2013.

[19] My Cockpit. http://www.mycockpit.org/forums/forum.php. Accessed: 201507-16.

[20] Cockpit Builders. http://www. cockpitbuilders.com/community/index.php. Accessed: 2015-07-16.

[21] Personal communication with Chris Hobbs, instructor pilot and author of the book (Flying Beyond) in June 2015.

[22] X-Plane website. http://wiki.x-plane.com/Setting_Up_X-Plane_for_ Best_Performance. Accessed: 2015-06-13.

[23] N. Frstenau S. R. Ellis and M. Mittendorf. Frame Rate Effects on Visual Discrimination of Landing Aircraft Deceleration: Implications for Virtual Tower Design and Speed Perception. PROCEEDINGS of the HUMAN FACTORS and ERGONOMICS SOCIETY 55th ANNUAL MEETING, NASA Ames Research Center, Moffett Field, CA USA, 2011.

[24] Lancraft R. Baron, S. and A. Caglayan. An Optimal Control Model Approach to the Design of Compensators for Simulator Delay. Scientific and Technical Information Branch, National Aeronautics and Space Administration, 1982. 
[25] J. G. Kimball Miller and D. R. Riley. The Effect of Visual-Motion Time Delays on Pilot Performance in a Pursuit Tracking Task. Tech.rep., NASA Langley Research Center, 1977.

[26] W. H. Levison and B. Papazian. The Effects of Time Delay and Simulator Mode on Closed-Loop Pilot/Vehicle Performance: Model Analysis and Manned Simulation Results. AIAA Flight Simulation Technologies, 1987.

[27] W. H. Levison and B. Papazian. The Effects of Asynchronous Visual Delays on Simulator Flight Performance and the Development of Simulator Sickness Symptomatology. Tech. rep., DTIC Document, 1986.

[28] J. De Maio. Visual Cueing Effectiveness: Comparison of Perception and Flying Performance. Fifth Interservice/Industry Training Equipment Conference, 1983.

[29] I. Owen S. Hodge G Barakos Y. Wang, M. White. Effects of visual and motion cues in flight simulation of ship-borne helicopter operations. Springer, The University of Liverpool, Liverpool L69 3GH, UK, 24 July 2013.

[30] P. K. Arban N. D. Macchiarella and S.M. Doherty. Transfer of Training from Flight Training Devices to Flight for Ab-Initio Pilots. International Journal of Applied Aviation Studies, Embry-Riddle Aeronautical University, Daytona Beach, Florida, 2006.

[31] Manual of Criteria for the Qualification of Flight Simulation Training Devices, Third Edition. INTERNATIONAL CIVIL AVIATION ORGANIZATION, Montral, Quebec, Canada, 2009.

[32] P. John Davison. A summary of studies conducted on the effect of motion in flight simulator pilot training. MPL Simulator Solutions, International Finance Centre, Hong Kong.

[33] Andrea L. Sparko J Brki-Cohen and Tiauw H. Go. Training Value of a FixedBase Flight Simulator with a Dynamic Seat. AIAA Modeling and Simulation Technologies Conference and Exhibit, Hilton Head, South Carolina, 2007.

[34] Online Oxford Dictionary - Parallax. http://www.oxforddictionaries.com/ definition/english/parallax. Accessed: December 2015.

[35] Wikipedia - Parallax. https://en.wikipedia.org/wiki/Parallax\#cite_ note-oed-2. Accessed: December 2015.

[36] Rockwell-Collins. https://www.rockwellcollins.com/. Accessed: 2015-11-16. 
[37] Microsoft FS Insider. http://www.microsoft.com/Products/Games/ FSInsider/news/Pages/AMessageFromAces . aspx. Accessed: 2015-11-16.

[38] Prepare3D. http://www.prepar3d.com/. Accessed: 2015-11-16.

[39] X-Plane. http://www.X-Plane.com/. Accessed: 2015-11-16.

[40] BenQ projectors. http://www.benq.ca/. Accessed: June 2015.

[41] Carl's Place. http://www. carlofet.com/. Accessed: June 2015.

[42] Kee Klamp. http://www. keeklamp.com/. Accessed: June 2015.

[43] Canada Flight Supplement. Nav Canada, Ottawa, 2014.

[44] X-Plane forum. http://forums.x-plane.org. Accessed: from June 2015 to September 2015.

[45] United States Geological Service. http://www.usgs.gov/. Accessed: June 2015.

[46] Southwestern Onatrio Orthophotography Project. http://www.ontario.ca/ page/land-information-ontario/. Accessed: June 2015.

[47] Open Street Map. http://www.ontario.ca/page/ land-information-ontario/. Accessed: June 2015.

[48] HD Mesh Scenery. http://www.alpilotx.net/. Accessed: June 2015.

[49] SketchUP. http://www.sketchup.com/. Accessed: July 2015.

[50] Immersive Display Pro. http://www.fly.elise-ng.net/ immersivedisplaypro. Accessed: June 2015.

[51] Audacity. http://audacityteam.org/. Accessed: November 2015.

[52] Benton L. Parris and Anthony M. Cook. Effects of visual and motion simulation cueing systems during takeoffs with engine failure. Technical report, NASA, 1978. 
Appendix A

Hardware and Software Specifications 


\section{$4 \times$ CURVS PC specs}

\section{System Information}

System Manufacturer:

BIOS:

Processor:

Memory:

Card name:

Manufacturer:

Chip type:

Display Memory:

Dedicated Memory:

Shared Memory:

Driver Model:

Driver File Version:

Operating System:

Language:

Simulator Software:

Edge-Blending Software:
ASUS

BIOS Date: 02/25/15 13:40:38 Ver: 23.03

Intel(R) Core(TM) i5-4690K CPU @ 3.50GHz (4 CPUs), 3.5GHz

16384MB RAM DDR5

\section{Display Devices}

NVIDIA GeForce GTX 960

NVIDIA

GeForce GTX 960

$4095 \mathrm{MB}$

$3072 \mathrm{MB}$

$1023 \mathrm{MB}$

WDDM 1.1

10.18.0013.5330 (English)

\section{Software}

Windows 7 Professional N 64-bit (6.1, Build 7601) Service Pack 1 (7601.win7sp1_gdr.151019-1254)

English (Regional Setting: English)

X-Plane 10.42

Immersive Display Pro v1.9 r1 


\section{Carleton University Redeveloped Vector Simulator (CURVS) System Architecture}

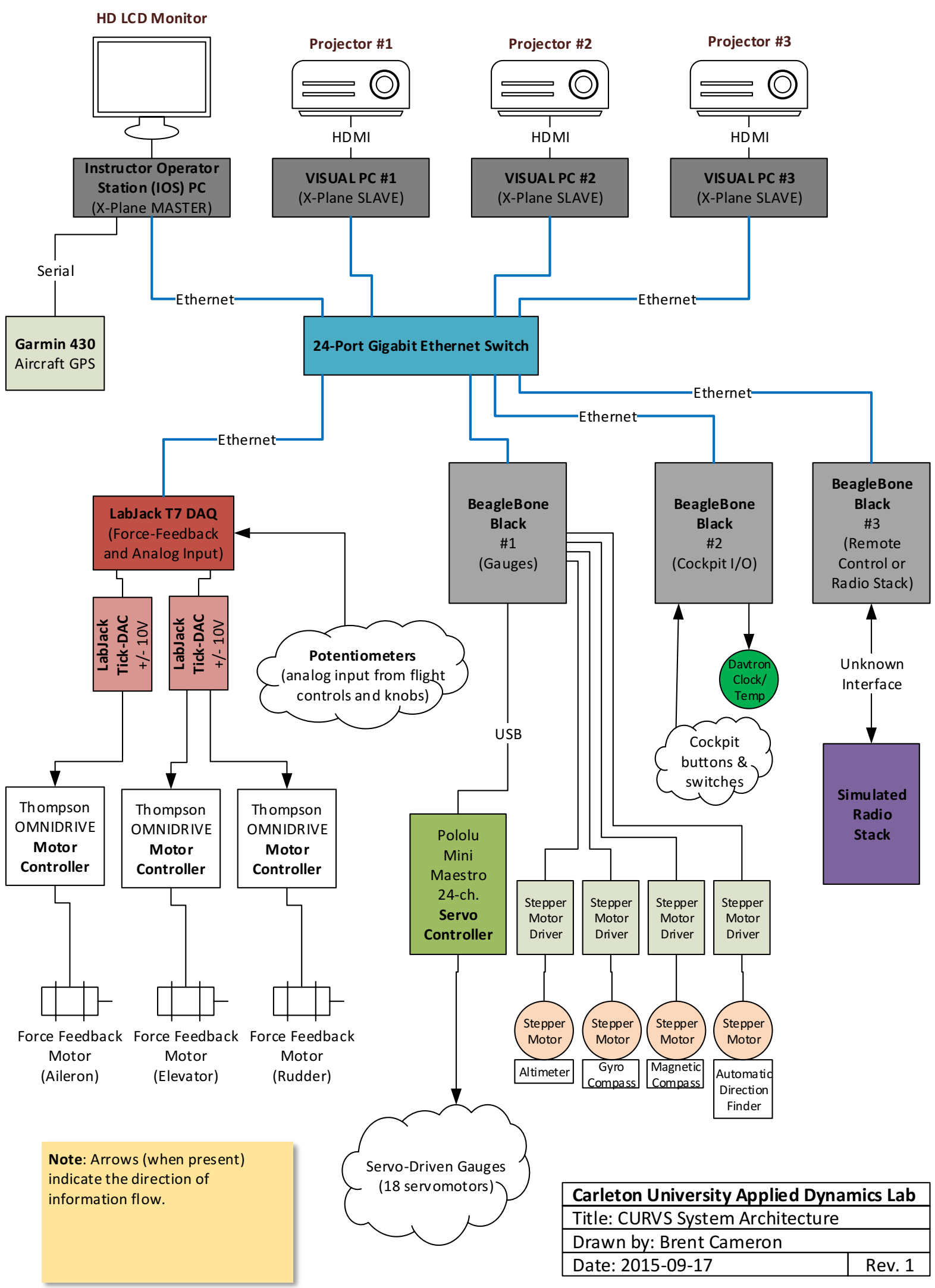




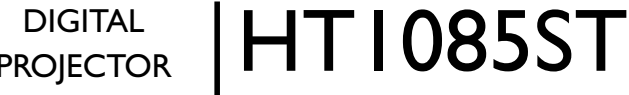

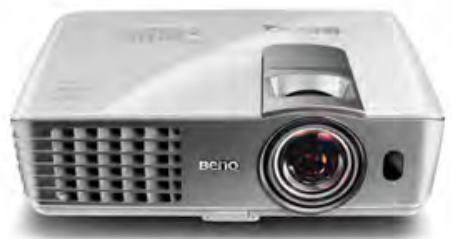

\section{Features}

- Native Full HD I080P (1920x 1080) Short Throw

2,200 ANSI Lumens, 10,000:I High Contrast Ratio

- Dual HDMI, MHL Connectivity

- OptionalWireless Full HD Kit

- 3D Full HD, built-in IOW speaker

- Horizontal \&Vertical Keystone

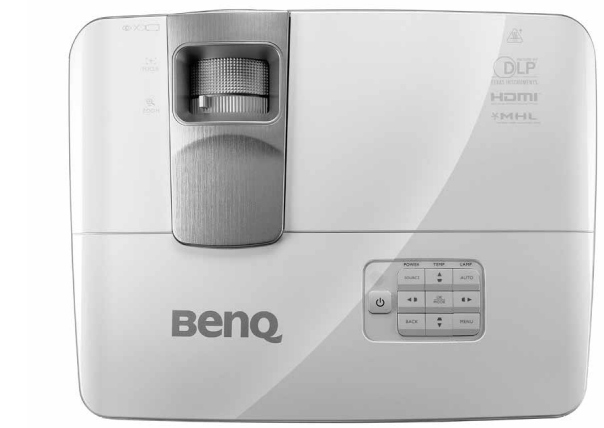

Input and Output Terminals

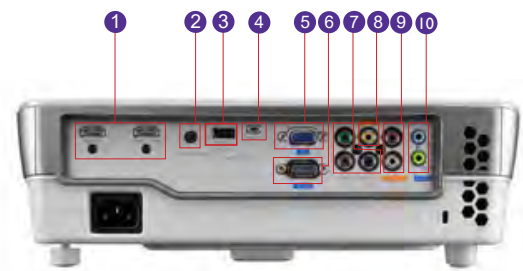

(1) HDMl $1 \& 2$ (MHL)

(2) DC 12V Trigger

(3) USB (TypeA)

(4) USB (Type Mini B)

(5) Computer in (D-sub 15pin)
6 RS232 (DB-9pin)

7 Component Video In (RCA)

8 S-Video In (Mini DIN 4pin)

(9) Audio in (Mini Jack)

(10) Audio LR in (RCA)

\section{isf}

\begin{tabular}{|c|c|c|}
\hline Projection System & \multicolumn{2}{|l|}{$\operatorname{DLP}^{\circledR}$} \\
\hline Native Resolution & \multicolumn{2}{|l|}{$1080 p(1920 \times 1080)$} \\
\hline Brightness & \multicolumn{2}{|c|}{2,200 ANSI lumens } \\
\hline Contrast Ratio & \multicolumn{2}{|c|}{$10,000: 1$} \\
\hline Display Color & \multicolumn{2}{|c|}{1.07 Billion Colors } \\
\hline Lens & \multicolumn{2}{|c|}{$\mathrm{F}=2.6-2.78, \mathrm{f}=10.2-12.24 \mathrm{~mm}$} \\
\hline Aspect Ratio & \multicolumn{2}{|c|}{ Native 16:9 (5 aspect ratio selectable) } \\
\hline Throw Ratio & \multicolumn{2}{|c|}{ 0.69-0.83 (65.7"@ 1M) } \\
\hline Image Size (Diagonal) & \multicolumn{2}{|c|}{$38 "-300 "$} \\
\hline Zoom Ratio & \multicolumn{2}{|l|}{$1.2: 1$} \\
\hline Lamp Type & \multicolumn{2}{|l|}{$240 \mathrm{~W}$} \\
\hline Lamp (Normal/Economic Mode) & \multicolumn{2}{|c|}{$3500 / 5000 / 6000$ hours } \\
\hline Keystone Adjustment & \multicolumn{2}{|c|}{ 2D, Vertical \& Horizontal $+/-20$ degrees } \\
\hline Projection Offset & \multicolumn{2}{|c|}{ Vertical $105 \% \sim \pm 5 \%$} \\
\hline Resolution Support & \multicolumn{2}{|l|}{ up to WUXGA } \\
\hline Horizontal Frequency & \multicolumn{2}{|l|}{$31 \mathrm{~K}-102 \mathrm{KHz}$} \\
\hline Vertical Scan Rate & \multicolumn{2}{|l|}{$23-120 \mathrm{~Hz}$} \\
\hline Compatibility & \multicolumn{2}{|c|}{$\begin{array}{l}\text { SDTV, EDTV, HDTV Compatibility: 480i, 480p, 576i, 576p, 720p, 1080i, 1080p } \\
\text { Video Compatibility: NTSC, PAL, SECAM }\end{array}$} \\
\hline Interface & $\begin{array}{l}\text { Computer in (D-sub } \\
\text { HDMl } \\
\text { MHL } \\
\text { Composite Video in } \\
\text { Component Video in } \\
\text { Audio in (Mini Jack) } \\
\text { Audio L/R in (RCA) } \\
\text { Audio out (Mini Jack } \\
\text { Chamber Speaker } \\
\text { USB (Type A) } \\
\text { USB (Type Mini B) } \\
\text { RS232 (DB-9pin) } \\
\text { DC 12V Trigger }\end{array}$ & $\begin{array}{l}x 1 \\
\times 2 \\
\times 1 \text { (shared with } \mathrm{HDMl}-2) \\
\times 1 \\
\times 1 \\
\times 1 \\
\times 1 \\
\times 1 \\
10 \mathrm{~W} \times 1 \\
\times 1 \text { (for } 1.5 \mathrm{~V} \text { power supply) } \\
\times 1 \text { (for service) } \\
\times 1 \\
\times 1\end{array}$ \\
\hline Dimensions $(\mathrm{WxH} \times \mathrm{D})$ & $12.2^{\prime \prime} \times 4.09^{\prime \prime} \times 9.6^{\prime \prime}$ & \\
\hline Weight & $6.28 \mathrm{lbs}$ & \\
\hline Power Supply & AC 100 to $240 \mathrm{~V}, 50$ & $\mathrm{~Hz}$ \\
\hline Power Consumption & 353W/110V (typical) & ndby $<0.5 \mathrm{~W}$ \\
\hline Audible Noise & $31 / 28 \mathrm{dBA}$ (Normal// & omic mode) \\
\hline $\begin{array}{l}\text { On-Screen Display } \\
\text { Languages }\end{array}$ & $\begin{array}{l}\text { Arabic/Bulgarian/ } \\
\text { French/ German/ } \\
\text { Japanese/ Korean } \\
\text { Russian/ Simplifie } \\
\text { Traditional Chines }\end{array}$ & $\begin{array}{l}\text { tian/ Czech/ Danish/ Dutch/ English/ Finnish/ } \\
\text { k/ Hindi/ Hungarian/ Italian/ Indonesian/ } \\
\text { rwegian/ Polish/ Portuguese/ Romanian/ } \\
\text { inese/Spanish/ Swedish/ Turkish/ Thai/ } \\
3 \text { Languages) }\end{array}$ \\
\hline Preset Modes & $\begin{array}{l}\text { ISF Disabled: Brigh } \\
\text { ISF Disabled: Bright/s }\end{array}$ & $\begin{array}{l}\text { andard)/Cinema/User } 1 \text { / User } 2 \\
\text { lard/Cinema/User 1/User 2/ (ISF Night)/ ISF Day }\end{array}$ \\
\hline Accessories (Standard) & $\begin{array}{l}\text { Remote Control w/ } \\
\text { Quick Start Guide, }\end{array}$ & $\begin{array}{l}\text { ery, Power Cord, User Manual CD, Lens Cover, } \\
\text { ranty Card, VGA (D-sub 15pin) Cable }\end{array}$ \\
\hline Accessories (Optional) & $\begin{array}{l}\text { Spare Lamp Kit } \\
\text { Ceilling Mount } \\
\text { 3D Glasses } \\
\text { Carry Bag } \\
\text { Wireless FHD Kit }\end{array}$ & $\begin{array}{l}\text { P/N: 5J.J9H05.001 } \\
\text { P/N: 5J.J4N10.001 } \\
\text { P/N: 5J.J7L25.002 } \\
\text { P/N: 5J.J3T09.001 } \\
\text { P/N: 5J.J9H28.A01 }\end{array}$ \\
\hline Limited Warranty & 1 Year Parts/Labo & 0day or 500 hours of lamp life. \\
\hline
\end{tabular}

"Lamp life results will vary depending on environmental conditions and usage. Actual product's features and specifications are subject to change without notice.

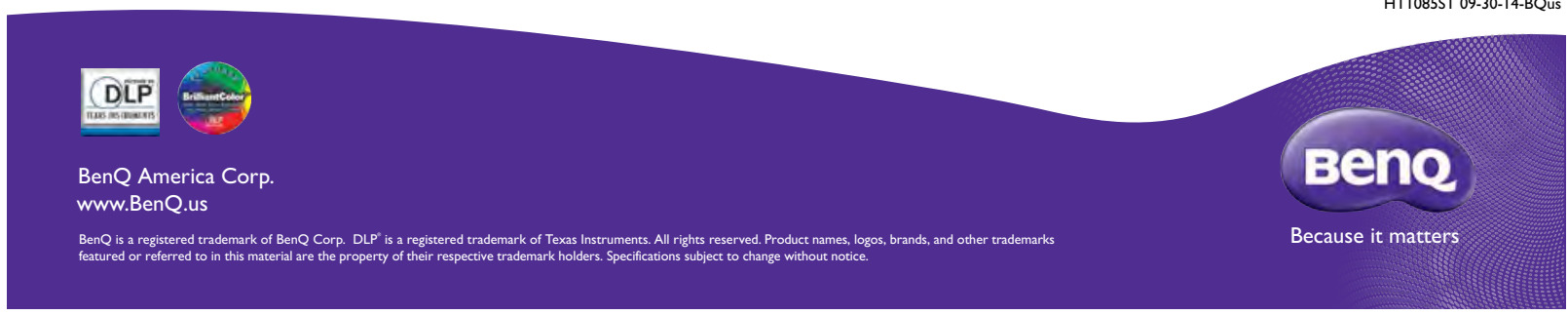




\section{HTI085ST Digital Projector}

\section{Distance Chart}

Floor Installation

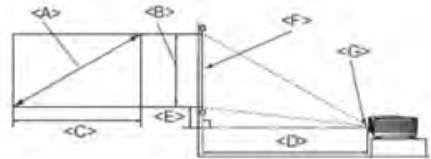

$<\mathrm{F}>$ : Screen

$<\mathrm{G}>$ :Center of lens

\section{Ceiling Installation}

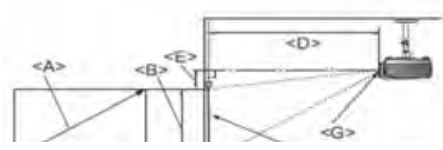

$<\mathrm{F}>$ : Screen
The screen aspect ratio is $16: 9$ and the projected picture is $16: 9$

\begin{tabular}{|c|c|c|c|c|c|c|c|}
\hline \multicolumn{4}{|c|}{ Screen Dimensions } & \multicolumn{3}{|c|}{ Projection Distance $\langle D>$ (inch) } & \multirow{3}{*}{$\begin{array}{l}\text { Lowest/Highest } \\
\text { lens position } \\
<E>\text { (inch) }\end{array}$} \\
\hline \multicolumn{2}{|c|}{ Diagonal $\langle A\rangle$} & \multirow{2}{*}{$\begin{array}{l}\text { Height }<B> \\
\text { (inch) }\end{array}$} & \multirow{2}{*}{$\begin{array}{c}\text { Width }<C> \\
\text { (inch) }\end{array}$} & \multirow{2}{*}{\begin{tabular}{|c|} 
Min Distance \\
(with max zoom) \\
\end{tabular}} & \multirow{2}{*}{ Average } & \multirow{2}{*}{\begin{tabular}{|c|} 
Max Distance \\
(with min zoom)
\end{tabular}} & \\
\hline Inch & $\mathrm{mm}$ & & & & & & \\
\hline 30 & 762 & 15 & 26 & 18 & 20 & 22 & .35 \\
\hline 40 & 1016 & 20 & 35 & 24 & 26 & 28 & .47 \\
\hline 50 & 1270 & 25 & 44 & 30 & 33 & 36 & .62 \\
\hline 60 & 1524 & 30 & 52 & 36 & 40 & 43 & .74 \\
\hline 70 & 1778 & 34 & 61 & 42 & 46 & 50 & .86 \\
\hline 80 & 2032 & 39 & 70 & 48 & 53 & 58 & .98 \\
\hline 90 & 2286 & 44 & 78 & 54 & 60 & 65 & 1.1 \\
\hline 100 & 2540 & 49 & 87 & 60 & 66 & 72 & 1.2 \\
\hline 110 & 2794 & 54 & 96 & 66 & 73 & 80 & 1.3 \\
\hline 120 & 3048 & 59 & 104 & 72 & 80 & 86 & 1.4 \\
\hline 130 & 3302 & 64 & 113 & 78 & 86 & 94 & 1.6 \\
\hline 140 & 3556 & 68 & 122 & 84 & 92 & 101 & 1.7 \\
\hline 150 & 3810 & 74 & 130 & 90 & 98 & 108 & 1.8 \\
\hline 160 & 4064 & 78 & 140 & 96 & 105 & 115 & 1.9 \\
\hline 170 & 4318 & 83 & 148 & 101 & 112 & 122 & 2.1 \\
\hline 180 & 4572 & 88 & 157 & 108 & 119 & 129 & 2.2 \\
\hline 190 & 4826 & 93 & 165 & 114 & 125 & 137 & 2.3 \\
\hline 200 & 5080 & 98 & 174 & 120 & 132 & 144 & 2.4 \\
\hline 250 & 6350 & 122 & 218 & 150 & 164 & 180 & 3.1 \\
\hline 300 & 7620 & 147 & 261 & 180 & 198 & 216 & 3.7 \\
\hline
\end{tabular}

\section{Ceiling Mount Chart}

Ceiling Mount Screws: M4 (Max. L = 25 mm, Min. $L=20 \mathrm{~mm}$ )
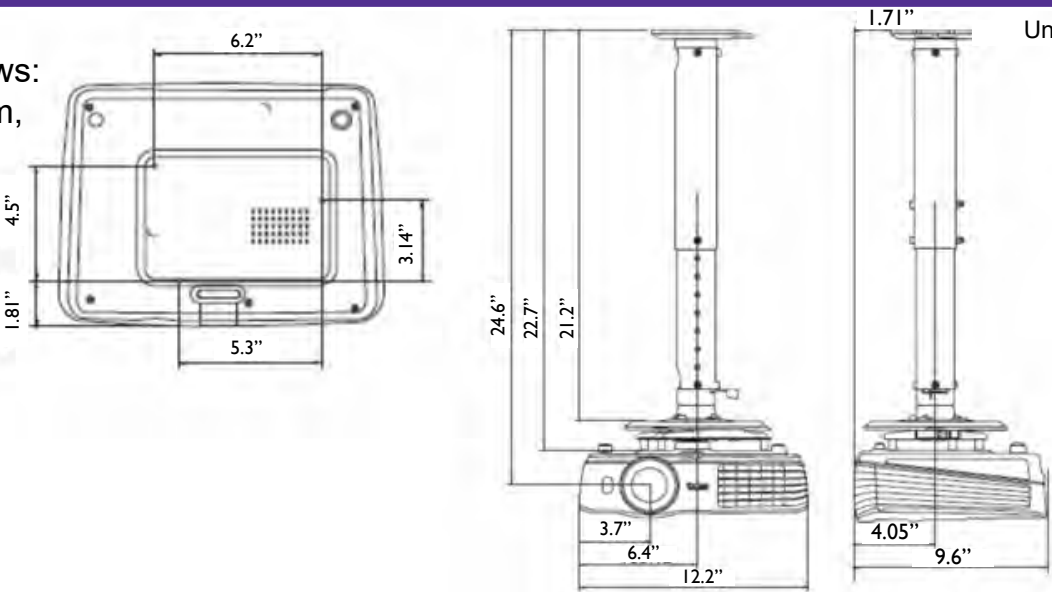

\section{Dimensions}
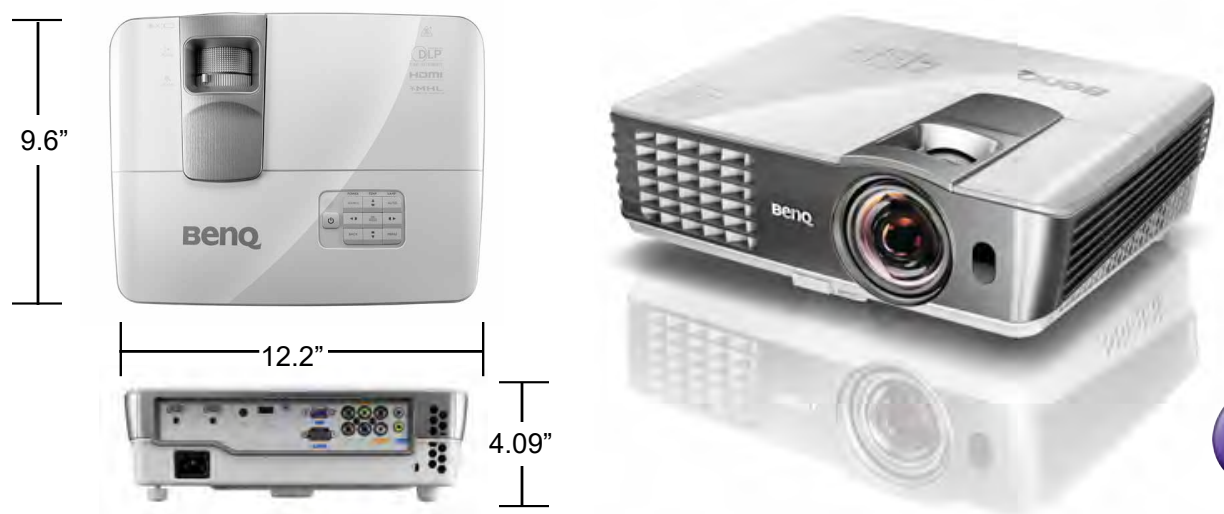

Units: inch

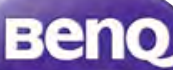


Appendix B

Fabric Factsheet and the Screen Tubes 


\begin{tabular}{|c|c|c|}
\hline Item & Sku & Qty \\
\hline Custom: Finished Edges with & custom- & 1 \\
\hline Grommets & finished- & \\
\hline Size & edge & \\
\hline \multicolumn{3}{|l|}{$82.67 \times 317.32$ inch } \\
\hline \multicolumn{3}{|l|}{ Size Specifics } \\
\hline \multicolumn{3}{|l|}{$* *$ Viewable** Dimensions } \\
\hline \multicolumn{3}{|l|}{ Material } \\
\hline \multicolumn{3}{|l|}{ FlexiWhite } \\
\hline \multicolumn{3}{|l|}{ Notes: } \\
\hline \multicolumn{3}{|l|}{$87.67 \times 322.32$ inch Outer } \\
\hline \multicolumn{3}{|l|}{ Dimensions } \\
\hline 6" Black Ball Bungees - For Each & BBB-6 & 75 \\
\hline Finished Edge Projection Screen & & \\
\hline Grommet Hole & & \\
\hline
\end{tabular}



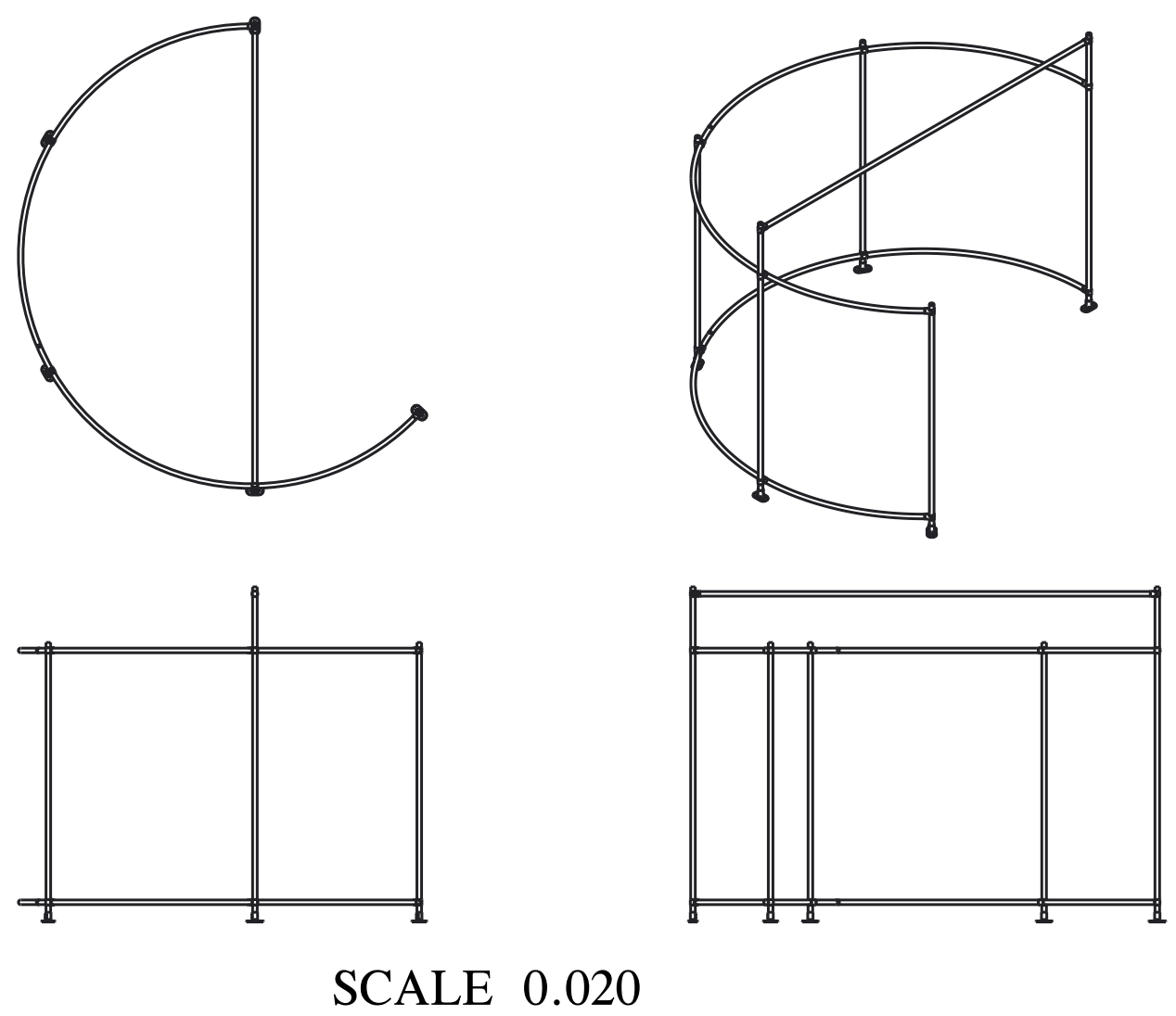
4 Units at

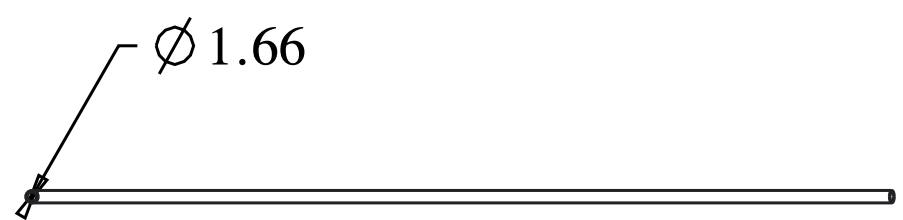

Inches

SCALE 0.050

$\mathrm{R} 82.05$

Note: Bend length 163" 


\section{Unit at}

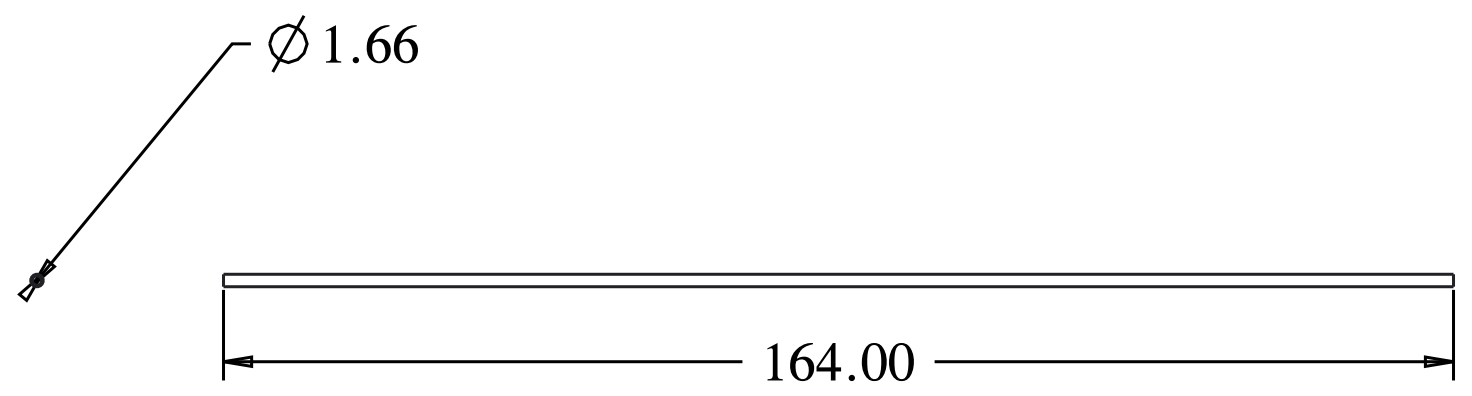

Inches

SCALE 0.050 


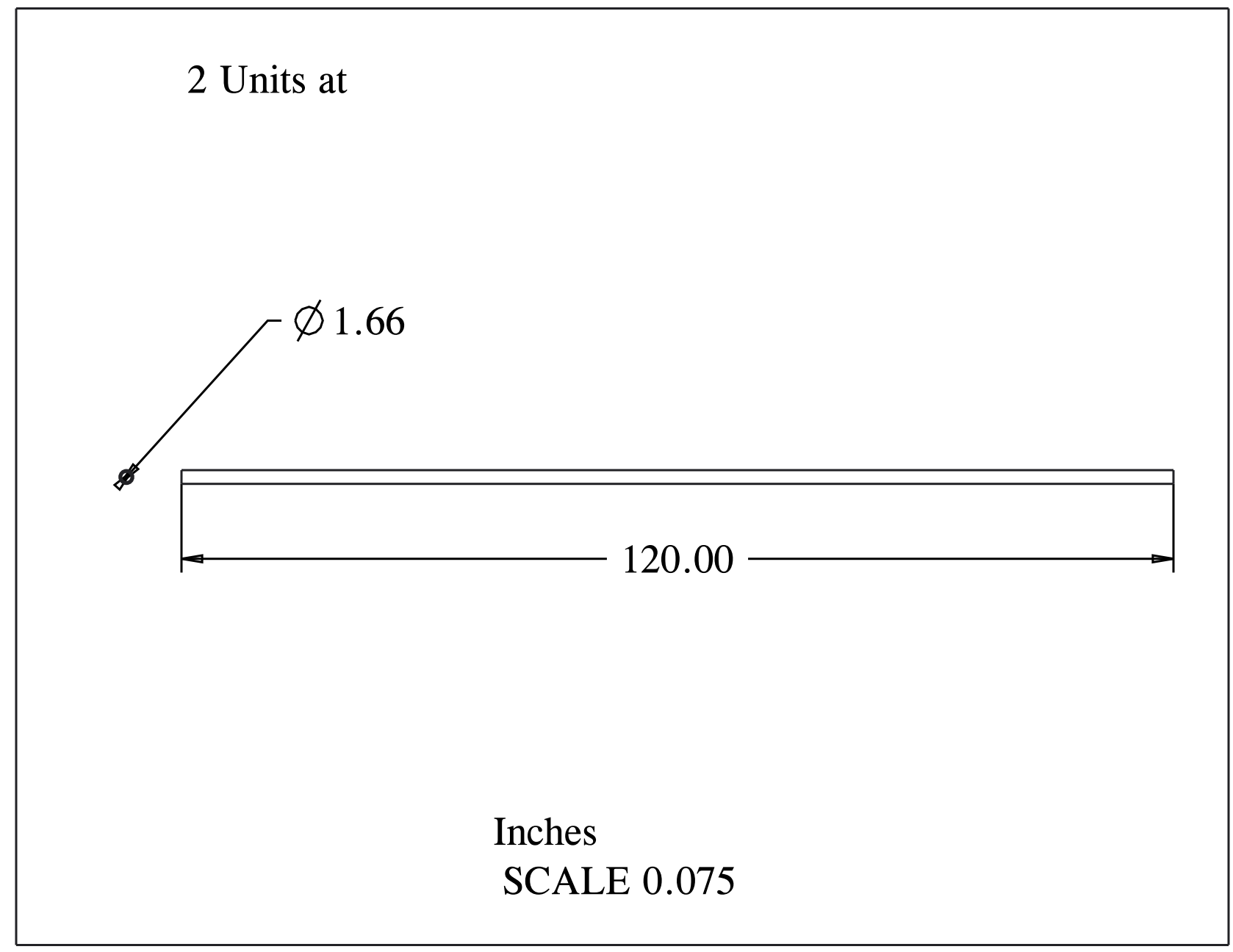


3 Units at

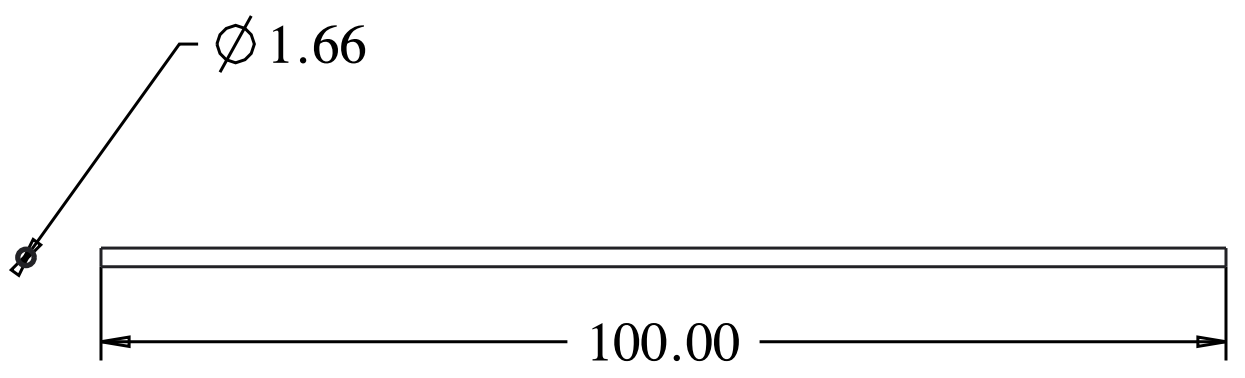

Inches

SCALE 0.075 


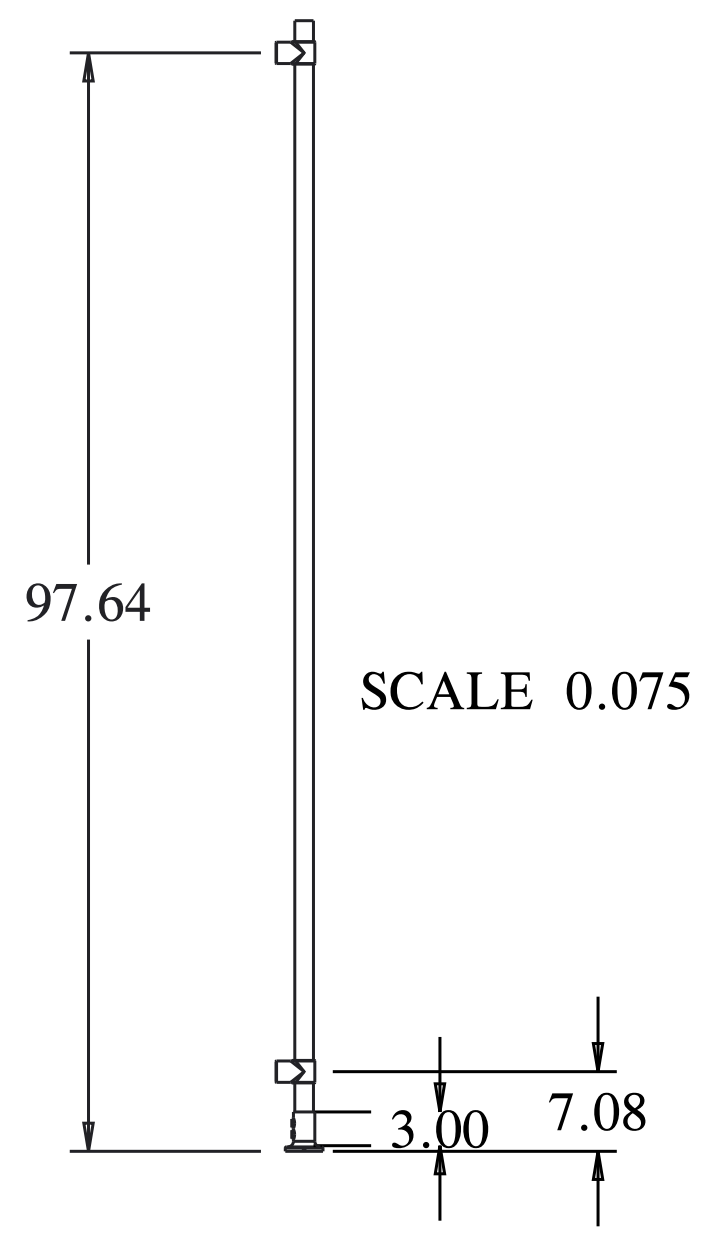




\section{Appendix $\mathrm{C}$}

\section{Immersive Calibration Pro Properties}


Calculated X-Plane input angles (output from the immersive calibration pro):

\begin{tabular}{|c|c|}
\hline $\begin{array}{l}\text { X-Plane Viewing } \\
\text { Angles }\end{array}$ & $\begin{array}{c}\text { Number of Columns and Rows in Immersive } \\
\text { Calibration Pro }\end{array}$ \\
\hline $\begin{array}{r}\text { Projector } 1 \text { (0 } 0 \text { 1920 1080@VectorSim-Vis1) } \\
\text { LateralFOV }=85.57 \\
\text { LateralOffset }=-88.11 \\
\text { VerticalOffset }=-4.70 \\
\text { Projector } 2\left(\begin{array}{lll}0 & 0 & 19201080 @ \text { VectorSim-Vis2) }\end{array}\right. \\
\text { LateralFOV }=89.16 \\
\text { LateralOffset }=-20.00 \\
\text { VerticalOffset }=-3.60 \\
\text { Projector } 3(0 \quad 0 \quad 19201080 @ \text { VectorSim-Vis3) } \\
\text { LateralFOV }=86.78 \\
\text { LateralOffset }=47.17 \\
\text { VerticalOffset }=-4.00\end{array}$ & $\begin{array}{c}\text { Column: } 21 \\
\text { Rows: } 6 \\
\text { Number of Views: } 3 \\
\text { Column Length: } 0.384 \mathrm{~m} \\
\text { Row Length: } 0.346 \mathrm{~m} \\
\text { Begin Angle: }-130 \\
\text { End Angle: } 90\end{array}$ \\
\hline
\end{tabular}

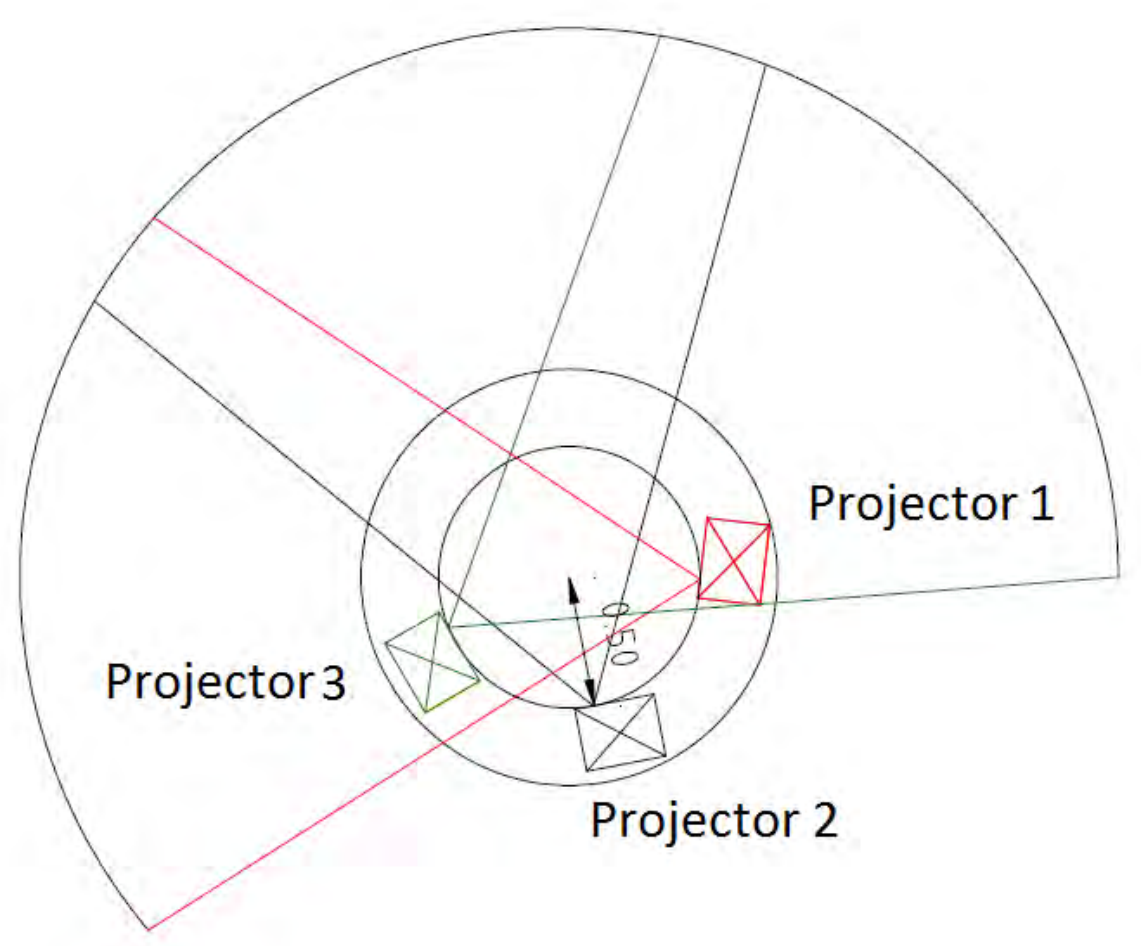


Appendix D

Flight Tests Weather Data 


\section{Flight Test 1: METAR Data}

\section{June 07,2015}

\section{Station: Gatineau_Airpor QB CN CYND 545.52 -75.57 6499999148}

METAR CYND 070000Z 30003KT 15SM BKN220 16/05 A3014 RMK CI6 SLP206

METAR CYND 070100Z 34005KT 15SM SCT220 13/05 A3013 RMK CI4 LAST OBS/NEXT 071100 UTC SLP205

METAR CYND 071100Z 02003KT 15SM BKN260 08/05 A3015 RMK CI7 SLP210

METAR CYND 071200Z 10004KT 15SM BKN260 11/06 A3013 RMK CI7 SLP205

METAR CYND 071300Z VRB02KT 15SM BKN250 13/07 A3014 RMK CI7 SLP208

METAR CYND $071400 Z$ VRB04KT 15SM FEW140 OVC240 16/07 A3012 RMK AC1CI7 SLP201

METAR CYND 071500Z 11003KT 050V160 15SM SCT140 OVC220 18/08 A3010 RMK AC3CI5 SLP194 DENSITY ALT 400FT

METAR CYND 071600Z 15009KT 110V180 15SM FEW140 OVC210 19/04 A3007 RMK AC2CS6 SLP185 DENSITY ALT 500FT

METAR CYND 071700Z 15007KT 130V200 15SM BKN150 BKN220 20/06 A3004 RMK AC5CI2 PRESER SLP174 DENSITY ALT 600FT

METAR CYND 071800Z 16007KT 130V230 15SM SCT150 BKN220 20/06 A3002 RMK AC4CI3 SLP167 DENSITY ALT 700FT

METAR CYND 071900Z 16006KT 120V200 15SM SCT150 OVC220 22/09 A2999 RMK AC4CI4 SLP156 DENSTTY ALT 900FT

METAR CYND 072000Z 16008KT 15SM BKN150 OVC220 22/08 A2996 RMK AC5CI3 SLP148 DENSITY ALT $1000 \mathrm{FT}$

METAR CYND 072100Z 13009KT 15SM FEW150 BKN220 22/08 A2993 RMK AC2CI5 SLP137 DENSITY ALT 1100FT

METAR CYND 072200Z 16011KT 15SM FEW150 BKN220 23/10 A2989 RMK AC2CI5 SLP123 DENSITY ALT $1200 \mathrm{FT}$

METAR CYND 072300Z 16005KT 15SM BKN180 OVC220 22/08 A2987 RMK AC5CI3 SLP115 DENSITY ALT 1100FT 
Flight Test 2: METAR Data

August 08, 2015

Station: Gatineau_Airpor QB CN CYND 545.52 -75.57 6499999148

METAR CYND 080000Z 04004KT 15SM FEW075 FEW230 20/14 A3000 RMK AC1CI1 SLP158 DENSITY ALT 700FT

METAR CYND 080100Z 01004KT 15SM FEW080 FEW240 15/13 A3000 RMK AC1CI2 AC TR LAST OBS/NEXT 081100 UTC SLP161

METAR CYND 081100Z 00000KT 15SM FEW120 FEW250 11/11 A3006 RMK AC1CI1 SLP181

METAR CYND 081200Z 00000KT 15SM FEW100 SCT260 15/14 A3007 RMK AC1CI2 SLP183

METAR CYND 081300Z VRB03KT 15SM FEW100 BKN260 18/15 A3007 RMK AC1CI5 SLP183 DENSITY ALT 300FT

METAR CYND 081400Z VRB02KT 15SM FEW035 BKN260 19/14 A3007 RMK CU1CI6 SLP184 DENSITY ALT 500FT

METAR CYND 081500Z 13004KT 010V150 15SM BKN027 BKN260 20/13 A3008 RMK CU6CI1 MDT CU ASOCTD SLP186 DENSITY ALT 600FT

METAR CYND 081600Z 00000KT 15SM BKN032 BKN260 22/15 A3008 RMK CU5CI2 MDT CU ASOCTD SLP186 DENSITY ALT 800FT

METAR CYND 081700Z VRB03KT 15SM SCT040 BKN260 23/12 A3007 RMK CU3CI3 SLP183 DENSITY ALT 900FT

METAR CYND 081800Z 00000KT 15SM SCT050 SCT080 BKN260 23/11 A3007 RMK CU3AC1CI2 SLP183 DENSITY ALT 1000FT

METAR CYND 081900Z 15005KT 030V170 15SM FEW050 SCT080 BKN260 23/12 A3006 RMK CU2AC2CI2 SLP181 DENSITY ALT 1000FT

METAR CYND 082000Z VRB03KT 15SM FEW050 SCT080 BKN260 24/12 A3005 RMK CU2AC1CI3 SLP177 DENSITY ALT 1100FT

METAR CYND 082100Z 02006KT 360V080 15SM FEW045 SCT080 BKN260 23/13 A3005 RMK CU2AC2CI2 SLP177 DENSITY ALT 1000FT

METAR CYND 082200Z VRB02KT 15SM FEW045 SCT080 BKN260 24/13 A3005 RMK CU2AC1CI3 SLP175 DENSITY ALT 1100FT

METAR CYND 082300Z VRB05KT 15SM FEW045 SCT070 BKN260 22/13 A3005 RMK CU1AC2CI2 SLP177 DENSITY ALT 900FT

http://vortex.plymouth.edu/myo/sfc/textobs-a.html 


\section{Flight Test 3: METAR Data}

September 25,2015

\section{Station: Gatineau_Airpor QB CN CYND 545.52 -75.57 6499999148}

METAR CYND 250000Z 36006KT 15SM BKN160 BKN240 15/07 A3044 RMK AC5CI1 $\operatorname{SLP} 308$

METAR CYND $250100 Z$ 36007KT 15SM SCT160 BKN240 14/07 A3044 RMK AC3CI3 LAST OBS/NEXT 251100 UTC SLP309

METAR CYND $251100 Z$ 04005KT 15SM SCT080 BKN170 11/05 A3047 RMK AC3AC4 SLP 318

METAR CYND 251200Z 04005KT 15SM FEW080 BKN170 11/05 A3048 RMK AC1AC6 $\operatorname{SLP} 323$

METAR CYND 251300Z 05007KT 15SM FEW080 BKN170 12/04 A3049 RMK AC1AC6 SLP326

METAR CYND $251400 Z$ 06007KT 030V090 15SM FEW080 BKN160 13/04 A3051 RMK AC1AC6 SLP331

METAR CYND 251500Z 07009KT 15SM FEW080 BKN160 14/03 A3049 RMK AC1AC6 $\operatorname{SLP} 327$

METAR CYND 251600Z 08008KT 15SM FEW080 OVC160 15/04 A3049 RMK AC1AC7 SLP326

METAR CYND 251700Z 12005KT 080V150 15SM SCT140 BKN150 15/05 A3046 RMK AC3AC4 SLP315

METAR CYND 251800Z 09005KT 060V160 15SM BKN140 15/06 A3045 RMK AC7 SLP312

METAR CYND 251900Z 04008KT 360V070 15SM BKN140 15/04 A3045 RMK AC7 SLP311

METAR CYND 252000Z 03007KT 340V050 15SM BKN100 15/04 A3043 RMK AC7 SLP307

METAR CYND 252100Z 35005KT 330V050 15SM BKN100 15/06 A3043 RMK AC7 SLP306

METAR CYND 252200Z 33003KT 15SM BKN090 15/07 A3044 RMK AC6 SLP308

METAR CYND 252300Z 34005KT 15SM FEW090 11/07 A3044 RMK AC2 SLP310

http://vortex.plymouth.edu/myo/sfc/textobs-a.html 


\section{Appendix E}

\section{Instrument Specifications}

Accelerometer, Spot Meter, Sound Pressure Level, and Sound Recorder 


\section{Gulf Coast Data [oncepts \\ www.gcdataconcepts.com}

USB Accelerometer Model X16-1D

\section{Features}

- 3-axis $\pm 16 \mathrm{~g}$ accelerometer

- 16-bit resolution

- User selectable sample rate of 12,25 , 50, 100, 200, 400 Hertz

- Finite Impulse Response filter

- Accurate time stamped data using Real Time Clock (RTC)

- Convenient on/off button

- Data recorded to a removable microSD card (8GB included)

- Easily readable comma separated text data files

- Data transfer compatible with Windows or Linux via Universal Serial Bus (USB) interface (no special software)

- System appears as USB Mass Storage Device to Windows and Linux OS's.

- Standard replaceable "AA" type battery

- LED indicator lights for system status

- Weighs $20 z(55 \mathrm{~g})$ with alkaline battery

\section{Applications}

The X16-1D is applicable to:

- Monitoring human motor activity, or actigraphy, such as exercise intensity or sleeping disorders.

- Automotive performance monitoring

- Educational purposes

\section{Description}

The USB Accelerometer X16-1D uses a low noise digital accelerometer sensor, precise time stamped data logging, microSD memory storage, real-time data access and USB connectivity. Acceleration is collected in X, Y, and $Z$ axes and stored at a user selectable rate of up to $400 \mathrm{hz}$. When connected via the USB to a personal computer, the X16-1D appears as a standard mass storage device containing the comma delimited data files and user setup files. The X16-1D operates from a replaceable AA type alkaline battery

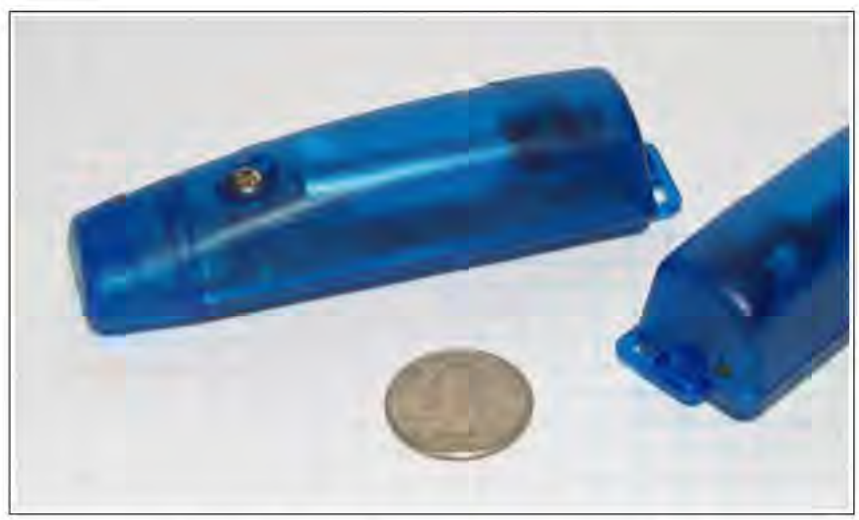

Figure 1: USB Accelerometer X16-1D 
USB Accelerometer Model X16-1D

Table 2: Accelerometer Sensor Characteristics

\begin{tabular}{|c|c|c|c|c|c|}
\hline Parameter & Condition & Min & Typical & Max & Units \\
\hline Acceleration range & & & \pm 16.0 & & $\mathrm{~g}$ \\
\hline Sensitivity & & & 2048 & & $\mathrm{count} / \mathrm{g}$ \\
\hline Sensitivity Deviation & & & \pm 1.0 & & $\%$ \\
\hline Nonlinearity & $\mathrm{X}, \mathrm{Y}, \mathrm{Z}$ axis & & \pm 0.5 & & $\% \mathrm{FS}$ \\
\hline $\begin{array}{c}\text { Zero-g Offset Level } \\
\text { Accuracy }\end{array}$ & $\mathrm{X}, \mathrm{Y}$ axis & -150 & & +150 & $\mathrm{mg}$ \\
\cline { 2 - 6 } & $\mathrm{Z}$ axis & -250 & & +250 & $\mathrm{mg}$ \\
\hline Inter-Axis Alignment Error & & & \pm 0.1 & & Degrees \\
\hline Cross-Axis Sensitivity & & & \pm 1 & & $\%$ \\
\hline
\end{tabular}
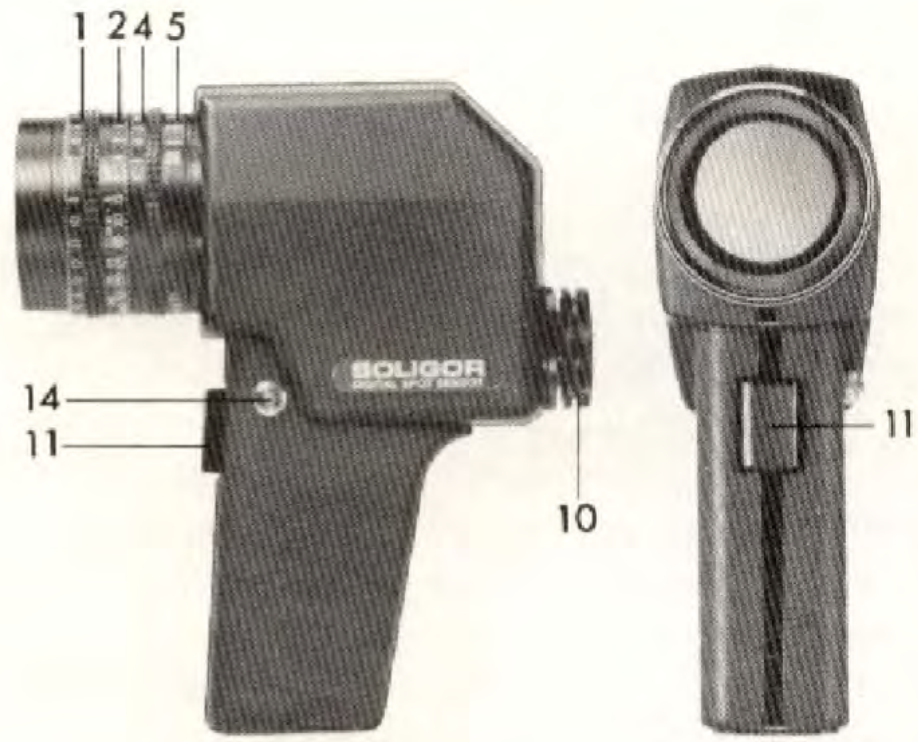

\section{80}
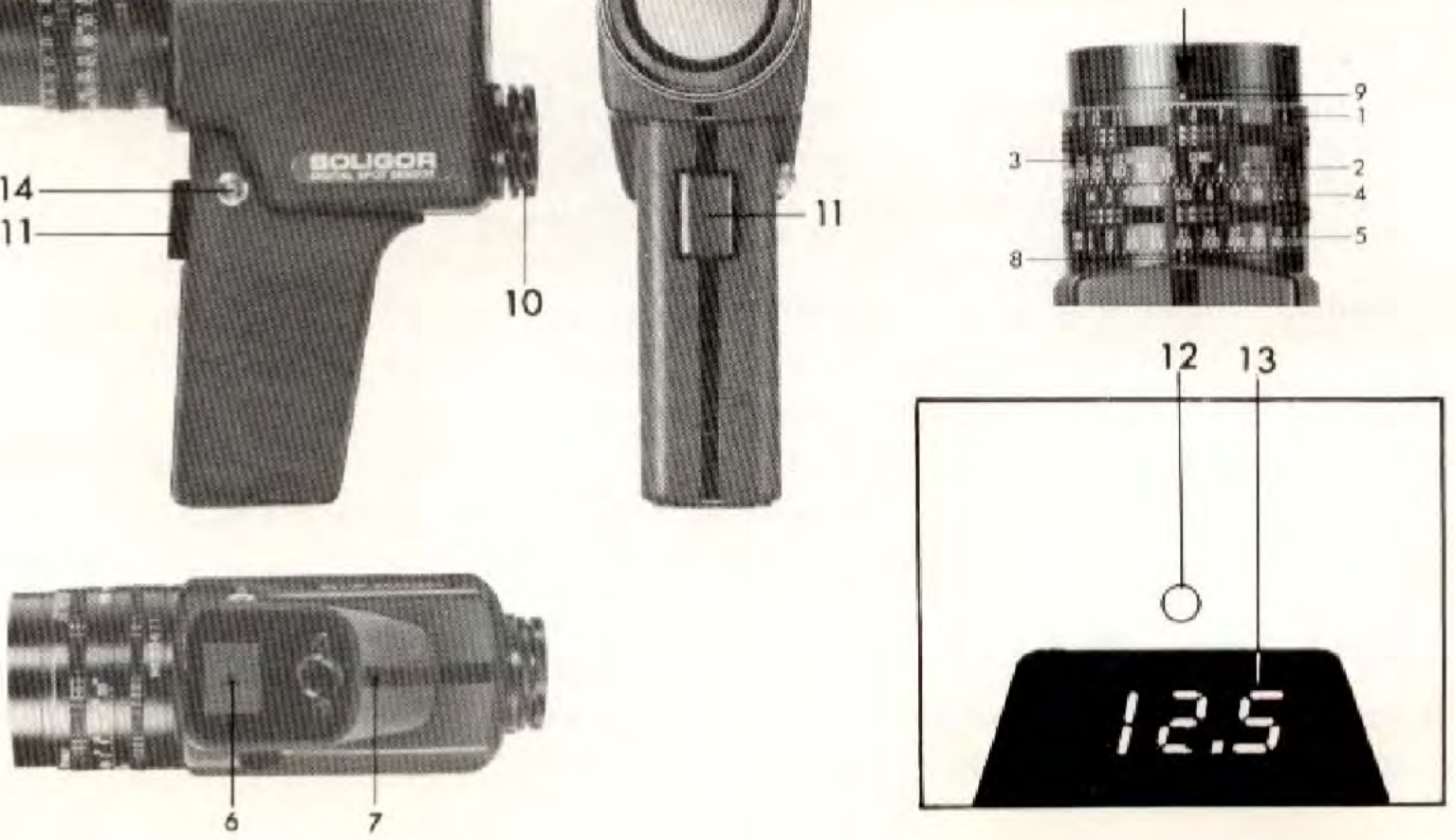


\section{Specifications}

Angle of incidence: $\quad 1^{\circ}$

Angle of view, horizontal: $15^{\circ}$

Angle of view, vertical: $\quad 11^{\circ}$

Viewfinder:

Diopter adjustment system Pentaprism and condenser

EV-range:

ASA-range:

1 19.9 (ASA 100) in one range

$6-12,800($ DIN $9-42)$

Shutter speeds:

$1 / 4000 \sim 30 \mathrm{~min}$.

Cine speeds:

$8-18-32-64-128$ fps

Lens opening;

$F: 1 \sim F: 128$

Batteries:

1 pc 9 volt Dry battery Type 006P (Eveready 522 or Mallory MN1604).

Measuring method: Direct reading system

Lens:

$100 \mathrm{~mm}$ f2.8, Fully coated.

\section{Accessories}

Soligor offers a complete range of accessories for your Digital Spot Sensor Meter,

Rubber lens hood: Soligor $43 \mathrm{~mm}$ Rubber Lens Hood

Filters: Choose from a wide range of Soligor $43 \mathrm{~mm}$ filters for black \& white and color films. Additional Soligor filters are also available for fluorescent light and polarizing.

\section{Exposure Value Conversion Table}

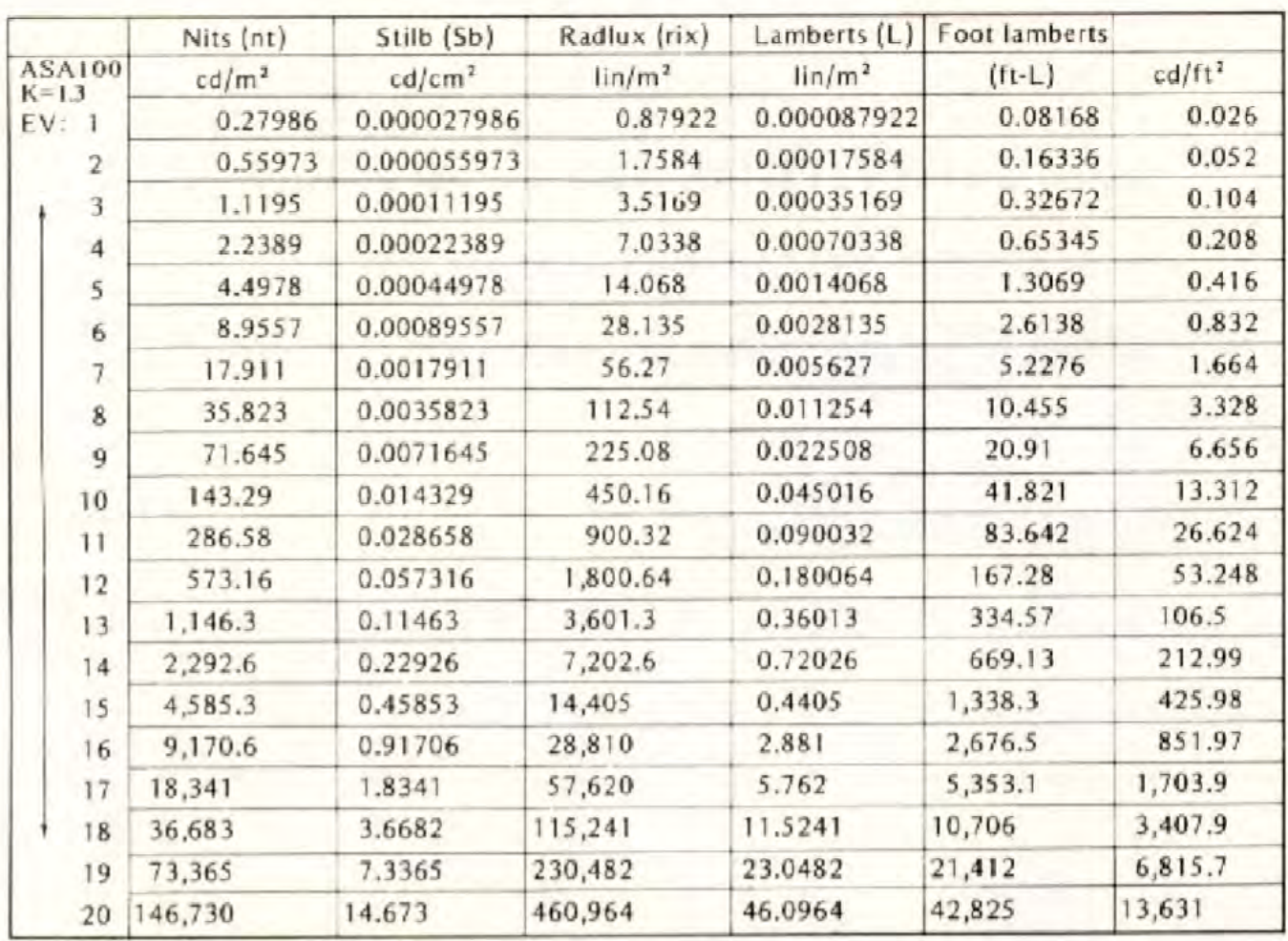




\section{SOUND LEVEL METER}

\section{Sound Level Meter GM1351}

*This unit was designed according to following standards:

\section{GM1351}

-.--A. International electrician committee standard: IEC PUB 651

TYPE2

---B. US national standard: ANSI S1.4 TYPE2

*Measuring range: $30 \sim 130 \mathrm{dBA}$

*Accuracy: $\pm 1.5 \mathrm{~dB}$

*Digit \& Resolution: 4 digits \& $0.1 \mathrm{~dB}$

*Frequency response: $31.5 \mathrm{~Hz} \sim 8.5 \mathrm{KHz}$

* Frequency weighting characteristic: $\mathrm{A}$

*Sampling rate: 2times/second

*Microphone: 1/2 inch polarization capacitance microphone

*Max reading

${ }^{*}$ Automatic backlight

*Low battery indication

*Automatic power off

*Packing method: Blister + Card

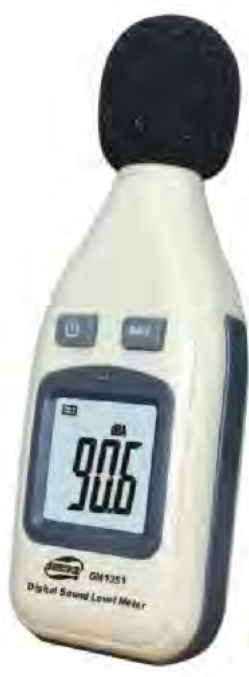

*Power: $9 \mathrm{~V}$ battery (6F22)

*Dimension: $57^{*} 36 * 149 \mathrm{~mm}$

*Weight: $144 \mathrm{~g}$ (including battery)

Range: 30 130dBA

Accuracy: $\pm 1.5 \mathrm{~dB}$ 


\section{SONY}

make.believe

ICD-SX712

The ICD-SX712 is loaded with features ideal for frequent and professional users. The unique 2-position microphone offers powerful recording capability while the Sony exclusive Intelligent Noise Cut ${ }^{\mathrm{TM}}$ mode dramatically reduces background noise from recordings for supreme clarity during playback. Add in CD quality recording with Linear PCM recording mode, manual gain control and expandable memory for the ultimate in digital recording capability and convenience.

\section{2-POSITION MIC}

The 2-position microphone on the SX712 offers the most powerful recording capability in the Sony ICD lineup. The dual microphone array can be set in two positions: Dictation / Meetings \& Conferences.

\section{RECORDING QUALITY}

The SX712 provides flexibility in audio recording and playback. Audio can be recorded in CD quality with Linear PCM recording mode or in commonly used MP3 format.

\section{INTELLIGENT NOISE CUT ${ }^{\mathrm{TM}}$ MODE}

A unique and innovative technology from Sony, Intelligent Noise Cut ${ }^{\mathrm{TM}}$ mode significantly reduces ambient noise from recordings so that playback can be heard more clearly.

\section{MANUAL / AUTOMATIC RECORD LEVEL}

Ability to enable the recorder to automatically adjust the recording level or manually adjust it. The manual setting is especially useful for musicians.

\section{MEMORY - EXPANDABLE}

A Memory Card slot offers access to additional recording time using optional flash memory media that can be removed and stored or used to transfer recordings to a computer.

\section{A-B REPEAT}

Mark the beginning and ending of a particular recorded passage and the recording will be played over and over again- great for language learning.

\section{ADD RECORDING}

Enables the user to press the RECORD button during playback and automatically add additional recording at the end without affecting the original recording.

\section{ALARM}

Set a future date and time and the recording will automatically be played.

BACKLIT DISPLAY

Convenient when using the unit in a low light environment.

\section{CALENDAR VIEW}

Displays a calendar that indicates the dates when recordings were made and enables searching of a specific date. CROSS MEMORY RECORDING

Enables the recorder to automatically extend the recording from the built-in flash memory to an optional micro SD card without interruption.

\section{DICTATION CORRECTION}

Enables the user to press the RECORD button during playback and overwrite the recording correcting the original recording.

DPC

Digital Pitch Control- enables the recording to be played at a faster or slower rate without changing the pitch.

\section{DPC KEY CONTROL}

A visual feature on the LCD display allowing the user to adjust the playback speed for music or voice recording playback.

DRAGON COMPATIBLE / CERTIFIED 


\section{SONY}

make.believe

The recording quality of the unit has been optimized and validated by Nuance to work with the Voice to Print Dragon NaturallySpeaking ${ }^{\circledR}$ software.

\section{EARPHONE JACK}

A listening device can be plugged into the recorder.

EASY SEARCH

Rewinds or Fast Forwards in short increments making it easier to locate a segment in a long recording for review. GRAPHIC EQUALIZER

Enables the user to fine tune the unit for the best sounding music playback.

HI / LO MICROPHONE SENSITIVITY

Set the recorder for dictation and notes or for conferences and lectures.

MIC JACK

Provides the ability to use an external microphone.

MULTI LANGUAGE MENU

Enables the menu language to be displayed in English, French or Spanish. MUSIC FOLDER AND PODCAST FOLDER

Creates new Music and Podcast tab after "Ripping" a CD and downloading to the unit. PERSONALIZED FOLDER NAMES ( $w / a$ PC)

Allows the folder names to be personalized such as NOTES, HISTORY CLASS, SCHEDULE etc.

PLAYBACK

For playback, the SX712 supports MP3/WMA/AAC files.

PRE-RECORD FUNCTION

A feature desirable for individuals who record interviews such as press people which buffers the recording to make sure the initial words spoken by the subject are not missed.

RECORD LEVEL METER

In the manual record level mode it visually displays the RECORDING LEVEL of the left and right channels. RECORD/PAUSE

Provides the ability to pause the recording process, gather your thoughts and then continue recording in one easy step.

S MIC SYSTEM

A newly developed recording system that results in high sensitivity and less noise through dedicated tuning of each different model type.

\section{SCENE SELECT}

An easy to use system that allows the user to select how they are going to use the recorder, and the device automatically adjusts the unit to the optimal settings: notes, lectures, music etc.

\section{SOUND ORGANIZER SOFTWARE BUNDLE}

Software bundle that allows editing, viewing track marks, CD/DVD burning, playback with Intelligent Noise Cut ${ }^{\mathrm{TM}}$ and other useful features.

\section{STEREO MICS}

The recorder has stereo microphones eliminating the need to use an external microphone.

SUPPLIED STAND AND CARRYING POUCH

Convenient accessories supplied with the unit to enhance the user experience.

TRACK MARK

Place up to 98 track marks for each recorded file which can be "jumped" to for playback.

USB CHARGE 


\section{SONY}

\section{make.believe}

Ability to charge rechargeable batteries in the recorder via USB connection. Rechargeable batteries included with ICD-SX712D. Sold separately for ICD-SX712.

\section{USB PC \& MAC ${ }^{\circ}$ COMPATIBLE}

Works with Windows ${ }^{\circ}$ as well as Macintosh ${ }^{\circ}$ computers.

VOR

Voice Operated Recording starts and stops the recording when sound of a sufficient level is picked up by the microphone. This feature saves valuable recording time by not recording periods when there is little or no sound. WIDE DYNAMIC RANGE RECORDING

Designed for extremely high quality recordings with a wide frequency response.

Specifications

\begin{tabular}{|c|c|}
\hline \multicolumn{2}{|l|}{ Audio } \\
\hline Format(s) Supported & $\begin{array}{l}\text { MP3 files: } \\
\text { Bit Rate: } 8 \text { kbps - } 320 \text { kbps, VBR } \\
\text { Sampling Frequencies: 16/22.05/24/32/44.1/48 kHz } \\
\text { File Extension: .mp3 } \\
\text { br } \\
\text { WMA files: } \\
\text { Bit Rate: } 32 \text { kbps - } 192 \text { kbps, VBR } \\
\text { Sampling Frequencies: } 44.1 \text { kHz } \\
\text { File Extension: .wma } \\
\text { br } \\
\text { AAC-LC files*: } \\
\text { Bit Rate: } 16 \text { kbps - } 320 \text { kbps, VBR } \\
\text { Sampling Frequencies: } 11.025 / 12 / 16 / 22.05 / 24 / 32 / 44.1 / 48 \mathrm{kHz} \\
\text { File Extension: .m4a } \\
\text { * Copyright-protected files playback not supported } \\
\text { br } \\
\text { PCM files: } \\
\text { Bit Rate: } 16 \text { bit } \\
\text { Sampling Frequencies: } 22.05 / 44.1 \mathrm{kHz} \\
\text { File Extension: .wav }\end{array}$ \\
\hline Frequency Range & $\begin{array}{l}\text { LPCM 44/16: } 40-20,000 \mathrm{~Hz} \\
\text { MP3 320k: } 40-16,000 \mathrm{~Hz} \\
\text { MP3 192k: } 40-16,000 \mathrm{~Hz} \\
\text { MP3 128k: } 40-16,000 \mathrm{~Hz} \\
\text { MP3 48k (Mono): } 40-14,000 \mathrm{~Hz} \\
\text { MP3 8k (Mono): } 50-2,000 \mathrm{~Hz}\end{array}$ \\
\hline Microphone & Built-in, Stereo microphone with rotating capsules \\
\hline Recording System & Digital Stereo, Monaural Recording \\
\hline \multicolumn{2}{|l|}{ Audio Features } \\
\hline Microphone Sensitivity Feature & Yes (SENS Settings) \\
\hline Recording Features & 6 Modes: Linear PCM / MP3 320kbps / MP3 192kbps / MP3 128kbps / MP3 48kbps (Mono) / MP3 8kbps (Mono) \\
\hline \multicolumn{2}{|l|}{ Convenience Features } \\
\hline Erase/Protect & Yes \\
\hline Message Folder(s) & Yes (5 Folders) \\
\hline On-Screen Clock & Yes, with Calendar \\
\hline Remote Control & No \\
\hline Safety Lock & Yes (Hold Function) \\
\hline
\end{tabular}




\section{SONY}

\section{make.believe}

\begin{tabular}{|c|c|}
\hline Search Features & Cue/Review, Easy Search, Calendar Search, Track Mark \\
\hline Voice E-Mail Function & Yes \\
\hline \multicolumn{2}{|l|}{ General } \\
\hline \multirow{2}{*}{ Recording and Playback Times } & $\begin{array}{l}\text { brMP3-44.1 kHz/192k: } 23 \mathrm{H} 35 \mathrm{MbrMP} 3-44.1 \mathrm{kHz} / 128 \mathrm{k}: 35 \mathrm{H} 25 \mathrm{MbrMP} 3-44.1 \mathrm{kHz} / 48 \mathrm{k}: 94 \mathrm{H} 40 \mathrm{MbrSTHQ}-44.1 \mathrm{kHz} / 128 \mathrm{k}: 34 \mathrm{H} \\
55 \mathrm{MbrLPCM}-44.1 \mathrm{kHz} / 16 \mathrm{bit} / \mathrm{WAV}: 3 \mathrm{H} 10 \mathrm{Mbr}\end{array}$ \\
\hline & $\begin{array}{l}\text { LPCM-22/16 bit/WAV: } 6 \mathrm{H} \text { 25MbrST-44.1kHz/48k: 92H 15MbrSTLP-16kHz/24k: 177H 40MbrSP-16kHz/16k: 281H 20MbrLP- } \\
8 \mathrm{kHz} / 6 \mathrm{k}: 750 \mathrm{H} 15 \mathrm{M}^{2}\end{array}$ \\
\hline Recording Media & Built-In Flash Memory 2GB and Memory Card Expansion Slot (microSD/M2) \\
\hline Type of Use & Ultra Portable Voice Recorder \\
\hline \multicolumn{2}{|l|}{ Hardware } \\
\hline Display & Full Function LCD Display with LED operation indicator \\
\hline \multicolumn{2}{|l|}{ Inputs and Outputs } \\
\hline Headphone Output(s) & 1 (Mini jack) \\
\hline Microphone Input & 1 (Mini jack stereo) \\
\hline USB Port(s) & 1 (Mini-B jack), High-Speed USB Compatible \\
\hline \multicolumn{2}{|l|}{ Memory } \\
\hline Memory Size & $\begin{array}{l}2 \text { GB (approx. } 1.9 \mathrm{~GB}=2,147,483,648 \text { bytes) } \\
\text { Available: } 1.8 \mathrm{~GB}(1,932,735,283 \text { bytes) }\end{array}$ \\
\hline \multicolumn{2}{|l|}{ Power } \\
\hline Battery Type & "AAA" x 2 \\
\hline Output Power & $150 \mathrm{~mW}$ \\
\hline Power Requirements & $\begin{array}{l}\text { Two LR03 (size AAA) alkaline batteries: } 3.0 \text { V DC } \\
\text { Two NH-AAA rechargeable batteries: } 2.4 \text { V DC }\end{array}$ \\
\hline \multicolumn{2}{|l|}{ Software } \\
\hline Operating System Compatibility & 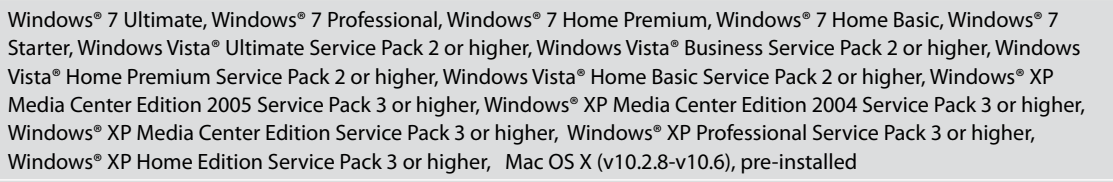 \\
\hline Supplied Software & Application Software, Sound Organizer (CD-ROM) \\
\hline \multicolumn{2}{|l|}{ Speaker } \\
\hline Speaker Dimensions & Approx. $21 / 32$ in. $(16 \mathrm{~mm})$ diameter \\
\hline \multicolumn{2}{|l|}{ Weights and Measurements } \\
\hline Dimensions (Approx.) & $11 / 4 \times 53 / 8 \times 5 / 8$ in. \\
\hline Weight (Approx.) & $23 / 8$ oz. (68 g) excluding batteries \\
\hline \multicolumn{2}{|l|}{ Accessories } \\
\hline Supplied Accessories & $\begin{array}{l}\text { IC Recorder (1) } \\
\text { LR03 (size AAA) alkaline batteries ( } 2 \text { ) } \\
\text { Stereo headphones (1) } \\
\text { Wind Screen (1) } \\
\text { USB connecting cable (1) } \\
\text { Application Software, Sound Organizer (CD-ROM) } \\
\text { Cradle* (1) } \\
\text { Soft carrying pouch (1) } \\
\text { Battery case* (1) } \\
\text { Stand (1) } \\
\text { Operating Instructions } \\
\text { * The cradle, the battery case, and some of the software are supplied with some models in some regions only. }\end{array}$ \\
\hline
\end{tabular}




\section{SONY}

make.believe

Optional Accessories

Memory Stick Micro ${ }^{\text {TM }}\left(M^{2}{ }^{\text {TM }}\right)$ : MS-A16GU2, MS-A8GU2, MS-A4GU2, MS-A2GU2

microSD/microSDH: SR-8A4, SR-4A4, SR-2A1

Active Speaker: SRS-M50

Optional Accessories

Electret Condenser Microphone: ECM-CS10, ECM-TL1

Audio Connecting Cable: RK-G136, RK-G139

Rechargeable battery NH-AAA-B2KN

USB AC adaptor AC-U501AD (U.S.A. and Canada only)

1. Copy protected content not supported.

2. $1 \mathrm{~GB}=1$ billion bytes, a portion of which is used for data management functions.

O 2011 Sony Electronics Inc. All rights reserved. Reproduction in whole or in part without written permission is prohibited. Sony and Intelligent Noise Cut are trademarks of Sony. Windows is a registered trademark of Microsoft Corporation in the United States and/or other countries. Macintosh and Mac are registered trademarks of Apple Inc. Dragon NaturallySpeaking are registered trademarks of Nuance Communications, Inc. Al other trademarks are trademarks of their respective owners. Features and specifications are subject to change without notice.

Updated: June 28,201 
Appendix F

PSD Analysis for Taxiing and Cruise 


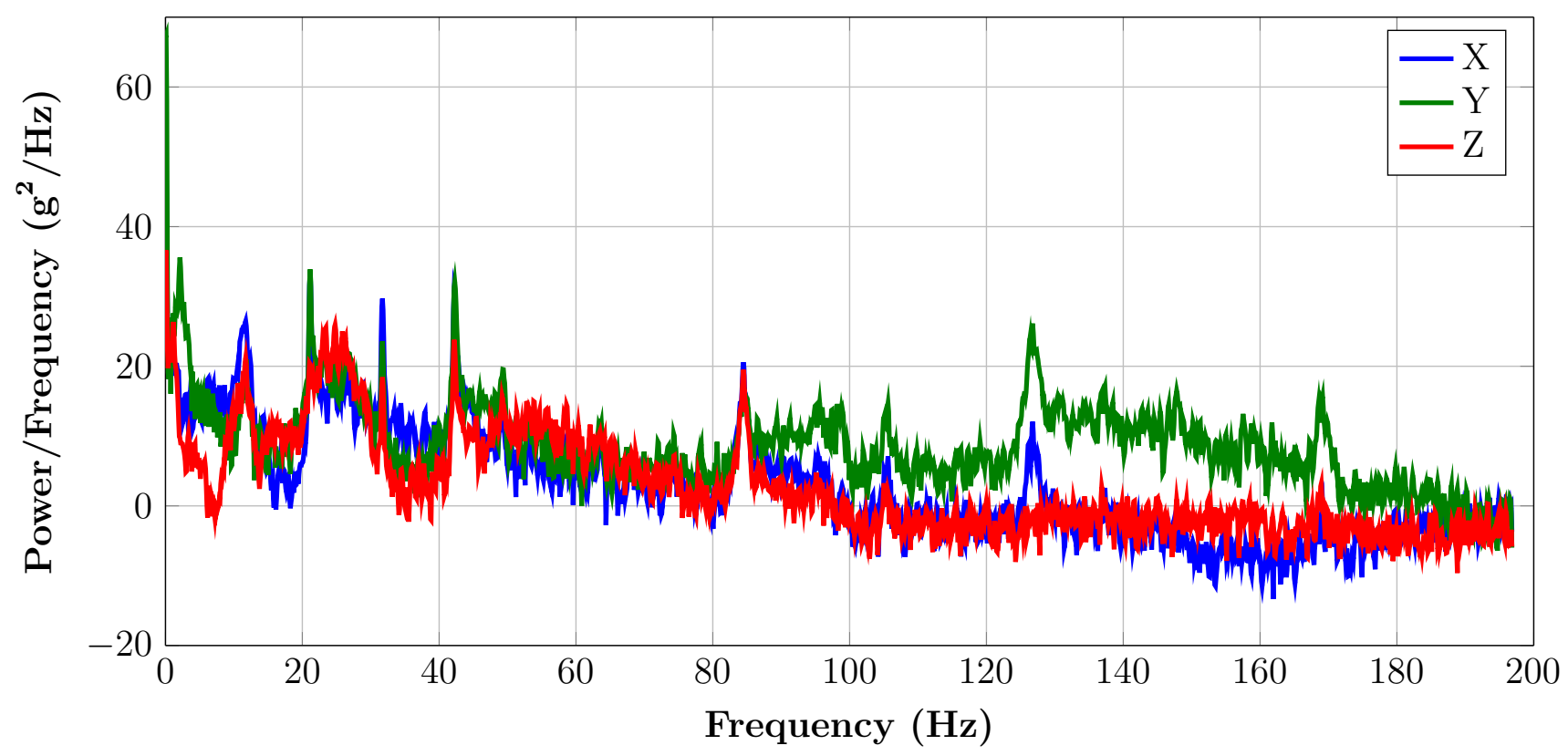

Figure F.1: PSD analysis of the vibration of the aircraft taxiing at 1000 RPM.

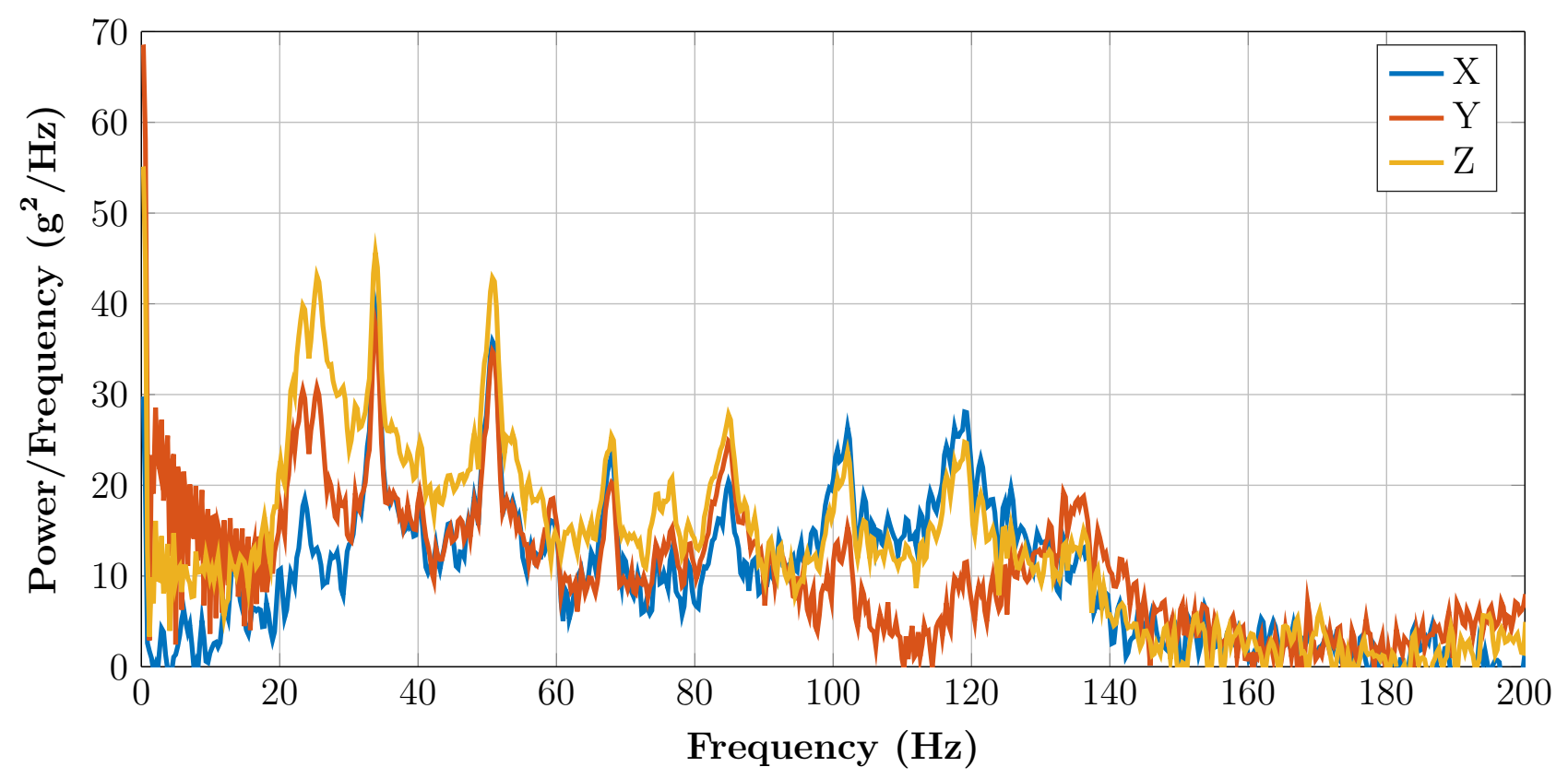

Figure F.2: PSD analysis of the vibration of the simulator taxiing at 1000 RPM. 


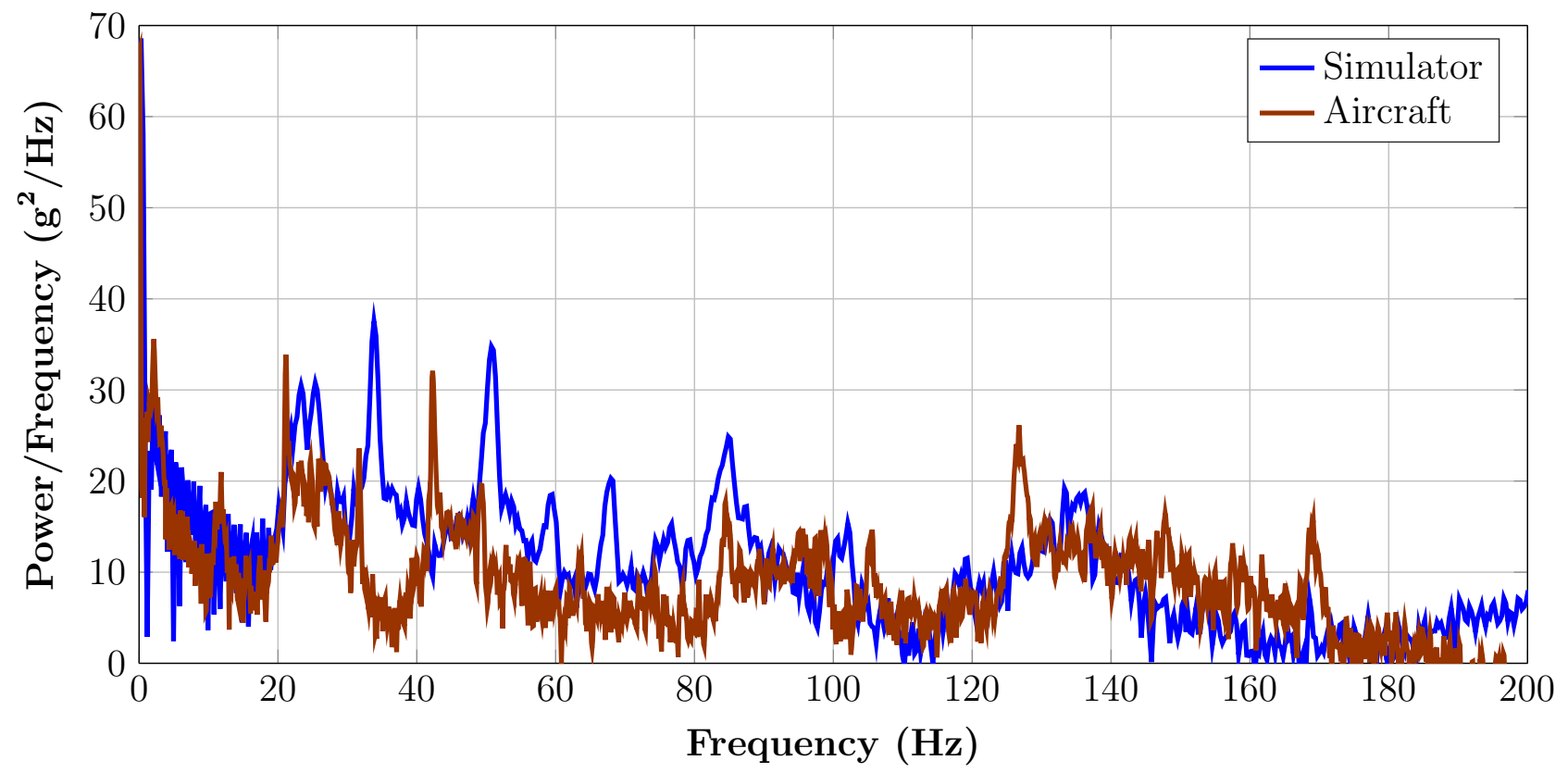

Figure F.3: PSD analysis of the vibration of the simulator and aircraft in the Y direction taxiing at 1000 RPM.

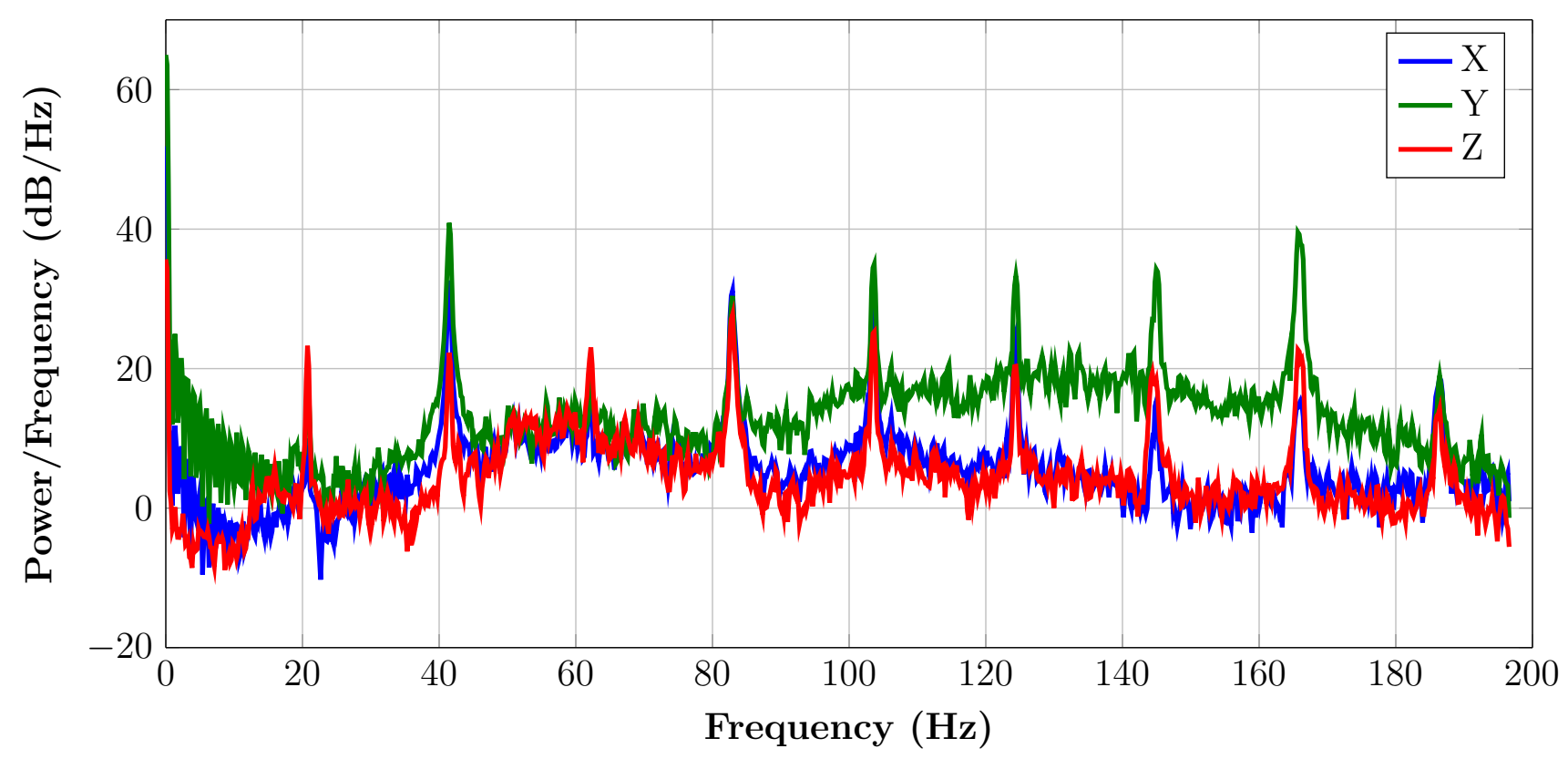

Figure F.4: PSD analysis of the vibration of the aircraft cruising at 2400 RPM. 


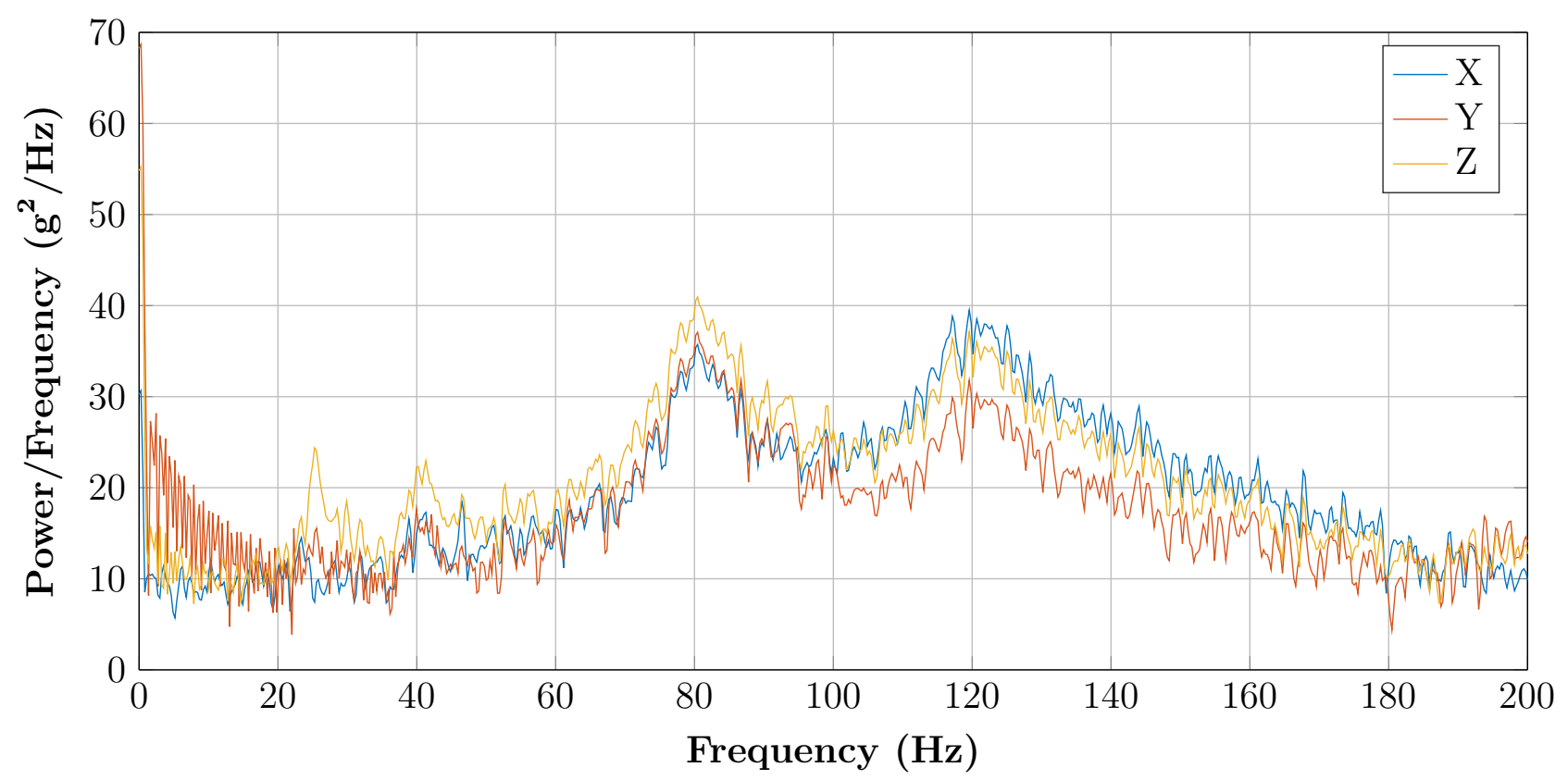

Figure F.5: PSD analysis of the vibration of the aircraft cruising at 2400 RPM.

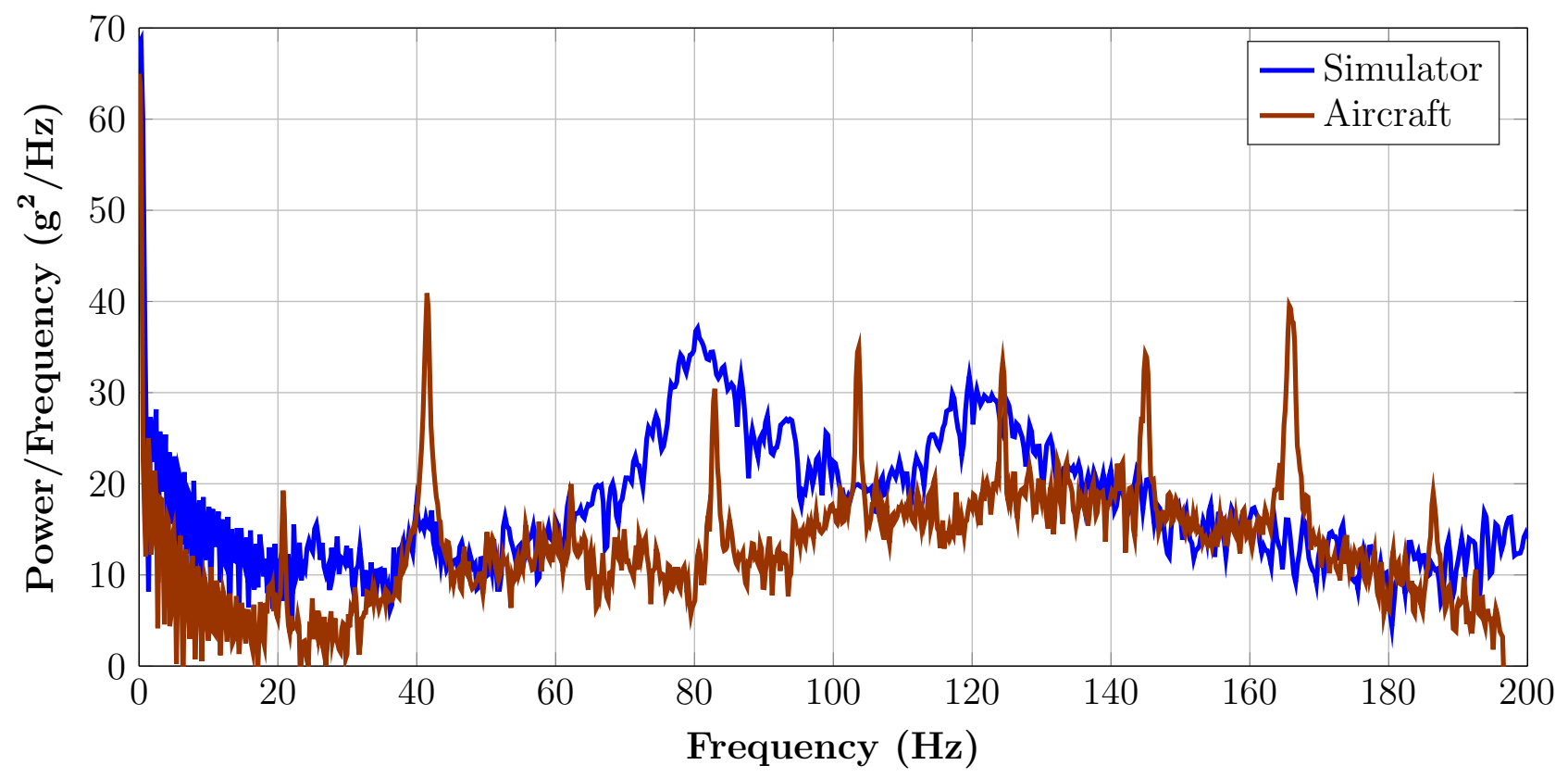

Figure F.6: PSD analysis of the vibration of the simulator and the aircraft in the Y direction cruising at 2400 RPM. 


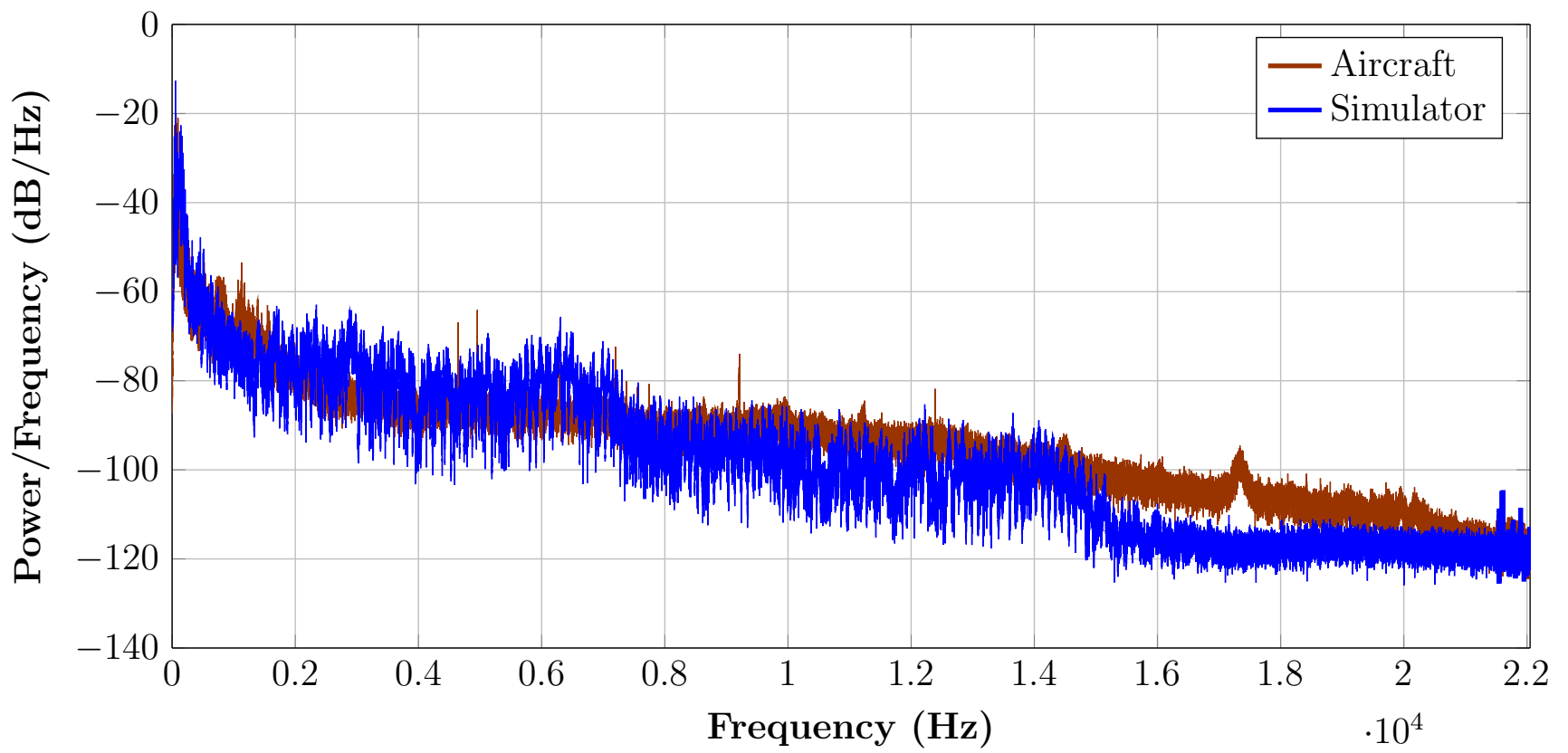

Figure F.7: Full-range PSD analysis comparing the sound of the simulator and the aircraft taxiing at 1000 RPM.

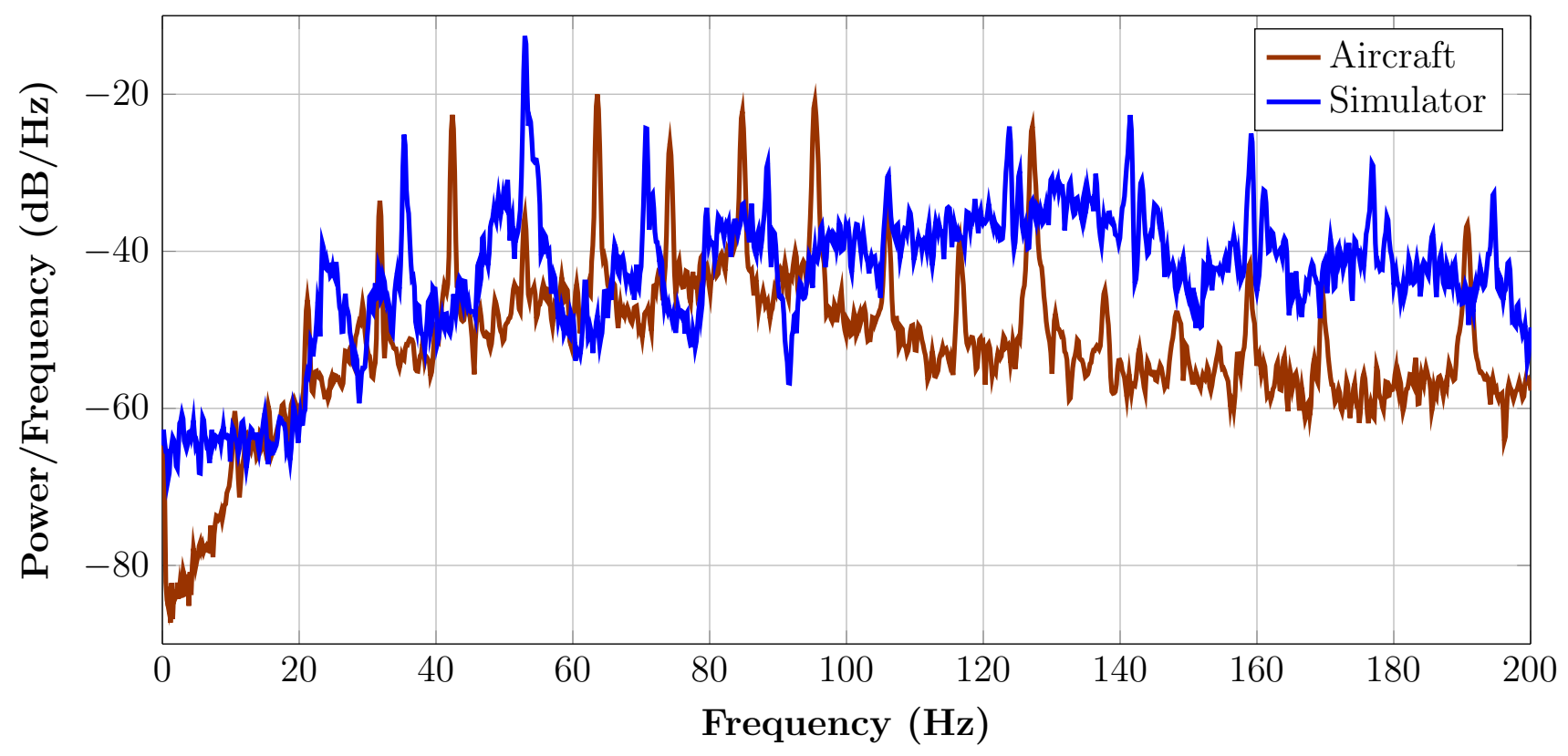

Figure F.8: Low-frequency range PSD analysis comparing the sound of the simulator and the aircraft taxiing at 1000 RPM. 


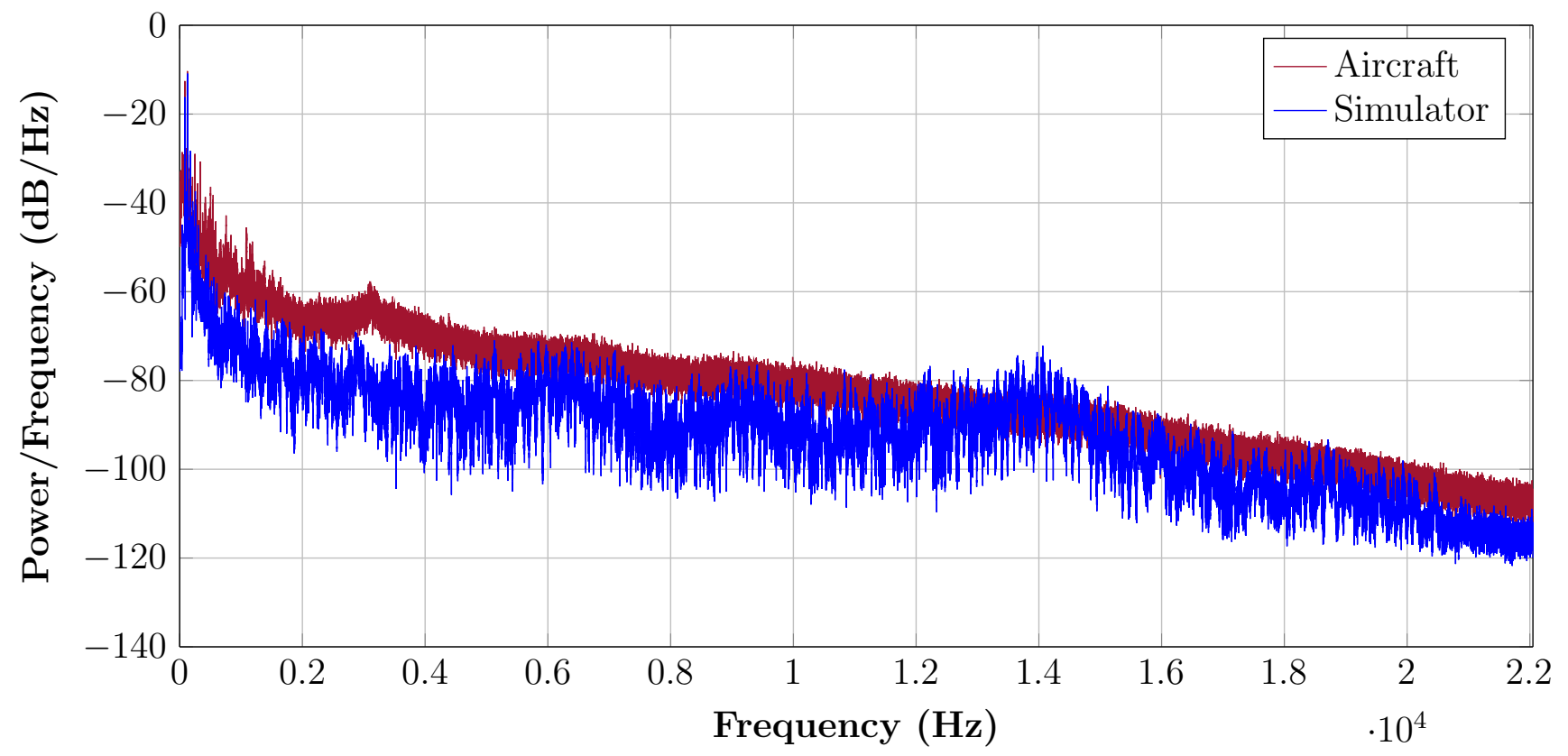

Figure F.9: Full-range PSD analysis comparing the sound of the simulator and the aircraft cruising at 2400 RPM.

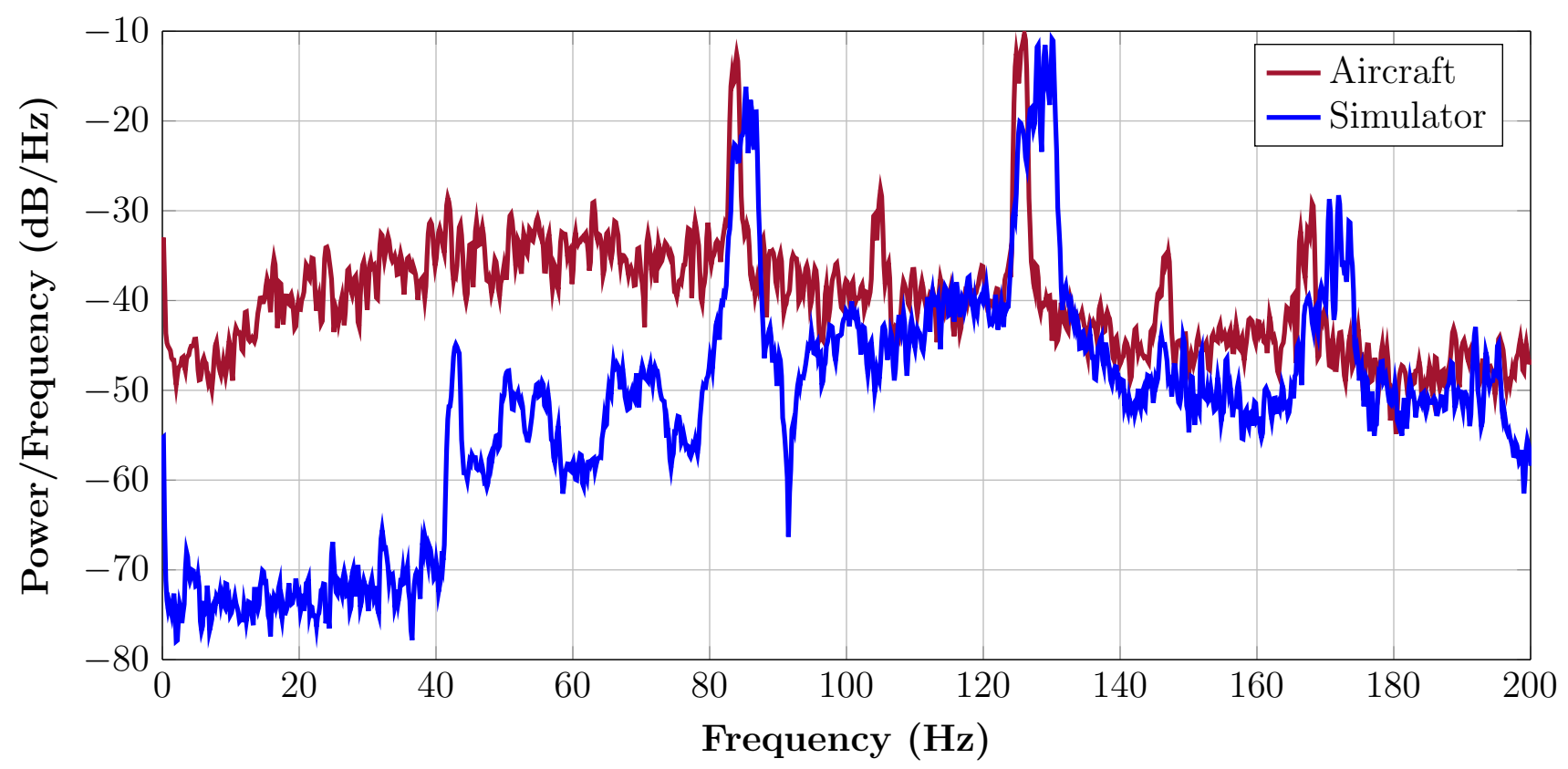

Figure F.10: Low-frequency range PSD analysis comparing the sound of the simulator and the aircraft cruising at 2400 RPM. 


\section{Appendix G}

\section{Matlab Codes for PSD Analysis}




\section{G.1 Vibration Analysis Matlab Code}

${ }_{1} \%$ Hooman Rajaee Code for the CURVS project

${ }_{2} \%$ In order to plot the data and do PSD analysis using Welch method,

$3 \%$ simply import the desired set of data from the CSV files of the

${ }_{4} \%$ accelerometer. Before doing so make sure that the Time, Ax, Ay, Az

${ }_{5} \%$ columns are renamed to Time, Ax, Ay, Az

6

7

8 SimTime $=$ SimTime - SimTime $(1) ; \quad \%$ Resets the time to zero

${ }_{9} \operatorname{SimAxx}=\operatorname{SimAx} / 2048 ; \quad \%$ Acceleration data to $\mathrm{G}$ forces

${ }_{10} \quad$ SimAyy $=$ SimAy $/ 2048$;

${ }_{11} \quad \operatorname{SimAzz}=\operatorname{SimAz} / 2048$;

12

${ }_{13} \%$ Plot all the Time-Domain data together in one shot

14 figure (6)

15 plot (SimTime, SimAxx, SimTime, SimAyy, SimTime, SimAzz)

16 ylabel ('Acceleration (g)')

17 xlabel ('Time')

18 grid on

$19 \operatorname{axis}\left(\left[\begin{array}{llll}0 & 10 & -0.5 & 1.5\end{array}\right]\right)$

${ }_{20}$ legend ('X', 'Y' , 'Z') 


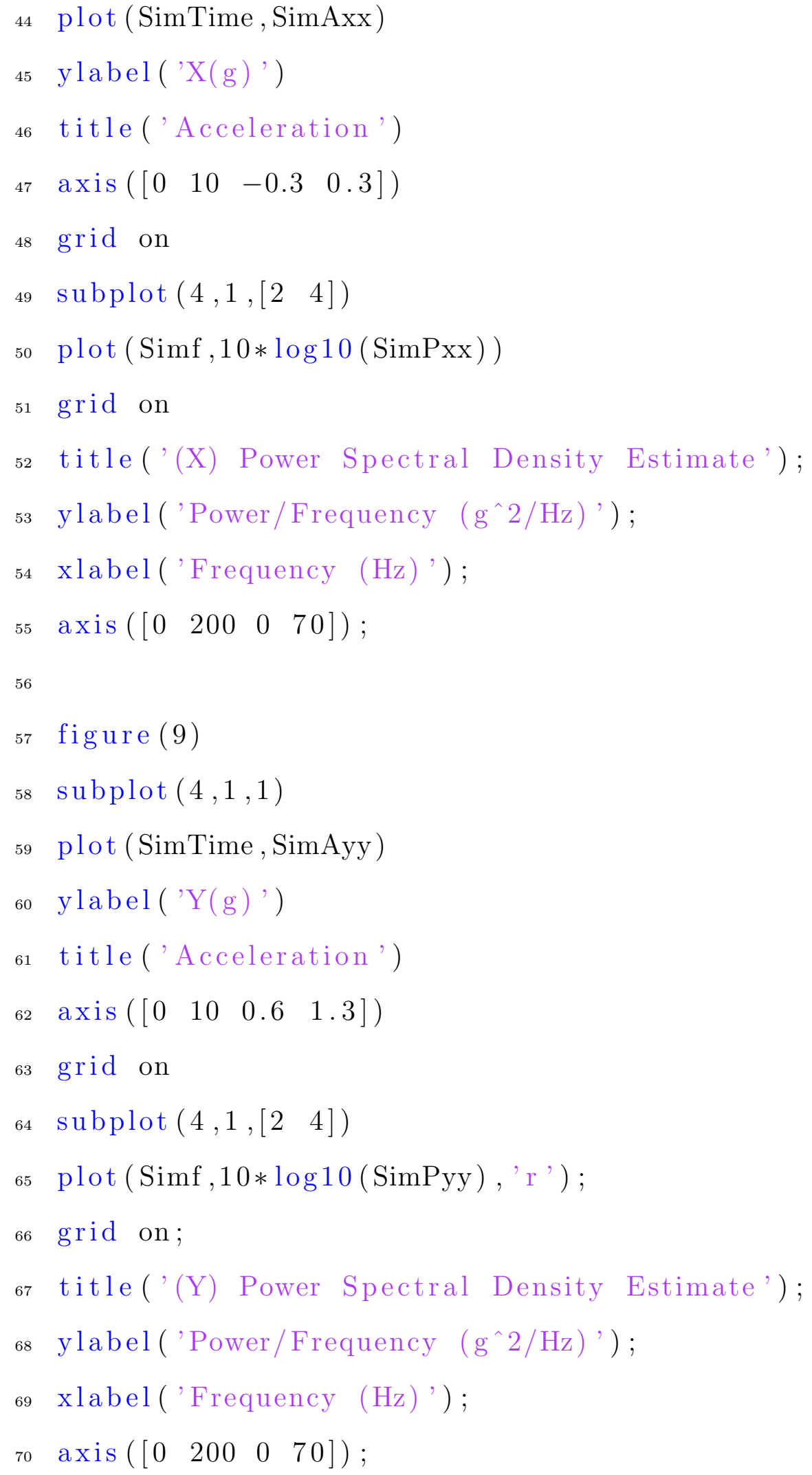


72 figure (10)

73 subplot $(2,1,1)$

74 plot (SimTime, SimAzz)

75 xlabel ('Time (sec)')

76 ylabel('Z(g)')

$77 \operatorname{axis}\left(\left[\begin{array}{llll}0 & 10 & -1 & 1.2\end{array}\right]\right)$

78 grid on

79 subplot $(2,1,2)$

$80 \operatorname{plot}\left(\operatorname{Simf}, 10 * \log 10(\operatorname{SimPzz}),{ }^{\prime} r{ }^{\prime}\right)$;

81 grid on;

82 title('(Z) Power Spectral Density Estimate');

83 ylabel ('Power/Frequency ( g $\left.^{\wedge} 2 / \mathrm{Hz}\right)$ ') ;

84 xlabel ('Frequency (Hz) ') ;

$\left.85 x \lim \left(\left[\begin{array}{ll}0 & 200\end{array}\right]\right)\right\}$

\section{G.2 Sound Analysis Matlab Code}

${ }_{1}[\mathrm{x}, \mathrm{fs}]=\mathrm{wavread}($ 'track.wav') $\quad \%[\mathrm{y}, \mathrm{Fs}]=\operatorname{wavread}($ filename $)$ returns the sample rate (Fs) in Hertz used to encode the data in the file.

${ }_{2} \mathrm{~N}=\operatorname{length}(\mathrm{x})$;

$3 \mathrm{t}=(0: \mathrm{N}-1) / \mathrm{fs}$;

$4 \%$ plotting of the waveform

$5 \quad$ figure (1)

${ }_{6} \operatorname{plot}\left(\mathrm{t}, \mathrm{x},{ }^{\prime} \mathrm{r}\right.$ ')

$7 x \lim ([0 \max (\mathrm{t})])$ 
$8 \operatorname{ylim}([-1.1 * \max (\operatorname{abs}(\mathrm{x})) \quad 1.1 * \max (\operatorname{abs}(\mathrm{x}))])$

9 grid on

10 set(gca, 'FontName', 'Times New Roman', 'FontSize', 14)

11 xlabel ('Time, s')

12 ylabel('Normalized amplitude')

13 title('The signal in the time domain') linebreak

14

${ }_{15}[\mathrm{Px}, \mathrm{f}]=\operatorname{pwelch}(\mathrm{x},[],[],[], \mathrm{fs}) ;: \%$ Calculate PSD using Welch

$16 \operatorname{plot}(\mathrm{f}, 10 * \log 10(\mathrm{Px}))$

17 grid on

18 title('Power Spectral Density Estimate')

19 xlabel ('Frequency (Hz)')

20 ylabel ('Power/Frequency (dB/Hz)')\} 


\section{Appendix $\mathbf{H}$ \\ Participants Information and the Questionnaire}


Table H.1: List of qualitative test participants.

\begin{tabular}{|c|c|c|c|c|c|c|c|}
\hline Number & Name & Age & Gender & $\begin{array}{c}\text { Pilot } \\
\text { Licence }\end{array}$ & $\begin{array}{c}\text { Hours } \\
\text { Flown }\end{array}$ & $\begin{array}{c}\text { C172 } \\
\text { Hours }\end{array}$ & $\begin{array}{c}\text { Simulator } \\
\text { Hours }\end{array}$ \\
\hline Participant 1 & Veronika Bujaki & 20 & F & PPL & 110 & 50 & 11 \\
\hline Participant 2 & Andrew Lumley & 20 & M & PPL & 30 & 20 & 2 \\
\hline Participant 3 & Jason Etele & 40 & M & PPL & $100+$ & $50+$ & N/A \\
\hline Participant 4 & Chris Hobbs & 66 & M & CPL & 2494 & 2000 & 60 \\
\hline Participant 5 & Andre Durocher & 60 & M & ATPL & 7800 & 100 & 100 \\
\hline Participant 6 & Brent Cameron & 27 & M & Student Pilot & 40 & 1 & N/A \\
\hline Participant 7 & Matthew Parson & 47 & M & $\begin{array}{c}\text { Canadian Forces } \\
\text { Test Pilot (CPL) }\end{array}$ & 4000 & 4 & 700 \\
\hline Participant 8 & Suzanne Swaine & 27 & F & PPL & 70 & 0 & 5 \\
\hline
\end{tabular}




\section{CURVS Feedback Test}

The objective of this test is to collect qualitative feedback from pilots who have experience in flying a Cessna 172 and as well as a Cessna 172 FSTD. The test is designed to involve two phases. Phase I is to test the visual system, sound system, and vibratory system of the CURVS FSTD by performing takeoff, landing and circuit pattern manoeuvers in a VFR flight environment intended for ab-initio training. Phase $I I$ is to test the instruments of the simulator and their performance while practicing in IFR conditions. The questionnaire is made of multiple choice questions followed by questions that require further explanation. The questionnaire is adapted from a NASA simulator test technical paper ${ }^{1}$.

\section{Phase I: VFR flying}

Personal Data

1. Name:

2. Age:

3. Gender:

4. Occupation:

5. Contact Information:

Flying Data

1. Highest pilot license:

2. Number of hours flown:

3. Types of aircraft flown:

4. Number of hours flown in a C172:

5. Number of hours flown during the last 2 months in a C172:

6. The last time flew a $\mathrm{C} 172$ before the test:

\section{Simulator Data}

1. Approximately how much total simulator time do you have in your logbook?

2. How many hours do you have in Cessna 172 simulator? Please explain your experience.

\footnotetext{
${ }^{1}$ Benton L. Parris and Anthony M. Cook, Effects of Visual and Motion Simulation Cueing Systems During Takeoffs with Engine Failure, published by NASA, Dec 1978
} 


\section{Visual Cueing}

1. Rate your general experience with the CURVS visual system in comparison to the actual Cessna 172.
- Excellent
- Good
- $\underline{\text { Fair }}$
- Poor
- Very Poor

Comments:

2. Rate your general experience with the CURVS visual system in comparison to other simulators (if applicable).
- Excellent
- Good
- Fair
- Poor
- Very Poor

Comments: 
3. Rate the field-of-view (FOV) of the CURVS visual system.
○ Excellent
○ Good
O Fair
○ Poor
- Very Poor

4. Was the lateral FOV sufficient for practicing all the manoeuvers in ab-inito training?

Yes $\quad$ No Please explain:

5. Did the extra FOV for over-shoulder view on the pilot side provide a more realistic experience for a left-hand circuit?

Yes No Please explain:

6. Was the vertical field of view sufficient?

Yes No Please explain:

7. Was the general $2 / 3$ sky and $1 / 3$ ground rule while flying at cruise satisfied?

Yes No Please explain:

Comments: 
8. How do you rate the screen resolution?
- Excellent
- Good
- Fair
- Poor
- Very Poor

9. Were you able to see the individual pixels (screen door effect)?

Yes No Please explain:

10. Were you able to recognize different objects while flying (For instance, roads, trees, runways, buildings, etc.)?

Yes No Please explain:

11. Were you able to recognize the end of the runway in over-shoulder view at the end of the downwind leg of the circuit?

Yes No Please explain:

12. How did the screen resolution impact your approaches, especially on final? Where you able to recognize runway markings, numbers, PAPI lights, etc.?

Yes No Please explain:

Comments: 
13. How do you rate the frame rate (FPS)?
- Excellent
○ Good
○ $\underline{\text { Fair }}$
- Poor
- Very Poor

14. In all the stages of your flight did you feel the smoothness of the simulation? How close was it to the real aircraft experience?

Yes No Please explain:

15. Did you experience any lag or jitteriness of the visual system? If yes, how did it impact your flight experience and your response to controls?

Yes No Please explain:

16. During your test did you experience any lag, latency, or mismatch between the images of the projectors? Please provide some feedback.

Yes No Please explain:

17. During approaches and landings, when you were closer to the aerodrome with more dense 3D objects did you experience any drops in frame rates? Did it affect your decisions in landing?

Yes No Please explain:

Comments: 


\section{Sound Cueing}

18. How do you rate the sound system?
- Excellent
- Good
○ Fair
- Poor
- Very Poor

19. Did the engine noise resemble actual C172 aircraft noise?

Yes No Please explain:

20. Was the loudness level of the engine noise sufficient?

Yes No Please explain:

21. Do you think there was a realistic relationship between different engine RPMs and the pitch of the noise?

Yes No Please explain:

22. Did you notice any unrealistic sound from any sources such as skidding while touching down, flaps, etc.?

Yes No Please explain:

Comments: 


\section{Vibratory Cueing}

23. How do you rate the vibratory cueing in general?
- Excellent
- Good
- Fair
- Poor
- Very Poor

24. Do you think it had a significant impact on the realism of the simulation? Please rate it on a scale of 1 to 4 .

- 1 - No positive impact at all

○ 2 - I could feel it but its existence doesn't affect the fidelity

○ 3 - It played a positive role in the fidelity of the simulation

○ 4 - It remarkably added to the realism

25. In which part of the flight did the vibrations seem most noticeable?

26. Did the vibration help you to recognize the air-to-ground and ground-to-air transition during touchdowns and rotations?

Yes No Please explain:

27. Do you think the intensity of the vibration was realistic? Should it be lower or higher?

Yes No Please explain:

Comments: 